NUREG/CR-6021

CNWRA 92-011

\title{
A Literature Review of Coupled Thermal-Hydrologic-Mechanical- Chemical Processes Pertinent to the Proposed High-Level \\ Nuclear Waste Repository at Yucca Mountain
}

Manuscript Completed: February 1993

Date Published: July 1993

Prepared by

R. D. Manteufel, M. P. Ahola, D. R. Turner, A. H. Chowdhury

Center for Nuclear Waste Regulatory Analyses

Southwest Research Institute

6220 Culebra Road

San Antonio, TX 78238-5166

Prepared for

Division of High-Level Waste Management

Office of Nuclear Material Safety and Safeguards

U.S. Nuclear Regulatory Commission

Washington, DC 20555-0001

NRC FIN D1035 


\section{DISCLAIMER}

This report was prepared as an account of work sponsored by an agency of the United States Government. Neither the United States Government nor any agency thereof, nor any of their employees, make any warranty, express or implied, or assumes any legal liability or responsibility for the accuracy, completeness, or usefuiness of any information, apparatus, product, or process disclosed, or represents that its use would not infringe privately owned rights. Reference herein to any specific commercial product, process, or service by trade name, trademark, manufacturer, or otherwise does not necessarily constitute or imply its endorsement, recommendation, or favoring by the United States Government or any agency thereof. The views and opinions of authors expressed herein do not necessarily state or reflect those of the United States Government or any agency thereof. 


\section{DISCLAIMER}

Portions of this document may be illegible in electronic image products. Images are produced from the best available original document. 


\begin{abstract}
A literature review has been conducted to determine the state of knowledge available in the modeling of coupled thermal $(\mathrm{T})$, hydrologic $(\mathrm{H})$, mechanical $(\mathrm{M})$, and chemical $(\mathrm{C})$ processes relevant to the design and/or performance of the proposed high-level waste (HLW) repository at Yucca Mountain, Nevada. The review focuses on identifying coupling mechanisms between individual processes and assessing their importance (i.e., if the coupling is either important, potentially important, or negligible). The significance of considering THMC-coupled processes lies in whether or not the processes impact the design and/or performance objectives of the repository.

A review, such as reported here, is useful in identifying which coupled effects will be important, hence which coupled effects will need to be investigated by the U.S. Nuclear Regulatory Commission in order to assess the assumptions, data, analyses, and conclusions in the design and performance assessment of a geologic repository. Although this work stems from regulatory interest in the design of the geologic repository, it should be emphasized that the repository design implicitly considers all of the repository performance objectives, including those associated with the time after permanent closure. The scope of this review is considered beyond previous assessments in that it attempts (with the current state-ofknowledge) to determine which couplings are important, and identify which computer codes are currently available to model coupled processes.
\end{abstract}




\section{CONTENTS}

Section

Page

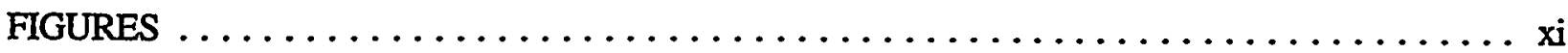

TABLES $\ldots \ldots \ldots \ldots \ldots \ldots \ldots \ldots \ldots \ldots \ldots \ldots \ldots \ldots \ldots \ldots \ldots \ldots \ldots \ldots \ldots \ldots \ldots$

NOMENCLATURE $\ldots \ldots \ldots \ldots \ldots \ldots \ldots \ldots \ldots \ldots \ldots \ldots \ldots \ldots \ldots$ xvii

ACKNOWLEDGEMENTS $\ldots \ldots \ldots \ldots \ldots \ldots \ldots \ldots \ldots \ldots \ldots \ldots \ldots \ldots \ldots \ldots \ldots$ xxiii

EXECUTIVE SUMMARY $\ldots \ldots \ldots \ldots \ldots \ldots \ldots \ldots \ldots \ldots \ldots \ldots \ldots \ldots \ldots \ldots$

1 INTRODUCTION $\ldots \ldots \ldots \ldots \ldots \ldots \ldots \ldots \ldots \ldots \ldots \ldots \ldots \ldots \ldots \ldots \ldots$

1.1 STATE OF COUPLING $\ldots \ldots \ldots \ldots \ldots \ldots \ldots \ldots \ldots \ldots \ldots \ldots \ldots \ldots \ldots \ldots$

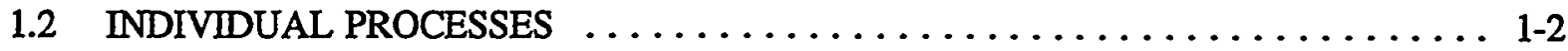

1.3 DESIGN AND PERFORMANCE ISSUES $\ldots \ldots \ldots \ldots \ldots \ldots \ldots \ldots \ldots \ldots \ldots \ldots$

1.4 OBJECTIVE AND SCOPE $\ldots \ldots \ldots \ldots \ldots \ldots \ldots \ldots \ldots \ldots \ldots \ldots \ldots \ldots \ldots \ldots$

1.5 SUMMARY OF LITERATURE SEARCH $\ldots \ldots \ldots \ldots \ldots \ldots \ldots \ldots \ldots \ldots \ldots$

1.6 STRUCTURE OF REPORT $\ldots \ldots \ldots \ldots \ldots \ldots \ldots \ldots \ldots \ldots \ldots \ldots \ldots \ldots$

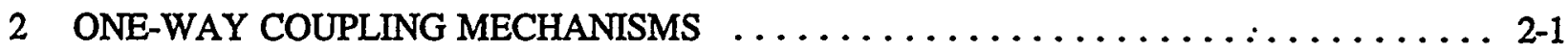

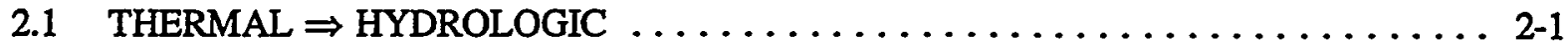

2.1.1 Vaporization and Condensation ...................... 2-2

2.1.2 Nonisothermal Enhancement of Vapor Diffusion $\ldots \ldots \ldots \ldots \ldots \ldots \ldots . . \ldots 2$

2.1.3 Dripping in Fractures . . . . . . . . . . . . . . . . . . . $2-6$

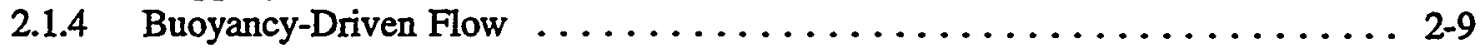

2.1.5 Computer Codes ........................... 2-14

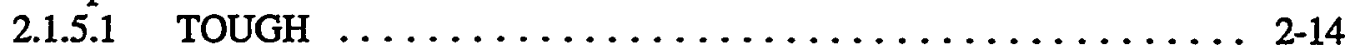

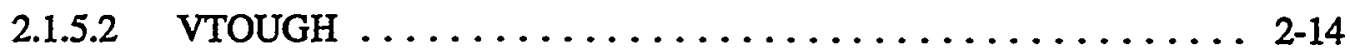

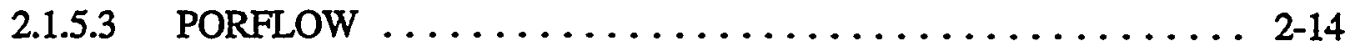

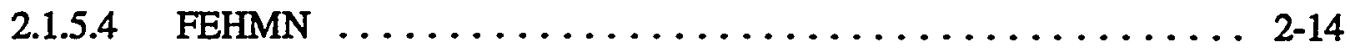

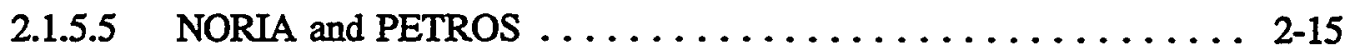

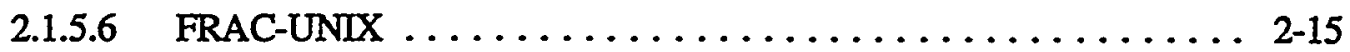

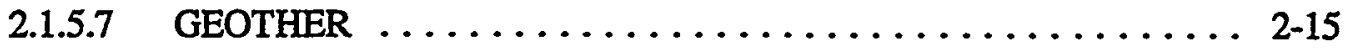

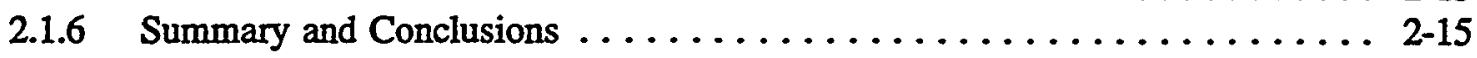

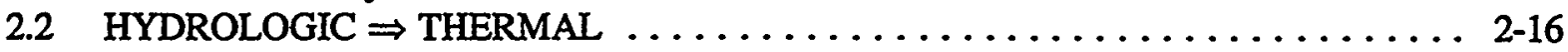

2.2.1 Heat Pipe ............................ $2-16$

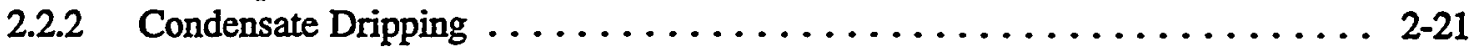

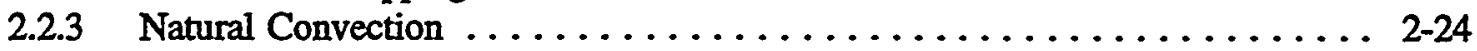

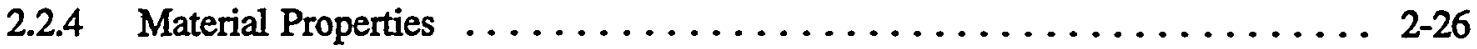

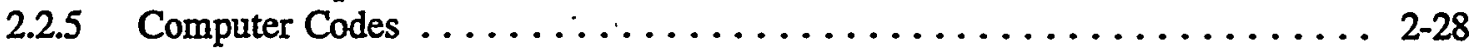

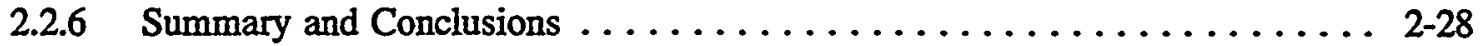

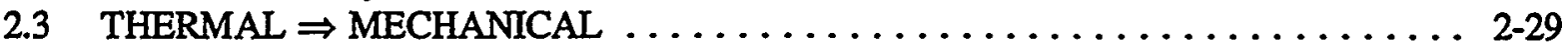

2.3.1 Mechanical Properties and Material Strength ................. 2-29

2.3.2 Thermally-Induced Stresses $\ldots \ldots \ldots \ldots \ldots \ldots \ldots \ldots \ldots \ldots \ldots \ldots \ldots$

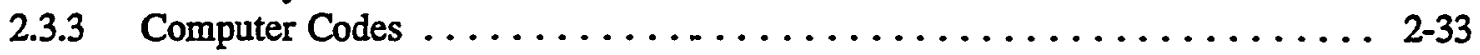

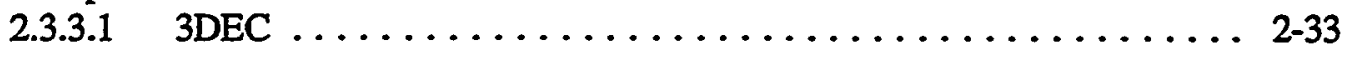

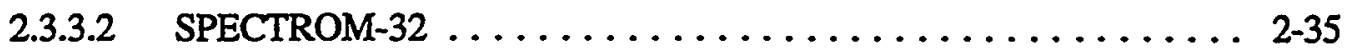

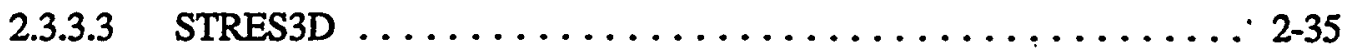




\section{CONTENTS (Cont'd)}

Section

Page

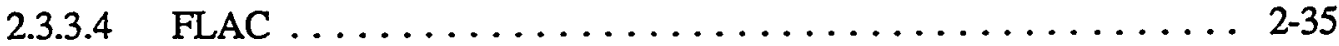

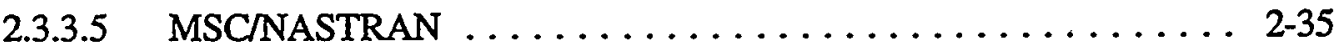

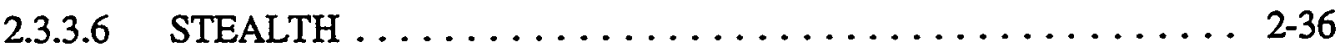

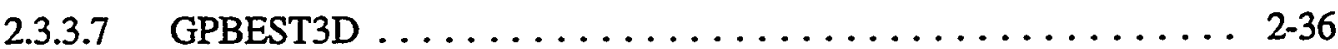

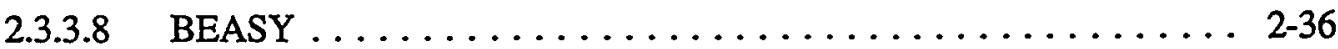

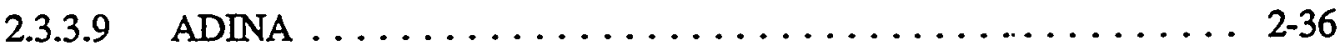

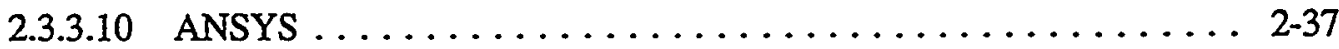

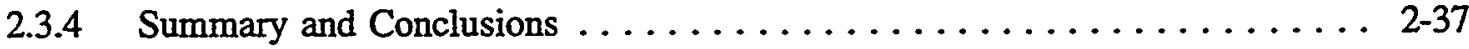

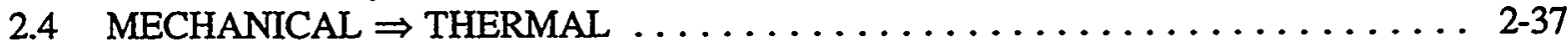

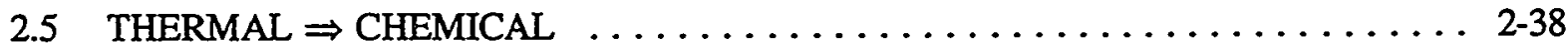

2.5.1 Thermal Effects on Equilibrium Chemistry $\ldots \ldots \ldots \ldots \ldots \ldots \ldots \ldots . \ldots \ldots$

2.5.1.1 Equilibrium Constants $(\log \mathrm{K}) \ldots \ldots \ldots \ldots \ldots \ldots . \ldots \ldots . \ldots \ldots$

2.5.1.2 Activity Coefficients ...................... 2-40

2.5.2 Temperature Effects on Nonequilibrium Chemistry . . . . . . . . . . 2-41

2.5 .3 Computer Codes .......................... 2-42

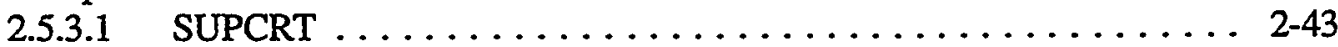

2.5.3.2 PHREEQE ......................... 2.43

2.5.3.3 WATEQ ........................ 2-44

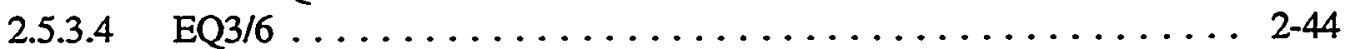

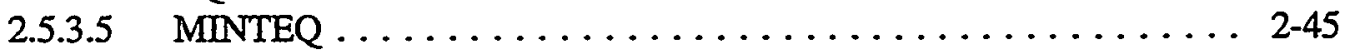

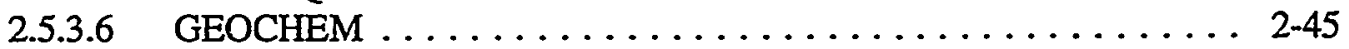

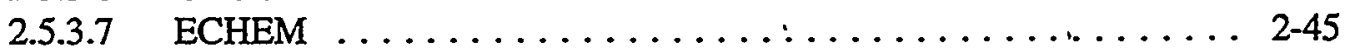

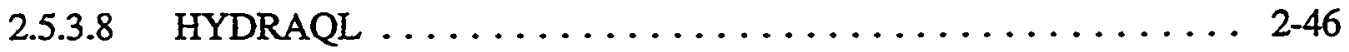

2.5.3.9 The Geochemist's Workbench . . . . . . . . . . . . 2-46

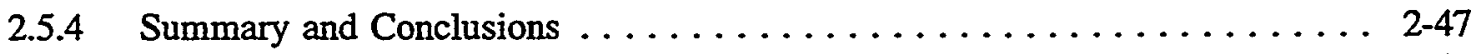

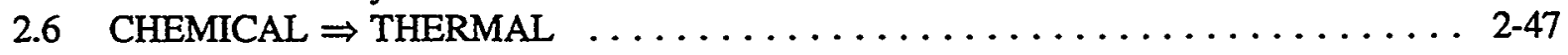

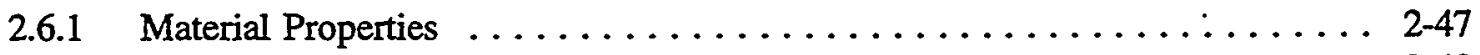

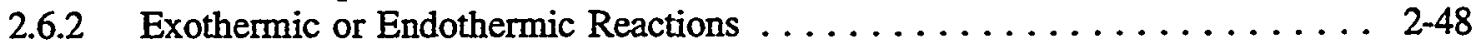

2.6 .3 Computer Codes ... . . . . . . . . . . . . . . . . . . 2-48

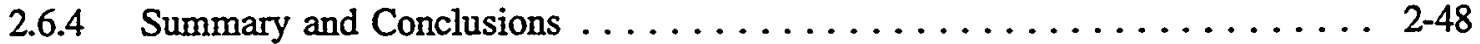

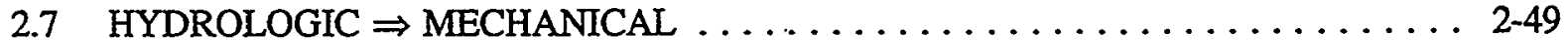

2.7.1 Mechanical Properties . . . . . . . . . . . . . . . . . . 2-49

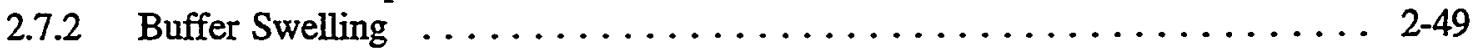

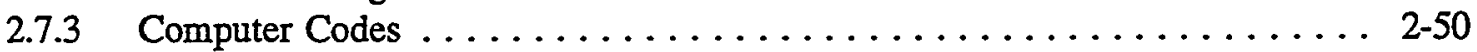

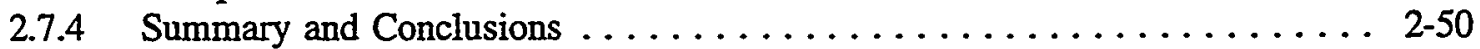

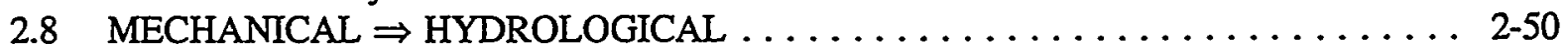

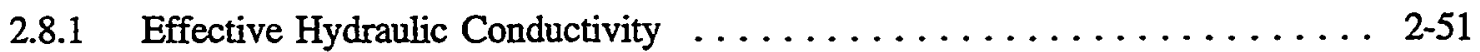

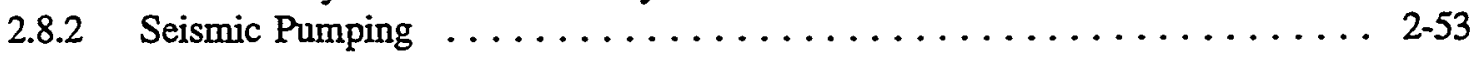

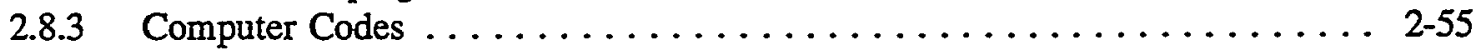

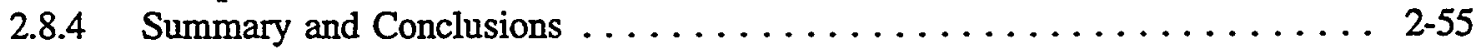

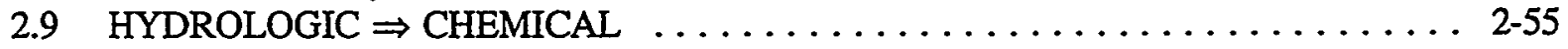

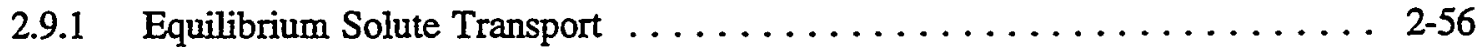

2.9 .2 Nonequilibrium Solute Transport $\ldots \ldots \ldots \ldots \ldots \ldots \ldots \ldots \ldots . \ldots \ldots$ 


\section{CONTENTS (Cont'd)}

Section

Page

2.9.2.1 Two-Region Transport .................. 2-60

2.9.2.2 Two-Site Transport ..................... 2-51

2.9.2.3 Stochastic Models ........................ 2-62

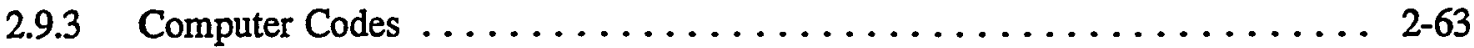

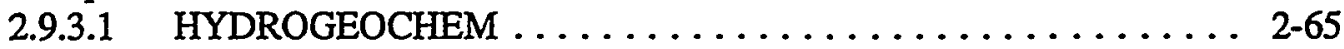

2.9.3.2 DYNAMIX ....................... 2-66

2.9.3.3 CHEQMATE ....................... 2-66

2.9.3.4 TRANQL ......................... 2-66

2.9.3.5 FASTCHEM ......................... 2-67

2.9.3.6 CTM ............................ 2-68

2.9.3.7 CHEMTRN ......................... 2-68

2.9.3.8 CHMTRNS $\ldots \ldots \ldots \ldots \ldots \ldots \ldots \ldots \ldots \ldots \ldots \ldots \ldots \ldots . \ldots \ldots$

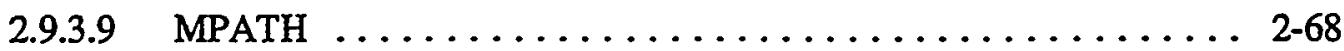

2.9.3.10 NEFTRAN, TRACR3D, and PORFLOW . . . . . . . . . . 2-69

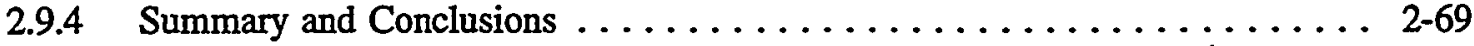

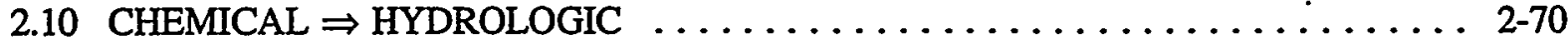

2.10.1 Mineral Deposition on Fracture Surfaces . . . . . . . . . . . . 2-70

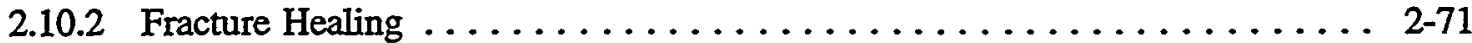

2.10.3 Chemical Osmosis ......................... 2-71

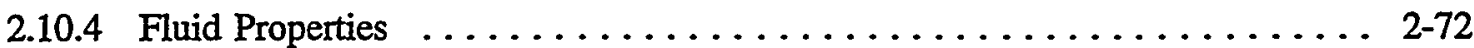

2.10 .5 Computer Codes ... . . . . . . . . . . . . . . . . . . . . . . 2-77

2.10 .6 Summary and Conclusions $\ldots \ldots \ldots \ldots \ldots \ldots \ldots \ldots \ldots \ldots \ldots \ldots \ldots \ldots$

2.11 MECHANICAL $\Rightarrow$ CHEMICAL $\ldots \ldots \ldots \ldots \ldots \ldots \ldots \ldots \ldots \ldots \ldots \ldots \ldots \ldots$

2.11.1 Pressure Effects on Chemical Equilibrium . . . . . . . . . . . . 2-79

2.11.2 Pressure Effects on Nonequilibrium Chemistry . . . . . . . . . . 2-82

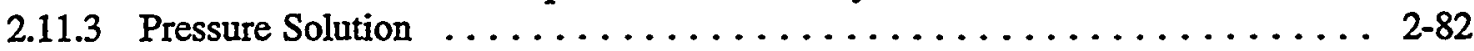

2.11 .4 Computer Codes . . . . . . . . . . . . . . . . . . . . 2-83

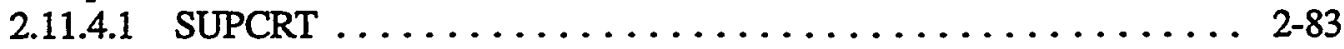

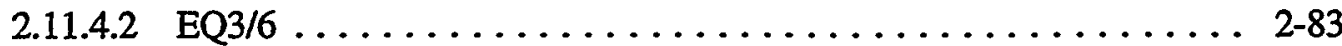

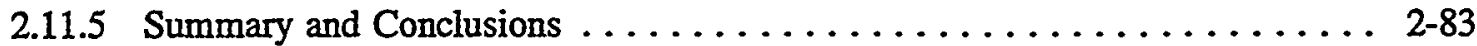

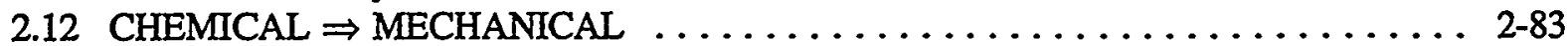

2.12.1 Chemical Effects on Rock Fracture Propagation . . . . . . . . . . . . . 2 2-84

2.12.2 Degradation of Mechanical Properties Due to Chemical Action . . . . . . . 2-85

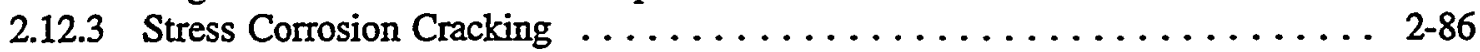

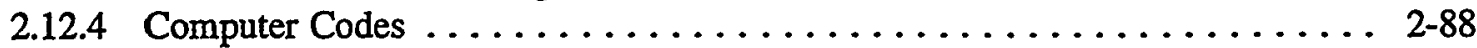

2.12.5 Summary and Conclusions $\ldots \ldots \ldots \ldots \ldots \ldots \ldots \ldots \ldots \ldots \ldots \ldots$

3 THREE-WAY COUPLED PROCESSES $\ldots \ldots \ldots \ldots \ldots \ldots \ldots \ldots \ldots \ldots \ldots \ldots$

3.1 THERMAL-HYDROLOGIC-MECHANICAL COUPLING $\ldots \ldots \ldots \ldots \ldots \ldots \ldots \ldots$. $\ldots \ldots$

3.1.1 DECOVALEX International Project $\ldots \ldots \ldots \ldots \ldots \ldots \ldots \ldots \ldots \ldots \ldots \ldots \ldots$

3.1.2 Computer Codes for THM Modeling $\ldots \ldots \ldots \ldots \ldots \ldots \ldots \ldots \ldots \ldots \ldots \ldots$

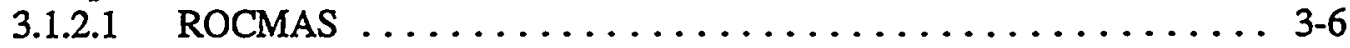

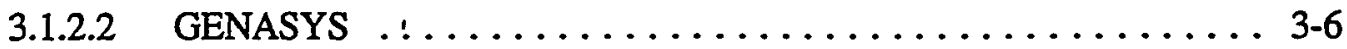




\section{CONTENTS (Cont'd)}

Section

Page

3.1.2.3 THAMES $\ldots \ldots \ldots \ldots \ldots \ldots \ldots \ldots \ldots \ldots \ldots \ldots . \ldots \ldots$

3.1.2.4 FEHMS .......................

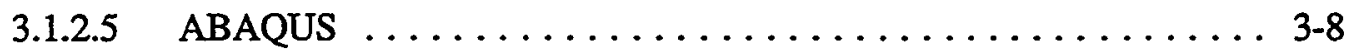

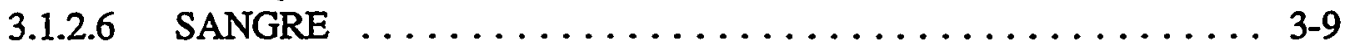

3.1.2.7 UDEC . . . . . . . . . . . . . . . . . . . 3-9

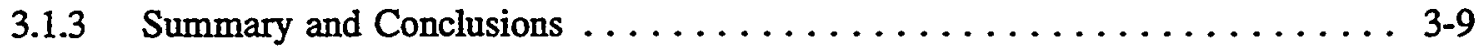

3.2 THERMAL-HYDROLOGIC-CHEMICAL COUPLING . . . . . . . . . . . . . 3-9

3.2.1 Effects of Coupling on Material Properties and Media Characteristics . . . . . 3-10

3.2.2 Constitutive Couplings . . . . . . . . . . . . . . . . . . $3-12$

3.2 .3 Computer Codes ........................... 3 .13

3.2.3.1 CHMTRNS $\ldots \ldots \ldots \ldots \ldots \ldots \ldots \ldots \ldots \ldots \ldots \ldots \ldots \ldots \ldots . \ldots \ldots$

3.2.3.2 THCC .......................... 3-14

3.2.3.3 Codell and Murphy $(1992) \ldots \ldots \ldots \ldots \ldots \ldots . \ldots \ldots$. $\ldots \ldots \ldots$

3.2.3.4 HYDROGEOCHEM . . . . . . . . . . . . . . . . . 3-14

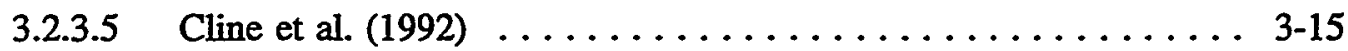

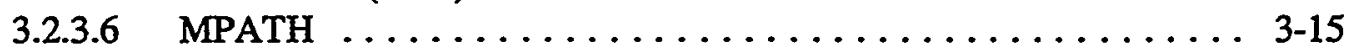

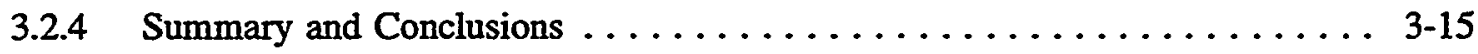

4 FOUR-WAY COUPLED PROCESSES $\ldots \ldots \ldots \ldots \ldots \ldots \ldots \ldots \ldots \ldots \ldots \ldots \ldots$

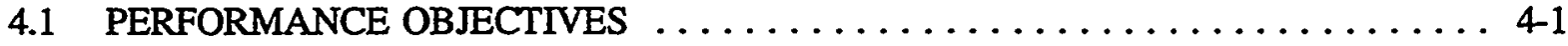

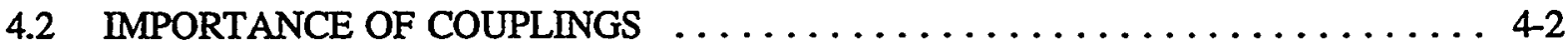

4.3 SUMMARY AND CONCLUSIONS $\ldots \ldots \ldots \ldots \ldots \ldots \ldots \ldots \ldots \ldots \ldots \ldots$

5 SUMMARY AND RECOMMENDATIONS $\ldots \ldots \ldots \ldots \ldots \ldots \ldots \ldots \ldots \ldots \ldots \ldots$

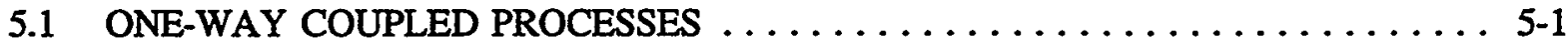

5.2 THREE-WAY COUPLED PROCESSES $\ldots \ldots \ldots \ldots \ldots \ldots \ldots \ldots \ldots \ldots \ldots \ldots$

5.3 FOUR-WAY COUPLED PROCESSES $\ldots \ldots \ldots \ldots \ldots \ldots \ldots \ldots \ldots \ldots \ldots$

6 REFERENCES $\ldots \ldots \ldots \ldots \ldots \ldots \ldots \ldots \ldots \ldots \ldots \ldots \ldots \ldots \ldots \ldots \ldots \ldots \ldots$

APPENDIX A. THERMAL PROCESSES $\ldots \ldots \ldots \ldots \ldots \ldots \ldots \ldots \ldots \ldots \ldots \ldots \ldots \ldots$

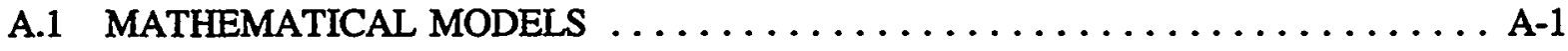

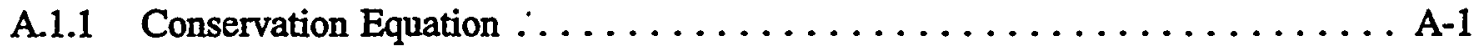

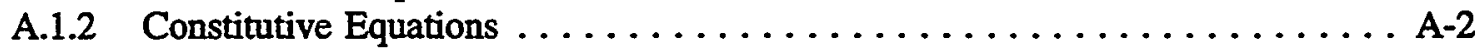

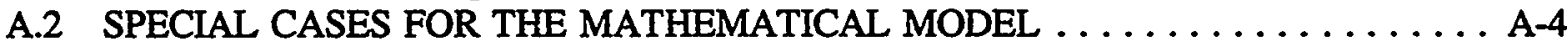

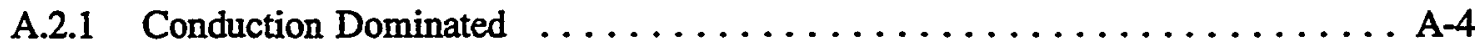

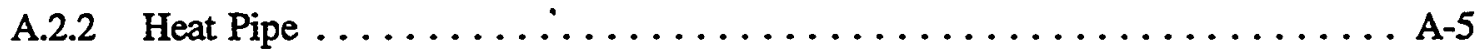

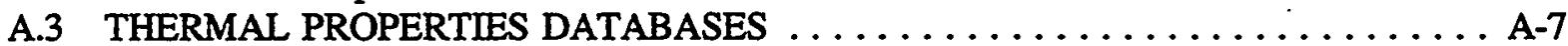

A.4 COUPLINGS WITH OTHER PROCESSES $\ldots \ldots \ldots \ldots \ldots \ldots \ldots \ldots \ldots \ldots \ldots \ldots$ 


\section{CONTENTS (Cont'd)}

Section

Page

APPENDIX B. HYDROLOGIC PROCESSES $\ldots \ldots \ldots \ldots \ldots \ldots \ldots \ldots \ldots \ldots \ldots \ldots$

B.1 A MATHEMATICAL MODEL $\ldots \ldots \ldots \ldots \ldots \ldots \ldots \ldots \ldots \ldots \ldots \ldots \ldots$. $\ldots \ldots \ldots$

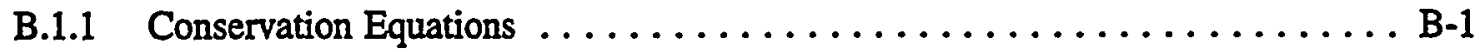

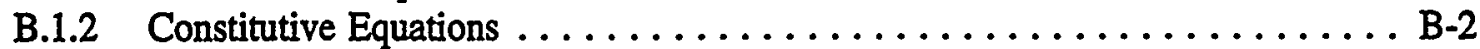

B.1.3 Material Properties . . . . . . . . . . . . . . . . . . . B-3

B.2 DATABASES FOR HYDROLOGIC PROPERTIES OF YUCCA MOUNTAIN . . . . . B-4

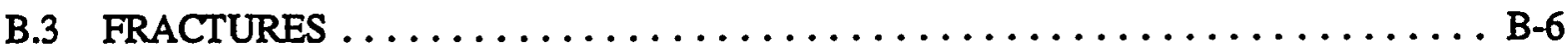

B.4 COUPLINGS WITH OTHER PROCESSES $\ldots \ldots \ldots \ldots \ldots \ldots \ldots \ldots \ldots \ldots \ldots \ldots$

APPENDIX C. MECHANICAL PROCESSES $\ldots \ldots \ldots \ldots \ldots \ldots \ldots \ldots \ldots \ldots \ldots \ldots$

C.1 CONTINUUM MATERIAL BEHAVIOR $\ldots \ldots \ldots \ldots \ldots \ldots \ldots \ldots \ldots \ldots \ldots$ C-1

C.2 ROCK JOINT BEHAVIOR . . . . . . . . . . . . . . . . . . C-3

C.3 MATHEMATICAL MODELING OF MECHANICAL PROCESSES . . . . . . . . C-5

C.3.1 Continuum Analysis $\ldots \ldots \ldots \ldots \ldots \ldots \ldots \ldots \ldots \ldots \ldots \ldots \ldots$

C.3.2 Discontinuum Analysis $\ldots \ldots \ldots \ldots \ldots \ldots \ldots \ldots \ldots \ldots \ldots \ldots \ldots$

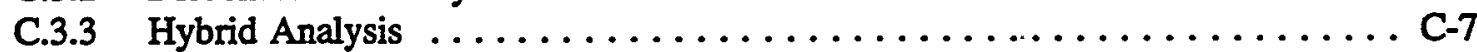

C.4 COUPLING WTTH OTHER PROCESSES $\ldots \ldots \ldots \ldots \ldots \ldots \ldots \ldots \ldots \ldots \ldots$

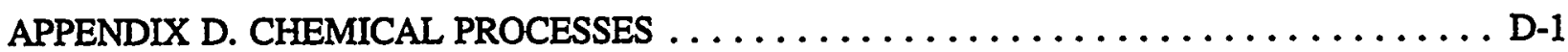

D.1 EQUILIBRIUM CHEMICAL PROCESSES $\ldots \ldots \ldots \ldots \ldots \ldots \ldots \ldots \ldots \ldots$ D-1

D.2 NONEQUILIBRIUM CHEMICAL PROCESSES $\ldots \ldots \ldots \ldots \ldots \ldots \ldots \ldots \ldots \ldots$ D 4

D.3 REACTION PROGRESS MODELS $\ldots \ldots \ldots \ldots \ldots \ldots \ldots \ldots \ldots \ldots \ldots \ldots \ldots \ldots \ldots$

D.4 THERMODYNAMIC DATABASES $\ldots \ldots \ldots \ldots \ldots \ldots \ldots \ldots \ldots \ldots \ldots \ldots \ldots$

D.5 COUPLING WITH OTHER PROCESSES $\ldots \ldots \ldots \ldots \ldots \ldots \ldots \ldots \ldots \ldots \ldots \ldots$ D $.9 \ldots$ 



\section{FIGURES}

Figure

Page

1-1 Diagrams of uncoupled and coupled processes (Tsang, 1987a, 1991) . . . . . . . 1-3

1-2 Types of coupled processes (Tsang, 1987a) $\ldots \ldots \ldots \ldots \ldots \ldots \ldots \ldots \ldots \ldots$

2-1 An axisymmetric model of a geologic repository (Verma and Pruess, 1988) . . . . . . 2-4

2-2 A 2D, cartesian model of a geologic repository (Pollock, 1986) . . . . . . . . . . . 2-4

2-3 Liquid saturation and temperature distributions through the center of a repository from ground surface $($ depth $=0 \mathrm{~m})$ to water table $($ depth $=500 \mathrm{~m})$ at time $=$ $(10,50,100,200,1000)$ years, see Figure $2-2$ (Pollock, 1986) . . . . . . . . . . 2-5

2.4 Illustration of a drift with vertical emplacement of a waste container in a fractured porous medium $\ldots \ldots \ldots \ldots \ldots \ldots \ldots \ldots \ldots \ldots \ldots \ldots \ldots$

2-5 Illustration of a dry zone and a condensation zone with condensate dripping in fractures for short times (above) and longer times (below) $\ldots \ldots \ldots \ldots \ldots \ldots \ldots . . \ldots$

2-6 Buoyancy driven convection patterns around a saturated repository (Wang and Tsang, 1980) .................................. 2-10

2-7 Illustration of convective circulation pattern for the gas phase (Pollock, 1986) . . . . . 2-10

2-8 Critical Rayleigh number (for the Benard problem) versus dimensionless permeability from Darcy's limit to the viscous fluid limit $\ldots \ldots \ldots \ldots \ldots \ldots \ldots \ldots$ 2-12

2-9 Gas flux at 100 yrs after waste emplacement showing flow away from repository primarily due to diffusion of water vapor (Tsang and Pruess, 1987) $\quad \ldots \ldots \ldots$ 2-13

2-10 Gas flux at 100 yrs after waste emplacement neglecting molecular diffusion of water vapor (Tsang and Pruess, 1987) . . . . . . . . . . . . . . . 2-13

2-11 Schematic of heat-transfer regimes in a plane perpendicular to the axis of the waste package (not to scale) (Pruess etial., 1990a) . . . . . . . . . . . . . . 2-17

2-12 Numerically-simulated liquid saturation $(S)$, pressure $(P)$, temperature $(T)$, and air-mole-fraction (Y) profiles at various times (Doughty and Pruess, 1988). A heat pipe is evident by a temperature "plateau." Reprinted from the International Journal of Heat and Mass Transfer, 31, C. Doughty and K. Pruess, A semianalytical solution for heat-pipe effects near high-level nuclear waste packages buried in partially saturated geological media, 79-90, copyright 1988, with permission from Pergamon Press Ltd., Headington Hill Hall, Oxford OX3 OBW, UK . . . . . . . . 2-19

2-13 Temperature profiles showing a lack of a plateau near $100^{\circ} \mathrm{C}$, hence the absence of a heat pipe. Calculations based on an explicit fracture model and an effective continuum model using hydrologic properties representative of Topopah Spring densely welded tuff (adapted from Pruess et al., 1990b) . . . . . . . . . . 2-20

2-14 Plan view (a) and cross-sectional view (b) along boreholes P2 and P3 of the LLNL' G-tunnel heater tests showing locations of thermocouples, fractures and the heater (adapted from Ramirez et al., 1991a, pg 11 and pg 90) . . . . . . . . . . 2-22

2-15 Temperature history of selected thermocouples above the heater (see Figure 2-14 for thermocouple locations) (Lin et al., 1991; also Ramirez et al., 1991a, where an apparent error in the $\mathrm{x}$-axis scaling has been corrected) $\ldots \ldots \ldots \ldots \ldots \ldots \ldots \ldots$

2-16 Temperature history of selected thermocouples below the heater (see Figure 2-14 for thermocouple locations) (Lin et al., 1991; also Ramirez et al., 1991a) 


\section{FIGURES (Cont'd)}

Figure

Page

2-17 2D model of the overlying medium at a geologic repository (Kulacki and Keyhani, 1987)

(Note: The geometric dimensions $d$ and $D$ are defined here and used in Figure 2-18) . . 2-25

2-18 Experimental results which reflect repository conditions for a saturated, homogeneous, isotropic medium (see Figure 2-17) (Rajen and Kulacki, 1987) . . . . . 2-25

2-19 Experimental apparatus used to measure the thermal conductivity of Apache Leap tuff as a function of water content (Rasmussen et al., 1990) . . . . . . . . . . . . 2-27

2-20 Thermally-induced normal fracture displacement (a) for horizontal fracture zone just beneath the repository and (b) of vertical fracture zone intersecting repository. A positive sign indicates opening displacement (Shen and Stephansson, 1990a) . . . . . 2-32

2-21 Thermal-mechanical environment in the vicinity of (a) waste emplacement drift and (b) tuff main access drift at the proposed repository beneath Yucca Mountain (Hardy and Bauer, 1992). Reprinted from: W.R. Wawersik (ed.), 33rd US Rock Mechanics Symposium-Proceedings, Santa Fe, New Mexico, 8-10 June 1992. 1992. 1157 pp. Hfl.150/US \$85.00. Please order from: „A.A. Balkema, Old Post Road, Brookfield, Vermont 05036 (telephone: 802-276-3162; telefax: 802-276-3837) . . . . . . . . . 2-34

2-22 Illustration of axial, radial, and circumferential fractures around a circular

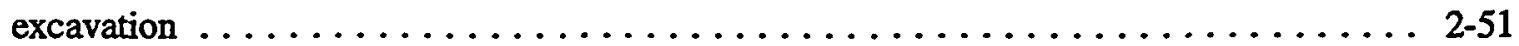

2-23 Illustration of normal stresses in the circumferential $\left(\sigma_{\theta \theta}\right)$ and radial $\left(\sigma_{\pi}\right)$ orientation around a circular excavation in a linear, isotropic, homogeneous rock mass medium in a hydrostatic stress field $\ldots \ldots \ldots \ldots \ldots \ldots \ldots \ldots$

2-24 Water table response for isotropic permeability and for local vertical permeability enhancement (10 to $10^{3}$ ) within a 100-m-wide anisotropic region centered on a normal fault simulating a zone of vertical fracturing. Results are based on a $1-\mathrm{m}$ slip allowed on a fault located $250 \mathrm{~m}$ below the water table (Carrigan et al., 1991) . . . . 2-54

2-25 Water density as a function of temperature for different salinities $(m=$ moles $\mathrm{NaCl} / \mathrm{kg} \mathrm{H} \mathrm{H}_{2} \mathrm{O}$ ) at a constant pressure of $10 \mathrm{MPa} \approx 100 \mathrm{~atm}$ (adapted from Forster and

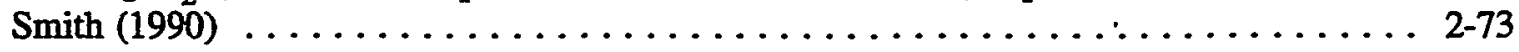

2-26 Dynamic viscosity of water as a function of salinity for constant $P=10 \mathrm{MPa} \approx 100 \mathrm{~atm}$ (adapted from Forster and Smith, 1990)

2-27 Shifting of the vapor-liquid line for pure water (solid line) with the addition of $10 \mathrm{wt} \% \mathrm{NaCl}$ (dotted line) and $25 \mathrm{wt} \% \mathrm{NaCl}$ (dashed line). The critical points are also shown. The straight lines are isochores representing constant density fluids with P-T. The numbers represent the density in $\mathrm{g} / \mathrm{cm}^{3}$ (adapted from Roedder, 1984) . . 2-75

2-28 P-T plot of isocompositional curves for the gas phase in equilibrium with the liquid phase for the system $\mathrm{H}_{2} \mathrm{O}-\mathrm{CO}_{2}$. The critical curve for the system extends from the critical point for water $\left(374{ }^{\circ} \mathrm{C}\right.$ and 220 bars) to lower temperatures and higher pressures. The minimum critical temperature occurs at approximately $266^{\circ} \mathrm{C}$ and about 2150 bars (adapted from Takenouchi and Kennedy, 1964) . . . . . . . . . 2-76

2-29 Water diffusivity of $\mathrm{K}^{+}$and $\mathrm{Cl}^{-}$as a function of moisture content for a silty clay loam (adapted from Laryea et al., 1982). . . . . . . . . . . . . . . . . . . 2-78

2-30 Crack propagation stress of synthetic and natural quartz samples plotted versus chemical environment (Dunning et al., 1984) . . . . . . . . . . . . . . . 


\section{FIGURES (Cont'd)}

Figure

Page

2-31 Resistance to SCC as a function of applied stress. Metals are commercial stainless

steel in boiling $42 \% \mathrm{MgCl}_{2}$ at $154{ }^{\circ} \mathrm{C}$ (adapted from Fontana and Greene, 1978) . . . 2-88

2-32 Crack growth rate $(\mathrm{m} / \mathrm{s})$ as a function of stress intensity $\left(\mathrm{MPa} \mathrm{m}^{1 / 2}\right)$ for Type 304 stainless steel (adapted from Chung et al., 1985) . . . . . . . . . . . . . 2-89

2-33 Susceptibility of Fe-18Cr-xNi alloys to SCC in boiling $42 \% \mathrm{MgCl}_{2}$ as a function of nickel content (adapted from Copson, 1959) . . . . . . . . . . . . . . 2-90

3-1 Coupled THM analyses using ROCMAS showing (a) finite element model of the source environment and (b) variation of fluid inflow to the heater borehole as a function of time after heater is turned on (Noorishad et al., 1984) . . . . . . . . . . . . . 3-3

3-2 Pressure and aperture profiles in the fracture generated by ROCMAS for various durations, and temperature profiles along heater midplane (Noorishad et al., 1984) . . . 3-4

3-3 Coupled thermo-hydrologic-mechanical (THM) benchmark problem for DECOVALEX. (a) Block geometry showing locations of monitoring points and applied heat flux. (b) Zone discretization for discrete element code UDEC . . . . . . . 3-5

3-4 Coupled THM benchmark problem for DECOVALEX. (a) Temperature contours assuming only conduction heat transfer through the rock medium. (b) Temperature contours taking into account conduction, as well as the effect of forced convection

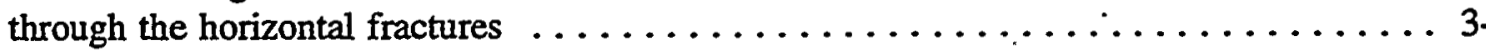

4-1 Illustration of the important processes and couplings during the operation, containment, and isolation periods for anticipated processes and events at the proposed repository. The right column is a subset of the left where only primary couplings which feed into the processes of primary regulatory interest are shown $\ldots \ldots 47$

4-2 Schematic diagram of major factors affecting radionuclide transport from geologic repositories (Wang et al., 1983) $\ldots \ldots \ldots \ldots \ldots \ldots \ldots \ldots \ldots \ldots \ldots \ldots \ldots \ldots$

A-1 Relative thermal power of $\mathrm{HLW}$ showing the importance of fission products at short times and actinides at long times (Verma and Pruess, 1988; among others) . . . . A A-4

A-2 Typical temperature histories of waste package components and host rock near emplaced spent fuel canister (O'Neal et al., 1984) $\ldots \ldots \ldots \ldots \ldots \ldots \ldots \ldots \ldots \ldots$

A-3 Comparison of experimental measurements of the emissivity of oxidized zircaloy (spent fuel rods) (Hohorst et al., 1990) $\ldots \ldots \ldots \ldots \ldots \ldots \ldots \ldots \ldots \ldots \ldots \ldots \ldots$

B-1 East-west cross-sectional illustration highlighting the different hydrogeologic units in the unsaturated zone at Yucca Mountain (adapted from DOE, 1988)

B-2 Effective continuum permeability as a function of saturation showing influences of matrix and fracture flow (Montazer and Wilson, 1984; Domenico and Schwarz, 1990; among others) $\ldots \ldots \ldots \ldots \ldots \ldots \ldots \ldots \ldots \ldots \ldots \ldots \ldots \ldots \ldots \ldots \ldots$

B-3 Comparison of calculated and measured liquid saturation profiles for various steady-state, 1D recharge fluxes (Buscheck \& Nitao, 1992). Calculated values based on an effective continuum model and measured values are from the Reference Information Base (RIB) (DOE, 1990)

D-1 Distinctions between classes of chemical reactions (adapted from Rubin, 1983) . . . . D-2 



\section{TABLES}

Table

Page

2-1 Rock mass thermal conductivity of selected units at Yucca Mountain (DOE, 1990)

3-1 Summary of driving potentials, fluid pressure, temperature, and species concentration, and how they are expected to generate a flux of fluid, heat or species

4-1 Assessment of the importance of coupled THMC processes for anticipated processes and events, for performance objectives associated with the operations time period of the proposed HLW repository (10 CFR 60.111) . . . . . . 42

4-2 Assessment of the importance of coupled THMC processes for anticipated processes and events, for performance objectives associated with the containment time period at the proposed HLW repository (10 CFR 60.113) $\ldots \ldots \ldots 42$

4-3 Assessment of the importance of coupled THMC processes for anticipated processes and events, for performance objectives associated with the isolation time period at the proposed HLW repository (10 CFR 60.112 and 40 CFR Part 191) ... 4-2

B-1 Summary of hydrogeologic properties relevant to Yucca Mountain (adapted from DOE, 1988) $\ldots \ldots \ldots \ldots \ldots \ldots \ldots \ldots \ldots \ldots \ldots \ldots \ldots$

D-1 Examples of tabulations of thermodynamic data (modified from

Serne et al., 1990) 
- 


\section{NOMENCLATURE}

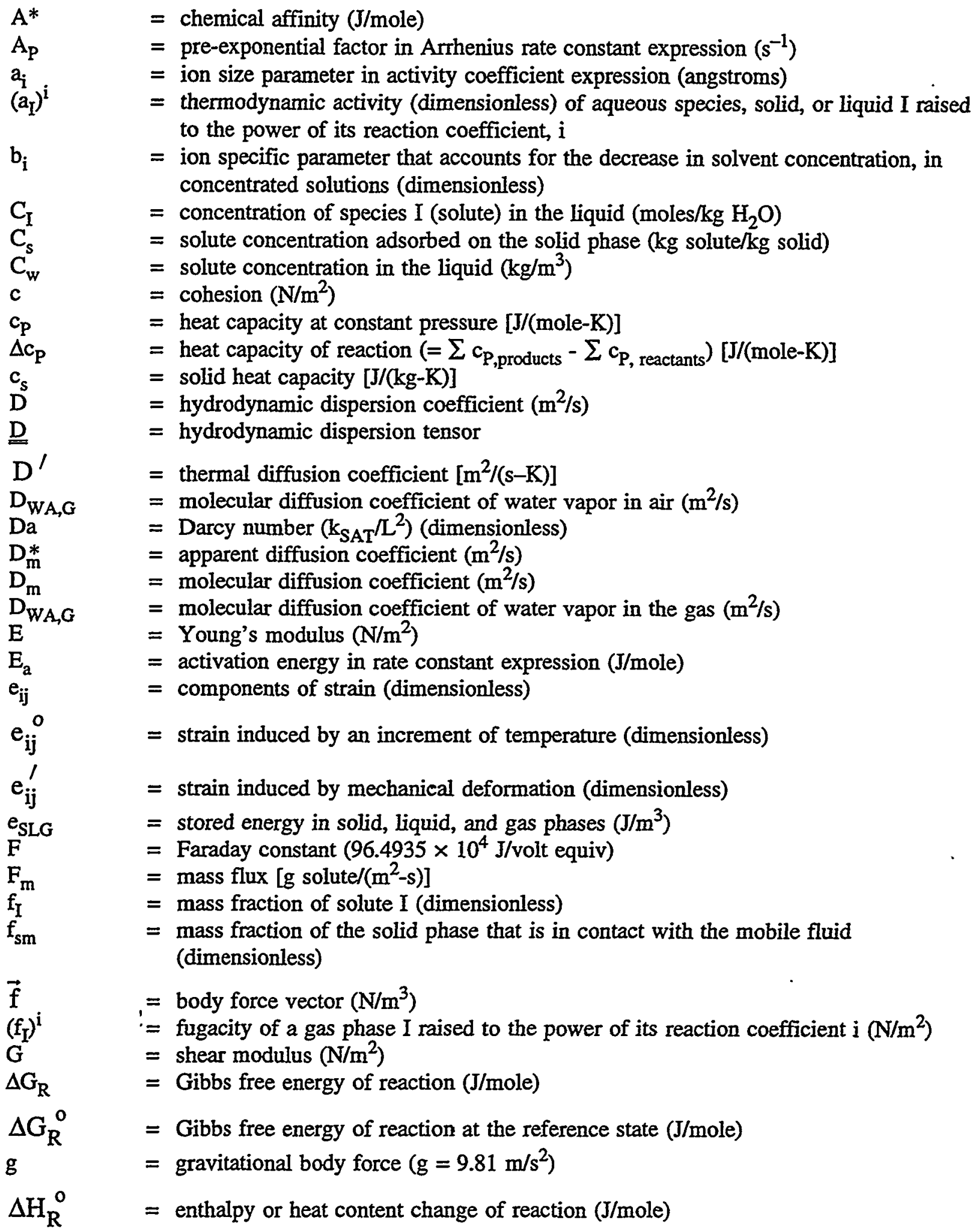




\section{NOMENCLATURE (Cont'd)}

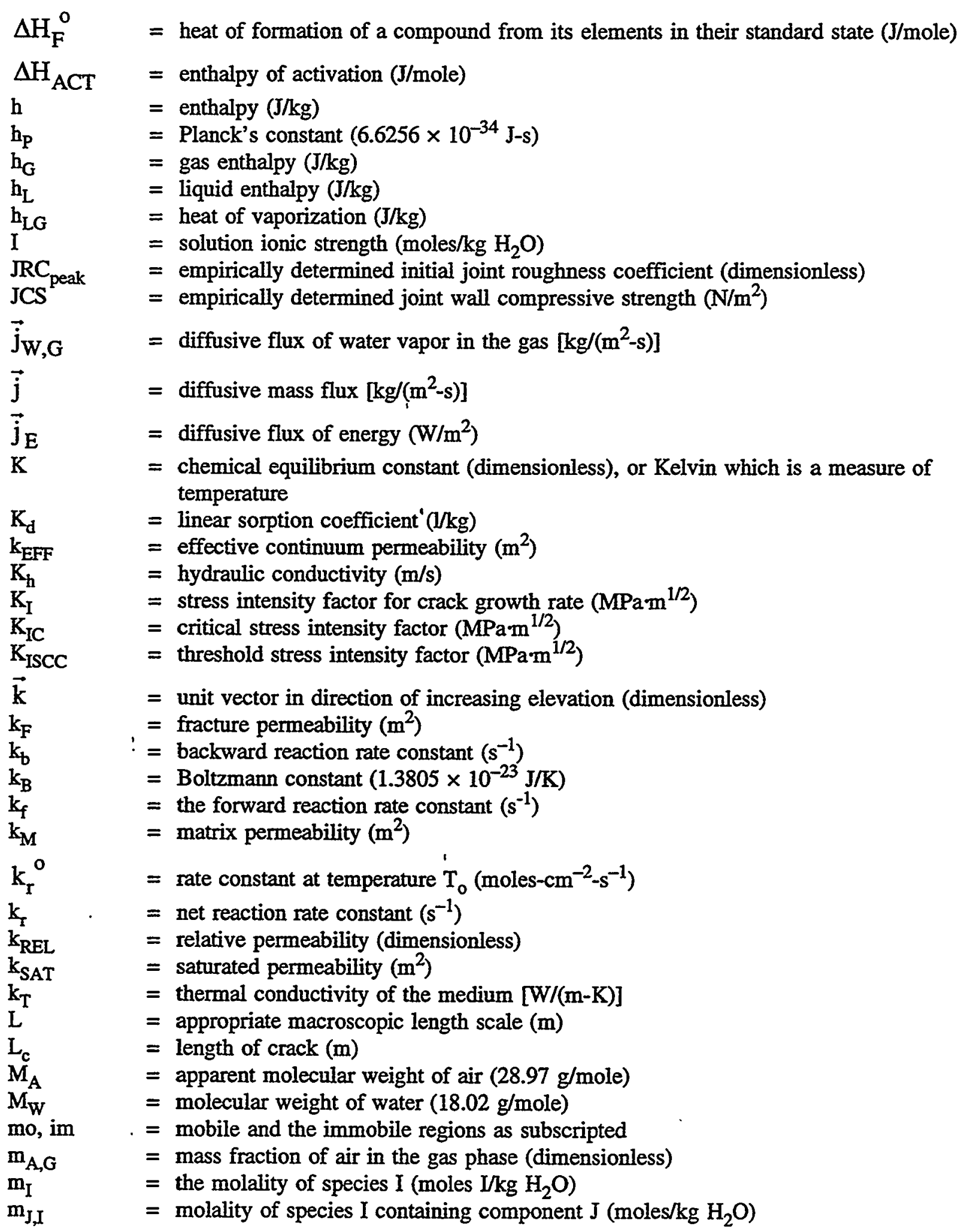




\section{NOMENCLATURE (Cont'd)}

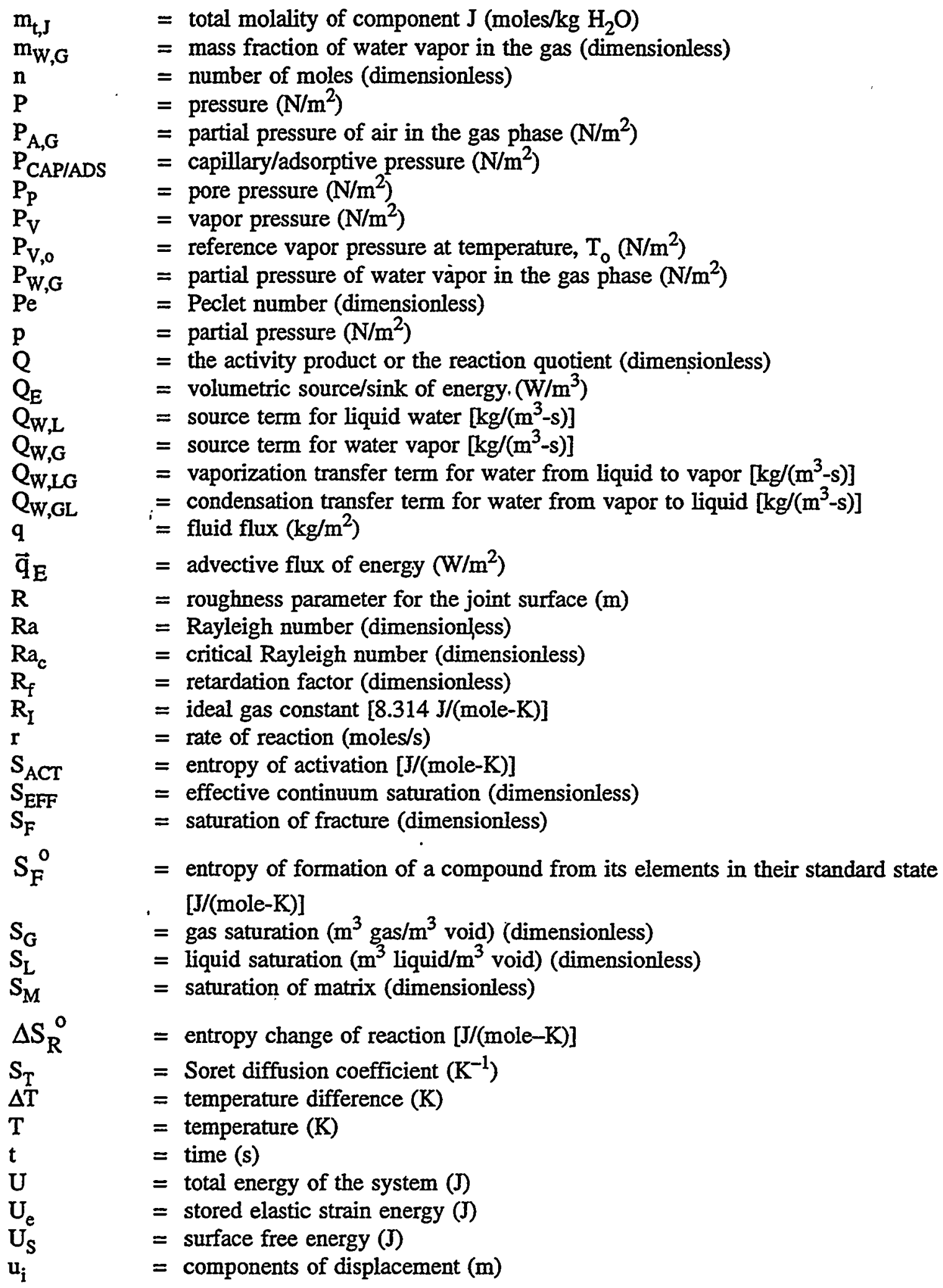




\section{NOMENCLATURE (Cont'd)}

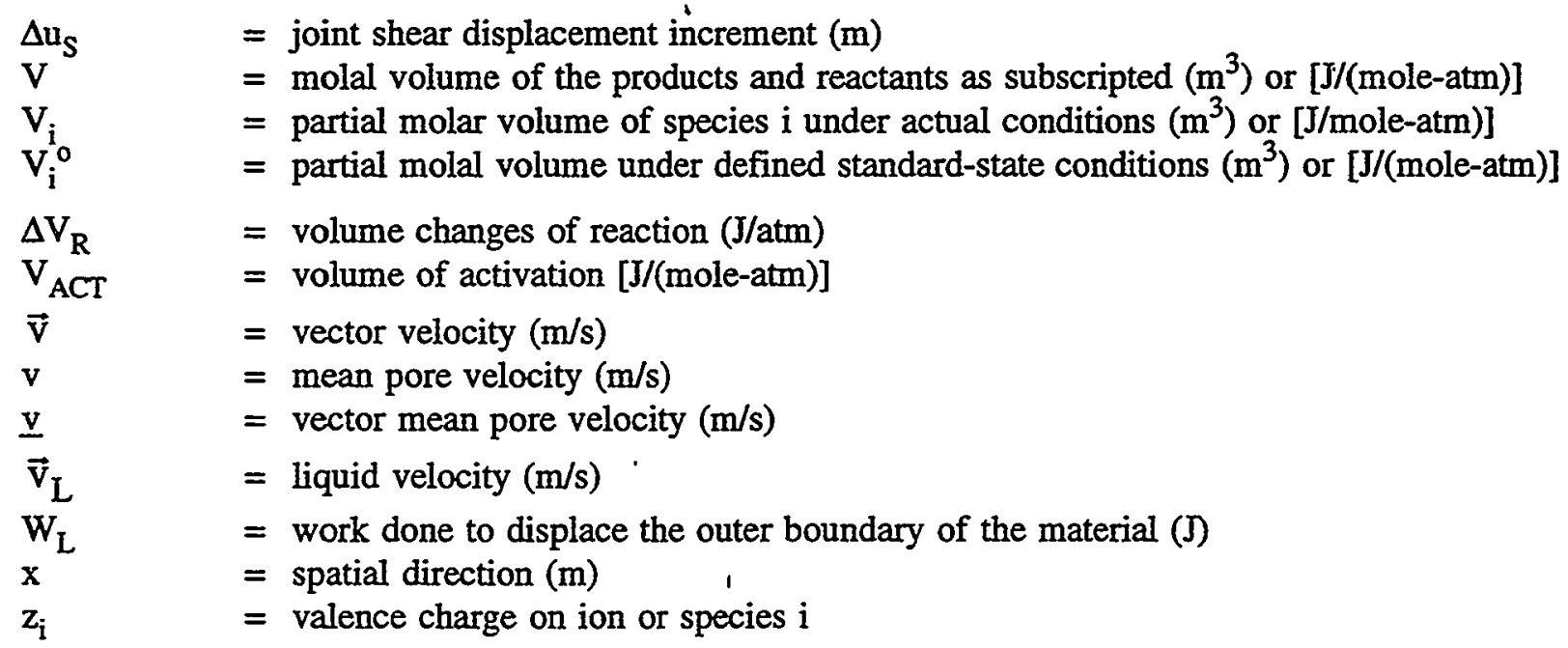

\section{Greek:}

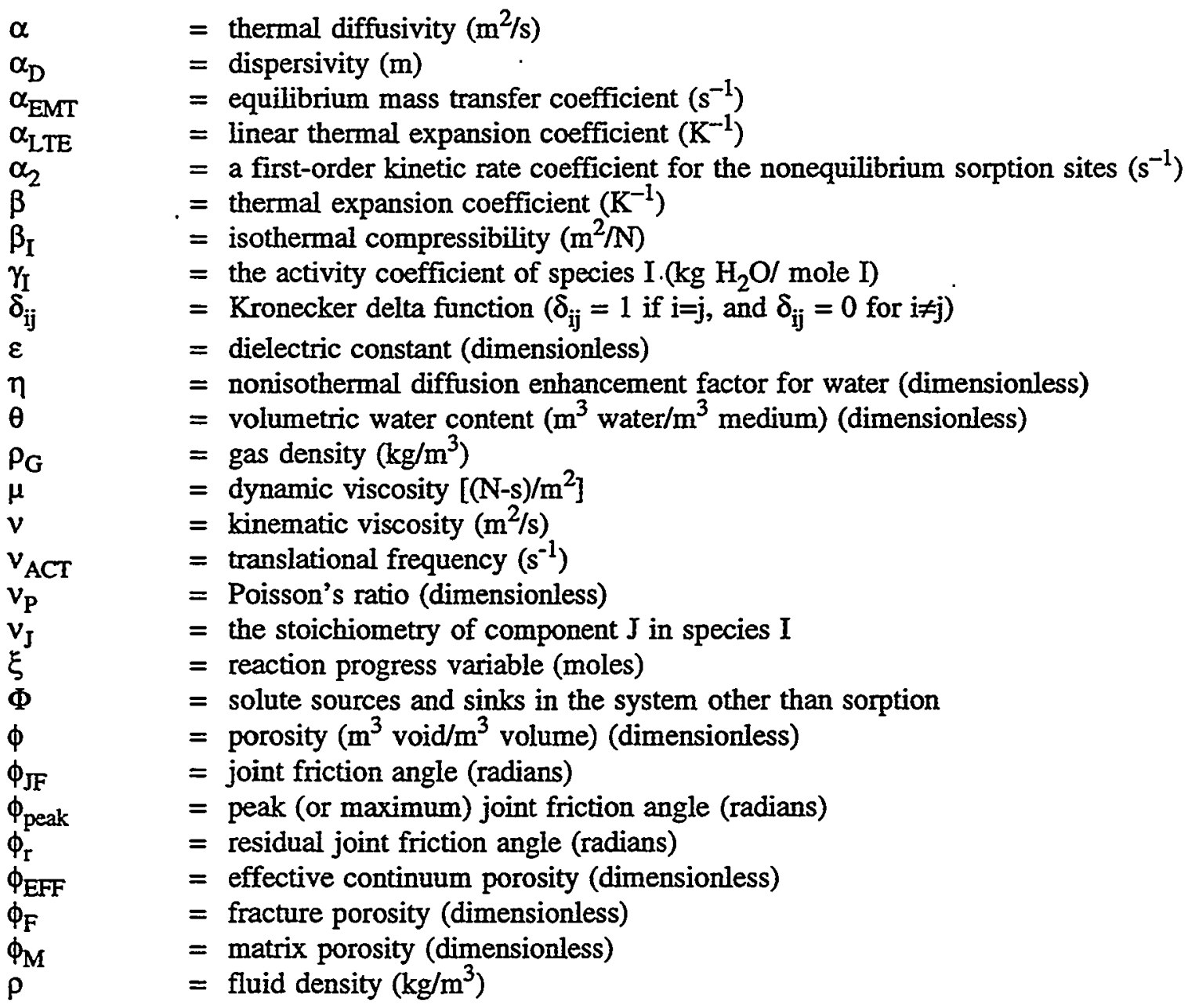




\section{NOMENCLATURE (Cont'd)}

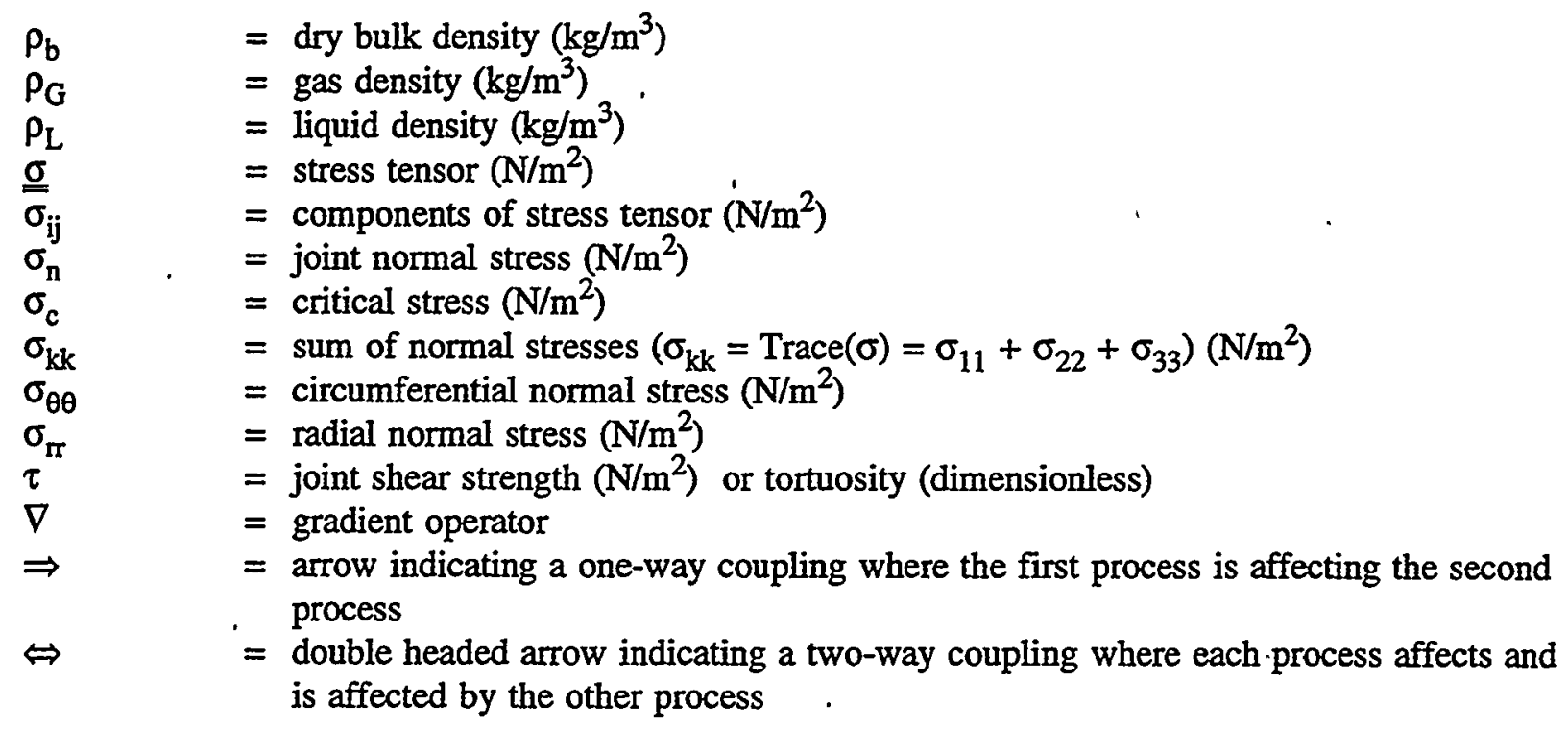

\section{Acronyms}

\begin{tabular}{|c|c|}
\hline APD & - areal power density \\
\hline ASME & - American Society of Mechanical Engineers \\
\hline CHEQMATE & - CHemical EQuilibrium with MigrAtion and Transport Equations \\
\hline CNWRA & - Center for Nuclear Waste Regulatory Analyses \\
\hline CTM & - Chemical Transport Model \\
\hline DECOVALEX & - DEvelopment of COupled models and their VALidation against EXperiments \\
\hline DHLWM & - Division of High-Level Waste Management \\
\hline DOE & - Department of Energy \\
\hline $\mathrm{ECM}$ & - equivalent continuum model \\
\hline EDL & - electrical double layer \\
\hline EPA & - Environmental Protection Agency \\
\hline EPRI & - Electric Power Research Institute \\
\hline FEHMS & - Finite Element Heat Mass Stress \\
\hline FFPS & - Free-face pressure solution \\
\hline FLAC & - Fast Lagrangian Analysis of Continua \\
\hline GENASYS & - Geotechnical ENgineering Analysis SYStem \\
\hline GROA & - geologic repository operations area \\
\hline HLW & - High-Level Waste \\
\hline IPS & - intergranular pressure solution \\
\hline LANL & - Los Alamos National Laboratory \\
\hline LBL & - Lawrence Berkeley Laboratory \\
\hline LLNL & - Lawrence Livermore National Laboratory \\
\hline MPATH & - Multiple Reaction Path \\
\hline NEA & - Nuclear Energy Agency \\
\hline NMSS & - Nuclear Material Safety and Safeguards \\
\hline NNWSI & - Nevada Nuclear Waste Storage Investigations \\
\hline
\end{tabular}




\section{NOMENCLATURE (Cont'd)}

$\begin{array}{ll}\text { NRC } & \text { - Nuclear Regulatory Commission } \\ \text { NTIS } & \text { - National Technical Information Service } \\ \text { ORNL } & \text { - Oak Ridge National Laboratory } \\ \text { PHREEQE } & \text { - pH-REdox-EQuilibrium-Equations } \\ \text { PNL } & \text { - Pacific Northwest Laboratory } \\ \text { REV } & \text { - representative elemental volume } \\ \text { ROCMAS } & \text { - ROC Mass Analysis Scheme } \\ \text { SCC } & - \text { Stress corrosion cracking } \\ \text { SNL } & - \text { Sandia National Laboratories } \\ \text { TGE } & - \text { thermogravitational effect } \\ \text { THAMES } & - \text { Thermal, Hydraulic And MEchanical System } \\ \text { THCC } & - \text { Thermo-Hydro-Chemical Coupling } \\ \text { THM } & - \text { thermal, hydrological, and mechanical } \\ \text { THMC } & - \text { thermal, hydrologic, mechanical, and chemical } \\ \text { USGS } & - \text { U.S. Geological Survey }\end{array}$




\section{ACKNOWLEDGEMENTS}

The authors would like to thank S.M. Hsiung, B. Sagar, A.C. Bagtzoglou, B.W. Leslie, G.A. Cragnolino, P.C. Lichtner, and W.C. Patrick for their technical reviews of this document. We also thank M.S. Nataraja, R.B. Codell, R.G. Wescott, B.N. Jagannath, W.J. Boyle, S.J. Chern and other reviewers at the U.S. Nuclear Regulatory Commission (NRC) for their helpful comments. The authors are also thankful to Cathy Cudd for skillful typing and formatting, Pedro (Pete) Garza for preparing the figures, and Curtis (Corky) Gray and Don Moore for editing this report.

This report was prepared to document work performed by the Center for Nuclear Waste Regulatory Analyses (CNWRA) for the NRC under Contract NRC-02-88-005. The activities reported here were performed on behalf of the NRC Office of Nuclear Material Safety and Safeguards (NMSS), Division of High-Level Waste Management (DHLWM). The report is an independent product of the CNWRA and does not necessarily reflect the views or regulatory position of the NRC. 


\section{EXECUTIVE SUMMARY}

In the design and performance assessment of the proposed high-level nuclear waste (HLW) repository at Yucca Mountain, it is expected that numerous computational simulations will be performed considering thermal, hydrologic, mechanical, and chemical (THMC) processes over a variety of spatial scales (microns to kilometers) and temporal scales (0 to $10,000 \mathrm{yrs}$ ). There appears to be a general consensus in the literature concerning the importance of considering coupled THMC processes [e.g., Wang et al., 1983; Tsang, 1987a, 1991; 10 CFR Part 60 (Code of Federal Regulations), 1992]. For example, the emplacement of heat-generating radioactive waste is expected to influence the performance of the repository, and thermal loads are specifically referenced in the U.S. Nuclear Regulatory Commission (NRC) regulations [10 CFR 60.133(i)] as needing to be considered for mechanical processes $(T \Rightarrow M)$ and groundwater flow $(\mathrm{T} \Rightarrow \mathrm{H})$. [The arrow symbol $(\Rightarrow)$ is used to indicate a coupling between two processes, where the first process is the affecting process, and the second process is the affected process.]

A recent NRC staff technical position outlines an acceptable methodology for systematically considering thermal loads and thermally-induced mechanical, hydrologic, and chemical processes (Nataraja and Brandshaug, 1992). The primary purpose of the NRC technical position is to outline an acceptable method to comprehensively, systematically, and logically understand and evaluate THMC responses for the design and performance assessment of a geologic repository. This report provides subjective assessments and evaluations of the current understanding, state of modeling (especially as implemented in computer codes), and anticipated importance of THMC processes. It is important to note that these assessments and evaluations are based on the current understanding of the processes and data uncertainties, and may have to be modified as new data and information emerge.

In Section 1, the concept of coupled processes is reviewed, especially the work of Tsang (1987a). The terminology of one-way, two-way, three-way, and four-way coupled processes is introduced. This report is structured so that attention is focused on the coupling mechanisms between two processes, where one process is the affecting process, and the second is the affected process (i.e., one-way coupling).

The four individual processes ( $T, H, M$, and $C$ ) are discussed in the appendices (A, B, C and D, respectively) because this information is considered background material, whereas the coupled effects are the primary focus of this report. As background, each process can be distinguished by a set of state variables (which are not necessarily independent). For example, thermal processes are described by the temperature and heat-flux fields. Hydrologic processes are described by the distribution of fluids (especially liquid water, water vapor, and air) and fluid fluxes. Mechanical processes are described by the stress, strain, and displacement fields. Chemical processes are described by the distributions, fluxes, and interactions of species. A coupling between processes is described by cause-and-effect relationships between the ptocesses. For example, a $\mathrm{T} \Rightarrow \mathrm{H}$ coupling indicates that the temperature field influences the flow of fluids (e.g., the redistribution of in situ groundwater). Similarly, $H \Rightarrow M$ indicates that the distribution and flow of fluids affect the state of stress, strain, and/or displacements.

In Section 2, each of the 12 unique one-way couplings $(T \Rightarrow H, T \Rightarrow M, T \Rightarrow C, H \Rightarrow T, H \Rightarrow M$, $H \Rightarrow C, M \Rightarrow T, M \Rightarrow H, M \Rightarrow C, C \Rightarrow T, C \Rightarrow H, C \Rightarrow M)$ are discussed. The discussion emphasizes the coupling mechanisms between processes. For example, the mechanisms of vaporization, molecular diffusion, condensation, condensate dripping in fractures, and thermally-driven buoyant flows are discussed for the $\mathrm{T} \Rightarrow \mathrm{H}$ one-way coupling. Another example is that the $\mathrm{C} \Rightarrow \mathrm{H}$ coupling may be due to changes in fracture surface mineralogy, fracture healing/plugging, fluid flow due to chemical osmosis driving 
forces, and chemically-induced changes in hydrologic properties (e.g., viscosity, vapor pressure). Wherever possible, conceptual and mathematical models are presented to describe the coupling mechanisms. Because the mathematical models which describe coupled processes are frequently complex, a review of computer codes is summarized for each of the one-way couplings. The review of computer codes summarizes the capabilities and modeling approaches, primarily as reported in the literature. It is estimated that computer codes will be used extensively to perform coupled analyses for design and performance assessment of the geologic repository. As such, Section 2 summarizes conceptual models, mathematical models, and computer codes for each of the one-way couplings, and in doing so it constitutes the "backbone" of the report.

In Section 3, three-way coupled processes are discussed. Specifically, THM and THC processes are discussed, because they are considered potentially important to the proposed repository. For THM coupled processes, current benchmark and validation efforts within the international DECOVALEX (DEvelopment of COupled models and their VALidation against EXperiments) project are highlighted. For THC coupled processes, constitutive couplings are also discussed where gradients in driving potentials result in fluxes of either heat, fluid, and/or species.

In Section 4, four-way coupled processes are discussed, and a subjective assessment is presented of the importance of each of the one-way couplings. It is emphasized that the couplings are considered important if, and only if, they affect the design and/or performance of the repository. The performance objectives are stated in 10 CFR Part 60, especially Sections 60.111, 60.112, and 60.113 and the specific references to the "generally applicable environmental standards for radioactivity as may have been established by the Environmental Protection Agency (EPA)" [(60.111(a) and 60.112]. The EPA standards stem from 40 CFR Part 191 (Code of Federal Regulations) (1989), which places limits on the cumulative release of radionuclides, and sets limits on the annual dose to the public.

For discussion purposes, the performance objectives of the repository are considered in three time periods: (i) Operation (until closure), (ii) Containment (closure to 300-1,000 yrs), and (iii) Isolation (containment to $10,000 \mathrm{yrs}$ ). In addition, the assessment is restricted to anticipated processes and events (10 CFR 60.2). Each one-way coupling is subjectively assessed within this scope:

- anticipated processes and events;

- impacting performance objectives;

- at the proposed repository; and

- during either the operation, containment, or isolation time period.

The importance is subjectively assessed as being either primary, secondary, or tertiary. Each rating is defined as:

- primary = confidently expected to be important;

- secondary = potentially important; however, the available evidence suggests that the coupling may be neglected without significant errors;

- tertiary = confidently expected to be negligible. 
The criteria used in this assessment are subjective. The development of definitive objective criteria from 10 CFR Part 60 and 40 CFR Part 191 is considered worthwhile, but it is beyond the scope of this literature review.

The assessment is based on the currently available information and is expected to be updated as the knowledge of the processes and couplings improves through ongoing investigation and site characterization. Hence, this assessment is çonsidered as one step in an iterative process that can help to identify and prioritize design and site characterization activities. A literature review such as this is considered beneficial in that it represents a systematic approach to evaluating coupled processes. By being systematic, it reduces the potential of overlooking important couplings.

In Section 5, the findings of the literature review are summarized, and recommendations for future investigations are presented. Recommendations for future work are summarized in (i) the continuing development of conceptual and mathematical models, (ii) the performance of well-documented coupledeffects experiments, and (iii) the validation of models through comparison of model predictions and experimental observation. It is recommended that property measurement experiments be conducted for key thermal, hydrologic, mechanical, and chemical properties (such as being planned and performed in the site characterization activities). 'In addition, it is recommended that coupled-effects experiments be designed and conducted to assess the importance (or lack thereof) for coupled processes. A number of coupled processes are widely recognized as being important, and considerable resources are being devoted to improving the level of understanding of these processes. Other coupled processes, however, warrant further investigation, especially synergistic effects involving chemical reaction and mass transport under thermal loadings (THC) and mechanical stability of underground excavations under varying thermal and hydrologic conditions (THM).

A review, such as reported here, is useful in identifying which coupled effects will be important, hence which coupled effects will need to be investigated by the NRC in order to assess the assumptions, data, analyses, and conclusions in the design and performance assessment of a geologic repository. Although this work stems from regulatory interest in the design of the geologic repository, it should be emphasized that the repository design implicitly considers all of the repository performance objectives, including those associated with the time after permanent closure. The scope of this review is considered beyond previous assessments in that it attempts (with the current state-of-knowledge) to determine which couplings are important, and identify which computer codes are currently available to model coupled processes. 


\section{INTRODUCTION}

\subsection{STATE OF COUPLING}

Despite the similarity between an underground mine and a mined geologic repository system, substantial differences exist in the analysis and design practice of underground openings of these two types of facilities. The considerations for repository design are different from the underground mine design considerations, primarily due to factors such as the thermal load of heat-generating waste, the time and space scales required for effective waste isolation, and the legal and regulatory environment in which waste isolation is to be engineered and managed.

Yucca Mountain, in southern Nevada, has been designated by the United States Congress for characterization as a potential repository site for nuclear waste disposal. The geomechanical conditions at the site are characterized by a highly-fractured rock material with prominent vertical and sub-vertical jointing, and faults which transgress the site environs. One feature of the proposed site is that the host rock is partially saturated and is several hundred meters above the groundwater table. This feature is substantially different from proposed high-level waste (HLW) repositories in other countries, all of which are located in saturated environments (i.e., below the water table). Inevitably, in the partially-saturated condition, gas and/or vapor phases will be introduced into the system, consequently making the already complicated system even more complicated.

The emplacement of radioactive waste in a partially-saturated geologic repository will cause a major perturbation of the system involving thermal $(T)$, hydrologic $(H)$, mechanical $(M)$, and chemical $(C)$ processes. The fractured rock mass will be perturbed in two ways (Tsang, 1987a). First, the construction of the repository results in large cavities in the fractured rock mass, which changes the original stress distribution. This stress redistribution causes mechanical deformation of the rock, causing the closing or opening of existing fractures. Fracture opening or closing affects fluid flow and solute transport in the rock mass, which are particularly important to the performance of a geologic nuclear waste repository and its environment. Second, the nuclear waste represents a heat source that is active over an extended period of time. This thermal input induces buoyant fluid flow (convection) and rock expansion, which directly depend not only on the magnitude of temperature rise but also on the integrated heat input into the system. The rock expansion will cause the dilation, closure, shear, and propagation of fractures. The hydraulic conductivity of both rock matrix and rock fracture may also change. The thermal load may also cause the degradation of the mechanical properties of rock, the healing of rock fractures, and changes of the chemical sorption and retardation capabilities.

There appears to be a general consensus in the literature concerning the importance of considering coupled thermal, hydrologic, mechanical, and chemical (THMC) processes [e.g., Wang et al., 1983; Tsang, 1987a,b, 1991; 10 CFR Part 60 (Code of Federal Regulations), 1992]. For example, the emplacement of heat-generating radioactive waste is expected to influence the performance of the repository, and thermal loads are specifically referenced in the U.S. Nuclear Regulatory Commission (NRC) regulations [10 CFR 60.133(i)] as needing to be considered for mechanical processes $(T \Rightarrow M)$ and groundwater flow $(T \Rightarrow H)$ [The arrow symbol $(\Rightarrow)$ is used to indicate a coupling between two processes, where the first process is the affecting process, and the second process is the affected process]. A recent NRC staff technical position outlines an acceptable methodology for systematically considering thermal loads and thermally induced mechanical, hydrologic, and chemical processes (Nataraja and Brandshaug, 1992). The primary purpose of the NRC technical position is to outline an acceptable method 
to comprehensively, systematically, and logically understand and evaluate THMC responses for the design and performance assessment of a geologic repository.

The presence of heat in a partially-saturated geologic repository induces coupled THMC processes that can be complex. Coupling of the THMC processes implies that one process affects the initiation and progress of another. Therefore, under the coupled condition, the behavior of the repository cannot be predicted by considering each process independently.

Tsang (1987a, 1991) discusses the distinction between different degiees of coupling in order to clarify what is meant by coupled processes. Figure 1-1 schematically displays several possible interactions between processes. The fully uncoupled processes have negligible influence on one another and, therefore, can be evaluated independently. The sequential case implies that one process depends on the final state of another, so that the order in which they are evaluated becomes important. The one-way coupled processes reflect a continuing effect of one or more processes on the others, so that their mutual influences change over time. The two-way coupling (or feedback coupling) reveals a continuing reciprocal interaction between two different processes. Any combination of one-way and two-way couplings between three or more processes is called three-way or more couplings, respectively. In this report, the term "coupled phenomenon" refers to one-way or more couplings among the processes.

Among the four processes, there can be only twelve one-way couplings, six two-way couplings, four three-way couplings, and one four-way coupling. Tsang (1987a) has considered two-way, three-way, and four-way couplings, and provided examples of various coupling mechanisms, as listed in Figure 1-2. Besides displaying the various types of possible coupling among the four processes, Figure 1-2 also indicates the strength of coupling (stronger couplings shown as double lines, and weaker couplings shown as single lines). The results of Figure 1-2 are based on knowledge available in 1987, primarily on saturated systems, and may have to be modified as new data and information emerge.

In the nuclear waste geologic repository there are several time and geometric scales. The major time scales are preclosure construction and retrievability period, postclosure containment period, and postclosure isolation period as discussed in 10 CFR 60.102(d) and (e). The major geometric scales include waste package, drift, repository, and distance to accessible environment boundary scales. The importance of various couplings will depend upon the design of the engineered barriers, properties of the geologic medium, and the scale (both temporal and spatial) at which these phenomena are of interest.

\subsection{INDIVIDUAL PROCESSES}

In order to discuss coupled processes, the individual processes must be defined. For example, thermal processes are related to the temperature and heat flux fields; hydrologic processes are related to the distribution and fluxes of fluids (especially liquid water, water vapor, and air); mechanical processes are related to the stress, strain, and displacement fields; and chemical processes are related to the distributions and fluxes of chemical components and species. Wang et al. (1983) considered the transport of radionuclides separately from chemical processes; however, in this work, it is considered an aspect of chemical processes, where a radionuclide is a particular species.

The mathematical formulations of individual THMC processes are summarized respectively, in Appendices A, B, C, and D and are briefly discussed here. In the appendices, it is noted that the processes are well understood for some conditions but not for many pertinent conditions. For example, the 
Uncoupled Processes

Fully Uncoupled

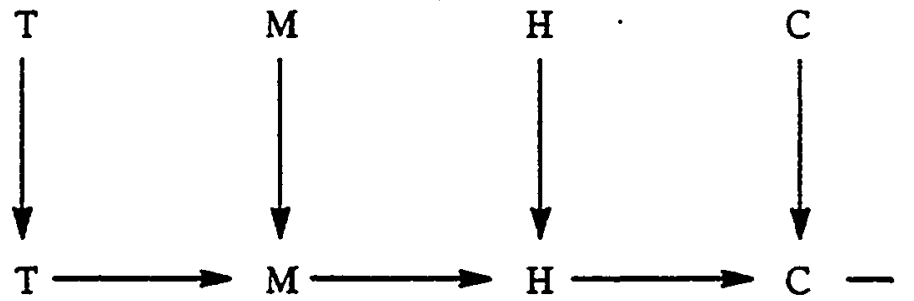

Coupled Processes

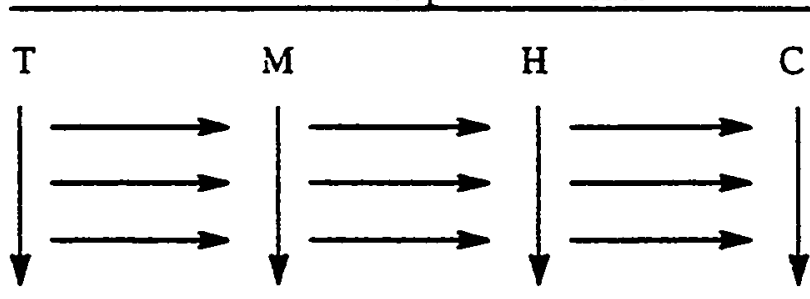

Two-way Coupling

(Feedback Coupling)

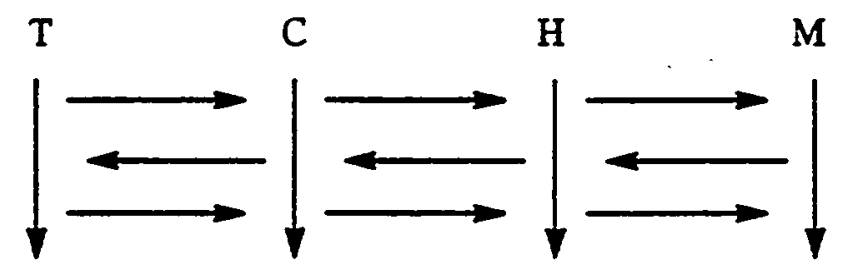

Note: $\mathrm{T}=$ Thermal, $\mathrm{M}=$ Mechanical, $\mathrm{H}=$ Hydrologic, $\mathrm{C}=$ Chemical.

Figure 1-1. Diagrams of uncoupled and coupled processes (Tsang, 1987a, 1991). 


\begin{tabular}{lll}
\hline No. & Type & \multicolumn{1}{c}{ Example } \\
\hline 1. & $\mathrm{~T}=\mathrm{C}$ & phase changes \\
2. & $\mathrm{T}=\mathrm{H}$ & buoyancy flow \\
3. & $\mathrm{T}=\mathrm{M}$ & thermally induced fractures \\
4. & $\mathrm{H}=\mathrm{C}$ & solution and precipitation \\
5. & $\mathrm{H}=\mathrm{M}$ & hydraulic fracturing \\
6. & $\mathrm{C}-\mathrm{M}$ & stress corrosion \\
& $\mathrm{T}$ & chemical reactions and transport \\
7. & $\bigwedge_{\mathrm{H}}$ & in hydrothermal systems \\
& $\mathrm{C}$
\end{tabular}

8.

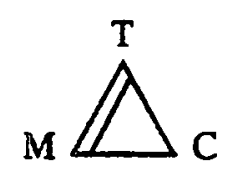

9.

10.

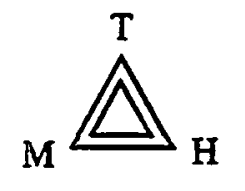

thermomechanical effects with change of mechanical strengths due to thermochemical transformation

thermally induced hydromechanical behavior of fractured rocks

11. hydromechanical effects (in fractures) that may influence chemical transport

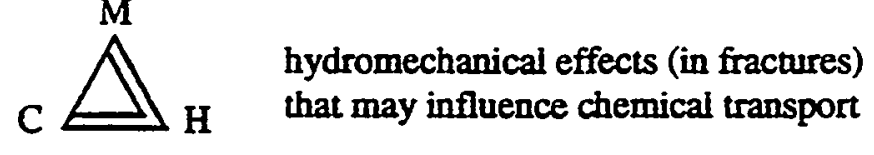 chemical reactions and transport in fractures under thermal and hydraulic loading

Note: $\mathrm{T}=$ Thermal, $\mathrm{M}=$ Mechanical, $\mathrm{H}=$ Hydrologic, $\mathrm{C}=$ Chemical. A single line indicates weak coupling; a double line indicates strong coupling.

Figure 1-2. Types of coupled processes (Tsang, 1987a). 
mathematical models for hydrologic processes in a homogeneous and isotropic saturated porous medium are better understood than those in a fractured, partially-saturated medium, which is the expected condition at the proposed repository. Characterization of fractures and fracture networks provides one major difficulty in predicting hydrologic processes. Although effective porous medium models for fractured media seem to be promising, this approach is accurate only as long as the hydrologic processes are sufficiently slow so that the fracture and matrix are in hydrologic equilibrium. Effective continuum models may not be appropriate in low-permeability units where episodic, fracture-dominated flow may dominate the hydrology.

Heat conduction is expected to be the dominant heat-transfer mechanism in the geologic medium at the proposed repository site and can be predicted through well-established mathematical models. However, the effective thermal conductivity can be influenced by rock-joint separation (mechanical processes) in addition to being influenced by the degree of saturation of the medium (hydrologic processes). In the waste package, the heat transfer may be due to a combination of conduction, natural convection, and thermal radiation. Natural convection and thermal radiation can be influenced by the geometry and thermal boundary conditions, which can be complex in the waste package.

The behavior of a continuous medium under mechanical processes has been covered in the literature. However, the mechanical modeling technique for a discontinuous medium (such as a fractured rock mass) is at an early stage of development. This may be further complicated by thermal stresses (thermal processes) and changes in joint properties due to the degree of saturation (hydrologic processes) and chemical alterations (chemical processes).

The chemical processes pertinent to the proposed HLW repository at Yucca Mountain may be described by either equilibrium or kinetic chemical models, depending on the reactions and the time scale of interest. The modeling of local equilibrium chemistry of aqueous systems in a continuous medium at ambient temperatures (i.e., $20^{\circ} \mathrm{C}$ ) has been well developed. However, the presence of heat sources and discontinuities in the geologic medium provide complexities that are not well understood for the modeling of equilibrium chemical processes. The kinetic chemical processes are less understood at this time, and accurate modeling techniques are needed.

\subsection{DESIGN AND PERFORMANCE ISSUES}

The waste isolation concept for the repository consists of both engineered and natural barriers that act to safely isolate the waste. The engineered barrier system (EBS) consists of the waste package and the underground structure, and is designed to contain the waste for a period of 300 to 1,000 yrs following permanent closure. During the containment time period, the radiation and temperatures are high, and the waste packages within the EBS should provide substantially complete containment of the waste [10 CFR 60.113(a)(1)(i)(A)] and gradual release of radionuclides [10 CFR 60.133(a)(1)(i)(B)] after the containment period. The geologic setting of the site should also isolate the waste from the accessible environment. An important feature of the geologic setting is that it may experience changes due to seismicity, volcanism, and thermal loads during the long period of waste isolation. Furthermore, the geologic repository operations area (GROA), consisting of both surface and subsurface areas, will be designed for loads due to in situ stresses, natural phenomena, and thermally-induced phenomena (Nataraja and Brandshaug, 1992). The GROA design should preserve the option of waste retrieval during the retrievability period, and limit the radiation exposures and releases of radioactive materials to unrestricted areas until permanent closure has been completed. 


\subsection{OBJECTIVE AND SCOPE}

The objective of this literature review is to determine the state of the art in modeling of coupled THMC processes relevant to the proposed repository at Yucca Mountain, Nevada. Literature reviewed in this document have been selected primarily to enable the Center for Nuclear Waste Regulatory Analyses (CNWRA), and therefore the NRC, to develop the technical capability to review and/or independently verify the U.S. Department of Energy (DOE) submittals to the NRC for thermal effects on repository design and preclosure and postclosure performance.

Only a selected number of coupled analysis computer codes are described herein. In selecting these computer codes, the results of recent literature reviews (Kana et al., 1991; Tumer, 1991a) conducted at the CNWRA for the Seismic Rock Mechanics, and the Performance Assessment research projects have been utilized. No attempt has been made to provide an exhaustive list of coupled analysis computer codes. In addition, only a few of the codes have been used by the authors, and the summary of a code is based largely on the reported capabilities.

A review, such as reported here, is useful in identifying which coupled effects will be important, hence which coupled effects will need to be investigated by the NRC in order to assess the assumptions, data, analyses, and conclusions in the design and performance assessment of a geologic repository. Although this work stems from regulatory interest in the design of the geologic repository, it should be emphasized that the repository design implicitly considers all of the repository performance objectives, including those associated with the time after permanent closure. The scope of this review is considered beyond previous assessments in that it attempts (with the current state-of-knowledge) to determine which couplings are important, and identify which computer codes are currently available to model coupled processes.

\subsection{SUMMARY OF LITERATURE SEARCH}

A literature search was performed to determine the state of the art in the area of modeling coupled THMC processes in porous, fractured rock as it is pertinent to the proposed HLW repository. The electronic databases used in the search are listed here:

- National Technical Information Service (NTIS) (64-91/9112B1)

- COMPENDEX PLUS (1970-1991/Nov)

- GEOREF (1785-1991/Aug)

- GEOARCHIVE (74-91/Sept)

- FLUIDEX (73 - 90/Dec)

- ISMEC: MECHANICAL ENGINEERING (1973-91/Oct)

- CONFERENCE PAPERS INDEX (73-91/Nov)

- ENERGY SCIENCE \& TECHNOLOGY (74-91/Oct)

- ENERGYLINE (70-91/Oct)

- GEOBASE (1980-91/Aug)

- CHEMABSTRACTS (1967-1991) 
Four partially independent searches were conducted to cover each of the four areas with coupling to the remaining three areas. For example, a search was conducted with thermal as the primary topic, and another search used chemical as the primary topic. Because the number of citations was extremely large for certain cuiegories, the scope for each topic was adaptively adjusted (two searches are reported for both hydrologic and mechanical processes). The key words used are as follows.

For thermal:

(thermal or heat or geothermal) and (multiphase or multiOphase or 20phase or two(phase) and (fluid or fluids or hydraulic or hydrology?) and (porous or fract? or rock)

For hydrologic (the first time):

(multiphase or multiOphase or 20phase or two0phase) and (fluid or fluids or hydraulic or hydrology?) and (porous or fract? or rock) and (mechanical or elastic? or deformation or matrix or stress? or displacement or joint?)

For hydrologic (the second time):

((fluid or fluids) or hydraulic()conductivity or hydrology or hydrologic?) and (porous or fract? or joint?) and rock and (deform? or hydromechanical or hydro(mechanical or elastic?)

For mechanical (the first time):

(mechanical or elastic? or deformation or matrix or stress? or displacement or joint?) and (thermal or heat or geothermal) and (multiphase or multiOphase or 20phase or two(phase) and (fluid or fluids or hydraulic or hydrology?)

For mechanical (the second time): thermal()mechanical and (thermal()conductivity or model?) or (thermomechanical or thermo()mechanical) and model? or (pressure or stress) and (thermalOconductivity or heat capacity) and (rock? or stone or granite or limestone or shale or sandstone)

For chemical:

chemi? and geochemi? and thermochemi?

and (thermal or heat or geothermal or thermo?)

and (hydrology? or hydraulic or hydrogeo? or geohydro? or fluid)

and (porous or fract? or rock)

and (mechanical or elastic? or deformation or matrix or stress or displacement or joint)

Over 1,000 abstracts were requested and reviewed, with over 200 papers ordered. It should be noted that the authors of this literature search have been involved in related work for which relevant articles had already been acquired and reviewed. In addition to an electronic search of databases, the 
authors performed a manual search of the current literature for nearly all of 1991 and 1992 in the publications listed here:

- Water Resources Research

- Transport in Porous Media

- ASME Journal of Heat Transfer

- International Journal of Heat and Mass Transfer

- Journal of Geophysical Research

- International Journal of Rock Mechanics and Mining Sciences and Geomechanics Abstracts

- International Journal for Numerical and Analytical Methods in Geomechanics

- International Journal for Numerical Methods in Engineering

- International Journal of Multiphase Flow

- International Journal of Numerical Methods in Fluids

- Journal of Fluid Mechanics

- Applied Geochemistry

- Geochimica et Cosmochimica Acta

- American Journal of Science

\subsection{STRUCTURE OF REPORT}

This report has five main sections:

- Section 2 introduces the one-way coupling mechanisms between individual processes

- Section 3 synthesizes the coupling mechanisms to discuss three-way-coupled processes

- Section 4 synthesizes the coupling mechanisms to discuss four-way-coupled processes

- Section 5 provides a summary and recommendations for the study of coupled effects

- Appendices A through D summarize the four individual processes

This report attempts to focus on the proposed repository at Yucca Mountain, Nevada. Throughout this report, summaries and conclusions are presented in order to gauge the importance of a coupling mechanism in light of other coupling mechanisms and uncertainties in the processes. The assessments are based on the current understanding of the processes and data uncertainties, and may have to be modified as new data and information emerge. 


\section{ONE-WAY COUPLING MECHANISMS}

In this section, the coupling mechanisms between the individual processes are discussed. For example, the mechanisms of vaporization, vapor flow, condensation, condensate flow in fractures, and thermallydriven buoyant flows are discussed as some of the $\mathrm{T} \Rightarrow \mathrm{H}$ one-way coupling mechanisms. Another example is that of the $\mathrm{C} \Rightarrow \mathrm{H}$ coupling which may be due to changes in fracture surface mineralogy, fracture healing, fluid flow due to chemical osmosis driving forces, and/or chemically-induced changes in hydrologic properties (e.g., viscosity, vapor pressure). In total, there are twelve possible one-way couplings for the four processes considered:

$$
\begin{aligned}
& \mathrm{T} \Rightarrow \mathrm{H}, \quad \mathrm{H} \Rightarrow \mathrm{T}, \quad \mathrm{T} \Rightarrow \mathrm{M}, \quad \mathrm{M} \Rightarrow \mathrm{T}, \quad \mathrm{T} \Rightarrow \mathrm{C}, \quad \mathrm{C} \Rightarrow \mathrm{T}, \\
& \mathrm{H} \Rightarrow \mathrm{M}, \quad \mathrm{M} \Rightarrow \mathrm{H}, \quad \mathrm{H} \Rightarrow \mathrm{C}, \quad \mathrm{C} \Rightarrow \mathrm{H}, \quad \mathrm{M} \Rightarrow \mathrm{C}, \quad \mathrm{C} \Rightarrow \mathrm{M} \text {. }
\end{aligned}
$$

Considering coupling mechanisms between pairs of processes provides a fundamental basis for multiple couplings. For example, the two-way $\mathrm{T} \Leftrightarrow \mathrm{H}$ coupling is considered as the combination of two one-way couplings ( $\mathrm{T} \Rightarrow \mathrm{H}$ and $\mathrm{H} \Rightarrow \mathrm{T}$ ). Park (1991) states that understanding the effects of frequently complex multi-discipline couplings requires the "decoupling" into individual processes and couplings, and this is the approach adopted herein. The synergistic effects of complex couplings can be understood through the combination of multiple one-way couplings. It is emphasized that, by considering one-way couplings, the complete scope of coupled THMC processes is covered systematically. All one-way couplings are discussed in this section. This information constitutes the "backbone" of the report from which conclusions and recommendations are derived in subsequent sections.

\section{$2.1 \quad$ THERMAL $\Rightarrow$ HYDROLOGIC}

In this section, the influence of thermal processes on hydrologic processes is discussed. It has been recognized in the literature that thermal-hydrologic processes are expected to be important at the proposed repository and need to be considered in repository design and evaluation of performance objectives (DOE, 1988).

Thermally-driven fluid flow and redistribution has received considerable investigation in the literature (e.g., Pollock, 1986; Tsang and Pruess, 1987; White and Altenhofen, 1989; Pruess et al., 1990a,b; Buscheck and Nitao, 1992; Ross, 1992). A number of laboratory experiments (e.g., Manteufel et al., 1992) and field experiments (e.g., Patrick et al., 1986; Zimmerman et al., 1986a; Ramirez et al., 1991a) have been conducted. A general consensus appears in the literature that the emplacement of heatgenerating waste will lead to increased temperatures and thermal gradients near the waste containers. In unsaturated rock, this is expected to result in a drying of the nearby rock due to vaporization of the water. The vapor will flow to cooler regions in the rock and therein condense. From calculations, it is apparent that at least half of the vapor will condense in regions above the containers (Pollock, 1986; Buscheck and Nitao, 1992). In field experiments, downward liquid flow (presumably condensate flow) near heated zones has been observed (Zimmerman et al., 1986a; Ramirez et al., 1991a). Condensate flow onto waste packages is considered important in predicting corrosion processes, container lifetimes, waste form dissolution processes, and radionuclide migration.

Thermal processes are primarily described by the temperature field, while hydrologic processes are primarily described by the liquid saturation and relative humidity fields. Hence, our interest is in 
understanding how a temperature field (and its associated temperature gradient) can affect the liquid saturation and relative humidity profiles in a porous, fractured medium.

Five areas of discussion are included in this section: (i) vaporization and condensation, (ii) nonisothermal enhancement of vapor diffusion, (iii) dripping in fractures, (iv) buoyancy-driven flow, and (v) computer codes.

\subsubsection{Vaporization and Condensation}

Heat generation from radioactive materials emplaced in a geologic repository is expected to lead to increased temperatures around the waste. The increased temperatures lead to increased vapor pressure of the in situ water. Because of increased temperature, the water in the liquid phase will not be in equilibrium with the water in the gas phase. Some of the liquid will be vaporized to increase the partial pressure of water in the gas phase and achieve thermodynamic equilibrium. The temperature and vapor pressure can be related through the Clapeyron equation (Wark, 1983, pg 487).

$$
P_{V}=P_{V, 0} \exp \left[\frac{h_{L G} M_{W}}{R_{I}}\left(\frac{1}{T_{0}}-\frac{1}{T}\right)\right]
$$

where

$$
\begin{array}{ll}
\mathrm{P}_{\mathrm{V}} & =\text { vapor pressure at temperature } \mathrm{T}_{\mathrm{o}}\left(\mathrm{N} / \mathrm{m}^{2}\right), \\
\mathrm{P}_{\mathrm{V}, \mathrm{o}} & =\text { reference vapor pressure }\left(\mathrm{N} / \mathrm{m}^{2}\right), \\
\mathrm{h}_{\mathrm{LG}} & =\text { heat of vaporization }(\mathrm{kJ} / \mathrm{kg}), \\
\mathrm{M}_{\mathrm{W}} & =\text { molecular weight of water }(18.02 \mathrm{~g} / \mathrm{mole}), \\
\mathrm{R}_{\mathrm{I}} & =\text { ideal gas constant }[8.314 \mathrm{~J} /(\mathrm{mole}-\mathrm{K})], \text { and } \\
\mathrm{T}_{\mathrm{o}} & =\text { reference temperature }(\mathrm{K}) .
\end{array}
$$

As the porous medium dries, the amount of water in both the liquid and the gas phase decreases. Assuming thermodynamic equilibrium, the amount of water in the gas phase can be related to the potential energy of water in the porous medium. At equilibrium, the potential energy is equal for the water in both the gas and liquid phases. Two physical mechanisms are responsible for holding the water in the porous medium: capillarity and adsorption. As a general rule, capillarity is important at high saturations and adsorption is important at low saturations. The more prominent distinction between capillarity and adsorption is the potential energy measured through the capillary/adsorption pressure, $\mathrm{P}_{\text {CAPIADs }}$. The term $\mathrm{P}_{\text {CAP/ADS }}$ is also known as the capillary pressure, suction pressure, and matrix potential, among other terms. A relation known as Kelvin's equation (Thomson, 1871) is commonly used to predict the decrease in the partial pressure of water over a porous medium.

$$
P_{W, G}=P_{V} \exp \left(\frac{-M_{W} P_{C A P / A D S}}{\rho_{L} R_{I} T}\right)
$$

where

$$
\begin{array}{ll}
P_{W, G} & =\text { partial pressure of water in the gas phase }\left(\mathrm{N} / \mathrm{m}^{2}\right) \text {, and } \\
\rho_{\mathrm{L}} & =\text { density of liquid }\left(\mathrm{kg} / \mathrm{m}^{3}\right) .
\end{array}
$$


The water in the porous medium and in the gas phase is normally assumed to be in local thermodynamic equilibrium because of the long times associated with processes at the repository. From Clapeyron's equation and Kelvin's equation, the partial pressure of water in the gas phase can be calculated to be a function of $\mathrm{T}$ and $\dot{\mathrm{P}}_{\mathrm{CAP} / \mathrm{ADS}}$. A nonuniform temperature field then results in a nonuniform vapor pressure field. Air is expected to be present at the repository; hence, the gas phase will consist of two components: water vapor and air. The water vapor is expected to travel from hotter regions to cooler regions due to advection and/or diffusion. The advection mechanism will result from a gradient in the total gas pressure (Darcy's Law) and the diffusion will result from a gradient in partial pressure of water vapor (Fick's Law). Once the water vapor reaches cooler regions, it will condense. In summary, liquid water is expected to vaporize in regions of higher temperature, flow to regions of lower temperature, and condense.

The geologic repository is commonly approximated as a heat-generating, porous disk in an axisymmetric model, as in Figure $2-1$, or as a thin source in a two-dimensional (2D), cartesian crosssectional model, as in Figure 2-2. In many analyses, the repository is sufficiently thin compared to geologic length scales to neglect its width. Pollock (1986) performed thermal-hydrologic calculations using the model in Figure 2-2. Results of his calculations indicated a region of increased liquid saturation forming both above and below the repository horizon (see Figure 2-3). Although these calculations are outdated, more recent calculations yield the same trend (Buscheck and Nitao, 1992). The increase in liquid saturation above and below the repository is due primarily to vaporization of water near the heatgenerating repository, temperature-gradient driven flow of the water vapor, and condensation in cooler regions.

Buscheck and Nitao (1988, 1992), and Ramspott (1991) among others, have described a "constructive" use of heat where the heat generated by radioactive decay may lead to increased (i) water vaporization, (ii) vapor flow away from the waste packages, (iii) condensation in cooler regions, and (iv) liquid imbibition into the matrix. This sequence may keep the waste packages dry for thousands of years for a high repository heat loading (due to dense package spacing). Optimally, the condensed water will imbibe into the rock or be shed to the sides of the waste packages and flow downward by gravity drainage in the fractures. The processes of condensate drainage in fractures have recently attracted much attention, since it is expected that drainage may occur over considerable distances before being totally imbibed by the matrix. In this work, condensate drainage is expected to result in dripping in fractures, and is discussed in Section 2.1.3.

\subsubsection{Nonisothermal Enhancement of Vapor Diffusion}

Molecular diffusion of water vapor in the fractured, porous medium can be an important transport mechanism, and can be strongly influenced by the temperature field. Philip and de Vries (1957), Pollock (1982), Mulay (1988), and others, have noted that a straightforward application of Fick's law underestimates the molecular diffusion of water vapor in a nonisothermal porous medium. This conclusion is based on comparison with experimental data, which have been found to be two to eight times larger than predictions based on Fick's law (Philip and de Vries, 1957). In a dry porous medium, a straightforward application of Fick's law appears valid. In a wet porous medium, the effect of liquid-vapor interaction may be important in the overall rate of vapor transfer. Philip and de Vries (1957) proposed that Fick's law be multiplied with an empirical nonisothermal enhancement factor.

$$
\overrightarrow{\mathrm{j}}_{\mathrm{W}, \mathrm{G}}=-\eta \tau \phi \mathrm{S}_{\mathrm{G}} \rho_{\mathrm{G}} \mathrm{D}_{\mathrm{WA}, \mathrm{G}} \nabla \mathrm{m}_{\mathrm{W}, \mathrm{G}}
$$




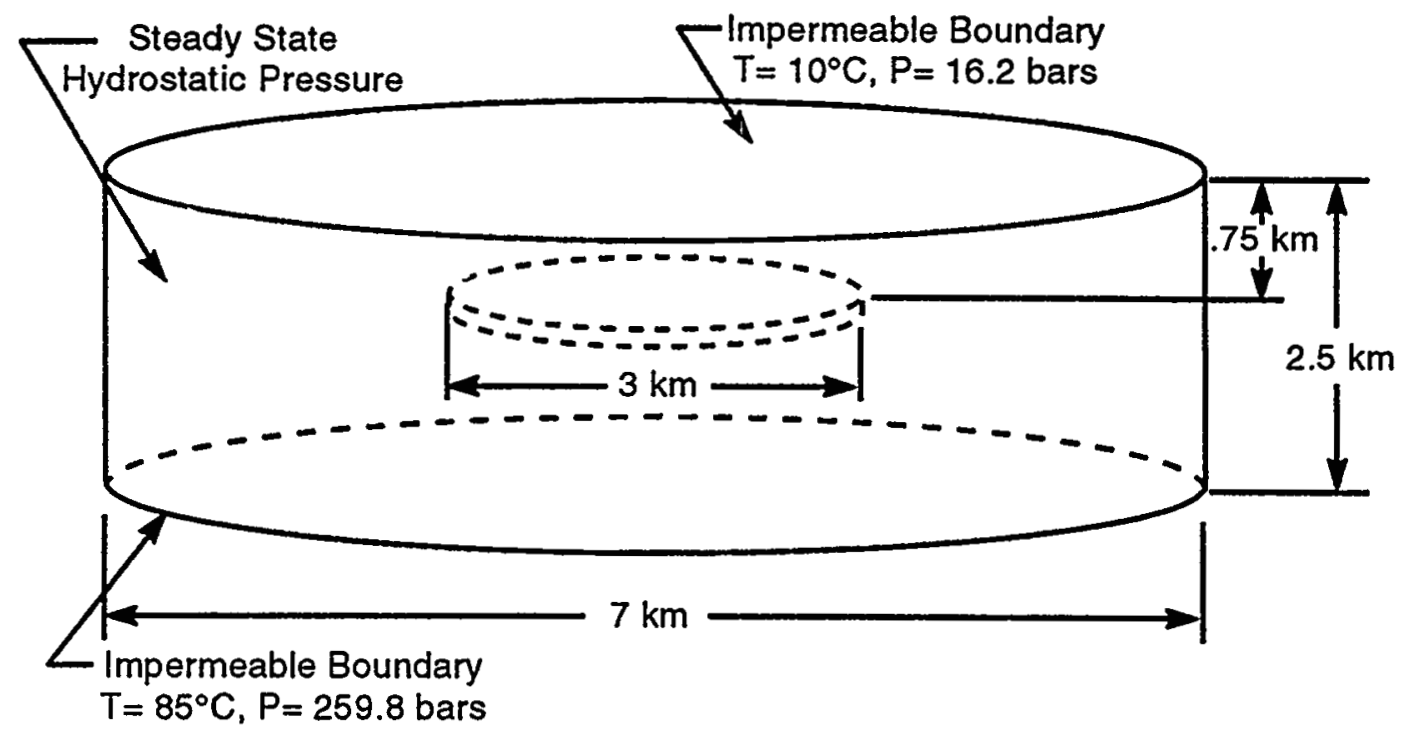

Figure 2-1. An axisymmetric model of a geologic repository [K. Verma and K. Pruess, Journal of Geophysical Research, 93(B2), 1159-1173, 1988, Copyright by the American Geophysical Union].

\section{Land Surface}

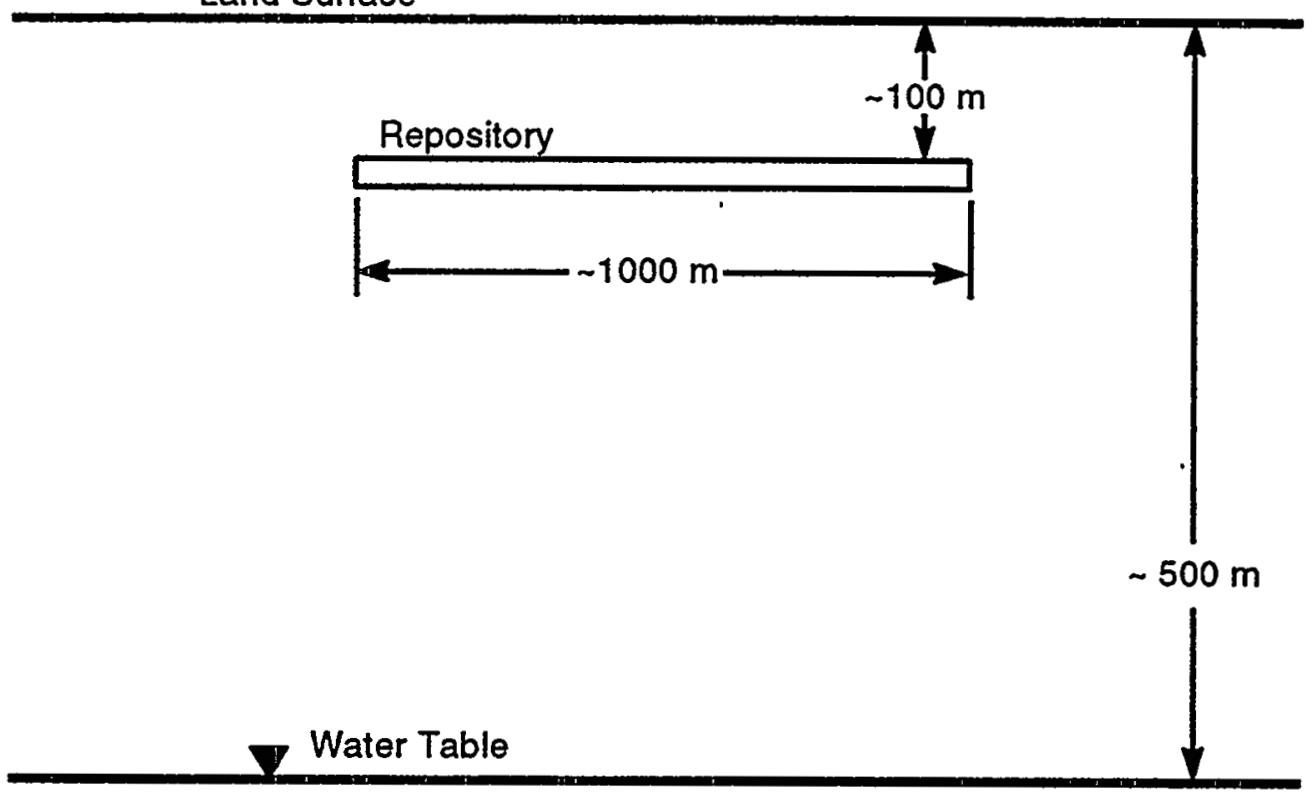

Figure 2-2. A 2D, cartesian model of a geologic repository [D.W. Pollock, Water Resources Research, 22(5), 765-775, 1986, Copyright by the American Geophysical Union]. 

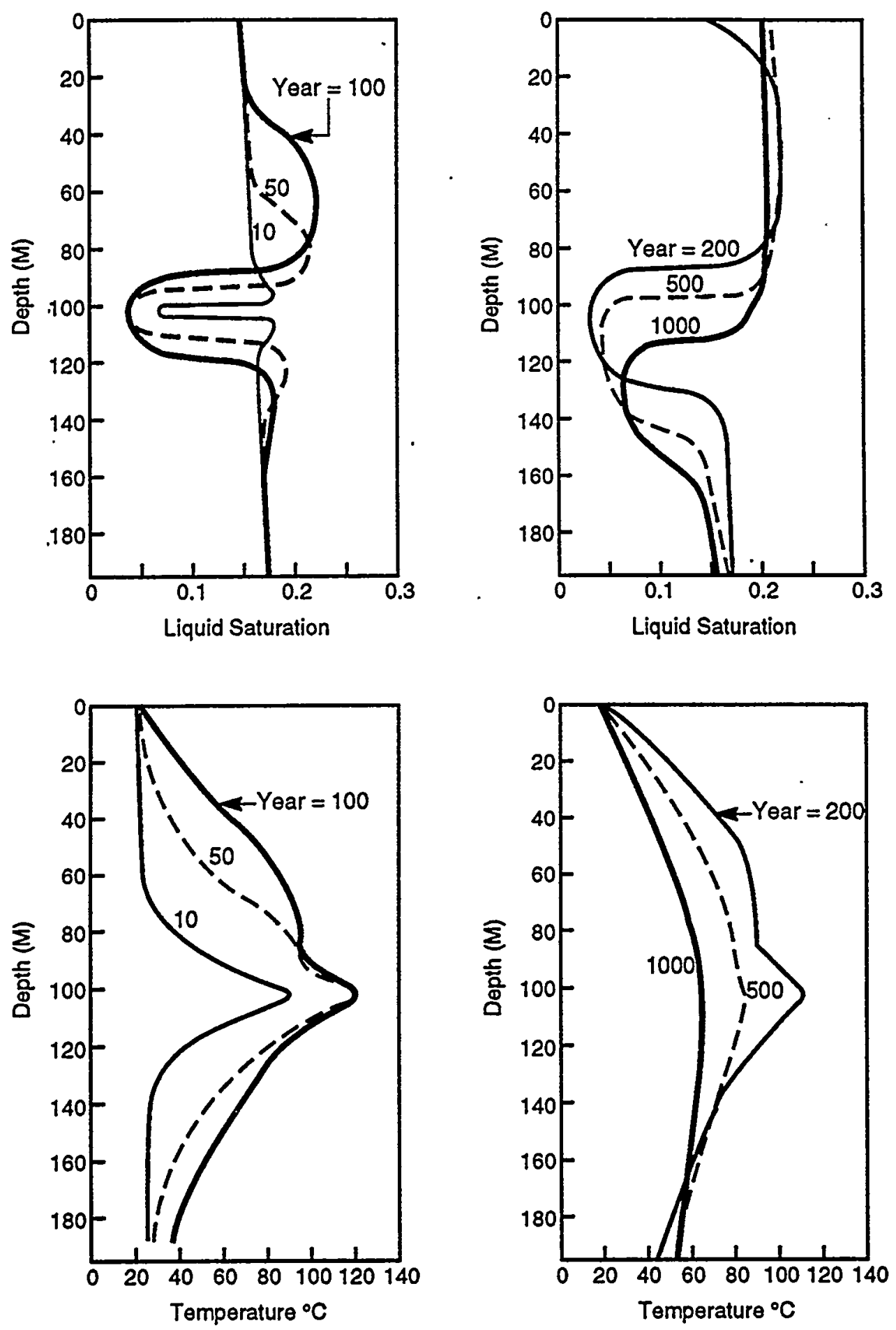

Figure 2-3. Liquid saturation and temperature distributions through the center of a repository from ground surface (depth $=0 \mathrm{~m})$ to water table $($ depth $=500 \mathrm{~m})$ at time $=(10,50,100,200,500,1000)$ years, see Figure 2-2 [D.W. Pollock, Water Resources Research, 22(5), 765-775, 1986, Copyright by the American Geophysical Union]. 
where

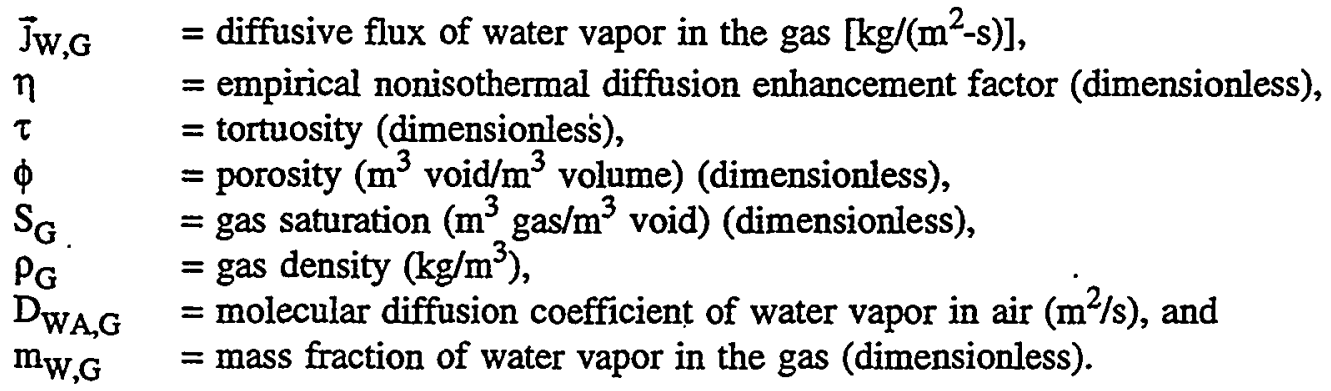

Two explanations for nonisothermal enhancement are: (i) coupled gas-liquid transfer, and (ii) increased temperature gradients in the gas phase. The explanations are not discussed here; however, it is noted that not all researchers acknowledge the need for a nonisothermal enhancement factor. Pruess (1987) does not discuss nonisothermal vapor diffusion enhancement, but has adopted a straightforward application of Fick's law $(\eta=1)$. Similarly, Bixler (1985), Forsyth (1990), and Forsyth and Simpson (1991) do not discuss nonisothermal vapor diffusion enhancement. In comparison, Pollock (1982) and Mulay (1988) discussed the importance and necessity of the diffusion enhancement factor. Because of the lack of a solid theoretical basis (although it has been reported experimentally), the nonisothermal diffusion enhancement factor for water vapor is typically neglected.

\subsubsection{Dripping in Fractures}

There are two primary mechanisms whereby liquid water may come into contact with waste packages, potentially leading to accelerated corrosion and release of radionuclides: episodic (e.g., incidental series of events) infiltration from the ground surface, and condensate drainage. If liquid flows in fractures, then it may travel great distances in short periods of time. Episodic infiltration is primarily a hydrologic process and does not result from thermal processes; hence, it is discussed in Section B.3. Vapor flow, condensation, and dripping are thermally driven; hence, they are discussed here.

Buscheck and Nitao (1992) suggest a repository with a sufficiently large power density will result in significant rock dry-out in the near-field, and flow of water vapor (primarily through fractures) to cooler regions, where it condenses. If the matrix has a low permeability, condensate may drain considerable distances along fractures before being totally imbibed by the matrix. The locations of condensate dripping in fractures and the magnitude of liquid flow rates are unresolved issues.

A cross-section of an emplacement drift is shown in Figure 2-4. Here, the drift is assumed to be dome shape and the waste packages are assumed to be emplaced vertically. The surrounding rock is shown to be highly fractured. The waste package is heat-generating and is expected to create a dry zone, a condensation zone, and regions of condensate dripping, as shown in Figure 2-5. For low power densities, the amount of water redistributed in the medium may be small enough so that the condensation is imbibed in the matrix as fast as it is produced. At higher power densities, the rate of growth of the dry zone is faster, so that more condensate is created at an increased rate. It is conceivable that, under high power densities, condensate will drain in the fractured medium.

At short times after emplacement, the dry zone is small, hence the amount of liquid in the condensation zone is small (see Figure 2-5). At longer times, the dry zone increases in size, and, similarly, the amount of liquid in the condensation zone also increases. Current repository design proposals have 


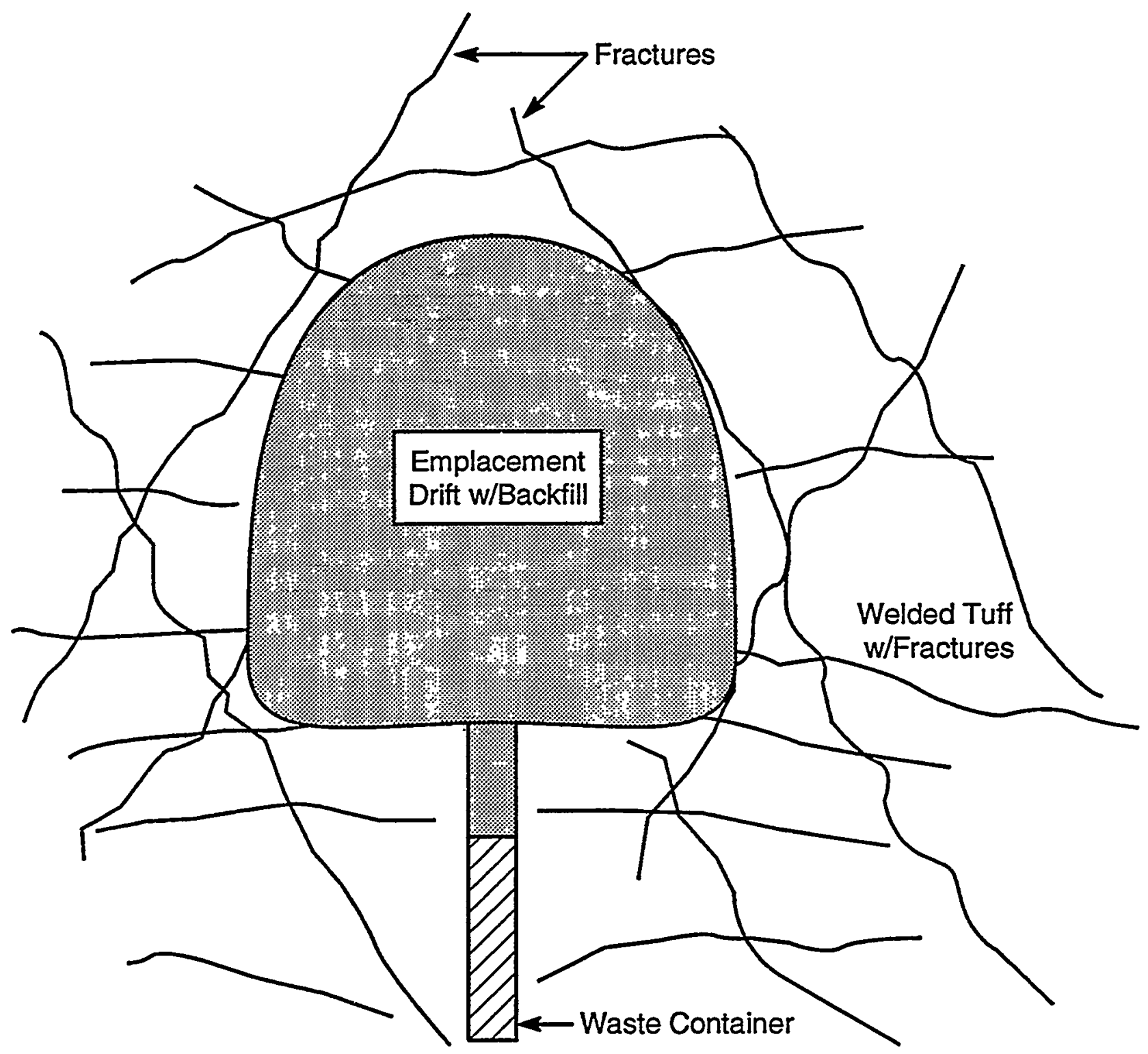

Figure 2-4. Ilustration of a drift with vertical emplacement of a waste container in a fractured porous medium. 

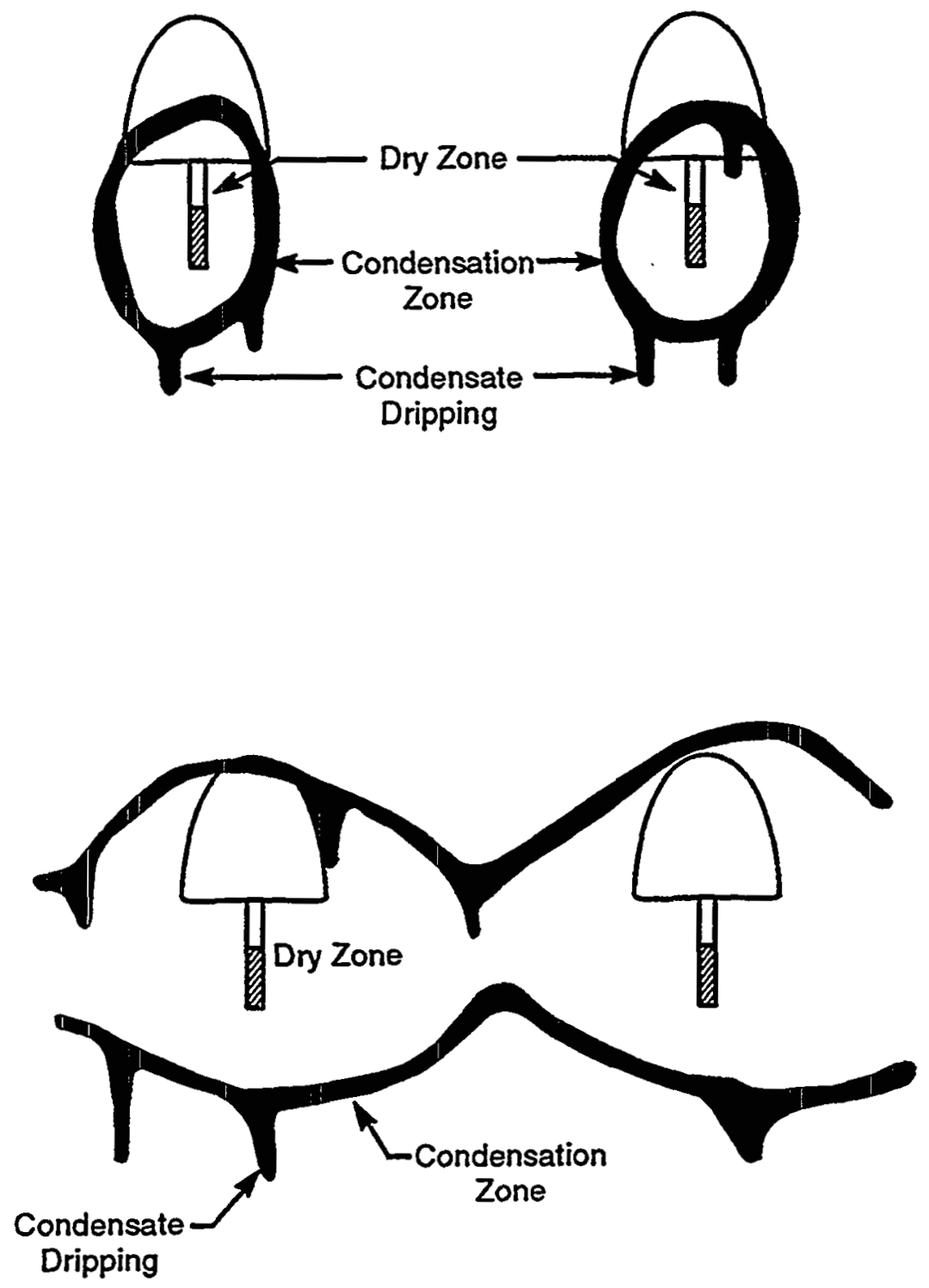

Figure 2-5. Illustration of a dry zone and a condensation zone with condensate dripping in fractures for short times (above) and longer times (below). 
parallel drifts. At long times, the condensate zones between emplacement drifts may interact, and probably encourage dripping between drifts, as shown in Figure 2-5. For a non-fractured, homogeneous, isotropic medium, the condensate above the waste packages would preferentially drain between drifts. However, fracture flow may be a dominant mechanism which would dictate where condensate drains.

It has been hypothesized that the mineralogy of the fracture surfaces may be important in resisting condensate imbibition into the matrix. Gallegos et al. (1992) investigated the effects of lowpermeability mineralized layers at the matrix/fracture interface, which may substantially reduce matrix imbibition and result in fracture-dominated flow. The mineralized layers on the fracture faces can inhibit the uptake of water into the adjacent matrix. This effect may invalidate the application of effective continuum models, which are based on matrix/fracture equilibrium and typically predict matrix-dominated flows except for values of liquid saturation near unity (this is discussed in Appendix B.3). Gallegos et al. (1992) conducted laboratory imbibition experiments and have reported that fracture coatings may severely reduce matrix uptake; however, these conclusions are based on only four tuff samples and only one of the four samples had a dramatic effect (reduction in imbibition by seven orders of magnitude). Imbibition will also be affected by the continuity and type of mineral coating. At Yucca Mountain, minerals such as zeolites may enhance imbibition and "most fractures are only partially sealed"' (DOE, 1988, pg. 4-13).

\subsubsection{Buoyancy-Driven Flow}

Many of the earlier studies related to geologic repositories involved saturated media. In a saturated medium, fluid motion is expected to accelerate the spread of radionuclides. The emplacement of heat-generating $\mathrm{HLW}$ is expected to elevate temperatures at the repository, which could create thermally-driven natural convection currents. Wang and Tsang (1980) studied buoyancy-driven flow at a hypothetical saturated repository (see Figure 2-6), which could increase fluid flow from the repository toward the ground surface and accelerate the transport of radionuclides.

In addition to a saturated medium, buoyancy-driven flow in the gas phase for a partially-saturated medium has been studied. Pollock (1986) modeled buoyant circulation in the gas phase at a hypothetical repository, as shown in Figure 2-7. In general, the elevated temperature at the repository results in decreased gas density and increased buoyancy of the lighter gas. Because Yucca Mountain is partially saturated, recent studies focus on gas phase buoyancy-driven flows (Amter and Ross, 1990).

Buoyancy-driven flow is especially important in modeling gas flow at Yucca Mountain, with respect to predicting gaseous transport of volatile and semi-volatile radionuclides such as ${ }^{14} \mathrm{C}$ in $\mathrm{CO}_{2},{ }^{129} \mathrm{I}$ in $\mathrm{I}_{2},{ }^{135} \mathrm{Cs}$ in $\mathrm{Cs}$, ${ }^{99} \mathrm{Se}$ in $\mathrm{SeO}_{2}$, and ${ }^{99} \mathrm{Tc}$ in $\mathrm{Tc}_{2} \mathrm{O}_{7}$. In early analyses, the release of ${ }^{14} \mathrm{C}$ has been shown to be near or exceeding regulatory limits (van Konynenburg, 1991; Codell and Murphy, 1992). The influence of temperature on the release and gaseous transport of radionuclides is considered important.

For a semi-infinite, saturated, porous medium, which is heated at the bottom and cooled at the top, there exists a critical Rayleigh number, $\mathrm{Ra}_{\mathrm{C}}$, below which the fluid remains stagnant and above which the fluid flows (Cheng, 1978; Bejan, 1984; among others). The traditional Rayleigh number is described as the ratio of buoyancy forces to viscous forces 


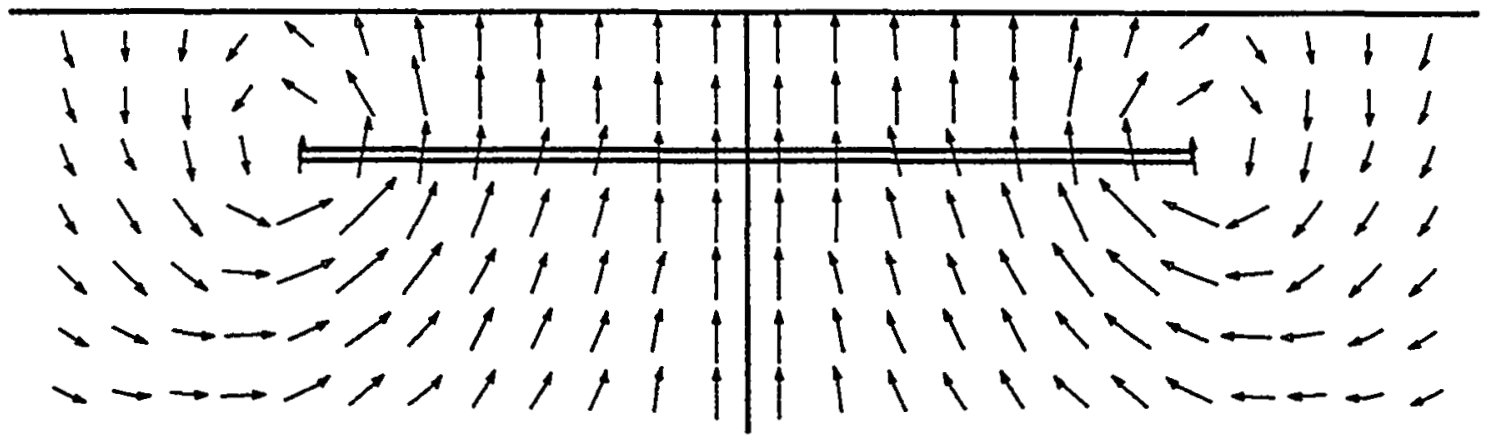

Figure 2-6. Buoyancy driven convection patterns around a saturated repository (Wang and Tsang, 1980).

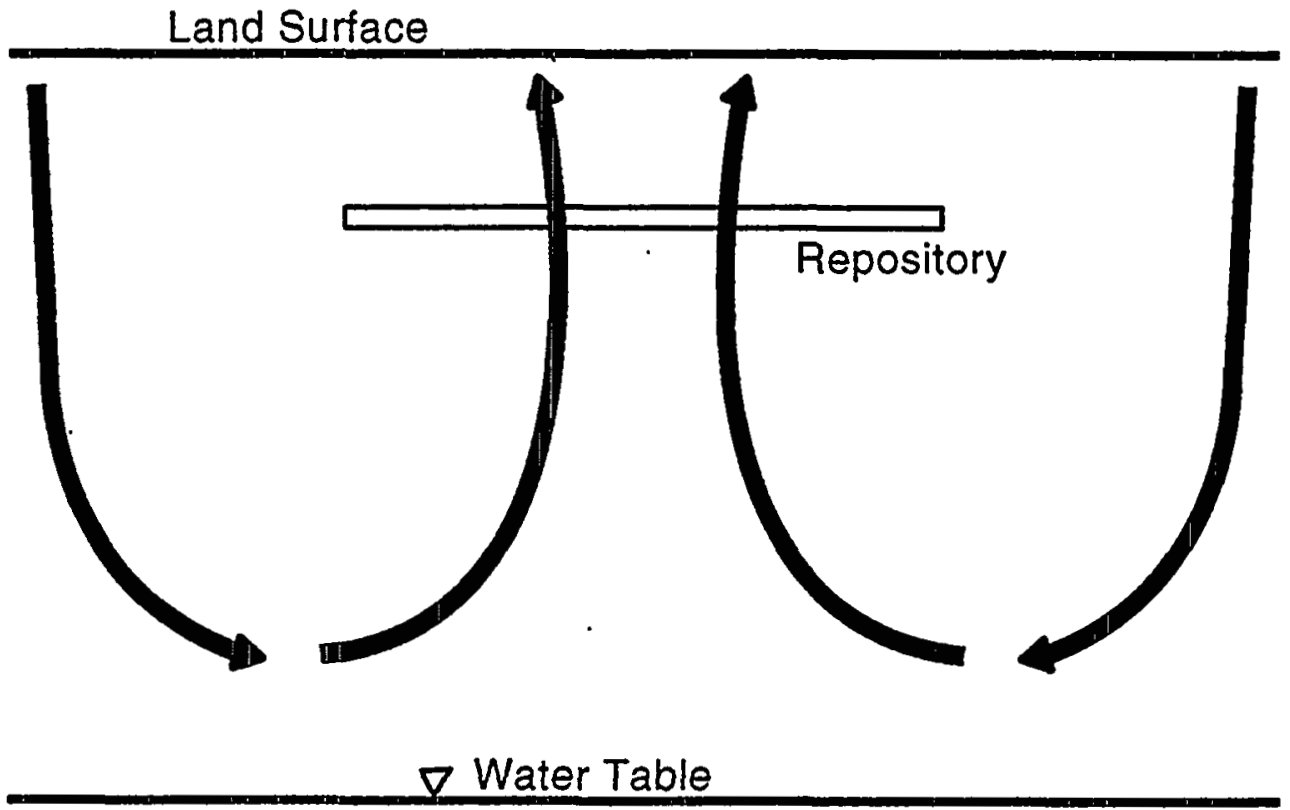

Figure 2-7. Illustration of convective circulation pattern for the gas phase [D.W. Pollock, Water Resources Research, 22(5), 765-775, 1986, Copyright by the American Geophysical Union]. 


$$
\mathrm{Ra}=\frac{\mathrm{g} \beta \Delta \mathrm{T} \mathrm{L}^{3}}{v \alpha}
$$

where

$$
\begin{array}{ll}
\mathrm{Ra} & =\text { Rayleigh number (dimensionless), } \\
\mathrm{g} & =\text { gravitational constant }\left(9.81 \mathrm{~m} / \mathrm{s}^{2}\right), \\
\beta & =\text { thermal expansion coefficient }\left(\mathrm{K}^{-1}\right), \\
\Delta \mathrm{T} & =\text { appropriate temperature difference }(\mathrm{K}), \\
\mathrm{L} & =\text { appropriate macroscopic length scale }(\mathrm{L}), \\
v & =\text { kinematic viscosity }\left(\mathrm{m}^{2} / \mathrm{s}\right), \text { and } \\
\alpha & =\text { thermal diffusivity }\left(\mathrm{m}^{2} / \mathrm{s}\right) .
\end{array}
$$

The porous medium Rayleigh number is defined as the traditional Rayleigh number times the Darcy number (Bejan, 1984; among others).

$$
\mathrm{Ra} \cdot \mathrm{Da}=\frac{\mathrm{g} \beta \Delta \mathrm{T} \mathrm{k}_{\mathrm{SAT}} \mathrm{L}}{v \alpha}
$$

where

$$
\begin{aligned}
& \mathrm{k}_{\mathrm{SAT}}=\text { saturated permeability }\left(\mathrm{m}^{2}\right), \text { and } \\
& \mathrm{Da}=\text { Darcy number }\left(\mathrm{k}_{\mathrm{SAT}} / \mathrm{L}^{2}\right) \text { (dimensionless). }
\end{aligned}
$$

The stability criteria in a porous medium are consistent with the "Benard problem," which is a classic problem presented in natural convection books (Tritton, 1977; among others). The stability criteria are depicted in Figure 2-8 where, for a saturated porous medium, the critical Rayleigh number is shown to be a function of the Darcy number, $\mathrm{Da}$. As the permeability of the medium increases, the Darcy number increases, and the porous medium approaches an open medium (no stationary solid phase). The classical results are that, for a porous medium, $\mathrm{Ra}_{\mathrm{C}} \cdot \mathrm{Da}=4 \pi^{2}$, and, for an open medium, $\mathrm{Ra}_{\mathrm{C}}=1708$ (Cheng, 1978; among others). Although not directly applicable to a partially-saturated repository, these classical results provide a starting point with which to estimate the presence or absence of buoyancy-driven flow. Assuming representative values for the proposed repository $\left(\mathrm{L}=200 \mathrm{~m}, \Delta \mathrm{T}=100^{\circ} \mathrm{C}\right.$ ), and gas properties for air $\left(\beta=1 /(300 \mathrm{~K}), v=20 \times 10^{-6} \mathrm{~m}^{2} / \mathrm{s}, \alpha=30 \times 10^{-6} \mathrm{~m}^{2} / \mathrm{s}\right)$, one can estimate that the gas phase permeability needs to be greater than $10^{-11} \mathrm{~m}^{2}$ in order for buoyancy-driven flow to be important. This value for permeability is considered large and barely within the range of effective fracture permeability, which is estimated to be as large as $\sim 10^{-11} \mathrm{~m}^{2}$ (Peters et al., 1984; Peters and Klavetter, 1988; Chesnut, 1992; among others). Admittedly, this analysis is simple and not directly applicable to the repository, because the rock units associated with the repository do not represent impermeable boundaries. On the contrary, the repository is expected to be located in the highly-fractured Topopah Spring unit (see Appendix B.2), hence the bulk permeability may be relatively high near the repository. Nevertheless, this simple analysis suggests that buoyancy-driven flow is not necessarily as important for the proposed repository as it might be for other potential repositories that were considered in a saturated medium. For an unsaturated repository, the buoyancy-driven liquid flow appears negligible, while the buoyancy-driven gas flow appears more important (Amter and Ross, 1990). Gas flow may also be due to the topographic and/or barometric conditions of Yucca Mountain (Weeks, 1987; Thorstenson et al., 1989; Nilson et al., 1991a,b) which could lead to significant gas velocities. 


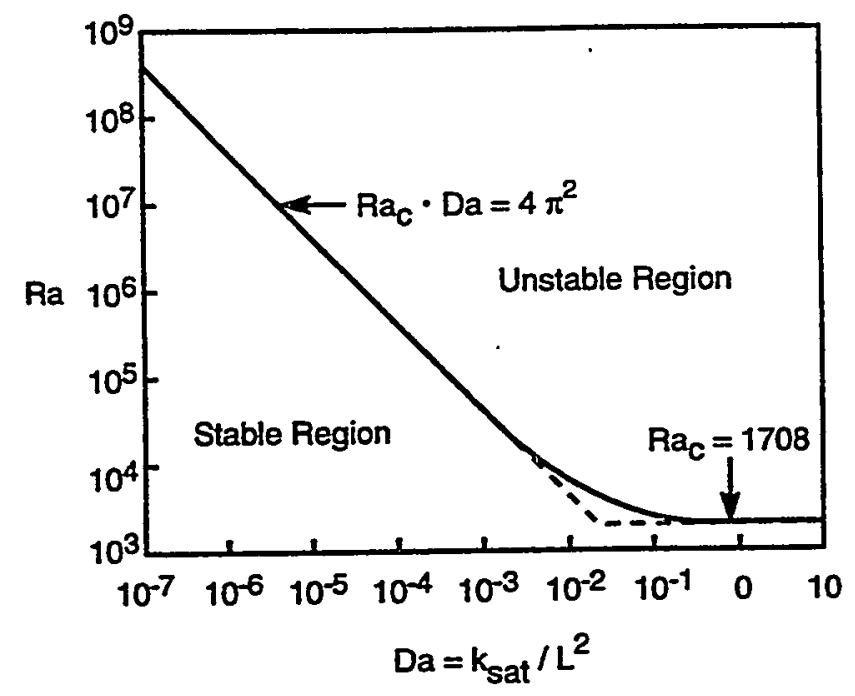

Figure 2-8. Critical Rayleigh number (for the Benard problem) versus dimensionless permeability from Darcy's limit to the viscous fluid limit.

Numerical analyses of gas flow at Yucca Mountain have been presented in the literature (e.g., Kipp, 1987; Amter and Ross, 1990; Lu et al., 1991). A common feature of these calculations is that the value of the effective gas permeability strongly influences the predictions (higher permeabilities result in higher gas velocities), yet the gas permeabilities at Yucca Mountain appear poorly characterized. Some results indicate that the gas permeability is relatively high, and may be as high as $4 \times 10^{-11} \mathrm{~m}^{2}$ (Chesnut, 1992). The estimates of gas permeability are typically described as being due to a relatively small number of connected pathways through large-aperture fractures (Chesnut, 1992). Gas flow path lines and travel times are highly dependent on the repository temperature as well as the degree of contrast between the effective permeabilities of welded and nonwelded units at Yucca Mountain (Lu et al., 1991). It is anticipated that site characterization activities will greatly improve the understanding of effective gas permeabilities at Yucca Mountain.

The relative importance of buoyancy-driven natural convection and molecular diffusion of water vapor was numerically compared by Tsang and Pruess (1987) (Figures 2-9 and 2-10). Tsang and Pruess performed two calculations using an axisymmetric model of the repository (similar to Figure 2-1). In Figure 2-9, molecular diffusion is considered where the gas flux near the repository (both above and below) is away from the repository. In Figure 2-10, molecular diffusion is neglected and the flow through the repository is much less and always directed above the repository. From these calculations, molecular diffusion is predicted to be important near the repository. At distances further from the repository, the gas flow is much smaller, yet has a characteristic flow pattern of buoyancy-driven natural convection. Tsang and Pruess (1987) estimate the gas phase Darcy velocity to be about $4 \mathrm{~cm} / \mathrm{yr}$ for a $95^{\circ} \mathrm{C}$ repository temperature with effective fracture permeability of $1.8 \times 10^{-14} \mathrm{~m}^{2}$. Tsang and Pruess (1987) consider this to be a "large" velocity; however, this appears to be quite small given that the repository is $\sim 200 \mathrm{~m}$ below ground surface. For "contaminant" transport, Tsang and Pruess (1987) note that the "pore" velocity is most appropriate. Using an effective fracture porosity of 0.0018 , the gas pore velocity is $22 \mathrm{~m} / \mathrm{yr}$, which is considerably larger than the Darcy velocity. Tsang and Pruess (1987) conclude that the simulated gas flow velocities depend on the effective gas permeabilities, fracture porosity, and tortuosity, which have not been well established at Yucca Mountain. Site characterization activities at Yucca Mountain are expected to allow a more definitive assessment of buoyancy-driven gas flows. 


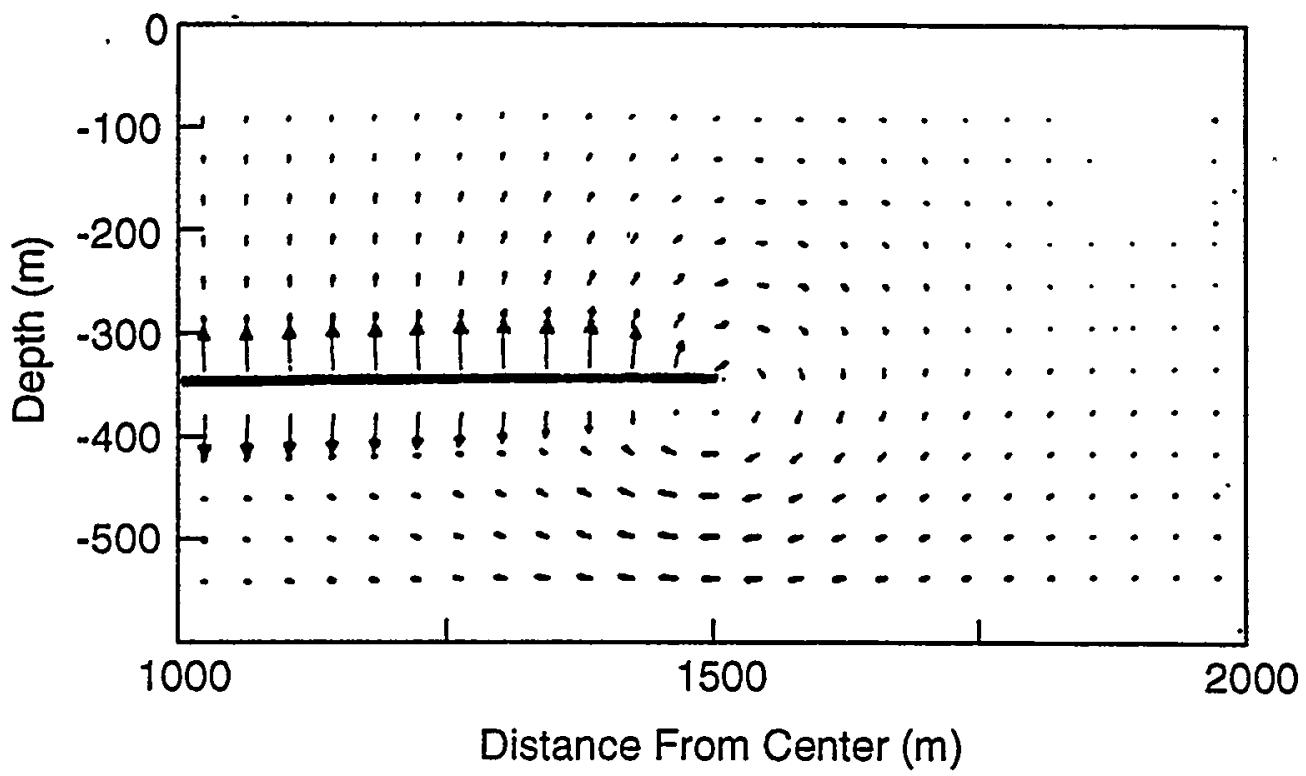

Figure 2-9. Gas flux at 100 yrs after waste emplacement showing flow away from repository primarily due to diffusion of water vapor [Y.W. Tsang and K. Pruess, Water Resources Research, 23(10), 1958-1966, 1987, Copyright by the American Geophysical Union].

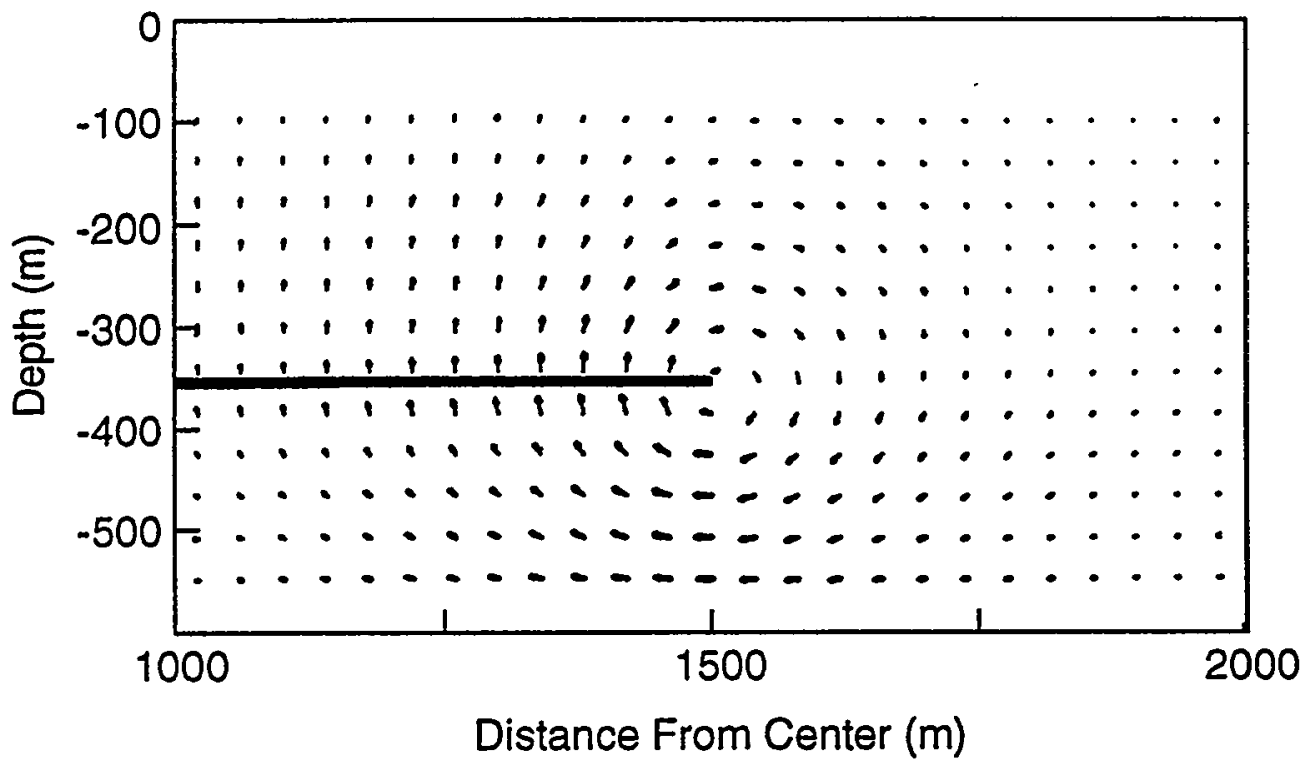

Figure 2-10. Gas flux at 100 yrs after waste emplacement neglecting molecular diffusion of water vapor [Y.W. Tsang and K. Pruess, Water Resources Research, 23(10), 1958-1966, 1987, Copyright by the American Geophysical Union]. 


\subsubsection{Computer Codes}

A number of computer programs have been written to predict thermal-hydrologic processes. Especially since 1985, there has been a dramatic increase in the numerical modeling of thermal-hydrologic processes in a partially-saturated porous medium.

\subsubsection{TOUGH}

Pruess (1987) has developed the TOUGH program which has received extensive usage (Pruess et al., 1990a,b; Doughty and Pruess, 1988; among others). TOUGH is derived from the MULKOM family of multiphase, multicomponent codes that were initially developed at Lawrence Berkeley Laboratory (LBL) for geothermal reservoir applications. It was developed for application to the proposed geologic HLW repository located in partially-saturated, fractured rock. TOUGH is capable of solving threedimensional (3D), two-phase (liquid and gas), two-component (water and air) fluid and heat transfer in a fractured, porous medium. The equations in TOUGH include gaseous diffusion, Darcy flow, capillary pressure, vaporization, and condensation. It is specifically designed for strongly heat-driven partiallysaturated flow, so that the heat of vaporization/condensation, conduction, and convection are included in the energy equation. The conservation equations include air, water (combined liquid and gas phase), and energy. The water in the gas phase is assumed to be in local thermodynamic equilibrium, so that the partial pressure of water in the gas equals the reduced vapor pressure through Kelvin's equation. TOUGH has been used at the CNWRA to simulate thermohydrologic laboratory experiments, however Green et al. (1992) reported numerical difficulties which limited the size of problems that could be simulated.

\subsubsection{VTOUGH}

Nitao (1989) has developed VTOUGH at Lawrence Livermore National Laboratory (LLNL) for DOE. VTOUGH is a modification of TOUGH with improved numerical convergence and stability properties. Both codes have been used at the CNWRA, where VTOUGH was found to be more robust (Green et al., 1992). The capabilities of these codes are comparable, and are discussed in Section 2.1.5.1.

\subsubsection{PORFLOW}

Runchal and Sagar (1992) developed PORFLOW to model fluid flow, heat transfer, and mass transport in variably-saturated geologic medium. The predecessor to PORFLOW (called PORFLO-3) was developed in support of environmental remediation activities being conducted at the Hanford Site for the DOE (Sagar and Runchal, 1990). A main feature of PORFLOW is its ability to model 3D geometries in both cartesian and cylindrical coordinate systems. The primary variables are hydraulic head (or pressure), temperature, and chemical species concentration (although the code only applies to non-reactive species). The most recent version of PORFLOW has the capability to model molecular diffusion, which is expected to be important near the waste containers.

\subsubsection{FEHMN}

Zyvoloski et al. (1991) have developed FEHMN to model heat and mass transfer using the finite element method. FEHMN was developed at Los Alamos National Laboratory (LANL) and was designed for the partially-saturated zone around the proposed Yucca Mountain repository. The code can solve 
nonisothermal, multiphase, multicomponent flow in a porous medium in cartesian 2D and 3D space, and in cylindrical coordinate systems.

\subsubsection{NORIA and PETROS}

Bixler (1985) developed NORIA, and Hadley (1985) developed PETROS, which are similar finite element codes for solving two-phase, two-component heat and mass transfer in porous media. Both codes were developed at Sandia National Laboratories (SNL) in support of the Nevada Nuclear Waste Storage Investigations (NNWSI). The major distinction is that NORIA is only applicable for 2D geometries, and PETROS is a one-dimensional (1D) code. Updegraff (1989) reported that NORIA was not able to simulate many test problems, including a radial boiling front problem, a heat pipe problem, a 2D infiltration problem, a convection cell problem, and a two-phase flow problem. Updegraff (1989) also reported that NORIA required significantly greater processing time than TOUGH for a number of test problems.

\subsubsection{FRAC-UNDX}

Clemo et al. (1990) developed FRAC-UNIX at EG\&G Idaho for the DOE. FRAC-UNIX is a 2D, saturated fluid and heat transport code for fractured porous media. FRAC-UNIX was originally designed to analyze fractured geothermal reservoirs; however, it has been updated to be used in support of license applications for HLW disposal. FRAC-UNIX uses a dual-permeability model for the matrix and fracture hydrology, and uses a random-walk (marker particle transport) model for heat transfer.

\subsubsection{GEOTHER}

Bian et al. (1987, 1988a,b) have developed GEOTHER (or GEO2) to model two-phase groundwater fluid flow and heat transport for high-level radioactive waste applications. GEOTHER evolved from a code developed by the U.S. Geological Survey (USGS). GEOTHER simulates multicomponent (i.e., water, noncondensable gases, rock/solid), porous and nonporous media (e.g., rock, waste container), two-phase (i.e., liquid, vapor) fluid, and heat transfer using the finite control volume method in cylindrical and 2D or 3D Cartesian coordinate systems.

\subsubsection{Summary and Conclusions}

In this section, the influence of the temperature field on hydrologic processes has been discussed. In particular, it has been noted that elevated temperatures will lead to vaporization of the groundwater. Temperature gradients will provide a driving force for water vapor flow into cooler regions. The water vapor will condense in cooler regions and potentially flow due to both gravity and gradients in capillary/adsorptive pressure. In a partially-saturated repository, vaporization, gas flow and condensation are anticipated to be important processes in affecting the hydrologic flow field.

The influence of a temperature gradient on vapor diffusion in a partially-saturated medium was discussed. In much of the earlier literature, differences were reported between experimental data and theoretical predictions based on a straightforward application of Fick's law. It has been hypothesized that water vapor and liquid in a nonisothermal porous medium interact to increase the effective vapor fluxes over theoretical predictions. The increase is most relevant in partially-saturated medium, and diminishes for either saturated or dry medium. However; the available literature appears to be inconclusive as to how this nonisothermal enhancement should be modeled, and it has been neglected in many recent studies. 
The importance of dripping in fracture networks was also discussed. Many of the hydrogeologic units at Yucca Mountain are known to be fractured with a low permeability matrix. Normally, fractures are expected to remain dry; however, episodic rainfall and infiltration at the ground surface, and water vapor condensation are two mechanisms whereby the fractures may have a substantial amount of liquid flow. Given a sufficient amount of liquid in an initially dry fracture, the liquid is expected to drip downward due to gravity forces. Thermal processes are expected to dry out the rock near the repository, and generate condensate dripping in fracture networks.

Computer programs that model coupled $\mathrm{T} \Rightarrow \mathrm{H}$ processes were also discussed. One major difficulty in mathematical models and computer programs is the characterization of matrix-fracture interactions. A significant number of programs have been developed which indicate that there is considerable interest in the scientific community in modeling these processes.

\section{$2.2 \quad$ HYDROLOGIC $\Rightarrow$ THERMAL}

Hydrologic processes (fluid flow) can influence thermal processes (temperature field). Coupled $\mathrm{H} \Rightarrow \mathrm{T}$ processes are important because they may cause (i) the formation of a heat pipe, (ii) condensate dripping, (iii) natural convection cooling, or (iv) changes in material properties such as saturationdependent thermal conductivity. In this chapter, all four areas are discussed.

\subsubsection{Heat Pipe}

A term "heat pipe" has been used in the engineering community to describe a situation where heat (thermal energy) is efficiently transferred with minimal temperature differences (Dunn and Reay, 1982). Heat pipes typically are engineered devices which consist of a sealed tube filled with a two-phase fluid. Frequently, the tube has an internal wicking region and a hollow region for the counter flow of liquid and vapor from one end of the tube to the other end. One end is subjected to a high temperature and the other to a lower temperature. Heat flows by vaporization of the fluid at the hot end, advection (transport due to bulk fluid motion) through the core to the cool end, where it condenses and is wicked back to the hot end. Hence, the fluid flow is circular and continuous, with vapor flowing from high to low temperature and liquid flowing from low to high temperature.

In many industrial applications, heat pipes are designed to increase the efficiency of thermal processes. Although less common, natural systems can develop "heat pipe" types of flow which transfer large amounts of heat (Schubert and Straus, 1979; Pruess, 1985; Turcotte, 1989; among others). For the proposed HLW repository, the potential impact of a natural heat pipe has been theoretically investigated by Doughty and Pruess $(1988,1990,1991,1992)$ and Pruess et al. (1990a,b). The heat-generating HLW creates a high-temperature source and the surrounding rock provides the low-temperature sink. The groundwater is vaporized near the HLW and flows through fractures to the lower temperature regions where it condenses. The condensed water may then flow back toward the HLW due to suction pressure gradients (frequently noted as capillary-driven flow) in the rock matrix.

Doughty and Pruess (1988) and Pruess et al. (1990a) have developed a model of the fluid and heat flow around a waste package as illustrated in Figure 2-11. After emplacement in the medium, the heat-generating waste is expected to dry a zone near the waste package. The heat-transfer mechanism in 


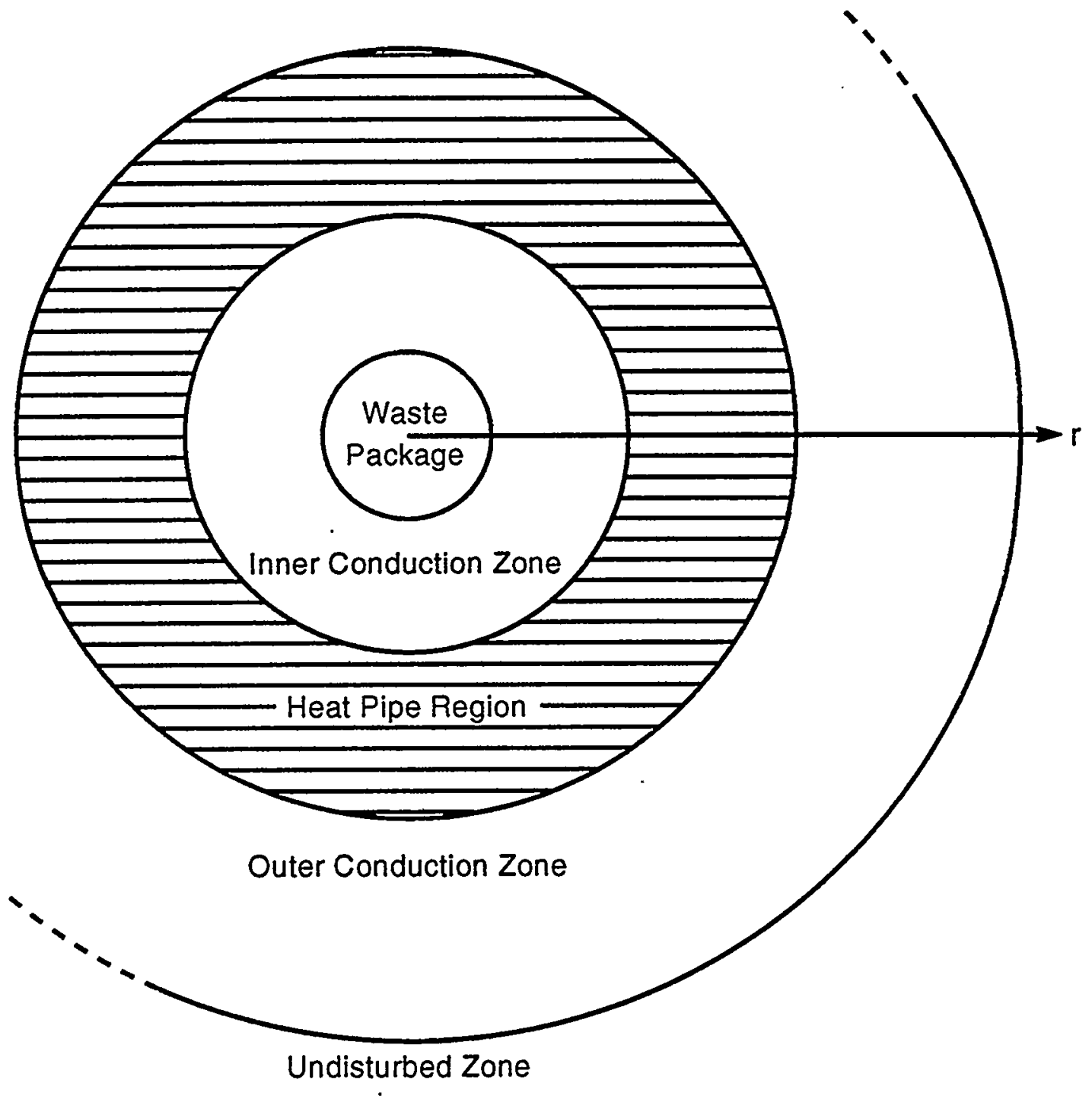

Figure 2-11. Schematic of heat-transfer regimes in a plane perpendicular to the axis of the waste package (not to scale) [K. Pruess, J.S.Y. Wang, and Y.W. Tsang, Water Resources Research, 26(6), 1235-1248, 1990a, Copyright by the American Geophysical Union]. 
the dry zone is primarily conduction, as indicated in Figure 2-11. The next region is the heat pipe, and the outer region is the outer conduction zone. Only in the heat pipe region is fluid flow expected to significantly influence the heat transfer.

The computed temperature $(T)$, gas pressure $(\mathrm{P})$, liquid saturation (S), and mole fraction of air in the gas phase (Y) are shown in Figure 2-12 for six different times, as computed by Doughty and Pruess (1988), using both a semianalytical solution and the TOUGH computer program (Pruess, 1987) for a continuum model of the medium. The heat pipe is distinguished by the region of near-constant temperature of $100^{\circ} \mathrm{C}$ (a temperature "plateau"). The development of the heat pipe is especially evident after 2 months. The inner conduction zone is distinguished by the increase in temperature above $100^{\circ} \mathrm{C}$, and can be seen after 1.3 yrs. The inner conduction zone continues to increase in size, which indicates that the water is continuously driven from the medium adjacent to the HLW package.

Pruess et al. (1990b) reported a comparison between an explicit fracture model and an effective continuum model for a heat pipe in a matrix whose permeability is representative of Topopah Spring densely welded tuff ( 2 microdarcy $=2 \times 10^{-18} \mathrm{~m}^{2}$, which is consistent with the values reported in Table B-1). The heat pipe is suppressed using the lower permeability matrix for either explicit fracture model (see Appendix B.3) or continuum model, as illustrated by the lack of a temperature plateau in Figure 2-13. The size of a heat pipe is strongly influenced by the matrix permeability, where high permeability encourages the development of a heat pipe (e.g., Figure 2-12 where $\mathrm{k}_{\mathrm{SAT}}-10^{-12} \mathrm{~m}^{2}$ ) and low permeability suppresses a heat pipe (e.g., Figure 2-13 where $\mathrm{k}_{\mathrm{SAT}} \sim 10^{-18} \mathrm{~m}^{2}$ ). Because of the low matrix permeability at the proposed repository, a heat pipe is not anticipated to be important (Buscheck and Nitao, 1992).

A number of assumptions are typically introduced in order to help accentuate the heat pipe effect including (i) gas pressure buildup at the waste package (by neglecting the air gap and connectivity between canister borehole and emplacement drift), (ii) liquid phase continuity for capillary pressures up to 100 kilobars, (iii) relatively high values of matrix permeability, and (iv) absence of non-condensibles (i.e., air) in the heat pipe region. In addition, experimental observations do not support the assumptions and conclusions reported by Doughty and Pruess $(1988,1990,1991,1992)$ and Pruess et al. $(1990 \mathrm{a}, \mathrm{b})$. In comparison, two field experiments (Zimmerman et al., 1986a; Ramirez et al., 1991a) have reported observations which do not appear to be the result of heat pipe phenomena. Significant differences exist between the heat pipe predictions and experimental observation, including: The mechanism for liquid flow (capillary-driven or gravity-driven), the location for liquid flow (matrix or fracture), the direction of liquid flow (towards heat source or decreasing elevation), and effect on the temperature of the heat source (strong or negligible). In the heat pipe, the liquid flow is predicted to be capillary-driven flow in the rock matrix towards the heat source, whereas in the experimental observations it has been gravity-driven flow in fractures in the direction of decreasing elevation. In heat pipe simulations, the influence of gravity is neglected whereas gravity, in addition to the location, orientation and connectivity of fractures, has been observed to be very important in field experiments. Lastly, the heat pipe simulations show a predictable cooling effect on the heat source where it has been reported that "Under [heat pipe] conditions ... the emplacement hole and formation temperatures will peak near $100^{\circ} \mathrm{C}$ " (Pruess et al., 1990a). Experimental observations, however, show the heat source temperature may have either a strong or negligible effect, depending on the location, orientation and connectivity of fractures for liquid condensate to drain onto the heat source. In particular, Zimmerman et al. (1986a) report one of three heater tests to have a strong effect on the temperature of the heat source, while Ramirez et al. (1991a) report negligible effects on the heater temperature. In summary, the available data strongly suggests that a heat pipe (or multiple heat pipes) will not be present at the proposed repository. 
$6 I-\tau$
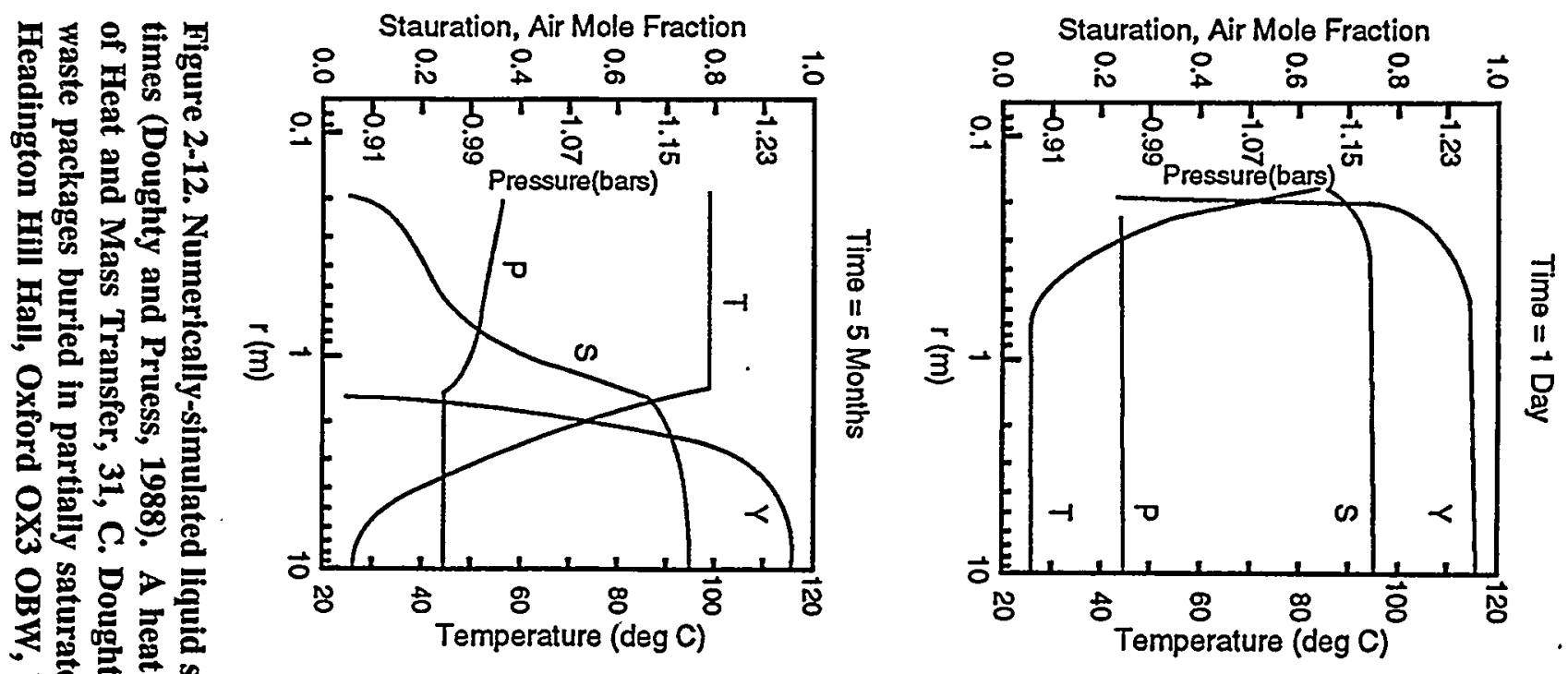

성 Stauration, Air Mole Fraction
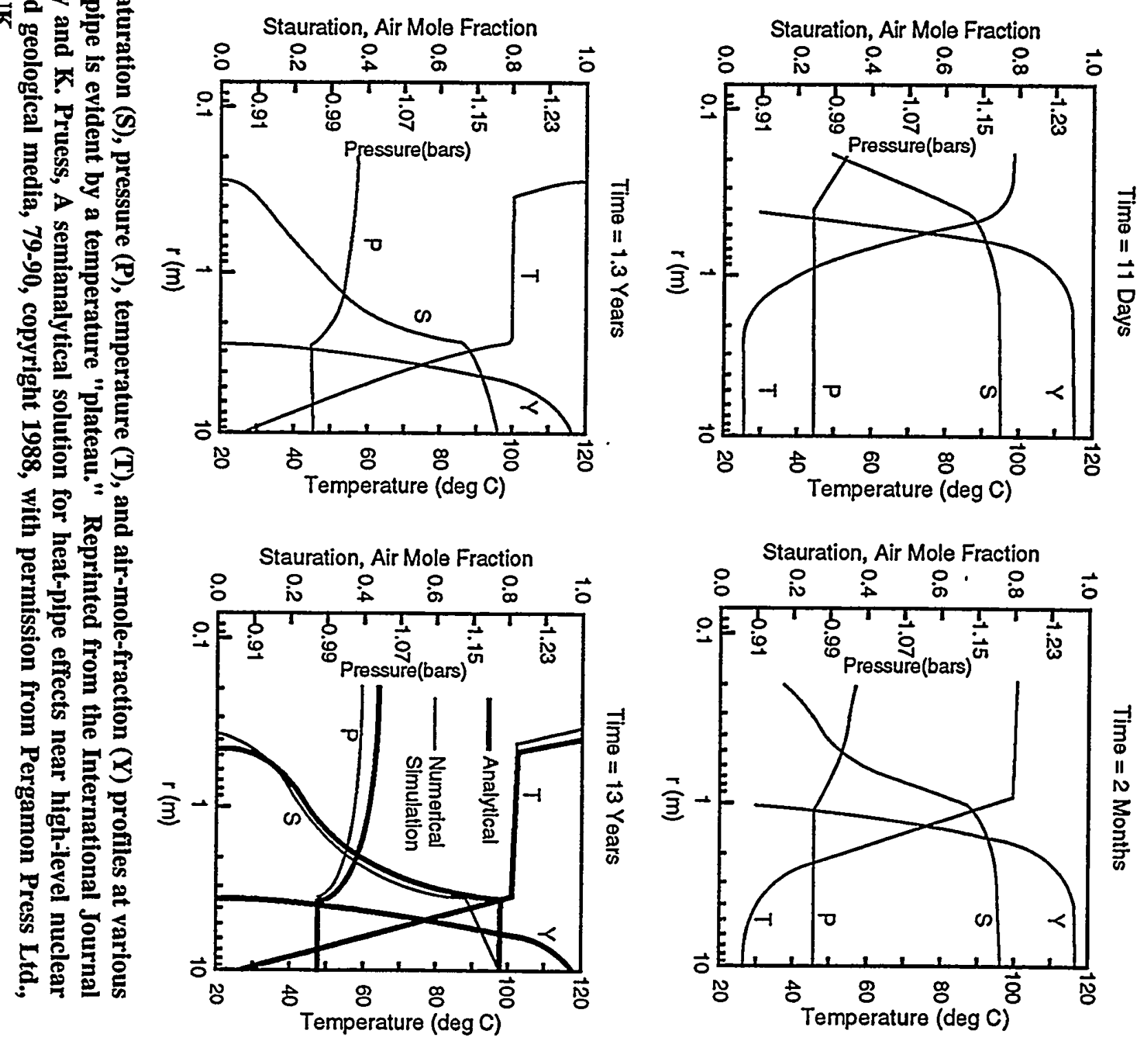


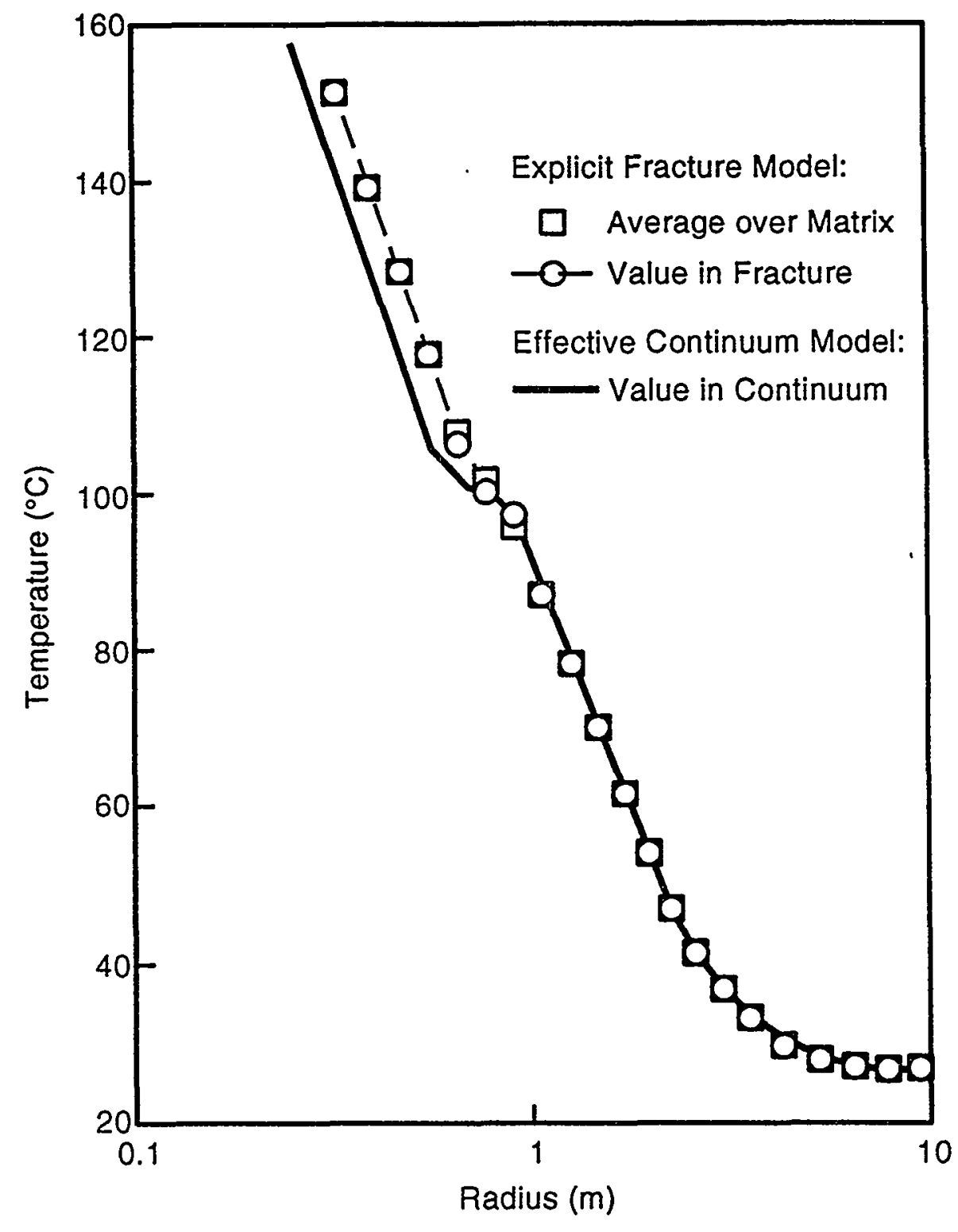

Figure 2-13. Temperature profiles showing a lack of a plateau near $100^{\circ} \mathrm{C}$, hence the absence of a heat pipe. Calculations based on an explicit fracture model and an effective continuum model using hydrologic properties representative of Topopah Spring densely welded tuff [K. Pruess, J.S.Y. Wang, and Y.W. Tsang, Water Resources Research, 26(6), 1249-1261, 1990b, Copyright by the American Geophysical Union]. 


\subsubsection{Condensate Dripping}

In Section 2.1.3 of this report, thermal processes are shown to provide the impetus for condensate formation, which can lead to condensate drainage. In this section, the feedback of liquid dripping flow is discussed as it may influence thermal processes, that is, the temperature field. A strong hydrologic feedback to the temperature field may occur when condensate drains into a "boiling zone," thereby maintaining the temperature near $100^{\circ} \mathrm{C}$. The heat will be transferred by conduction into the boiling region, where it is removed by vaporization and vapor flow.

Condensate dripping in fractures has been experimentally observed at the DOE-sponsored G-tunnel (located in the Rainier Mesa area of the Nevada Test Site) tests conducted by SNL (Zimmerman et al., 1986a) and by LLNL (Ramirez et al., 1991). The LLNL G-tunnel tests will be discussed first and the SNL G-tunnel tests will be discussed second.

In the LLNL G-tunnel tests, a heater was installed in a partially-saturated, welded, fractured tuff which has hydrologic properties similar to that at Yucca Mountain. The tests lasted on the order of months during which the rock near the heater dried and a "saturation halo" developed (Ramirez et al., 1991a; Buscheck and Nitao, 1992). It was observed that the in situ water vaporized near the heater and flowed primarily in fractures towards cooler regions, where it condensed. During the early times in the tests, the condensate was imbibed into the surrounding matrix. At later times, the condensate flowed in fracture networks due to gravity. The flow of water vapor was not influenced by gravity, while the condensate flow in the fractures was primarily gravity-driven. The condensate above the heater was observed to flow back towards the heater. The condensate below the heater flowed away from the heater. The combined vaporization, vapor flow, condensation, and gravity-driven dripping in fractures tended to shed moisture away from the heater. The possibility of condensate dripping onto waste containers at the proposed repository has been noted by Buscheck and Nitao (1992). Condensate dripping may be important in waste container corrosion processes, container integrity lifetimes, and waste form dissolution/mobilization processes.

The locations of temperature measurements and observed fractures for the LLNL G-tunnel tests are illustrated in Figure 2-14. The tests were conducted by drilling boreholes from two perpendicular drifts in the G-tunnel underground facility (rock mechanics drift and small diameter heater alcove). For clarity, only the heater borehole and the P2 and P3 instrumentation boreholes are shown in Figure 2-14. All boreholes were approximately horizontally oriented (with P3 having a downward slope). The heater borehole entered from the rock mechanics drift, and the P2 and P3 boreholes entered from the smalldiameter heater alcove.

A dry zone was observed around the heater using neutron logging measurements (Ramirez et al., 1991b). The fractures were experimentally observed to significantly affect the moisture redistribution and slightly affect the overall temperature field. The fractures provided a preferential pathway for vapor flow and condensate drainage. The condensate drainage then influenced the temperature field. Temperature measurements were taken along a line both above (line P2 in Figure 2-14) and below (line P3 in Figure 2-14) the heater, and are plotted in Figures $2-15$ and 2-16, respectively. A "plateau" was observed for some of the temperature measurements below the heater (see Figure 2-16, thermocouple numbers 88 and 89). These thermocouples were located to the lower left of the heater with 88 nearer the heater and adjacent to a vertically oriented fracture. It is noticeable that the plateau persisted for many days during the experiment. The value of the plateau is near $96^{\circ} \mathrm{C}$, which corresponds to the boiling temperature of 


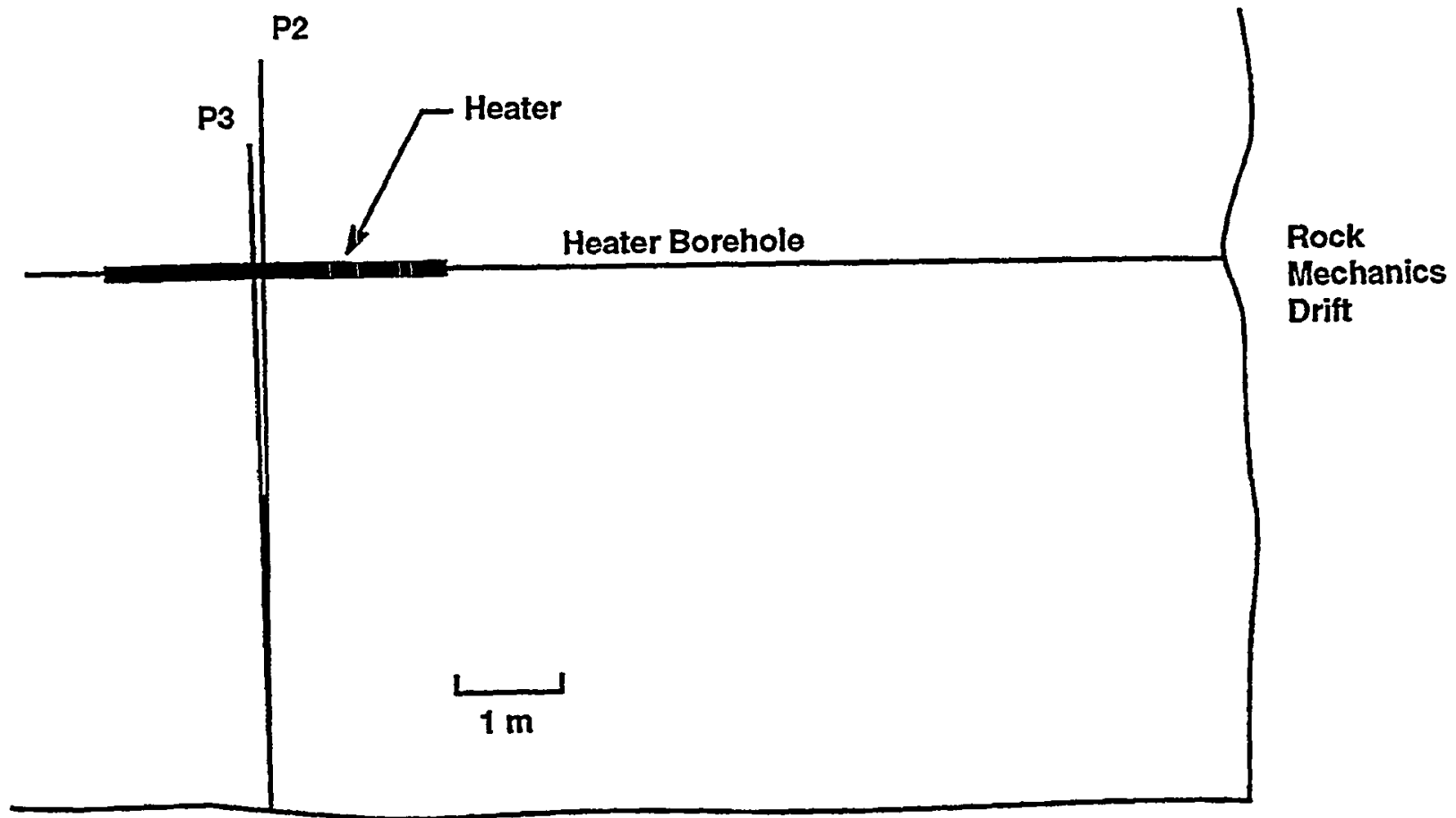

Small Diameter Heater Alcove

(a)

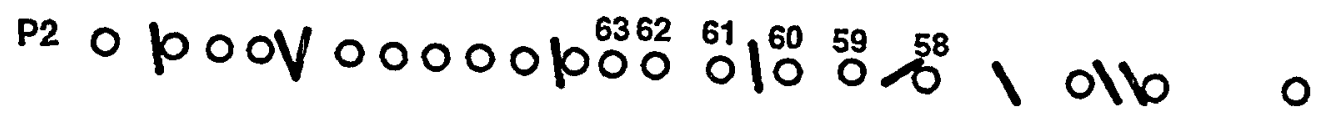

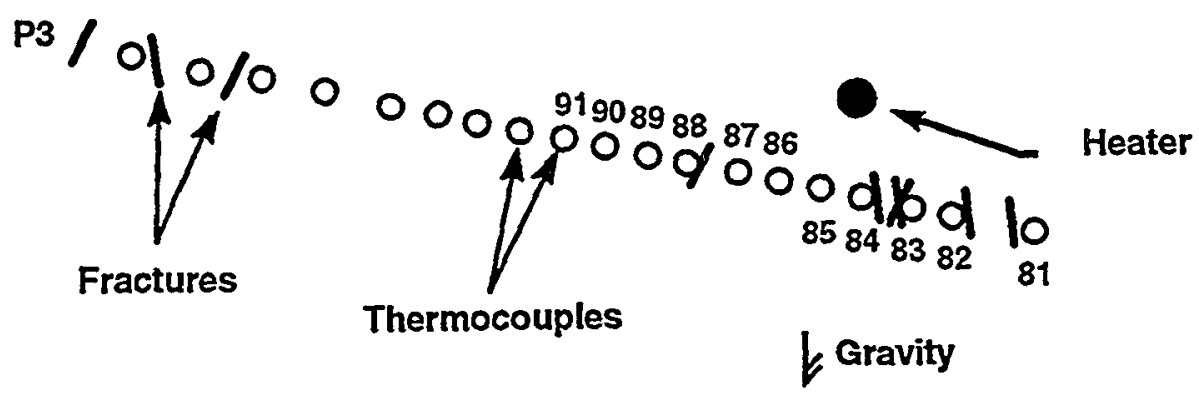

(b)

Figure 2-14. Plan view (a) and cross-sectional view (b) along boreholes P2 and P3 of the LLNL G-tunnel heater tests showing locations of thermocouples, fractures, and the heater (adapted from Ramirez et al., 1991a, pg 11 and pg 90). 


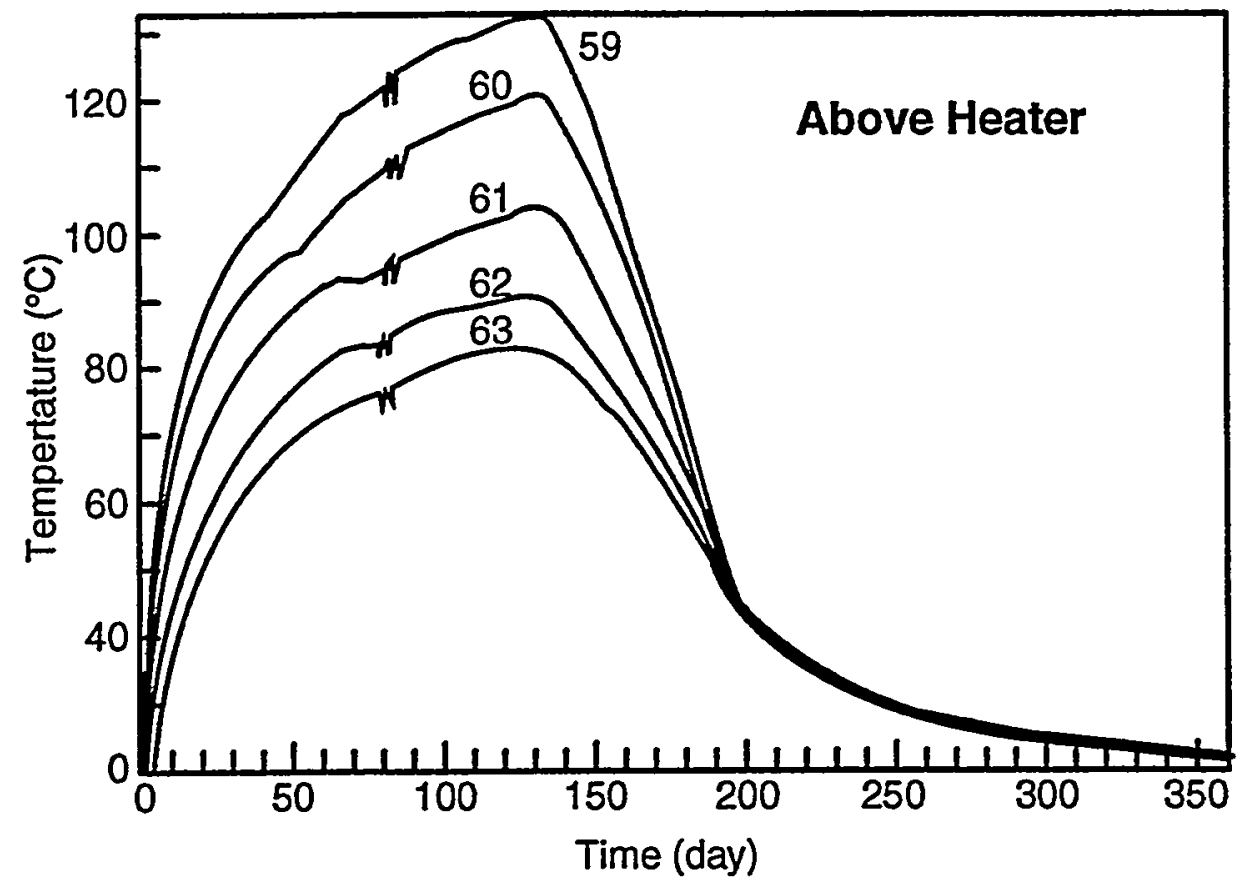

Figure 2-15. Temperature history of selected thermocouples above the heater (see Figure 2-14 for thermocouple locations) (adapted from Lin et al., 1991; also Ramirez et al., 1991a where an apparent error in the $\mathrm{x}$-axis scaling has been corrected).

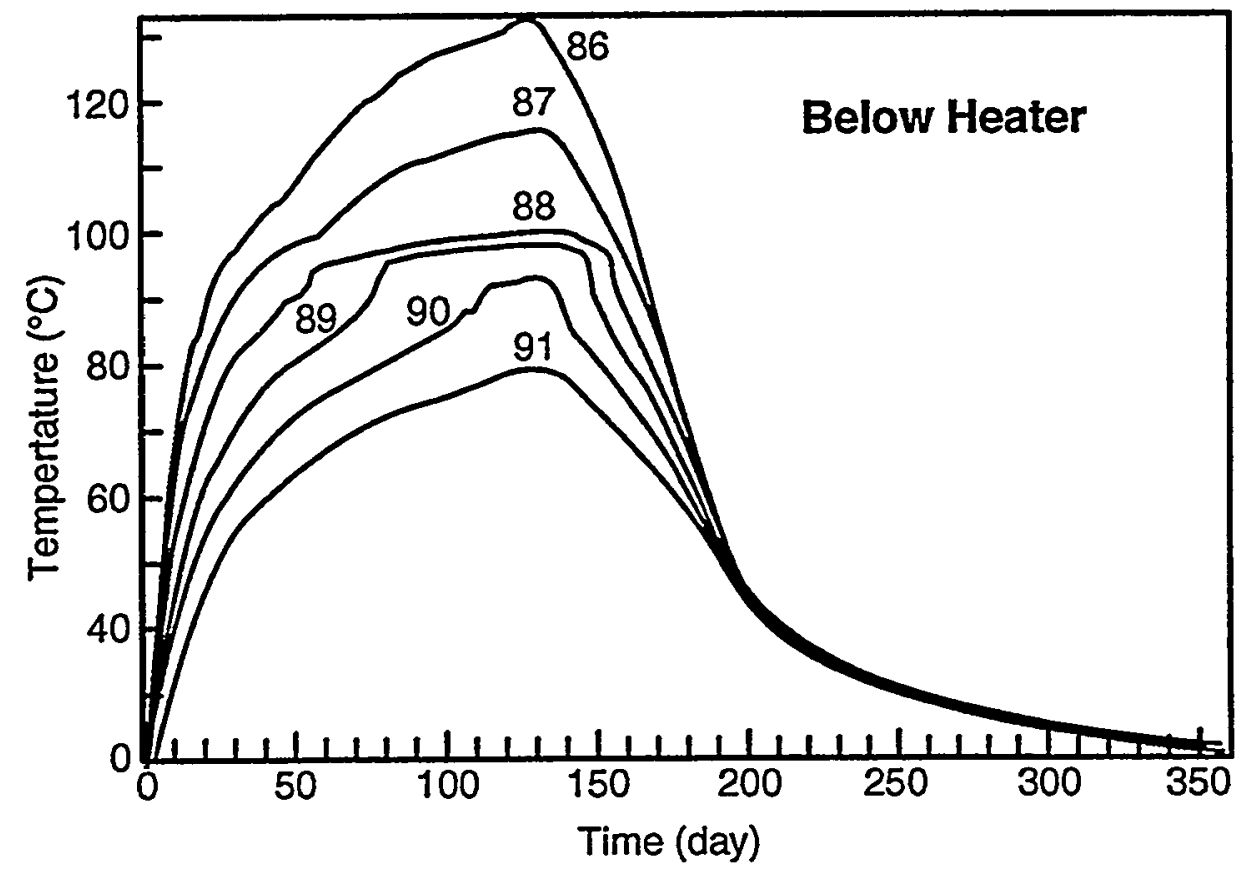

Figure 2-16. Temperature history of selected thermocouples below the heater (see Figure 2-14 for thermocouple locations) (Lin et al., 1991; also Ramirez et al., 1991a). 
water for the elevation of the G-tunnel experiments. Hence, it is believed that draining condensate in the fracture acted to supply fluid to the region where boiling occurred. None of the thermocouples above the heater experience a plateau; hence, gravity-driven condensate drainage is thought to be an important hydrologic process.

Zimmerman et al. (1986a) and Zimmerman and Blanford (1986) also reported an experimentally observed dry zone, boiling region, and condensation region in the SNL G-tunnel tests. The shape of the boiling region was governed by the presence (or absence) of fractures. Similarly, the boiling zone exhibited a temperature plateau of about $95^{\circ} \mathrm{C}$. "Water migrations" were noted to affect temperatures over localized regions of the rock, but these effects would disappear after the water was vaporized. Aside from localized regions in the rock, the hydrology did not significantly affect the temperature field. Zimmerman and Blanford (1986) attributed the observed temperature plateau to hydrothermal water migrations which were influenced by gravity and geological conditions (i.e., fractures). The localized nature of the plateau and the corroborating data from Ramirez et al. (1991a) and Lin et al. (1991) suggest that gravity-driven condensate drainage in fractures caused the temperature plateau (boiling region).

In another field experiment conducted at Climax (Patrick et al., 1986), temperatures were perturbed at locations where liquid flowed into a region of vaporization (i.e., boiling). This was evident in the "container emplacement hole, number 01" in the Climax tests, where recorded temperatures were equal to the local boiling point temperature, and water was observed to stand in the emplacement hole for extended periods of time (Patrick et al., 1986). It appears that a fracture allowed condensate (or downward flowing groundwater) to drain into the emplacement hole where it collected. The liquid flow appeared to be localized and had a negligible effect on the overall temperature field in the surrounding rock.

\subsubsection{Natural Convection}

In Section 2.1.4 of this report, the temperature field is noted to influence the hydrologic flow field due to buoyancy-driven natural convection. In this section, the hydrologic flow field is noted to be able to affect the temperature field. Buoyancy-driven natural convection pertinent to a saturated geologic repository has been studied by Kulacki and Keyhani (1987) and Rajen and Kulacki (1987), among others. The repository was assumed to be in a saturated, homogeneous, isotropic medium, and a repository-scale natural convection study was conducted. Rajen and Kulacki (1987) assumed the repository, as well as the side and top boundaries, to be impermeable. Although the boundary conditions and the assumption of a saturated homogeneous medium are not realistic for the present partially-saturated repository, these early studies do contain basic information with which to gauge the importance of the $\mathrm{H} \Rightarrow \mathrm{T}$ coupling.

The geometry used by Rajen and Kulacki (1987) is shown in Figure 2-17. The thermal conductivity of the porous medium was approximately $0.8 \mathrm{~W} /(\mathrm{m}-\mathrm{K})$, and the permeability was approximately $3.5 \times 10^{-9} \mathrm{~m}^{2}$. The boundary conditions are indicated in Figure $2-17$, where each boundary is impermeable to fluid flow. The central portion of the bottom was heated to a constant temperature $\left(T_{h}\right)$, while the remaining portions were thermally insulated. The sides were thermally insulated, and the top was cooled to a constant temperature $\left(T_{c}\right)$. The bottom temperature, $T_{h}$, was experimentally increased, thereby increasing the Rayleigh number and increasing buoyancy-driven natural convection. The experimental results (from a scaled laboratory setup) and numerical results are shown in Figure 2-18. The Rayleigh number is a measure of the strength of the thermal driving force to induce fluid motion. The Nusselt number is defined as the ratio of conductive plus convective heat transfer, divided by the stagnant 


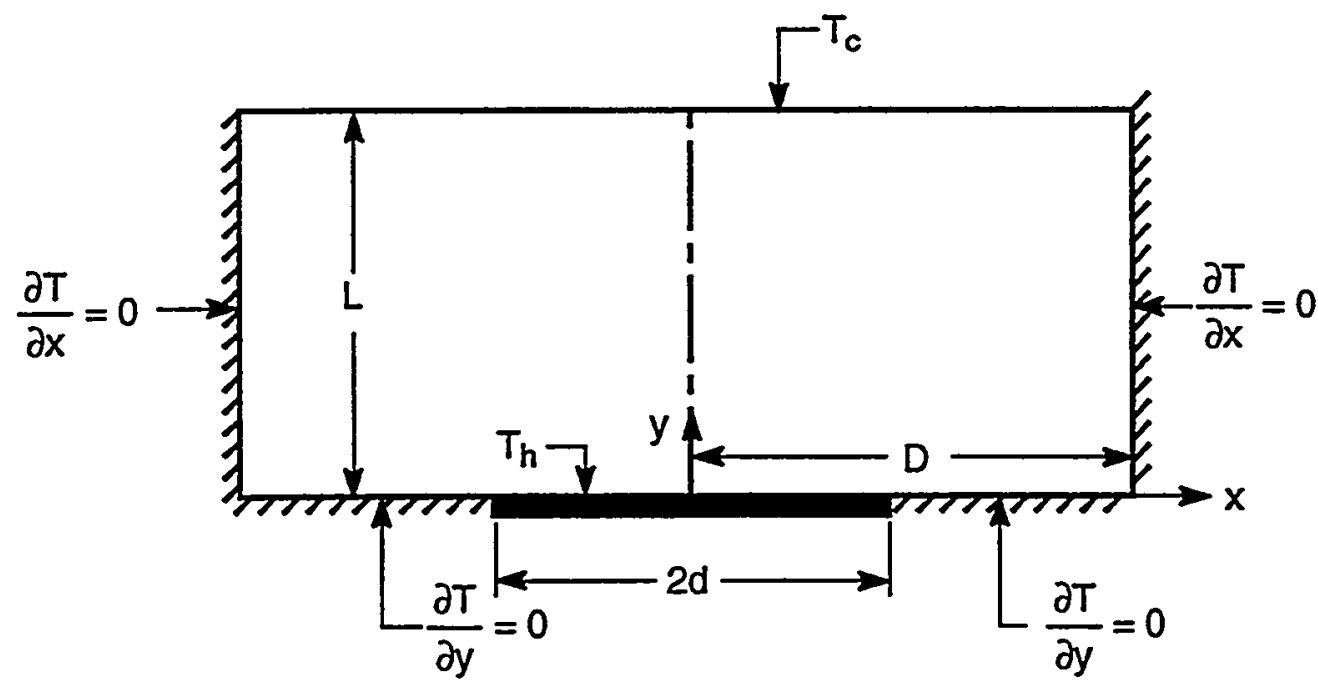

Figure 2-17.2D model of the overlying medium at a geologic repository (Kulacki and Keyhani, 1987) (Note: The geometric dimensions $d$ and $D$ are defined here and used in Figure 2-18).

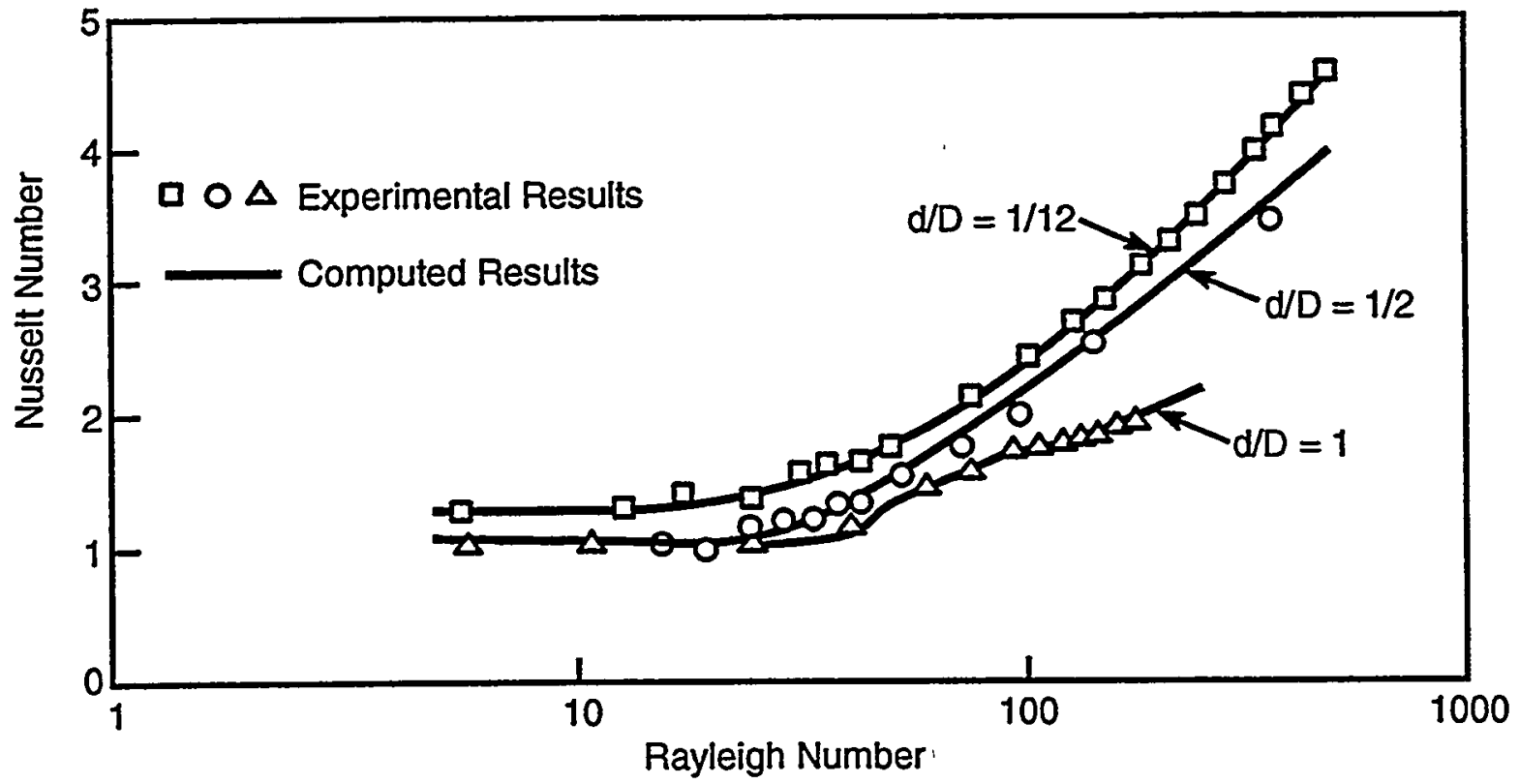

Figure 2-18. Experimental results which reflect repository conditions for a saturated, homogeneous, isotropic medium (see Figure 2-17) (Rajen and Kulacki, 1987). 
fluid conductive heat transfer. As such, the Nusselt number is a measure of the strength of the convective fluid flow. For a stagnant fluid, the Nusselt number is unity and the heat transfer is due to conduction. As the Rayleigh number increases, the convective flows increasingly influence the heat transfer, as seen by an increasing value for the Nusselt number.

The permeability of the medium used in Figure $2-18$ was $3.5 \times 10^{-9} \mathrm{~m}^{2}$, which is at least $2-5$ orders of magnitude larger than that at Yucca Mountain (see Table B-1). The porous medium Rayleigh number is proportional to the saturated permeability [see Eq. (2-5)]. Hence a typical repository scale Rayleigh number is expected to be 2-5 orders of magnitude smaller than those reported in Figure 2-18. From Figure 2-18, it is evident that at lower Rayleigh numbers, the Nusselt number is unity, indicating negligible hydrologic effects on thermal processes. Because of the partially-saturated condition and low medium permeability at Yucca Mountain, buoyancy-driven natural convection is anticipated to have a negligible effect on the overall heat transfer. This conclusion is supported by field experiments reported by Patrick et al. (1986), Zimmerman et al. (1986a), and Ramirez et al. (1991a) in which the heat transfer in the rock mass is frequently described as "conduction-dominated."

\subsubsection{Material Properties}

The moisture content of a porous medium frequently has an influence on the effective material properties, such as thermal conductivity and heat capacity. De Vries $(1963,1975)$, among others, has studied soils and reported semi-empirical correlations of the soil thermal conductivity and heat capacity as functions of mineral composition, porosity, and water content. Although theoretical models of effective properties are useful, direct experimental measurements are recommended.

Horton et al. (1983) and Horton and Wierenga (1984) have studied methods to measure the effective thermal conductivity of a porous medium, which include the cylindrical probe method and the microwave moisture-distribution method. These methods reflect continuing improvements in experimental techniques, especially in measuring partially-saturated thermal conductivity as a function of moisture content. To date, much attention has focused on methods for soils and modified techniques are being adapted for low-porosity tuffaceous rock.

Rasmussen et al. (1990) described a cylindrical probe method to measure thermal properties as functions of water content (see Figure 2-19). In this setup, a steady input is supplied to the heating unit and the temperature in the outer hole is measured and used to calculate the thermal conductivity. Rasmussen et al. (1990) reported that the thermal conductivity for Apache Leap tuff (similar to rock at Yucca Mountain) decreases by approximately 30 percent from fully-saturated to oven-dried conditions. The relationship between thermal conductivity and saturation was nonlinear, and became more important for drier samples. The influence of saturation on thermal conductivity is substantially less than for hydraulic conductivity (where it decreases by many orders of magnitude); however, the change can be important in determining the temperature field.

Radhakrishna et al. (1990) conducted thermal experiments simulating a backfilled disposal vault with a bentonite-sand buffer-backfill. The one-eighth scale model examined the thermal drying and rewetting of the medium. "Shrinkage cracks" in the buffer material occurred as a result of thermal drying. The rate of change in temperature increased as the drying zone increased, indicating a decrease in the thermal conductivity and thermal capacity of the buffer material. However, the drying zone and the 


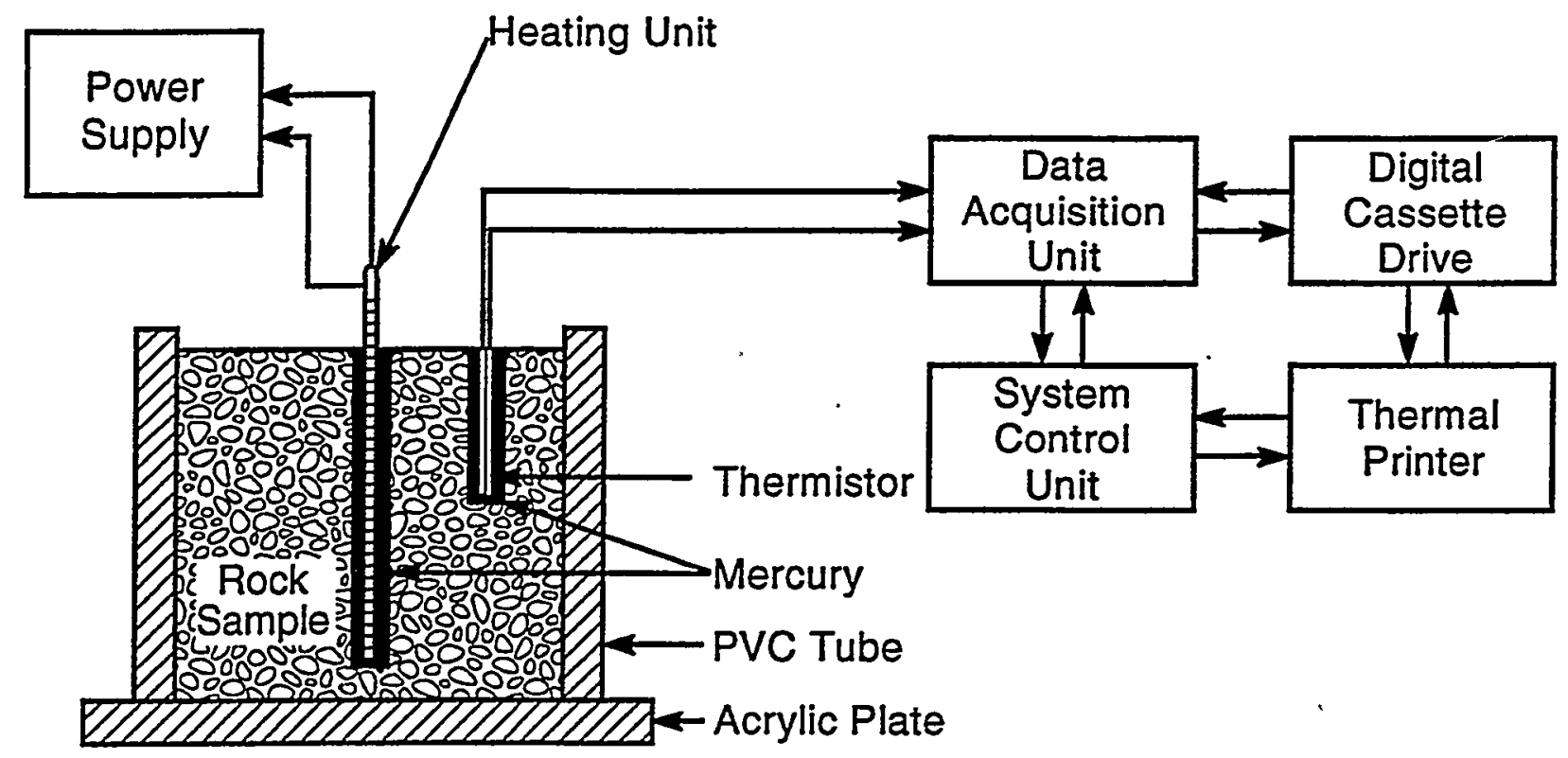

Figure 2-19. Experimental apparatus used to measure the thermal conductivity of Apache Leap tuff as a function of water content (Rasmussen et al., 1990).

formation of shrinkage cracks did not significantly decrease the efficiency of the buffer in its capacity to dissipate heat from the heater to the surrounding rock environment (Radhakrishna et al., 1990).

Although thermal properties have been noted to be influenced by changes in saturation (e.g., Rasmussen et al., 1990) and shrinkage cracks (e.g., Radhakrishna et al., 1990), it should be emphasized that these variations are probably small in comparison to the inherent and spatial uncertainties in thermal properties. For example, the rock mass thermal conductivities for the Topopah Spring welded tuff units are presented in Table 2-1 (DOE, 1990). The thermal conductivity is reported as a function of both (i) the thermal/mechanical unit (or simply the unit) and (ii) the degree of saturation (in situ or dry). A natural question is: What data are required in order to accurately predict the temperature field, given the present state-of-knowledge about (i) the variability in thermal conductivity within a single rock unit, (ii) the uncertainty in the spatial locations of the interfaces between rock units, (iii) the effect of changes in saturation on the rock thermal conductivity of a single rock unit, and (iv) the effect of "shrinkage cracks" on the rock thermal conductivity of a single rock unit?

It can be noted from Table 2-1 that the variation (i.e., mean plus-or-minus standard deviation) of the conductivity shows only a weak distinction between in situ and dry conditions, while a stronger distinction exists between the thermal/mechanical units. If the difference between mean values for the in situ and dry conditions is compared with the standard deviation (of either the in situ or dry conditions), then it can be concluded that the inherent uncertainty is at least comparable to the variation with saturation. In addition, there is typically a large uncertainty associated with the spatial locations of the interfaces between units. In summary, the inherent uncertainty and spatial variability of thermal properties are not negligible, and can be expected to overwhelm the variability in thermal properties due to changes in saturation and/or shrinkage cracks. 
Table 2-1. Rock mass thermal conductivity of selected units at Yucca Mountain (DOE, 1990)

\begin{tabular}{||c|c|c|c|c||}
\hline \multirow{2}{*}{$\begin{array}{c}\text { Thermal/ } \\
\begin{array}{c}\text { Mechanical } \\
\text { Unit }\end{array}\end{array}$} & \multicolumn{4}{|c|}{ Rock Mass Thermal Conductivity [W/(m-K)] } \\
\cline { 2 - 5 } & \multicolumn{2}{|c|}{ In Situ Saturation } & \multicolumn{2}{c||}{ Dry } \\
\cline { 2 - 5 } & $\begin{array}{c}\text { Mean } \\
\text { Value }\end{array}$ & $\begin{array}{c}\text { Standard } \\
\text { Deviation }\end{array}$ & $\begin{array}{c}\text { Mean } \\
\text { Value }\end{array}$ & $\begin{array}{c}\text { Standard } \\
\text { Deviation }\end{array}$ \\
\hline \hline TSw1 & & & & \\
\hline Lithophysae poor & 1.506 & 0.155 & 1.423 & 0.141 \\
\hline Lithophysae rich & 1.390 & 0.100 & 1.313 & 0.088 \\
\hline TSw2 & 1.910 & 0.083 & 1.839 & 0.064 \\
\hline TSw3 & 1.304 & 0.136 & 1.285 & 0.113 \\
\hline
\end{tabular}

\subsubsection{Computer Codes}

Computer codes that can be used to model $\mathrm{H} \Rightarrow \mathrm{T}$ coupled processes include TOUGH, VTOUGH, PORFLOW and others. Most of these codes model both $\mathrm{T} \Rightarrow \mathrm{H}$ and $\mathrm{H} \Rightarrow \mathrm{T}$ (i.e., $\mathrm{T} \Leftrightarrow \mathrm{H}$ ) coupled processes. These computer codes are discussed in Section 2.1.5, and the discussion is not repeated here.

\subsubsection{Summary and Conclusions}

In this section, the influence of hydrologic processes on thermal processes $(H \Rightarrow T)$ was reviewed. Specifically, four mechanisms of $\mathrm{H} \Rightarrow \mathrm{T}$ couplings were discussed in the areas of: (i) a heat pipe, (ii) condensate dripping, (iii) convective cooling, and (iv) thermal properties. Each coupling mechanism can be important, depending on specific conditions of the medium and processes.

The heat pipe is a coupling mechanism which efficiently transfers heat due to vaporization, pressure-driven vapor flow, condensation, and capillary-driven liquid flow. A distinguishing characteristic of a heat pipe is a plateau in the temperature profile (this is also a characteristic for gravity-driven condensate drainage into a boiling zone). The capillary-driven liquid flow in a heat pipe is expected to occur over a large area (Figure 2-11) as compared to localized areas for gravity-driven flow in fractures. For values of rock permeability relevant to Yucca Mountain, however, the heat pipe is not expected to be significant (Figure 2-13).

The gravity-driven condensate drainage in fractures into a boiling zone is another mechanism that can influence thermal processes. This coupling mechanism is very similar to the heat pipe, yet the difference is the dominant mechanism for liquid flow. In the heat pipe, it is capillary-driven flow in the matrix, and here it is gravity-driven flow in the fractures. This mechanism may provide a sufficient flow of liquid into a boiling zone so that the temperature is maintained at $100^{\circ} \mathrm{C}$ (i.e., a temperature plateau). The location and orientation of fractures is important in dictating where condensate flows. Hence, this mechanism is spatially localized, whereas the heat pipe mechanism is spatially distributed. This mechanism appears to be relevant to Yucca Mountain because the Topopah Spring unit is fractured and has low matrix permeability. 
Buoyancy-driven convective cooling is another mechanism for a $\mathrm{H} \Rightarrow \mathrm{T}$ coupling. However, the analyses in the literature suggest that natural convection at Yucca Mountain will have a negligible influence on thermal processes.

Thermal material properties (e.g., thermal conductivity) can also be influenced by hydrologic processes such as drying of the porous medium. There have been a number of studies which measure thermal properties of porous media such as tuffaceous rock. The effective thermal conductivity can be 30 percent lower for oven-dried tuff than for fully-saturated tuff, due to the lower conductivity of air compared with liquid water. Similarly, shrinkage cracks in drying buffer material have been experimentally reported. These changes, however, may be negligible because of the inherent uncertainty and spatial variability of thermal properties for the medium of interest at Yucca Mountain.

Overall, hydrologic processes may influence thermal processes through a number of coupling mechanisms. The couplings do appear plausible; however, most do not appear significant at Yucca Mountain. The most prominent coupling mechanism is gravity-driven condensate drainage in fractures, which depends strongly on the temperature field and fracture locations. It is conceivable that liquid condensate will drain near a number of waste containers, thereby maintaining the temperature near $100^{\circ} \mathrm{C}$ (plateau). The other coupling mechanisms are possible, yet less likely or less significant.

\section{$2.3 \quad$ THERMAL $\Rightarrow$ MECHANICAL}

The emplacement of spent nuclear fuel underground has the effect of generating expansion of the rock mass, increasing thermal stresses, and increasing the potential for normal and shear displacements of the fractures. The extent of thermally-induced stresses will depend on the thermal load, which in turn depends on the age of the waste at the time of emplacement, container spacing, etc. For high thermal loads, high temperature gradients are expected, hence high stress levels are expected near the waste emplacement containers. The thermal stresses are expected to persist during the life of the repository.

Three areas of discussion are included in this section: (i) the effect of temperature on mechanical properties of rock, (ii) thermally-induced stresses generated by the emplaced waste, and (iii) computer codes.

\subsubsection{Mechanical Properties and Material Strength}

Preliminary testing of the thermal properties of the host rock in the Topopah Spring geologic unit at Yucca Mountain has shown low coefficients of thermal expansion and higher-than-expected thermal conductivity properties (Nimick, 1990). Such properties, if shown to be true based on further site characterization studies, would be considered favorable conditions in accommodating the induced thermal stresses developed by emplacement of the waste. However, the effect of temperature on the mechanical properties of rock in the potential repository horizon is not well-determined at this time and needs further investigation. Mechanical properties of rock generally decrease with increasing temperature. Price et al. (1987) reported that, for samples from the potential repository horizon, Young's modulus shows an average decrease of 16 percent as the temperature is raised from 22 to $150^{\circ} \mathrm{C}$ at both 0 and $5 \mathrm{MPa}$ confining pressures.

Several preliminary analyses have also been conducted to determine the effect of thermal loading on the stability of underground excavations at Yucca Mountain. Arulmoli and St. John (1987), 
Christianson and Brady (1989), and Bauer and Costin (1990) have estimated temperature, stress, and deformation fields around emplacement holes in the potential repository. These studies conclude that spalling due to thermal stresses and fractures or slip along fractures is expected to be minor.

\subsubsection{Thermally-Induced Stresses}

Conductive heat transfer within a solid continuum is discussed briefly in Appendix A.1, with the 3D heat conduction equation given by Eq. (A-8). This equation is given in its simplest form by assuming constant thermal conductivity and no energy source/sink terms present. In partially-saturated rock with tightly packed joints and fractures, conduction through the medium, as governed by Eq. (A-8), is expected to be the primary mode of heat transfer. Several in situ heated block tests completed to date suggest that conductive heat transfer is in fact dominant and sufficient to describe temperatures in jointed rock (e.g., Zimmerman et al., 1986b and Voegele et al., 1981).

A change in the temperature of the medium results in a change in the state of stress and strain. In the case of linear elastic material behavior, the strain tensor can be assumed to consist of the sum of the components of strain due to an incremental change in temperature, and those strain components due to mechanical deformation within the rock alone. This relation can be expressed as

$$
e_{i j}=e_{i j}^{o}+e_{i j}^{\prime}
$$

where

$$
\begin{array}{ll}
\mathrm{e}_{\mathrm{ij}}^{\circ} & =\text { strain induced by an increment of temperature (dimensionless), and } \\
\mathrm{e}_{\mathrm{ij}}^{\prime} & =\text { strain induced by mechanical deformation [Eq. (C-5)] (dimensionless). }
\end{array}
$$

The temperature-induced strain $\left(\mathrm{e}_{\mathrm{ij}}^{\mathrm{o}}\right)$ can further be expressed as

$$
\mathrm{e}_{\mathrm{ij}}^{\mathrm{o}}=\alpha_{\mathrm{LTE}} \Delta \mathrm{T} \delta_{\mathrm{ij}}
$$

where

$$
\begin{array}{ll}
\alpha_{\mathrm{LTE}} & =\text { linear thermal expansion coefficient }\left(\mathrm{K}^{-1}\right) \\
\Delta \mathrm{T} & =\text { increment in temperature }(\mathrm{K}), \text { and } \\
\delta_{\mathrm{ij}} & =\text { Kronecker delta function. }
\end{array}
$$

The above relation represents a property of an isotropic body in which a change of temperature results in no change of shear strain, the only result being a change of volume of an elementary parallelepiped. Combining Eqs. (2-7) and (C-5) gives

$$
e_{i j}=\alpha_{L T E} \Delta T \delta_{i j}+\frac{1}{2 G}\left(\sigma_{i j}-\frac{v_{P}}{1+v_{P}} \sigma_{k k} \delta_{i j}\right)
$$

where

$$
v_{\mathrm{P}} \quad=\text { Poisson's ratio (dimensionless), }
$$




$$
\begin{array}{ll}
\sigma_{\mathrm{ij}} & =\text { components of stress tensor }\left(\mathrm{N} / \mathrm{m}^{2}\right), \\
\mathrm{G} & =\text { shear modulus }\left(\mathrm{N} / \mathrm{m}^{2}\right), \text { and } \\
\sigma_{\mathrm{kk}} & =\text { sum of normal stresses }\left(\sigma_{\mathrm{kk}}=\operatorname{Trace}(\sigma)=\sigma_{11}+\sigma_{22}+\sigma_{33}\right)\left(\mathrm{N} / \mathrm{m}^{2}\right) .
\end{array}
$$

which are known as the Duhamel-Neumann relations (Nowacki, 1962). Solving this system of equations with respect to the stresses results in

$$
\sigma_{i j}=2 G\left[e_{i j}+\frac{v_{P}}{.1-2 v_{P}}\left(e_{k k}-\frac{1+v_{P}}{v_{P}} \alpha_{L T E} \Delta T\right) \delta_{i j}\right]
$$

which gives six stress equations for $i$ and $j$ equal to 1,2 , and 3 . The second term within the parentheses is responsible for the thermally-induced portion of the stress.

Numerous studies have been conducted to determine the impact of thermal loading from an underground repository on the mechanical state. Shen and Stephansson (1990a) performed 3D numerical simulations with the computer code 3DEC, in which they modeled an area $2 \mathrm{~km} \times 2 \mathrm{~km} \times 2 \mathrm{~km}$. In this model, the repository heat loading was modeled with exponentially-decaying heat sources, and excavations were left out. They found that the rock stresses varied proportionally with the change in rock temperature, and reached a maximum after 200 yrs (when the rock temperature in the repository region was at its maximum). Compared to the in situ stress, the stress level increased in the repository region, while a decrease was seen further away. No tensile stresses developed within the model. Figure 2-20 shows the normal displacement response of fractures in both the horizontal and vertical orientations intersecting the repository region. The 3DEC analysis shows that the normal displacement due to thermal loading results in both horizontal and vertical fractures closing in the region around the repository. Outside this region, fractures were seen to open as much as $2 \mathrm{~mm}$.

Shen and Stephansson (1990b) conducted a thermomechanical analysis at the tunnel scale using the 3DEC code. The dimensions for this model were $25 \mathrm{~m} \times 25 \mathrm{~m} \times 18 \mathrm{~m}$, and the joint spacing was on the order of $1 \mathrm{~m}$. Their results showed that the response of the rock mass to the thermal effect was most significant in the vicinity of the tunnel and deposition hole. Large compressive stresses and tensile stresses were shown to develop in the tunnel roof when the temperature reached its maximum after 200 yrs. The tensile stresses were on the order of the tensile strength, indicating the potential for local rock failure. All joints were seen to close during the thermal loading period.

Costin and Chen (1991) performed a thermomechanical analysis of the G-tunnel heated block experiment using a 2D finite element model incorporating a compliant-joint rock mass model. Their main goal of the work was in the verification and validation of numerical models for simulating the behavior of a jointed rock mass subjected to thermal and mechanical loads. They were able to obtain good quantitative agreement between the temperatures calculated from the model and the temperatures measured in the heated block experiment, when they took into account the convective loss of heat from the top surface of the heated block. Comparison of the thermally-induced displacements between the finite element analysis and the heated block experiment provided much less quantitative agreement. The disagreement in the displacement results was considered to be due to uncertainties in the displacement measurements from some of the gauges, and the fact that a 2D analysis was conducted of a truly $3 \mathrm{D}$ experiment. In addition, the compliant-joint model assumes two sets of perpendicular joints and may not have been truly representative of the jointing in the actual block itself. 


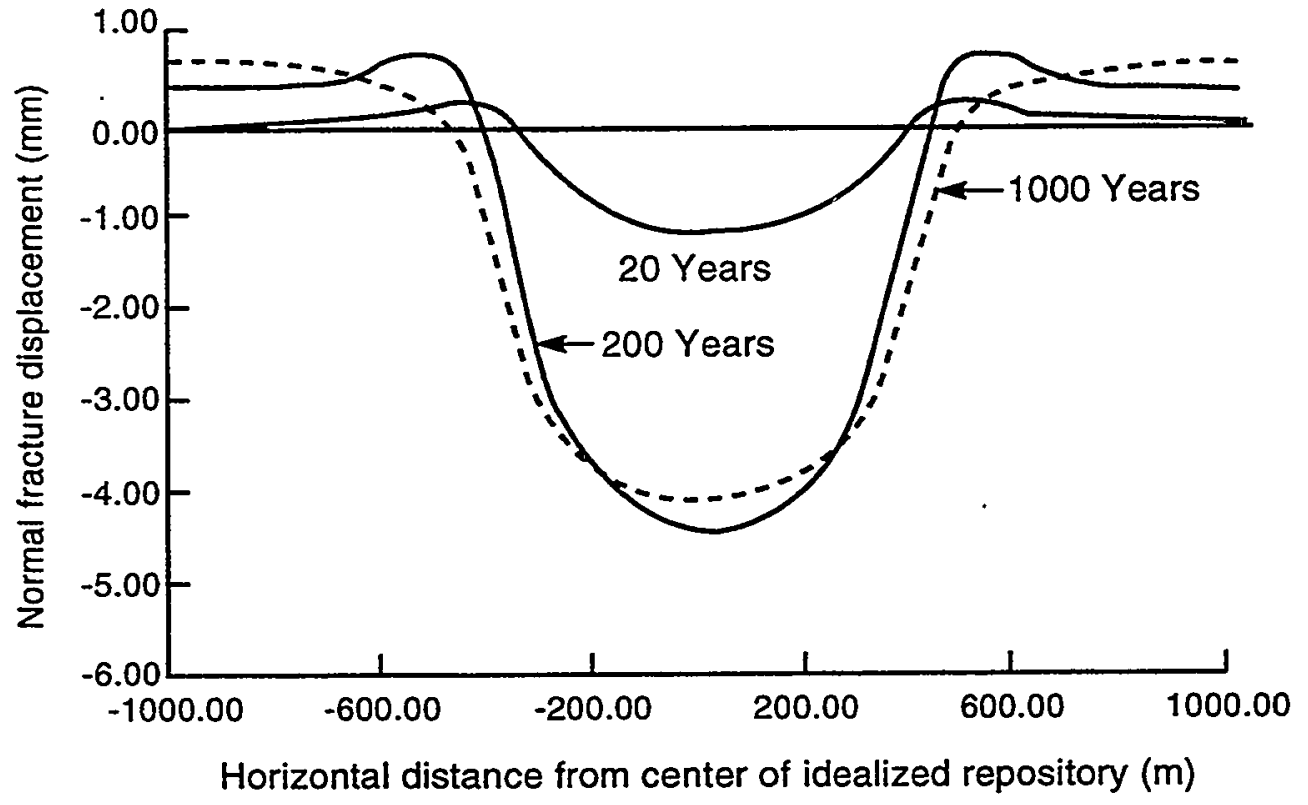

(a)

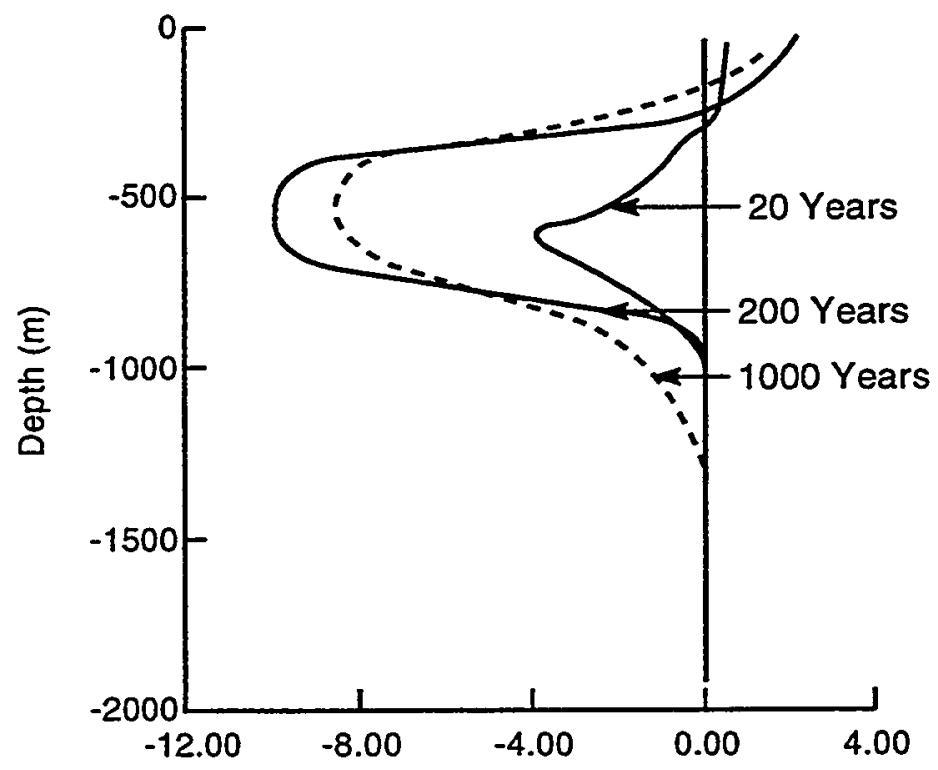

Normal fracture displacement $(\mathrm{mm})$

(b)

Figure 2-20. Thermally-induced normal fracture displacement (a) for horizontal fracture zone just beneath the repository and (b) of vertical fracture zone intersecting repository. A positive sign indicates opening displacement (Shen and Stephansson, 1990a). 
Bauer et al. (1991) performed 2D and 3D numerical analyses using the code STRES3D to predict thermal and stress environments in the vicinity of repository openings at Yucca Mountain. For the proposed power density and emplacement schedule for the waste, their results showed, in general, that openings close to heat sources would attain high temperatures and experience a significant stress increase. They considered stresses resulting from in situ, thermal, and seismic effects in designing the drifts, and found that, for all drift types, the thermal stress is the greatest horizontal component included in the combination. Hardy and Bauer (1991) reported that the horizontal stress at the repository horizon can change from the initial conditions of 3 to $6 \mathrm{MPa}$ up to 15 to $20 \mathrm{MPa}$, depending on the design assumptions and host rock properties. As a consequence, this thermal loading would require unique drift design considerations to reduce deterioration of the rock throughout the repository operational period. Figure 2-21 illustrates the stress history at the waste emplacement drift and tuff main drift for the Yucca Mountain repository, and shows that the thermal loading of a drift location around the repository is very location-dependent (Hardy and Bauer, 1992). The tuff main drift sees reduction in vertical stress but a significant increase in horizontal stress, even though its temperature rise is modest. This is due to thermal expansion of the rock mass in waste emplacement regions which surround the mains.

\subsubsection{Computer Codes}

In most of the computer codes that can perform a coupled thermomechanical analysis, the coupling is assumed to be one-way (i.e., thermal affecting mechanical). This is primarily done because the thermal to mechanical coupling is dominant, causing changes not only in stresses and strains, but also in mechanical properties and material strength. The effect of mechanical changes on thermal properties is generally assumed to be negligible. Many computer codes perform a thermomechanical analysis in a sequentially-coupled manner. For instance, a heat transfer code is used to obtain the temperature distribution, which is then input into another mechanical code to calculate the temperature-induced stresses. This approach is justifiable when performing linear elastic stress analysis; however, most rock joints behave nonlinearly. Thus, when explicitly modeling jointed rock mass behavior, one needs to apply the thermal loading in an incremental and realistic manner, while updating the mechanical state after each thermal loading increment. The following is a short list of some of the available computer codes applicable to the HLW program that can perform a coupled thermomechanical analysis. The list includes only those codes that are considered most applicable to the HLW program, and can perform both thermal and thermomechanical analyses.

\subsubsection{3DEC}

The 3D discrete element code 3DEC (Cundall, 1988) can model the effects of thermal and mechanical loadings on a jointed rock mass. It employs an explicit time-marching scheme to model the rock mass behavior. The blocks can be either rigid or deformable with deformable contacts. The heat transfer is based on conduction within the medium with the provision for temperature, flux, convective, or radiative boundaries. Only one-way coupling from thermal to mechanical is considered. The code also has provisions for adopting an implicit finite difference scheme. The approach used in performing a coupled analysis is to first run the problem out to some specified number of thermal time steps. At this point, the thermal or real time is held constant while mechanical cycling is performed to reach a new equilibrium state. This process is then continued. The requirement in performing the simulation in this manner is that temperature increases between successive thermal time steps cause only "small" out-ofbalance forces in the blocks, especially for nonlinear problems. 


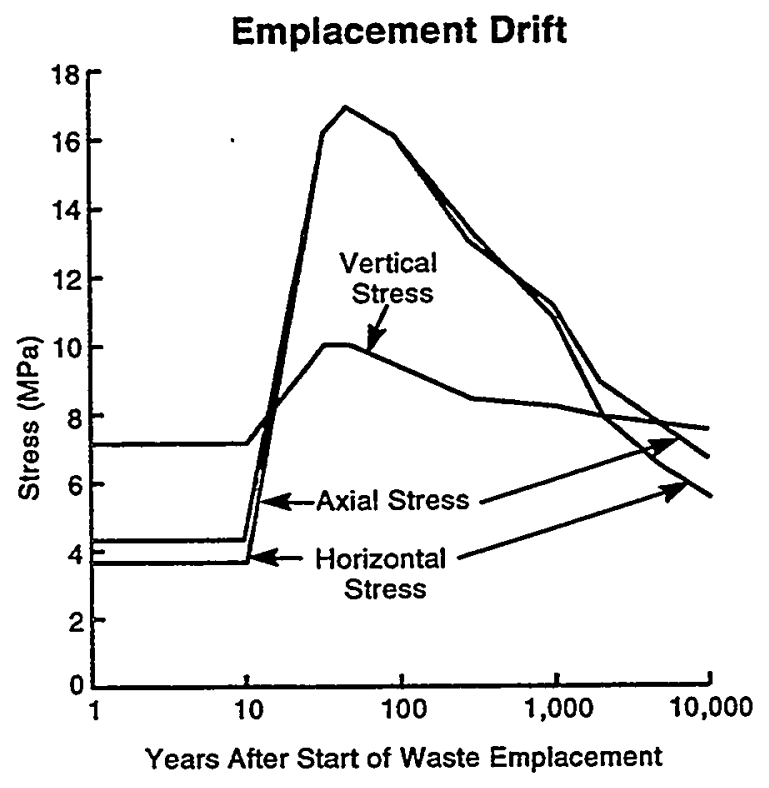

(a)

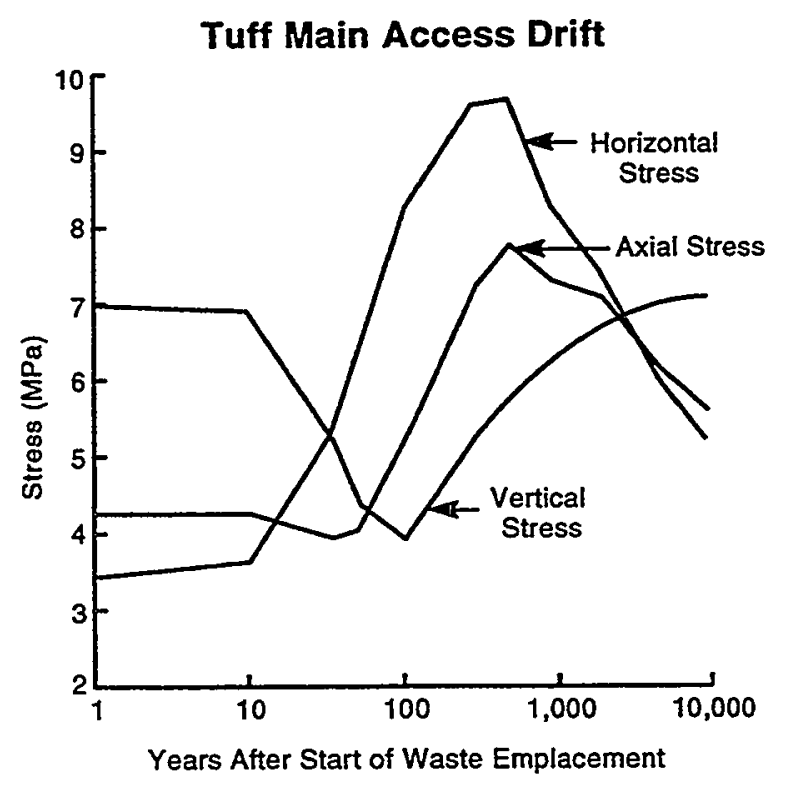

(b)

Figure 2-21. Thermal-mechanical environment in the vicinity of (a) waste emplacement drift and (b) tuff main access drift at the proposed repository beneath Yucca Mountain (Hardy and Bauer, 1992). Reprinted from: W.R. Wawersik (ed.), 33rd US Rock Mechanics Symposium--Proceedings, Santa Fe, New Mexico, 8-10 June 1992. 1992. 1157 pp. Hfl.150/US \$85.00. Please order from: A.A. Balkema, Old Post Road, Brookfield, Vermont 05036 (telephone: 802-276-3162; telefax: 802-2763837). 


\subsubsection{SPECTROM-32}

SPECTROM-32 is a finite element program for analyzing $2 \mathrm{D}$ and axisymmetric inelastic thermomechanical problems related to the geologic disposal of nuclear waste (Callahan et al., 1989). The code is part of the SPECTROM (Special Purpose Engineering Codes for Thermal/ROck Mechanics) series of special-purpose computer programs developed by RE/SPEC, Inc., to address many unique rock mechanics problems encountered in analyzing radioactive wastes stored in geologic formations. The principal component models used in the SPECTROM-32 program involve thermoelastic, thermoviscoelastic, thermoelastic-plastic, and thermoviscoplastic types of material behavior. Special material considerations provide for the incorporation of limited tension material behavior and consideration of jointed material behavior. Numerous program options provide the capabilities for various boundary conditions, sliding interfaces, excavation, and backfill, which are important to the geologic disposal of radioactive wastes.

\subsubsection{STRES3D}

STRES3D is a semi-analytical, thermomechanical analysis code for predicting transient temperatures, stresses, and displacements in infinite or semi-infinite, conducting, homogeneous, elastic media. The code is based on thermoelastic analytic solutions for point heat sources in an infinite body. A semi-infinite body is treated by superposition of source and sink terms coupled with relief of surface shear stresses by numerical integration. For semi-infinite problems, the program models the earth surface as isothermal and stress free. In a recent development, numerical interaction of the point heat source solution has been applied so that line or plate heat sources can be represented at improved accuracy and reduced computational effort. This development has proved useful when modeling large-scale nuclear waste repository layouts. An important feature of the code is that time is dealt with analytically, which means that a solution for any instant in time may be obtained for equal computational effort and cost. The code has been shown to be efficient in modeling the temperatures and thermally-induced stresses generated from a typical repository layout containing a large number of waste emplacement panels (e.g., see Bauer et al., 1991).

\subsubsection{FLAC}

FLAC (Fast Lagrangian Analysis of Continua) is a 2D finite difference program for modeling soil and rock behavior (Board, 1989a). FLAC has features including: (i) models for large-strain plasticity, strain-softening, and volumetric yielding models, (ii) cable elements for modeling reinforcement, (iii) interfaces to represent joints, (iv) automated mesh generation, (v) groundwater flow and consolidation, and (vi) optional creep and thermomechanical modeling capabilities. The coupling capabilities within FLAC include $\mathrm{T} \Rightarrow \mathrm{M}, \mathrm{H} \Rightarrow \mathrm{M}$, and $\mathrm{M} \Rightarrow \mathrm{H}$. THM coupled modeling can be accomplished within FLAC by first determining the thermomechanical response, followed by cycling to hydrologic-mechanical equilibrium. In the case of nonlinear behavior, small thermal steps must be taken in order to obtain accurate results.

\subsubsection{MSC/NASTRAN}

MSC/NASTRAN (MacNeal, 1972) is a general purpose finite element computer program for structural analysis. The types of analysis in the program include: static response to concentrated and distributed loads, to thermal expansion and to enforced deformation; dynamic response to transient loads, to steady-state sinusoidal loads and to random excitation; determination of real and complex eigenvalues 
for use in vibration analysis, dynamic stability analysis, and elastic stability analysis. The program includes limited capability for the solution of nonlinear problems, including piecewise linear analysis and nonlinear static response and transient analysis of nonlinear dynamic response. In addition to the wide range of structural element types within the code, MSCINASTRAN also can model a discontinuity utilizing joint elements. MSC/NASTRAN also has heat flow capability which may be used either as a separate analysis to determine temperatures and fluxes or to determine temperature inputs for structural problems. Steady and transient problems can be solved, including heat conduction (with variable conductivity for static analysis), film heat transfer, and nonlinear (fourth power law) radiation.

\subsubsection{STEALTH}

STEALTH (Hofmann, 1981a) is a system of computer programs, which is designed, based on an explicit finite element method, to handle fluid-structure interaction, soil-structure interaction, and/or simultaneous thermal and mechanical events. It is capable of solving coupled thermal transient and quasistatic mechanical problems. STEALTH is developed for large strain and non-ideal constitutive behavior. It has 1D, 2D, and 3D versions. Automatic rezoning capability is provided in the codes. User defined material models are allowed in the analysis; for example, functional forms to describe the pressure dependence of the shear modulus or the temperature dependence of conductivity are available in the code. Discontinuities for a $2 \mathrm{D}$ problem are simulated by the codes using slidelines whose behavior is governed by the assigned material properties, cohesion, and friction.

\subsubsection{GPBEST3D}

GPBEST3D (GPBEST, 1992) is a general purpose boundary element program capable of analyzing axisymmetric, 2D and 3D mechanical and thermomechanical problems. The program can solve quasi-static, periodic dynamic, and transient time domain dynamic problems. The elastic analyses include isotropic or cross anisotropic media. The inelastic analyses include isotropic plasticity with variable hardening, kinematic plasticity with multiple yield surfaces, elastoplasticity under cyclic loading, thermoplasticity, thermoviscoplasticity, and anisotropic elastoplasticity. Frictional interfaces can be used to model discontinuities in the medium. Thermal boundary conditions include assignment of temperatures to different nodes at different instants, time dependent temperature variation, and hot spots or local heat sources.

\subsubsection{BEASY}

BEASY (BEASY, 1993) is a boundary element program for analysis of axisymmetric, 2D and 3D mechanical and thermomechanical problems. The program can carry out multi-zone analysis to handle different material types and various geometric features of the problem. BEASY automatically uses discontinuous elements where it needs the extra accuracy. Isotropic, orthotropic, and anisotropic material properties can be handled by the program. Discontinuities in the problem domain is modeled as slide lines. Line and point heat sources can be included in the model.

\subsubsection{ADINA}

ADINA (ADINA R\&D, 1992) is a finite element package for modeling 2D and 3D problems in structural analysis, heat transfer and fluid flow. The package is composed of separate modules, where ADINA performs stress and displacement analysis, ADINA-T performs analysis of heat transfer and field problems, and ADINA-F performs analysis of incompressible viscous fluid flow. Coupling back to ADINA 
is provided for both ADINA-T and ADINA-F. The main program ADINA can provide analysis for linear and nonlinear problems in statics and dynamics, including contacts. ADINA-T provides steady-state and transient heat transfer analysis with time or temperature dependent properties and convection or radiation boundary conditions. ADINA-F provides steady-state and transient viscous fluid flow (with or without heat transfer), natural and forced convection, as well as flow through porous media.

\subsubsection{ANSYS}

ANSYS (Swanson Analysis Systems, Inc., 1991) is a 2D and 3D finite element program having structural, thermal, as well as fluid flow capabilities. The structural capabilities include both static and dynamic analyses using a wide variety of elements. The thermal capabilities include steady-state, transient, phase change, as well as thermal-structural interaction. Fluid flow in the form of potential flow and seepage can be simulated, in addition to fluid-structural interactions. ANSYS is capable of modeling 2D and 3D contact surfaces with friction. The ANSYS element library contains more than 90 distinct elements as well as numerous material modules.

\subsubsection{Summary and Conclusions}

In this section, the influence of the temperature field on mechanical processes has been discussed. In particular, in the near field the temperature rise in the rock adjacent to the borehole has been predicted to approach $200^{\circ} \mathrm{C}$ during the first 100 yrs (O'Neal et al., 1984). The thermally-induced stresses will most likely be larger than those due to the in situ stress field or induced by mechanical excavation and seismic events (Bauer et al., 1991). As a consequence, it appears that thermal loads need to be considered in drift design and stability analyses.

The computer codes listed have been widely used and give the analyst many options in modeling the long-term mechanical behavior of a repository under thermal loading, assuming either continuum or discontinuum rock mass behavior. Thermomechanical studies using a few of these codes (e.g., 3DEC, STRES3D) have been discussed in this section. These codes have many limitations, and many options of these codes may need verification and validation against well-controlled laboratory or field experiments. Experimental and field studies on the impact of temperatures on mechanical properties and long-term mechanical degradation of the rock types at Yucca Mountain will be needed. Much of the evaluation of long-term thermal degradation of excavations within rock types similar to that at Yucca Mountain are based on computer studies, and also need experimental and field validation.

\subsection{MECHANICAL $\Rightarrow$ THERMAL}

Mechanical processes are characterized through the stress, strain and/or displacement fields, and thermal processes are characterized through the temperature field. The objective of this section is to review how mechanical processes may influence thermal processes $(M \Rightarrow T)$ at Yucca Mountain. In the literature, the $\mathrm{M} \Rightarrow \mathrm{T}$ coupling has not been considered important, and is rarely mentioned in consideration of Yucca Mountain. The mechanical processes are thought to influence thermal processes by primarily changing fracture apertures, hence changing the effective thermal conductivity. Frictional dissipation of mechanical energy is another way which is analogous to viscous dissipation discussed in the subject of fluid mechanics (Bird et al., 1960). However, no literature considered frictional dissipation to be important, hence it is not considered here. 
Lin et al. (1991) note that fractures were experimentally observed to have some effect on the temperature field. Thermocouple readings indicated lower temperatures for locations behind fractures (i.e., fractures located between the thermocouple and the heat source). The fractures increased the effective thermal resistance to conductive heat transfer. Lin et al. (1991) conclude that the measured temperatures agree with the predictions, with minor variations in temperature due primarily to the presence or absence of fractures. Although the influence of fractures was observed, they were assessed to be insignificant with respect to the overall temperature field (Lin et al., 1991).

Mechanical processes may influence hydrologic and/or chemical processes, which then influence thermal processes, yet this is considered a sequence of couplings and not a direct coupling (hence it is not considered in this section, but in other sections). For example, mechanical processes are expected to change fracture apertures, which may affect condensate drainage, which then may affect the temperature field. As such, this would be an $M \Rightarrow H \Rightarrow T$ sequence of couplings, which would be discussed in this report as two one-way couplings, that is, $M \Rightarrow H$, and $H \Rightarrow T$. There is little to no discussion in the literature of direct $M \Rightarrow T$ couplings, primarily because there is no evidence that it is important.

\subsection{THERMAL $\Rightarrow$ CHEMICAL}

The geochemical system is an important part of the natural barriers at Yucca Mountain. As suggested by the name thermodynamics, geochemical processes are strongly influenced by the temperature of the environment in which the process is operating. As discussed in Appendix D, most of the thermodynamic properties used in calculating geochemical equilibrium (equilibrium constants, free energies, activity coefficients) are nonlinear functions of temperature. Rates of reaction are also dependent on temperature through the Arrhenius rate equation. Most of these relationships are well-defined by thermodynamic and kinetic theory, and are discussed only briefly below. The reader is referred to a variety of textbooks and review articles (e.g., Garrels and Christ, 1965; Moore, 1972; Alberty and Daniels, 1979; Lasaga and Kirkpatrick, 1981; Stumm and Morgan, 1981; Lasaga, 1984) for a more extensive and complete treatment of the effects of temperature.

\subsubsection{Thermal Effects on Equilibrium Chemistry}

\subsubsection{Equilibrium Constants $(\log , \mathrm{K})$}

For any chemical reaction at equilibrium, the thermodynamic relationships, as described in Appendix D, are applicable. As a function of temperature, the standard Gibbs free-energy change $\Delta G_{R}^{\circ}(\mathrm{J} / \mathrm{mole})$ is defined by the relationship

$$
\Delta \mathrm{G}_{\mathrm{R}}^{\circ}=\Delta \mathrm{H}_{\mathrm{R}}^{\circ}-\mathrm{T} \Delta \mathrm{S}_{\mathrm{R}}^{\circ}
$$

where

$$
\begin{array}{ll}
\Delta \mathrm{H}_{\mathrm{R}}^{\circ} & =\text { standard enthalpy or heat content change of reaction (J/mole), } \\
\Delta \mathrm{S}_{\mathrm{R}}^{\circ} & =\text { standard entropy change of reaction }[\mathrm{J} /(\text { mole- } \mathrm{K})], \text { and } \\
\mathrm{T} & =\text { temperature }(\mathrm{K}) .
\end{array}
$$


At a given temperature, $\Delta \mathrm{H}_{\mathrm{R}}{ }^{\circ}$ and $\Delta \mathrm{S}_{\mathrm{R}}{ }^{\circ}$ are determined by the relationships

$$
\begin{aligned}
\Delta \mathrm{H}_{\mathrm{R}}^{\circ} & =\sum \Delta \mathrm{H}_{\mathrm{F}, \text { products }}^{\mathrm{o}}-\sum \Delta \mathrm{H}_{\mathrm{F}, \text { reactants }}^{\mathrm{o}} \\
\Delta \mathrm{S}_{\mathrm{R}}^{\circ} & =\sum \mathrm{S}_{\mathrm{F}, \text { products }}^{\mathrm{o}}-\sum \mathrm{S}_{\mathrm{F}, \text { reactants }}^{\circ}
\end{aligned}
$$

where

$$
\begin{array}{ll}
\Delta \mathrm{H}_{\mathrm{F}}^{\circ} \quad= & \text { enthalpy of formation of a compound from its elements in their standard state } \\
& (\mathrm{J} / \mathrm{mole}), \text { and } \\
\mathrm{S}_{\mathrm{F}}^{0} & =\text { entropy of formation of a compound from its elements in their standard state } \\
& {[\mathrm{J} /(\text { mole- } \mathrm{k})] .}
\end{array}
$$

Both $\Delta \mathrm{H}_{\mathrm{R}}^{\circ}$ and $\Delta \mathrm{S}_{\mathrm{R}}^{\circ}$ are also functions of temperature. The values of $\Delta \mathrm{H}_{\mathrm{F}}^{\circ}$ and $\Delta \mathrm{S}_{\mathrm{F}}^{\circ}$ necessary to calculate changes in enthalpy and entropy of reaction are available for a number of common minerals and species, at a variety of temperatures, and can be simply looked up or interpolated for the temperature of interest. Alternatively, at constant pressure, the heat capacity $c_{\mathrm{P}}[\mathrm{J} /(\mathrm{mole}-\mathrm{K})]$ can be used to extrapolate one temperature $\left(T_{1}\right)$ to higher temperature $\left(T_{2}\right)$ through the relationships

$$
\Delta H_{R, T_{2}}-\Delta H_{R, T_{1}}=\int_{T_{1}}^{T_{2}} \Delta c_{P} d T
$$

and

$$
S_{T_{2}}-S_{T_{1}}=\int_{T_{1}}^{T_{2}} c_{P} d \ln T
$$

where

$$
\Delta c_{\mathrm{P}}=\sum \mathrm{c}_{\mathrm{P}, \text { products }}-\sum \mathrm{c}_{\mathrm{P}, \text { reactants }}
$$

Fit to experimental data, $c_{\mathrm{p}}$ is commonly reported for solids as an empirical power function of absolute temperature with the general form $\mathrm{a}+\mathrm{bT}+\mathrm{cT}^{-2}(\mathrm{a}, \mathrm{b}, \mathrm{c}=$ empirical constants), and is readily integrated (Garrels and Christ, 1965).

When $\Delta \mathrm{H}_{\mathrm{R}}^{\circ}$ and $\Delta \mathrm{S}_{\mathrm{R}}^{\circ}$ are determined at the desired temperature, they can be substituted in Eq. $(2-10)$ and the standard free energy of reaction can be calculated at the new temperature. Once $\Delta G_{R}^{\circ}$ is obtained, the equilibrium constant $(K)$ for the reaction can then be calculated using the relationship

$$
\Delta G_{R}^{o}=-R_{I} T \ln (K)
$$


In practice, the rigorous thermodynamic approach outlined above is frequently simplified. The van't Hoff equation has been developed to calculate $\log \mathrm{K}$ values at a new temperature (in Kelvin) according to the relationship

$$
\log \mathrm{K}_{\mathrm{T}_{2}}=\log \mathrm{K}_{\mathrm{T}_{1}}-\frac{\Delta \mathrm{H}_{\mathrm{R}}^{\circ}}{2.303 \mathrm{R}_{\mathrm{I}}}\left(\frac{1}{\mathrm{~T}_{2}}-\frac{1}{\mathrm{~T}_{1}}\right)
$$

This relationship assumes that the standard enthalpy of reaction remains constant over the temperature range of interest. According to this relationship, $\log K$ plotted against $1 / T$ will yield a straight line with

a slope of $-\Delta H_{R}^{\circ} / 2.303 R_{I}$. In practice, $T_{1}=25^{\circ} \mathrm{C}(=298 \mathrm{~K})$ is commonly used as a reference temperature. Since $\Delta \mathrm{H}_{\mathrm{R}}^{\circ}$ can only be safely assumed constant over a narrow temperature interval for many reactions, use of Eq. (2-16) is valid only over small temperature intervals. Allison et al. (1990) point out that the van't Hoff equation can lead to significant errors in extrapolating from a $25^{\circ} \mathrm{C}$ reference to temperatures on the order of $100^{\circ} \mathrm{C}$ or greater.

An alternative approach is used in several thermogeochemical models (Wolery, 1979; Morrey, 1988; Allison et al., 1990). This involves fitting the $\log \mathrm{K}$ of a given reaction to a power function of temperature. This may be accomplished by tabulating data determined over a range in experimental temperatures and curve-fitting, or else by performing the analysis as outlined above and recasting the log $\mathrm{K}$ results in terms of an appropriate mathematical expression. As an example, Allison et al. (1990) proposed an expression of the form

$$
\log \mathrm{K}_{\mathrm{T}}=\mathrm{A}+\mathrm{BT}+\mathrm{C} / \mathrm{T}+\mathrm{D} \log (\mathrm{T})+\mathrm{ET} \mathrm{T}^{2}+\mathrm{F} / \mathrm{T}^{2}+\mathrm{GT} \mathrm{T}^{\frac{1}{2}}
$$

where

A, B, C, D, E, F, and G = empirical coefficients determined for the reaction of interest, and $\mathrm{T}=$ temperature $(\mathrm{K})$.

\subsubsection{Activity Coefficients}

In electrolyte solutions, activity coefficient calculations are commonly expressed by an equation such as the extended Debye-Hückel approximation (Stumm and Morgan, 1981)

$$
\log \left(\gamma_{i}\right)=\frac{-\mathrm{Az}_{\mathrm{i}}^{2} \sqrt{\mathrm{I}}}{1+\mathrm{Ba}_{\mathrm{i}} \sqrt{\mathrm{I}}}+\mathrm{b}_{\mathrm{i}} \mathrm{I}
$$

where

$$
\gamma_{\mathrm{i}}=\text { activity coefficient of species (ion) } \mathrm{i}\left(\mathrm{kg} \mathrm{H}_{2} \mathrm{O} / \mathrm{mole} \mathrm{i}\right)
$$




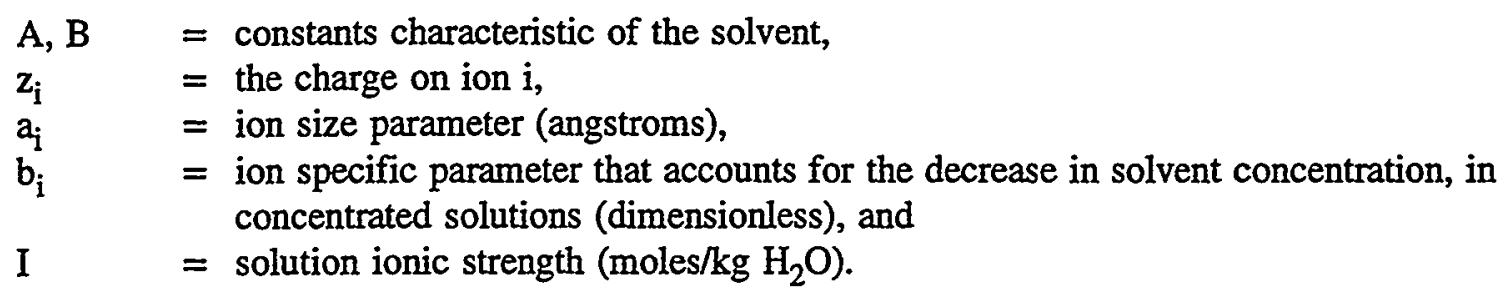

For water as a solvent, $\mathrm{A}$ and $\mathrm{B}$ depend on the dielectric constant $\varepsilon$ (dimensionless) and the absolute temperature T (Kelvin) of the solution (Stumm and Morgan, 1981)

$$
A=1.82 \times 10^{6}(\varepsilon T)^{-\frac{3}{2}}
$$

and

$$
\mathrm{B}=50.3(\varepsilon \mathrm{T})^{-\frac{1}{2}}
$$

For dilute solutions, the effect of temperature on the activity coefficient is minor. At higher electrolyte concentrations, the effect of temperature is more pronounced, but the direction of the shift in the activity coefficient with temperature will depend on the species under consideration (Stumm and Morgan, 1981).

For many reactions, $\Delta c_{P}$ is close to zero and $\Delta H_{R}^{0}$ is essentially independent of temperature [Eq. (2-14)]. In these instances, the van't Hoff relationship [Eq. (2-17)] is applicable and log $\mathrm{K}$ vs. 1/T will result in a linear plot. Stumm and Morgan (1981) stress that the applicability of these assumptions, and the magnitude and direction of the shift in thermodynamic equilibrium with temperature, is dependent on the thermodynamic properties of the species and phases involved in the reaction being considered. The solution composition, partial gas pressures, solid-solution, and the total pressure of the system will all help to condition the overall effect of temperature.

\subsubsection{Temperature Effects on Nonequilibrium Chemistry}

Lasaga (1981a) notes that reaction rate constants can increase by several orders of magnitude with temperature increases on the order of $100^{\circ} \mathrm{C}$. This is due to the higher potential energy of molecules and ions at higher temperatures. This additional energy increases the likelihood that molecules and ions involved in reaction will cross over potential barriers to reaction, leading to higher reaction rates. For example, experimental evidence suggests that the rate constant $\left(k_{r}\right.$ in $\left.s^{-1}\right)$ for many reactions varies exponentially with increasing temperature. As described in Appendix $\mathrm{D}$, this dependence is commonly expressed in terms of the Arrhenius equation, such that 


$$
k_{r}=A_{P} \exp \left(-\frac{E_{a}}{R_{I} T}\right)
$$

where

$$
\begin{array}{ll}
A_{\mathrm{P}} & =\text { pre-exponential factor }\left(\mathrm{s}^{-1}\right), \\
\mathrm{E}_{\mathrm{a}} & =\text { activation energy }(\mathrm{J} / \text { mole }), \\
\mathrm{R}_{\mathrm{I}} & =\text { ideal gas constant }[8.3147 \mathrm{~J} /(\text { mole- } \mathrm{K})], \text { and } \\
\mathrm{T} & =\text { absolute temperature }(\mathrm{K}) .
\end{array}
$$

Lasaga (1981a) notes that the pre-exponential factor $A_{P}$ may vary weakly as a function of temperature (Appendix D). In general, however, it is difficult to separate the effects of a temperature-dependent preexponential factor from the uncertainties inherent in the experimental data. If the activation energy $\mathrm{E}_{\mathrm{a}}$ is assumed to be constant with respect to temperature, it can be seen from Eq. (2-22) that a plot of In $\mathrm{k}$ vs. $1 / T$ results in a straight line with a slope of $E_{a} / R_{I}$ and an intercept of $A_{p}$. However, it is possible that $E_{a}$ varies with temperature, in which case the $\ln \left(\mathrm{k}_{\mathrm{T}}\right)-1 / \mathrm{T}$ plot would be curved and the simple expression in Eq. (2-21) is not strictly applicable. In addition, changes in reaction mechanisms may lead to a discontinuous variation of $\mathrm{E}_{\mathrm{a}}$ with temperature.

Expressing the rate of mineral dissolution over an effective surface area in terms of moles of reactant mineral destroyed per second, Helgeson et al. (1984) used transition state theory to propose that at a constant pressure, the rate constant $\left(\mathrm{k}_{\mathrm{r}}\right)$ varies with temperature such that

$$
k_{r}\left(T_{2}\right)=\frac{T_{2} k_{r}{ }^{0}}{T_{1}} \exp \left[\frac{-\Delta H_{A C T}}{R_{I}}\left(\frac{1}{T_{2}}-\frac{1}{T_{1}}\right)\right]
$$

where

$$
\begin{aligned}
& \mathrm{T}_{1} \quad=\text { original temperature }(\mathrm{K}), \\
& \mathrm{k}_{\mathrm{r}}^{0} \quad=\text { rate constant at } \mathrm{T}_{1}\left(\text { moles }-\mathrm{cm}^{-2}-\mathrm{s}^{-1}\right), \text { and } \\
& \Delta \mathrm{H}_{\mathrm{ACT}}=\text { enthalpy of activation }(\mathrm{J} / \mathrm{mole}) .
\end{aligned}
$$

\subsubsection{Computer Codes}

A number of computer codes have been developed to calculate geochemical equilibria and, to some extent, reaction kinetics. All of the codes require an appropriately formatted database containing the thermodynamic properties of the minerals, gases, and aqueous species to be considered. Particular emphasis will be placed on the abilities of the various codes to handle temperature variations. While the codes are generally not coupled to thermal processes, to the extent that they are able to simulate the convective, conductive, and radiative heat transfer associated with a heat source, they are able to consider the effects of temperature on reaction thermodynamics. The codes have been summarized, compared, and contrasted extensively for a variety of problem types (e.g., Nordstrom et al., 1979; Nordstrom and Ball, 1984; Kincaid et al., 1984a,b; Nordstrom and Munoz, 1985; Serne et al., 1990; Mangold and Tsang, 1991), 
and only a brief summary of the operational features of more commonly used and readily available codes will be given below.

Geochemical codes have generally followed one of two approaches to solve for chemical speciation (i) minimization of $\Delta \mathrm{G}_{\mathrm{R}}^{0}$, or (ii) mass action/mass balance using equilibrium constants. The codes listed here all employ the second technique. Morrey, et al. (1986) note that, because the thermodynamic relationships are well established, different geochemical codes will tend to produce similar answers if the same thermodynamic data are used. Stated differently, the results of geochemical modeling are only as good as the data that are used. The codes listed below usually employ an individualized database, customized to meet the code's formatting requirements for looking up the appropriate values. Values in the database can be modified to incorporate newer, more reliable data or to include additional data not previously available. These data commonly come from a variety of sources that may or may not be referenced. Some of the more conventional sources of these data are listed in Table D-1.

\subsubsection{SUPCRT}

The SUPCRT code (Helgeson et al., 1978; Johnson et al., 1991) was initially developed at Northwestern University to calculate equilibrium constants as a function of temperature and pressure. The thermodynamic database currently contains (Johnson et al., 1991) over 290 aqueous species, 175 minerals, and 16 gases. Using thermodynamic relationships outlined above, SUPCRT calculates $\triangle G_{R}$ (or equivalently $\log \mathrm{K}$ ) for reactions or species as a function of temperature and pressure. The total P-T range available to the code is $0-1000{ }^{\circ} \mathrm{C}$ and $1-5000$ bars, although much of the data are based on hightemperature experiments. The data have been carefully screened and checked for internal consistency (Helgeson et al., 1978; Johnson et al., 1991) and data sources are fully referenced. Since SUPCRT has been in the public domain since about 1978, extensive use in a variety of geochemical problems has resulted in an extremely robust and portable code. Written in FORTRAN 77, SUPCRT has been adapted to both UNIX- and DOS-based systems, and there is a new version available for the Apple MacIntosh (Johnson et al., 1991). Recent modifications to the code (Johnson et al., 1991) have resulted in a more streamlined code with user prompts to aid the user in constructing the input file for a given problem.

\subsubsection{PHREEQE}

The PHREEQE (pH-REdox-EQuilibrium-Equations) equilibrium speciation code, based on the WATEQ and MIX2 codes, was developed in the 1970s by the USGS (Parkhurst et al., 1980; Plummer et al., 1988). The code has been used extensively, and has been applied to a wide variety of geochemical problems. The code is able to model $\mathrm{pH}$, redox potential, equilibrium speciation, and mass transfer as a function of reaction progress. The code is also able to model the mixing of two aqueous solutions. The code has been modified by Brown et al. (1991) to allow mineral precipitation and dissolution, fixed $\mathrm{pH}$, and electrostatic adsorption processes, and Pitzer's equations for a limited number of components have been adapted to allow the calculation of activity coefficients in high-ionic-strength brines (Plummer et al., 1988). PHREEQE can also model the mixing of solutions of differing composition and the titration of one solution against another. Input is formatted, although Fleming and Plummer (1983) developed an interactive program PHRQINPT for constructing the input files. Originally, the thermodynamic database had only a limited number of minerals available (approximately 40), but has since been enlarged to include up to 200 minerals (Parkhurst et al., 1990). In addition, Tweed (1988) developed the code PICKER to select an appropriately sized database from a larger database of the same format. In addition to the database distributed with the software (Parkhurst et al., 1990), appropriately formatted databases have also 
been developed as part of the CHEMVAL project (Read and Broyd, 1991) and using the HATCHES database management system (Cross and Ewart, 1991). The PHREEQE code uses conservation of charge, conservation of electrons, total alkalinity, mass-balance, mineral equilibrium, and mass-action expressions to calculate the chemical composition of a system at equilibrium. The code iterates toward a mass balance, using back-substitution until the solution is within 20 percent of the convergence criteria, at which point a Newton-Raphson iteration scheme is used to calculate $\mathrm{pH}$, Eh, and solution composition. Mineral saturation indices are then calculated by direct substitution. PHREEQE uses the van't Hoff equation [Eq. (2-16)] to correct equilibrium constants for temperature effects, and is therefore limited to fairly low temperatures. There is also an option for a user-specified power function, of the general form $\log \mathrm{K}=\mathrm{A}$ $+\mathrm{BT}+\mathrm{C} / \mathrm{T}+\mathrm{Dlog} \mathrm{T}+\mathrm{ET}^{-2}$, for mineral equilibrium constants as a function of absolute temperature. The code is in FORTRAN, and has been adapted to VAX and IBM mainframe systems, as well as IBM personal computers.

\subsubsection{WATEQ}

The WATEQ and WATEQF series of codes were initially written in the early 1970s and have since been modified and updated (Truesdell and Jones, 1974; Plummer et al., 1976; Ball et al., 1979, 1987). The database has been expanded recently (Ball and Nordstrom, 1991) to include uranium data from the NEA database and equilibrium constants recommended in Nordstrom et al. (1990). Using tabulated equilibrium constants, the code calculates equilibrium distribution of elements in natural waters. Limited to $1 \mathrm{~atm}$, WATEQ uses the van't Hoff equation to model temperatures from $0-100^{\circ} \mathrm{C}$. The code can use either the Debye-Huickel or Davies equation to calculate activity coefficients. WATEQ has an extensive database that has been exhaustively documented and widely tested in a number of groundwater investigations. The code is limited to equilibrium chemical speciation. WATEQ was originally written in PL/1 programming language. The code has been adapted to FORTRAN in the WATEQF series. Backsubstitution is used to solve for mass balance.

\subsubsection{EQ3/6}

The EQ3/6 code, originally developed at the University of California, Berkeley, in the late 1970 s, is one of the more elaborate and complicated geochemical codes (Wolery, 1979, 1983; Wolery et al., 1990). The EQ3/6 package is composed of two programs; $E Q 3$ calculates equilibrium aqueous speciation and determines the saturation indices $(\mathrm{Q} / \mathrm{K})$ for a wide variety of minerals, while EQ6 calculates mass transfer and reaction progress for heterogeneous precipitation/dissolution. The current database is extensive, and relies mainly on the internally consistent SUPCRT database for the more common species, minerals, and gases. The database has recently been modified (Wolery et al., 1990) to include thermodynamic data for radionuclides, including uranium, plutonium, neptunium, thorium, and americium, although these data have not been checked for internal consistency. A limited number of solid-solution minerals are also available in the new database. EQ3/6 has a wide variety of options allowing the user to tailor a simulation to a wide variety of applications. Although the code is largely restricted to dilute solutions by the activity/concentration models used, there is a limited amount of data available for using the Pitzer equations in high-concentration brines (Jackson and Wolery, 1985; Jackson, 1988). There has also been some discussion on incorporating sorption models into EQ3/6 (Viani, 1988).

The reaction path modeling available for EQ6 allows the user to consider reaction kinetics, and reaction progress as a function of time is possible. However, the validity of this calculation is dependent on the validity of the kinetic rate data. The code extrapolates to higher temperatures by fitting a polynomial (up to 6th order) to temperature-log $\mathrm{K}$ data. The code is restricted to $1 \mathrm{~atm}$ at temperatures 
of $100{ }^{\circ} \mathrm{C}$ and lower. Up to $300{ }^{\circ} \mathrm{C}$, pressure follows the steam saturation curve for pure water. Temperature can be varied as a user-assigned function of the reaction progress variable $\xi$ [Eq. (D-11)]. Input is formatted and very detailed, although the most recent version (Wolery et al., 1990) has a more user-friendly interface for creating input files. The code is in FORTRAN, and has been adapted to CDC and VAX mainframe and CRAY supercomputer systems as well as Sun and Alliant workstations.

\subsubsection{MINTEQ}

The MINTEQ family of codes (Felmy et al., 1983; Brown and Allison, 1987; Allison et al., 1990) is based on the earlier MINEQL code (Westall et al., 1976) and uses the WATEQ4 database. Also an equilibrium speciation code, MINTEQ allows modeling of mineral precipitation/dissolution, ion exchange, and redox processes. Nonequilibrium kinetics cannot be modeled, but seven adsorption models are currently available. The database from WATEQ4 is well documented and extensive (Ball et al., 1987). Additional modifications to the database (Turner et al., 1993) have included the radionuclide data for U, $\mathrm{Pu}, \mathrm{Am}, \mathrm{Np}, \mathrm{Th}, \mathrm{Tc}, \mathrm{Cs}, \mathrm{Ra}, \mathrm{Ru}, \mathrm{Eu}, \mathrm{Sn}, \mathrm{Co}, \mathrm{Sr}$, and $\mathrm{Zr}$ from the EQ3/6 database. Based on a set of component (or basis) species, MINTEQ allows the user to consider a large number of aqueous species, providing $\log \mathrm{K}$ data is available. Ionic strength effects can be modeled using either the Davies (default) or Debye-Hückel equation. Much of the database is limited to calculations at $25^{\circ} \mathrm{C}$. Extrapolations to higher temperatures are accomplished through the van't Hoff equation if $\Delta H_{R}^{o}$ data are available. An alternative approach to temperature extrapolation is to construct a database consisting of polynomial functions expressing $\log \mathrm{K}$ as a function of temperature in a fashion similar to PHREEQE. Input is prepared using the preprocessor PRODEFA2 to define the problem of interest. Written in FORTRAN IV, and using a Newton-Raphson iteration scheme, the most recent version (MINTEQA2) is available through the U.S. Environmental Protection Agency (EPA) for DEC VAX/VMS systems and for the IBM personal computer.

\subsubsection{GEOCHEM}

Designed specifically for calculating equilibrium soil chemistry, the GEOCHEM code contains over 1800 equilibrium constant data for aqueous species involving over 80 elements and organic compounds (Sposito and Mattigod, 1980; Kincaid et al., 1984a,b; Sposito, 1986; Parker et al., 1987). The data is limited to $25^{\circ} \mathrm{C}$ and $1 \mathrm{~atm}$, with no options for extrapolation to higher temperatures and pressures. Elevated temperatures and pressures can only be modeled if the user modifies the $\log \mathrm{K}$ values in the current database or creates a new database to reflect the higher temperatures. Ionic strength effects are modeled using the Davies equation. The code is written in FORTRAN IV and has been adapted to IBM and VAX mainframe systems, as well as IBM personal computers. Input is either by formatted batch entry or through an interactive mode.

\subsubsection{ECHEM}

ECHEM is a proprietary code developed by the Electric Power Research Institute (EPRI) as a part of the FASTCHEM hydrogeochemical transport code (Morrey, 1988). The approach used in ECHEM is similar to that used in the MINTEQ family of codes, using basis species and equilibrium constant data to model gas-mineral-aqueous speciation, redox, precipitation/dissolution, and adsorption processes. The database is a modified and extended version of the MINTEQ database (Krupka et al., 1988). Temperature effects are modeled either using the polynomial expression from MINTEQ or the van't Hoff relationship. Therefore, the code is limited to lower temperatures, probably below $100^{\circ} \mathrm{C}$. Pressure is restricted to 
$1 \mathrm{~atm}$. Eight sorption models are included, and activity coefficients are calculated using either the Davies or Debye-Hückel equations. Although the code is written in FORTRAN, current applications are largely restricted to IBM mainframes with a networked IBM workstation configuration used by the FASTCHEM system.

\subsubsection{HYDRAQL}

HYDRAQL was developed at Stanford University (Papelis et al., 1988) as a refined and enhanced version of the MINEQL code (Westall et al., 1976). Particular attention was paid to improving the error messages associated with the code. A Newton-Raphson iteration method is used to solve the non-linear algebraic mass balance equations. Written in FORTRAN 77 for the IBM PC, the database formatting requirements of HYDRAQL are similar to MINEQL. The database is fairly complete, and can be modified and expanded to include additional data. Input is by a series of format-free batch files. A subroutine CHECK checks the input for self-consistency and calls up the error message subroutine if problems are encountered. Thermodynamic data $\left(\log \mathrm{K}, \Delta \mathrm{H}_{\mathrm{R}}^{\mathrm{O}}\right)$ are stored relative to a restricted set of components which can be combined to form every species considered in the calculations. Data are retrieved from a formatted database according to the problem definition. Five surface complexation models are available to model sorption processes. Redox processes can also be modeled if the appropriate data are available. Temperature limits are 0 to $75^{\circ} \mathrm{C}$, and extrapolation beyond $25^{\circ} \mathrm{C}$ is by the van't Hoff equation. The Davies equation is used to correct for ionic strength effects. The parameter $\mathrm{A}$ in the Davies equation [similar to Eq. (2-18) with $\mathrm{Ba}_{\mathrm{i}}=1.0$ and $\mathrm{b}_{\mathrm{i}}=0.3$ ] is corrected for temperature effects according to the parametric equation:

$$
\mathrm{A}=0.50886\left[8 \times 10^{-4}(\mathrm{~T}-25)\right]+10^{-5}(\mathrm{~T}-25)^{2}
$$

where

$$
\mathrm{T}=\text { Temperature of interest }\left({ }^{\circ} \mathrm{C}\right) \text {. }
$$

\subsubsection{The Geochemist's Workbench}

The Geochemist's Workbench (Bethke, 1992) is a proprietary code designed to calculate chemical equilibria, stability diagrams, reaction progress, and plot the results of the calculations. The code contains four geochemical programs. RXN balances chemical reactions and handles the mass action/mass balance calculations. RXN will also calculate the temperature at which a given reaction is in equilibrium. ACT2 and TACT calculate fugacity-activity and temperature-activity-fugacity diagrams, respectively. REACT calculates reaction paths for both reversible and irreversible reactions in gas/water/solid systems under either isothermal or nonisothermal conditions. REACT is also able to model the fractionation of stable isotopes, buffered systems, and kinetic precipitation/dissolution if rate constant data are available. The postprocessor program GTPLOT is used to plot reaction progress results calculated by REACT. Thermodynamic data included with the code are based largely on the SUPCRT data set (Johnson et al., 1991). Like $\mathrm{EQ} 3 / 6$, data are constrained to $1 \mathrm{~atm}$ for temperatures to $100^{\circ} \mathrm{C}$, and the steam-saturation curve for temperatures from $100^{\circ}$ to $300{ }^{\circ} \mathrm{C}$. For extrapolation to temperatures beyond $25^{\circ} \mathrm{C}$, a polynomial is fit to the temperature-equilibrium constant data. Ionic strength activity corrections are made using the Debye-Hückel equation. For a limited dataset, however, applications are available that use either the Pitzer equations (Pitzer, 1973, 1979) or the Harvie-Møller-Weare model (Harvie et al., 1984) for concentrated solutions. Designed for Unix workstations, input into the different programs is interactive 
through either a series of commands or menus. The code also supports output to a number of commonly used printers and monitors. The Geochemist's Workbench is copyrighted, and licensing is available through Craig Bethke and the University of Illinois.

\subsubsection{Summary and Conclusions}

The equations outlining the effect of temperature on equilibrium and kinetic chemistry were presented. Geochemical codes that are commonly used to address thermochemical problems were described. These codes generally use either free-energy minimization or mass action/mass balance considerations to solve for chemical equilibria. Code history, methods, and limitations were also discussed. At present, only the EQ3/6, PHREEQE, and Geochemist's Workbench codes are able to model reaction progress, and only EQ3/6 and Geochemist Workbench can use reaction kinetics to model system evolution as a function of time. As discussed in Appendix $\mathrm{D}$, however, these results are only valid if appropriate rate constant data are available. Several of the codes use the van't Hoff equation [Eq. (2-16)] relation to módel $\log \mathrm{K}$-temperature relationships, and are therefore limited to relatively low temperatures $\left(<100^{\circ} \mathrm{C}\right)$. Some temperature predictions for the near-field exceed the likely limits of the van't Hoff equation (Appendix A), and curve-fitting of log K-temperature data may be necessary to extrapolate. However, if a heat-pipe is established, temperature increases may be limited to the boiling temperature of the solution in parts of the system (see Section 2.2.1 and Pruess et al., 1990a). In addition, many of the thermodynamic data, especially for many of the key radionuclides, are only available for low temperatures. Most of the codes use either the Davies or Debye-Hückel relations to correct activity coefficients for ionic strength and are therefore limited to relatively dilute solutions. EQ3/6 and PHREEQE, however, have been modified to use the Pitzer equations to model high-ionic-strength brines. Although this may be necessary for localized areas of crevice corrosion, most water compositions reported for the Yucca Mountain vicinity are relatively low-ionic-strength solutions (e.g., Kerrisk, 1987). All of the codes are available in FORTRAN, and most are adapted for use on commonly-available mainframe and/or personal computers. It is clear that the coupled effects of temperature on system chemistry are significant and must be considered.

\subsection{CHEMICAL $\Rightarrow$ THERMAL}

Chemical processes primarily affect the temperature field (and therefore thermal processes) through: (i) changes in material properties (e.g., thermal conductivity, heat capacity), and (ii) the production (or consumption) of heat through exothermic (or endothermic) chemical reactions.

\subsubsection{Material Properties}

Chemical processes may change the heat capacity or the thermal conductivity of a porous medium through chemical alteration or dissolution/precipitation of the rock mineral assemblages. An example of a chemical alteration is the hydrolysis of feldspars, which can lead to the production of clays which have different thermal properties than the precursor minerals. The formation of clays would affect the thermal conductivity of the medium, which could influence the conductive heat transfer.

However, the differences in conductivity of different minerals are generally small, and are treated as constant values in many analyses (Lewis, 1990). Under anticipated conditions at Yucca Mountain, variation in thermal properties due to chemical processes are likely to be of minimal importance relative to natural variability. The reader is referred to the final portion of Section 2.2 .4 for a comparison between 
variations due to hydrologic processes and variations due to inherent and spatial uncertainties of the thermal conductivity at Yucca Mountain. Similarly, there is little to no evidence that suggests chemical processes will affect the thermal conductivity more than what is already recognized in variations between rock-sample measurements and variations between different rock units.

\subsubsection{Exothermic or Endothermic Reactions}

Dissolution/precipitation reactions during the hydrothermal alteration of rock may provide an additional source/sink to the overall heat balance for a given system. Norton and Cathles (1979) note that, except for quartz, the hydrolysis of most silicate and oxide minerals exhibits a negative enthalpy of reaction $\left(\Delta \mathrm{H}_{\mathrm{R}}\right)$, indicating a heat-producing exothermic reaction. This can be quite large, on the order of $10^{3}-10^{4} \mathrm{~J} / \mathrm{mol}$, and decreases with increasing temperature of reaction. Exothermic oxidation of sulfur and oxygen aqueous species are on the same order. In contrast, hydrolysis of quartz and sulfide minerals have positive enthalpies of reaction, which act as heat sinks of a similar magnitude. The mass balance analysis of Norton and Cathles (1979) examined the alteration of a quartz diorite to two separate mineral assemblages, quartz + sericite and $\mathrm{K}$-feldspar + biotite at $300^{\circ} \mathrm{C}$, assuming that the heats of reaction for reversible and irreversible dissolution are equal and that the effects of aqueous dissociation reactions were minor. Total energy released due to exothermic hydrolysis reactions ranged from 185 to $285 \mathrm{~J} / \mathrm{g}$. Ellis and Mahon (1977) estimate that devitrification of volcanic glasses could release up to $335 \mathrm{~J} / \mathrm{g}$ due to exothermic reactions. Norton and Cathles (1979) conclude that hydrolysis would extend the duration of a thermal perturbation, and that hydrolysis reactions can account for about 15 percent of the total heat content of an igneous pluton crystallizing from liquidus temperatures on the order of $700-800{ }^{\circ} \mathrm{C}$. The degree to which this would perturb the temperature field would depend on the rate of reaction and the rate at which heat is transferred out of the altered rock.

Parry et al. (1980) also investigated the contribution of hydrothermal alteration on the heat flow in the geothermal system at Roosevelt Hot Springs, Utah. Exothermic alteration of feldspars to clays, and the oxidation of sulfur species, were modeled as a function of alteration intensity. Exothermic reactions could have contributed as little as $30 \mathrm{~J} / \mathrm{cm}^{3}$ for fresh rock and as much as $7100 \mathrm{~J} / \mathrm{cm}^{3}$ for the most intensely altered rocks. However, measured and calculated temperatures profiles indicated that the contribution of alteration reactions measured in 1980, heat flow was insignificant relative to the convective heat transport.

\subsubsection{Computer Codes}

Computer codes typically do not model only $\mathrm{C} \Rightarrow \mathrm{T}$ coupled processes. Many of the codes that model $\mathrm{T} \Rightarrow \mathrm{C}$ coupled processes can model $\mathrm{C} \Rightarrow \mathrm{T}$. These computer codes are discussed in Section 2.5.3, and the discussion is not repeated here.

\subsubsection{Summary and Conclusions}

In this section, the coupling mechanisms by which chemical processes influence thermal processes were reviewed. Namely, (i) changes in material properties, and (ii) exothermic or endothermic chemical reactions were discussed. Although both coupling mechanisms are plausible, there appears to be little evidence to suggest they will be important at Yucca Mountain. Overall, the $C \Rightarrow T$ coupling appears to be negligible in comparison with natural variability in material properties and thermal loads of the emplaced HLW. 


\section{$2.7 \quad$ HYDROLOGIC $\Rightarrow$ MECHANICAL}

The state of stress in a rock mass is coupled to groundwater flow. This coupling is present in both directions, as even small changes in the fracture openings cause large changes in permeability, and changes in permeability will in turn affect the groundwater flow, and thereby the pressure acting on the rock. In applications where the fluid is pumped into the fractures or boreholes at high pressures, as in enhanced oil recovery and geothermal energy production, the hydrologic conditions strongly influence the mechanical state, as new fractures are created or existing fractures are propped open to enhance the permeability. However, in applications such as underground disposal of HLW, the fluid pressures in fractures are expected to be primarily a result of gravity. If one assumes that these fractures would be fully saturated, the resulting fluid pressures would correspond to the height of the water column in the fracture. Since these pressures are small compared to the in situ state of stress within the rock, the $\mathrm{H} \Rightarrow \mathrm{M}$ coupling is expected to be much less important than the $\mathrm{M} \Rightarrow \mathrm{H}$ coupling.

\subsubsection{Mechanical Properties}

Due to the partially-saturated nature of the tuff at the proposed repository site at Yucca Mountain, and that only periodic flow is likely to occur in the fractures, fluid-induced changes in rock properties may not be important. Laboratory experiments have shown that rocks are generally weaker when saturated with water. Olsson and Jones (1980) show that, for the Grouse Canyon tuff, a volcanic rock unit located at the Nevada Test Site, saturated samples are approximately 24 percent weaker than dry samples in unconfined compression. No literature was found on the experimental testing of strength properties in partiallysaturated rocks, more representative of conditions at Yucca Mountain, where one would expect the impact of hydrologic conditions on mechanical properties to be much less. Wada and Takahashi (1990) also obtained results that indicated a significant change in the Young's modulus of granitic rocks when subjected to a pressurized high-temperature water environment up to $350^{\circ} \mathrm{C}$. Jaeger and Cook (1979) reported that the introduction of water increased the coefficient of friction for some minerals and decreased it for other minerals. However, more experimental work is needed on various types of rocks to determine whether water on the joint or fracture surfaces will significantly lubricate them to cause a reduction in joint shear strength.

Unanticipated (i.e., low probability) processes and events such as large earthquakes, as well as anticipated underground nuclear explosion testing at the nearby Nevada Test Site, etc., could conceivably have a higher impact on the $\mathrm{H} \Rightarrow \mathrm{M}$ response of a fractured rock mass. For instance, such an event could suddenly cause an increase in the fluid pressure within a joint. If the joint or fault was under a shear stress, the fluid pressure could reduce the effective stress across such a feature to such an extent that mechanical slip would occur. However, due to the current depth of the water table below Yucca Mountain $(>200 \mathrm{~m})$, this is expected to have a negligible impact on repository performance.

\subsubsection{Buffer Swelling}

It is expected that, during closure of the repository, excavations (e.g., ramps and emplacement tunnels) will be backfilled. The backfill may contain some proportion of bentonite, which will create swelling pressure when saturated with water. Swelling pressures of $10 \mathrm{MPa}$ were measured in the field for the Stripa Project for an almost completely-saturated buffer material. However, the swelling depends on the final density of the buffer material, and would be expected to vary from 3 to $15 \mathrm{MPa}$ (Carlsson et al., 1989). Within an emplacement borehole, for instance, the swelling bentonite creates internal pressure 
in the filling, which acts as a compressive stress in the normal direction toward the excavation walls. The swelling pressure, therefore, supports the excavation walls and relieves some of the compressive stresses in the circumferential direction to the excavation walls. However, if these circumferential stresses become tensile, there is a risk of tensile failure and the creation of new fractures in the radial direction, occurring even many years after completion of the backfilling. The average uniaxial compressive and Brazilian tensile strengths of the Topopah Spring member of the Paintbrush tuff have been measured to be $95.9 \mathrm{MPa}$ and $12.8 \mathrm{MPa}$, respectively, so that swelling-induced cracking would be possible, depending on the magnitude of in situ and excavation-induced compressive stresses around the openings. However, current design for the waste packages at Yucca Mountain calls for a small air gap between the waste container and borehole wall. In addition, there appears to be little interest in using clay backfills at Yucca Mountain. For these reasons, the $\mathrm{H} \Rightarrow \mathrm{M}$ coupling is not considered important for the proposed repository without clay backfills.

\subsubsection{Computer Codes}

Computer codes that can be used for modeling hydrologic and mechanical processes include FLAC, UDEC, ROCMAS, and FEHMS. These computer codes are briefly discussed in Sections 2.3 .3 and 3.1.2, and the discussion is not repeated here.

\subsubsection{Summary and Conclusions}

In this section, the impact of $\mathrm{H} \Rightarrow \mathrm{M}$ coupling has been discussed. It is expected that, for the Yucca Mountain repository site, pore pressures generated within the rock matrix, as well as fluid pressures within fractures, will have a negligible impact on the mechanical state of the rock. This is primarily due to the low matrix compressibility (i.e., matrix porosity is a weak function of stress) and the partiallysaturated nature of the site.

Swelling of buffer/backfill materials containing bentonite clay can create an $\mathrm{H} \Rightarrow \mathrm{M}$ response, depending on the percentage of bentonite and degree of saturation. Some swelling is beneficial in supporting the rock around the excavations, and also in creating a good seal. However, too much swelling can generate large tensile stresses within the rock, leading to fracturing.

Finally, it was emphasized that material properties can be influenced by the presence of moisture within the rock. Mechanical property testing conducted to date has dealt only with fully-saturated rock specimens. For the Yucca Mountain welded tuff, these property tests would represent a lower bound on compressive strength.

\section{8 $\quad$ MECHANICAL $\Rightarrow$ HYDROLOGICAL}

Mechanical processes can affect hydrologic processes primarily through fracture apertures which affect (i) effective hydraulic conductivity, and (ii) seismic pumping. For the tuffaceous rock at Yucca Mountain, the matrix is essentially incompressible for hydrologic considerations. Hence, in this section, only the changes in fracture aperture are considered as mechanisms for a $\mathrm{M} \Rightarrow \mathrm{H}$ coupling. 


\subsubsection{Effective Hydraulic Conductivity}

The normal stresses across a fracture are expected to influence the fracture aperture. Increasing the compressive stress acts to close the fracture aperture, while reducing the compressive stress acts to increase the fracture aperture. Also, natural rock joints or fractures exhibit some degree of roughness such that shearing of the joint causes dilation, which increases the fracture hydraulic aperture. Shearing under low normal stresses causes the largest dilation, as the joint rides up on the asperities.

It is theoretically convenient (for hydrologic purposes) to idealize fractures as being void regions between two parallel surfaces. For such an idealized fracture, it has been shown that small changes in the aperture can significantly change hydrologic flow rates and the flow field (Witherspoon et al., 1979). For a saturated fracture under a constant hydraulic head gradient, the total flow rate is approximately proportional to the hydraulic aperture cubed. Hence, in a fractured medium it may be important to understand mechanical processes to predict hydraulic behavior.

Rutqvist et al. (1991) analyzed the changes in hydraulic conductivity due to excavation-induced stresses. Around a circular excavation, three orientations of fractures were considered: circumferential, radial, and axial, as shown in Figure 2-22. For discussion purposes, radial fractures could act as a conduit for condensate drainage (see Sections 2.1.4 and 2.2.3) into or out of an excavation. Similarly, circumferential fractures can act to divert condensate drainage around an excavation. Rutqvist et al. (1991) note that excavation-induced stresses will tend to (i) decrease the aperture for radial fractures, (ii) increase the aperture for circumferential fractures, and (iii) increase the aperture for axial fractures. The first two results can be understood with the aid of Figure 2-23.

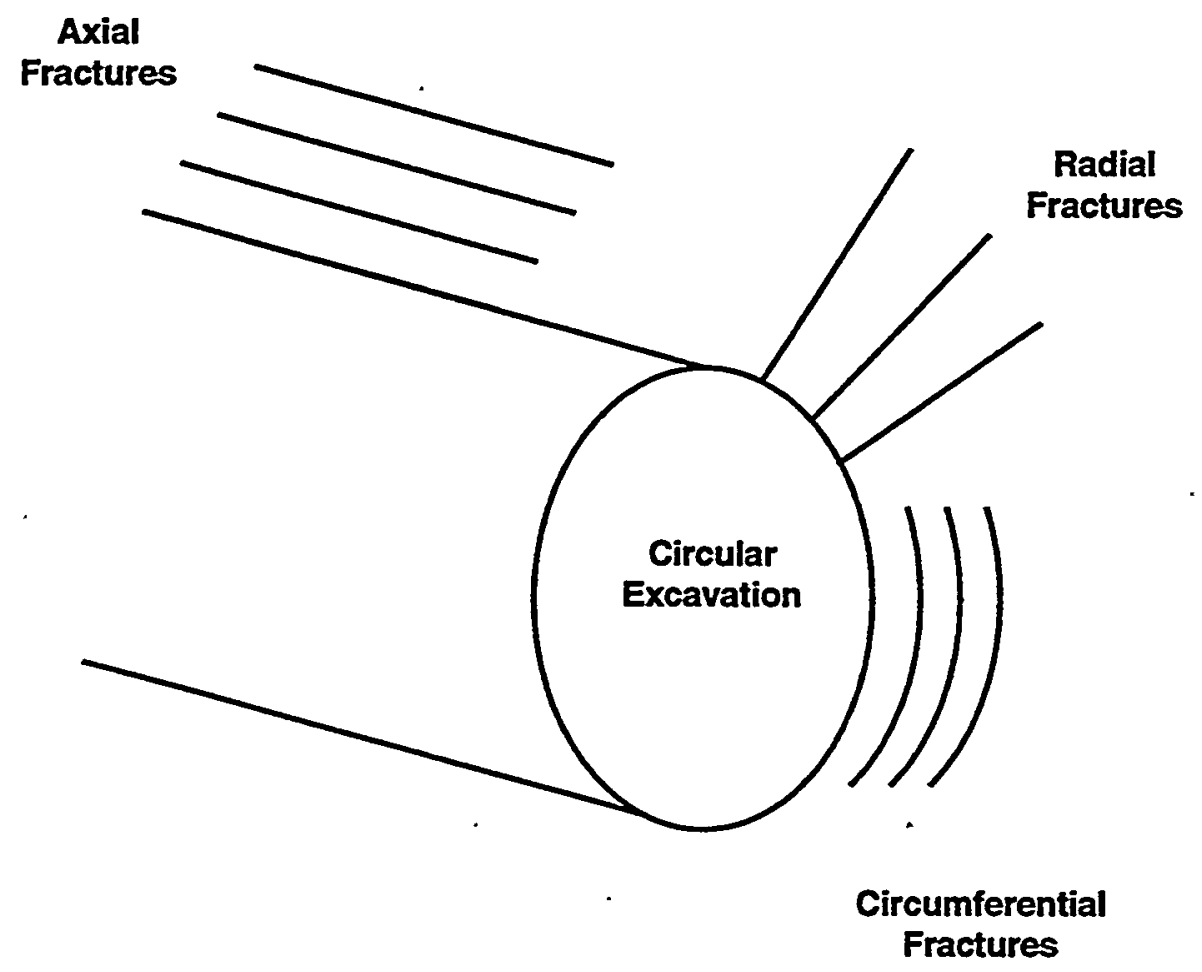

Figure 2-22. Ilustration of axial, radial, and circumferential fractures around a circular excavation. 


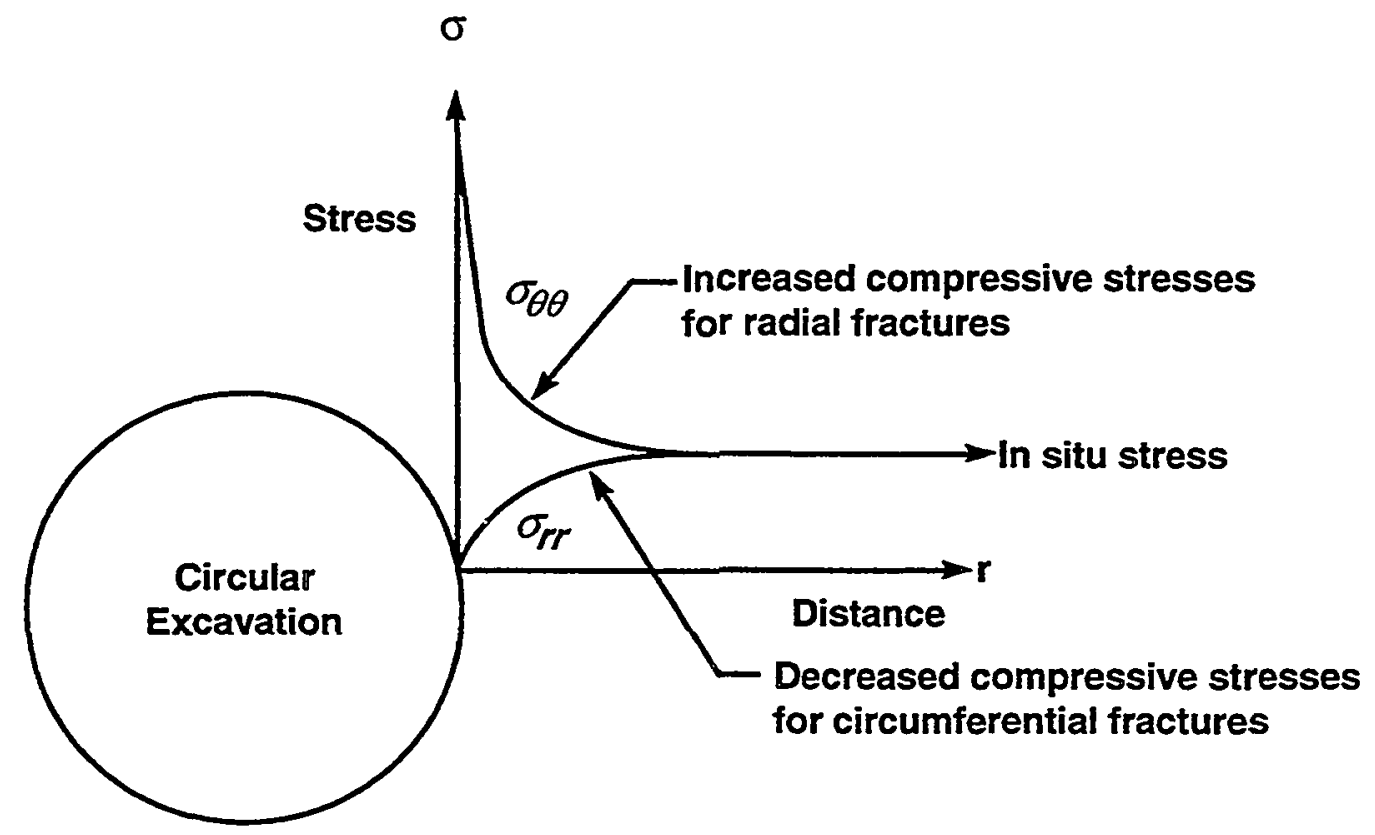

Figure 2-23. Ilustration of normal stresses in the circumferential $\left(\sigma_{\theta \theta}\right)$ and radial $\left(\sigma_{\mathrm{rr}}\right)$ orientation

The circular excavation can be viewed as existing in a 2D, isotropic and homogeneous medium for which the conditions of plane strain can be applied. The normal stresses in the circumferential and radial orientations vary near the excavation, as shown in Figure 2-23. The circumferential stress increases dramatically near the excavation surface, and this tends to close radial fractures. For a linear, isotropic, homogeneous rock mass medium in a hydrostatic stress field, the maximum circumferential compressive stress is two times greater at the surface of a circular excavation (see Brady and Brown, 1985). The radial compressive stress decreases near the excavation and is zero at the excavation surface. The decreased radial stress tends to open circumferential fractures near the excavation. Both of these effects decrease as the distance from the excavation into the medium is increased. These results have been deduced by Kelsall et al. (1984), Pusch (1989), Rutqvist et al. (1991), among others. The increase in axial hydraulic conductivity has been experimentally observed by Pusch (1989). However, this result is not obvious from the simple $2 \mathrm{D}$ plane strain model. In most cases, the stress field is not hydrostatic, and the rock mass has some degree of anisotropy or inhomogeneities. As a result, the circumferential and radial stresses will not equal each other at a distance from the excavation boundary, as depicted in Figure 2-23. A trend similar to that shown in Figure 2-23 can be shown for the normal stresses in the circumferential and radial direction in the case of nonhydrostatic in situ stress states.

The response of a rock mass to stress changes around an underground opening may result in the formation of an inelastic or "plastic" zone adjacent to the opening, depending on the properties of the rock mass and the magnitudes of the induced stresses. The distinction between the elastic and plastic zones around an underground opening is important with respect to stress distributions and the resultant effects on fracture permeability. As mentioned above in the elastic case, it is expected that the permeability of circumferential fractures should be increased, whereas the permeability of radial fractures should be 
reduced. However, in the case of plastic deformations adjacent to an opening, both the radial and circumferential stresses are reduced close to the wall in the plastic zone, so that the permeability of both tangential and radial fractures should be increased in this localized area (Kelsall et al., 1984). The impact of this localized zone of increased permeability around the excavation is uncertain since the high stress concentrations would shift outward and consequently decrease joint apertures in this still elastic region. The whole plastic zone may be treated as part of the opening. The depth (stress level) at which inelastic or plastic behavior is first observed depends, in general, on the rock mass strength. Hoek and Brown (1980) provide an analytical solution to estimate the depths in a circular shaft at which the plastic and fracture zones will be first observed for given rock conditions. At the proposed repository horizon, which is approximately $300 \mathrm{~m}$ below the surface, the inelastic zone around the excavations is expected to be small due to the strong nature of the Topopah Spring welded tuff and the relatively shallow depths. However, excavations into the weaker Calico Hills unit during site characterization studies may produce larger inelastic regions around the excavations, and consequently larger increases in permeability.

\subsubsection{Seismic Pumping}

The potential for large changes in the water table elevation as a result of seismic activity has been a consideration at Yucca Mountain (Archambeau and Price, 1991; Powers et al., 1991; Carrigan et al., 1991; National Research Council, 1992). It has been proposed that the water table at Yucca Mountain has periodically risen above the level proposed for a repository. The changes in water table elevation were proposed to be due to nearby earthquakes, hence an $M \Rightarrow H$ coupling known as "seismic pumping." In seismic pumping, seismic events compress the ground, which result in reduced available storage volume for the liquid water. The liquid flows to increase the elevation of the water table. Szymanski proposed that tectonic cycles characterized by slow extensional deformation, and punctuated by seismic events, inducted large upwellings of the water table.

The scenario of seismic pumping at Yucca Mountain has been reviewed by Archambeau and Price (1991), Powers et al. (1991), Carrigan et al. (1991), the National Research Council (1992), among others. Probably the most extensive review of seismic pumping at Yucca Mountain has been conducted by the National Research Council (1992) which concluded that seismic pumping is inadequate to raise the water table significantly at Yucca Mountain.

Carrigan et al. (1991) conducted numerical analyses investigating the potential for water table excursions induced by seismic events at Yucca Mountain. The simulations employed a 2D elastic boundary element model of the Earth's crust to determine the pore pressure distribution following a seismic event. This pore pressure distribution was then used in an initial-value hydrologic computational model that tracks the water table as a function of time in a $2 \mathrm{D}$ hydrologic domain. The relation used between the pore pressure and volumetric strain may be derived as

$$
P_{P}=\frac{-E}{3}\left[\frac{1}{1-2 v_{P}}\right]\left(e_{x x}+e_{y y}\right)
$$

where

$$
\begin{array}{ll}
\mathrm{P}_{\mathrm{P}} & =\text { pore pressure }\left(\mathrm{N} / \mathrm{m}^{2}\right), \\
\mathrm{E} & =\text { Young's modulus }\left(\mathrm{N} / \mathrm{m}^{2}\right), \text { and } \\
\mathrm{e}_{\mathrm{ij}} & =\text { components of strain (dimensionless). }
\end{array}
$$




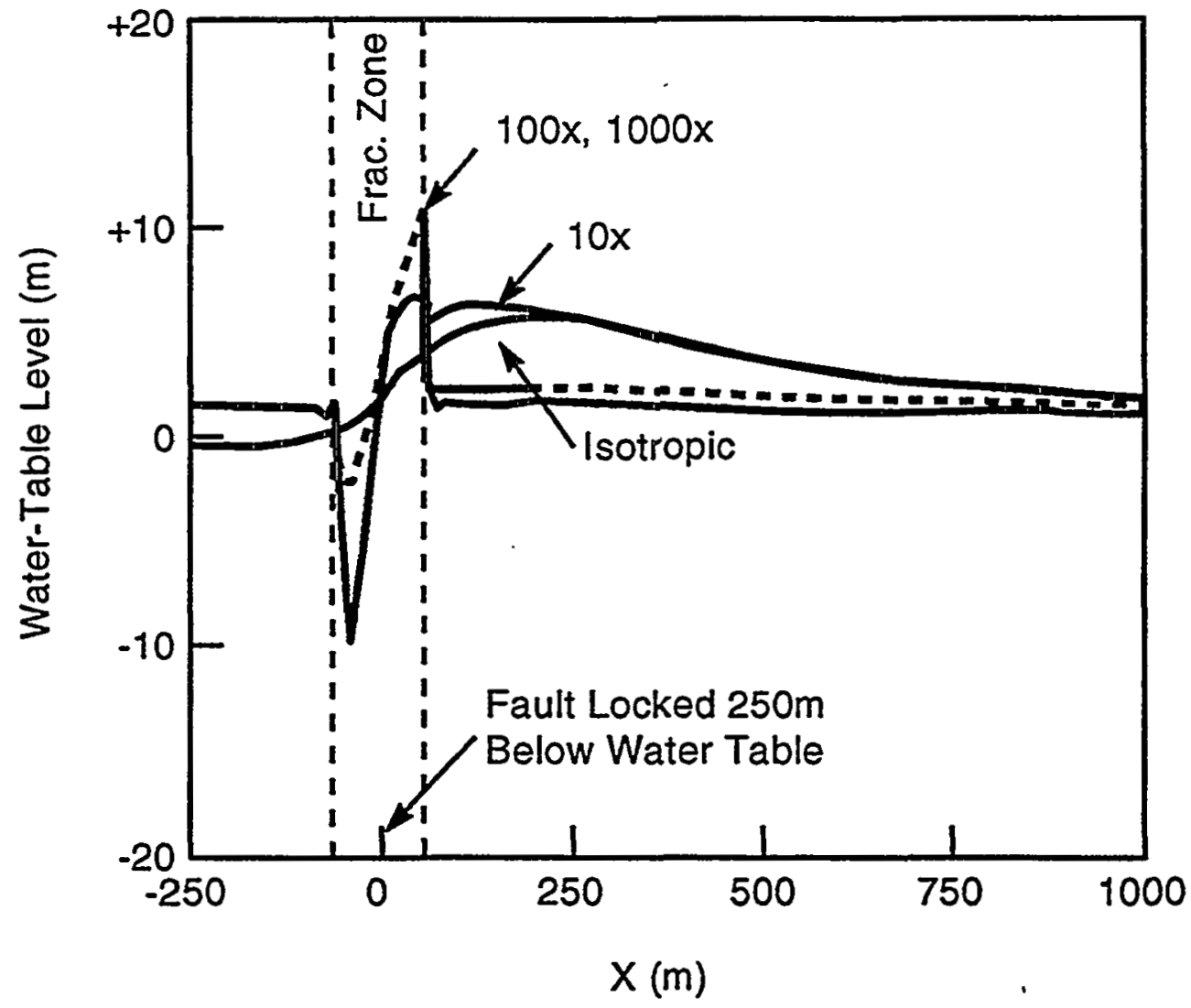

Figure 2-24. Water table response for isotropic permeability and for local vertical permeability enhancement $\left(10\right.$ to $10^{3}$ ) within a 100 -m-wide anisotropic region centered on a normal fault simulating a zone of vertical fracturing. Results are based on a 1-m slip allowed on a fault located $250 \mathrm{~m}$ below the water table ( Carrigan et al., 1991).

Figure 2-24 shows the predicted maximum rise in the water table below Yucca Mountain (approximately $12 \mathrm{~m}$ ) due to a $1-\mathrm{m}$ slip on a vertical fault locked $750 \mathrm{~m}$ below the surface $(250 \mathrm{~m}$ below the water table). This particular geometry, in which the fault was locked some distance below the ground surface, produced the largest strains and consequently largest pore pressures when slip was allowed to occur. The results in Figure 2-24 represent water table rises as a result of enhancing the vertical permeability with a 100 -m-wide region centered on the normal fault to simulate a zone of vertical fracturing. Much smaller water table rises occurred when no vertical fracturing was simulated. Since the current repository horizon at Yucca Mountain is $200-300 \mathrm{~m}$ above the water table, these results suggest that the impact of seismic pumping is negligible. O'Brien and Tucci (1992) report a similar negligible impact of earthquakes on the water table rise at Yucca Mountain, Nevada. They found a maximum water table rise of $0.9 \mathrm{~m}$ from wells instrumented near the repository site due to the 7.5 magnitude earthquake near Landers, California on June 28, 1992. Similarly, a 5.6 magnitude earthquake occurring on June 29, 1992 at Little Skull Mountain, Nevada, approximately $30 \mathrm{~km}$ from Yucca Mountain, caused an estimated maximum water-level fluctuations of only $0.4 \mathrm{~m}$ and an estimated maximum fluid-pressure fluctuation of $1.1 \mathrm{~m}$. 
It is more conceivable for the Yucca Mountain area to experience more small-scale repetitive seismic events. Such seismic events, if repeated over a long period of time (i.e., 10,000 yrs), could conceivably create preferential water pathways, which would connect the emplacement area with a nearby perched water zone.

\subsubsection{Computer Codes}

Computer codes that can be used for modeling hydrologic and mechanical processes include ADINA, ANSYS, FLAC, UDEC, ROCMAS, and FEHMS. These computer codes are briefly discussed in Sections 2.3.3 and 3.1.2.

\subsubsection{Summary and Conclusions}

In this section, mechanical to hydrologic $(M \Rightarrow H)$ coupling mechanisms have been reviewed. The primary coupling can be related to changes in fracture apertures, since the rock matrix at Yucca Mountain is essentially incompressible for hydrologic considerations. Fracture apertures decrease for increased compressive stresses, and increase for decreased compressive stresses. The fracture apertures influence the effective hydraulic conductivity and storativity.

Near mined openings, the induced excavation and thermal loads in the surrounding rock are expected to influence fracture apertures. In certain stress states, circular excavations pinch fractures directed into the excavation and open fractures circumferentially around an excavation. This tends to direct more flow around an excavation versus into an excavation. The practical application of this coupling, however, may be insignificant, due to large uncertainties in fracture apertures, locations, and conductivities.

A second $\mathrm{M} \Rightarrow \mathrm{H}$ coupling is described as seismic pumping, which is related to gradual increases in liquid storativity, coupled with rapid decreases in storativity, due to nearby seismic events. The storativity is closely related to the fracture apertures which decrease for increased compressive loads. The liquid is forced upwards, thereby raising the water table. Although seismic pumping has recently received much attention in the literature, it appears to be negligible at Yucca Mountain.

In summary, although plausible $\mathrm{M} \Rightarrow \mathrm{H}$ coupling mechanisms exist and have been discussed in the literature, the occurrence and significance of such couplings appear to be negligible at Yucca Mountain. Overall, the $\mathrm{M} \Rightarrow \mathrm{H}$ coupling is considered negligible for the unsaturated repository, except possibly mechanically-induced changes in effective hydrologic conductivity near excavations.

\subsection{HYDROLOGIC $\Rightarrow$ CHEMICAL}

In the proposed repository environment, the potential for transport of radionuclides from the engineered barrier system through the geologic setting to the accessible environment is of chief concern in the performance of the proposed HLW repository. The primary method of radionuclide transport is anticipated to be as dissolved species in the liquid phase or, in the case of ${ }^{14} \mathrm{C}$ and possibly ${ }^{129} \mathrm{I}$, as a gas in the vapor phase (DOE, 1988). Therefore, the coupling between the hydrologic system and the chemical system is of critical importance. The following is a summary of the various approaches used to model hydrologic and chemical coupling. 


\subsubsection{Equilibrium Solute Transport}

In the absence of advective transport, chemical species are transported in the liquid phase in the direction of their concentration gradients (Freeze and Cherry, 1979). Referred to as molecular diffusion, this process is generally expressed (for a single solute in one dimension) using Fick's first law

$$
F_{m}=-D_{m} \frac{d_{w}}{d x}
$$

where

$$
\begin{array}{ll}
\mathrm{F}_{\mathrm{m}} & =\text { mass flux }\left[\mathrm{kg} \text { solute } /\left(\mathrm{m}^{2}-\mathrm{s}\right)\right] \\
\mathrm{D}_{\mathrm{m}} & =\text { molecular diffusion coefficient }\left(\mathrm{m}^{2} / \mathrm{s}\right) \\
\mathrm{C}_{\mathrm{w}} & =\text { solute concentration }\left(\mathrm{kg} / \mathrm{m}^{3}\right), \text { and } \\
\mathrm{x} & =\text { direction of transport }(\mathrm{m}) .
\end{array}
$$

The concentration gradient, $\mathrm{dC}_{\mathrm{w}} / \mathrm{dx}$, is a negative quantity in the direction of transport, indicating that species migrate from areas of high to low concentration. The molecular diffusion coefficient, $D_{m}$, is determined experimentally for the individual ionic species under consideration. For major electrolytes in groundwater (e.g., $\mathrm{Na}^{+}, \mathrm{Ca}^{+}, \mathrm{Cl}^{-}, \mathrm{HCO}_{3}{ }^{-}$), $\mathrm{D}_{\mathrm{m}}$ is in the range of $10^{-9} \mathrm{~m}^{2} / \mathrm{s}$ at $25^{\circ} \mathrm{C}$ (Freeze and Cherry, 1979; Oelkers and Helgeson, 1988; Domenico and Schwarz, 1990), increasing with increasing temperature due to the higher kinetic activity of the electrolyte species (Oelkers and Helgeson, 1988). In porous media, the apparent diffusion coefficients, $D_{\mathrm{m}}^{*}$, tend to be smaller (generally ranging from 1 to 50 percent of the diffusion coefficient) due to the more tortuous nature of the transport path (Freeze and Cherry, 1979). Fick's second law

$$
\frac{\partial C_{w}}{\partial t}=\frac{\partial}{\partial x}\left[D_{m}^{*} \frac{\partial C_{w}}{\partial x}\right]
$$

can be used to describe molecular diffusion through porous media in space and time.

Assuming that solute is only transported in the liquid phase, the classical deterministic convection-dispersion equation has been used in many studies to model nonreactive solute transport (e.g., Nielsen et al., 1986; Selim et al., 1990). For reactive solute transport in one dimension, the general equation is modified to account for the effects of water/rock interaction such that

$$
\frac{\partial}{\partial t}\left(C_{w}+\frac{(1-\phi)}{\theta} \rho C_{s}\right)=\frac{\partial}{\partial x}\left(D \frac{\partial C_{w}}{\partial x}-v C_{w}\right)+\Phi
$$

where

$$
\begin{array}{ll}
\mathrm{C}_{\mathrm{w}} & =\text { solute concentration in the liquid }(\mathrm{kg} / \mathrm{l}) \\
\phi, \theta & =\text { porosity and volumetric water content, respectively, of the medium }\left(\mathrm{m}^{3} / \mathrm{m}^{3}\right) \\
\mathrm{D} & =\text { hydrodynamic dispersion coefficient }\left(\mathrm{m}^{2} / \mathrm{s}\right) \\
\mathrm{v} & =\text { mean pore velocity }(\mathrm{m} / \mathrm{s}) \\
\mathrm{C}_{\mathrm{s}} & =\text { solute concentration }(\mathrm{kg} \text { solute } / \mathrm{kg} \text { solid) adsorbed on the solid phase, } \\
\rho & =\text { mean density of the medium }\left(\mathrm{kg} / \mathrm{m}^{3}\right), \text { and }
\end{array}
$$



diffusion, precipitation/dissolution, and radioactive decay (Nielsen et al., 1986).

The hydrodynamic dispersion coefficient can be expressed for groundwater flow in terms of the apparent molecular diffusion $D_{m}^{*}$ coefficient, mean pore velocity $v$, and dispersivity $\alpha_{D}(m)$ such that $\mathrm{D}=\alpha_{\mathrm{D}} \mathrm{v}+\mathrm{D}_{\mathrm{m}}^{*}$. In systems where the Peclet number $\left(\mathrm{Pe}=\mathrm{vx} / \mathrm{D}_{\mathrm{m}}^{*}\right)$ is less than about one, molecular diffusion is predominant. Therefore, at low velocities such as those encountered in the unsaturated zone, molecular diffusion may control solute transport (Bear and Verruijt, 1987). Equation (2-27) is frequently simplified by substituting the dry bulk density $\rho_{b}=(1-\phi) \rho$ and fluid flux $q=\theta v$ for $\rho$ and $v$, respectively.

For steady-state flow conditions, assuming that the medium is homogeneous and incompressible with respect to material properties, $\phi, \rho, v$, and $\mathrm{D}$ can be treated as constants. If equilibrium sorption is the only attenuation mechanism other than dispersion considered, $\Phi=0$, then Eq. (2-27) simplifies to

$$
\frac{\partial}{\partial t}\left[C_{w}+\frac{(1-\phi)}{\theta} \rho C_{s}\right]=\left(D \frac{\partial^{2} C_{w}}{\partial x^{2}}-v \frac{\partial C_{w}}{\partial x}\right)
$$

and $\partial \mathrm{C}_{\mathrm{s}} / \partial \mathrm{t}$ is generally provided by a sorption model (Domenico and Schwarz, 1990). For the simplest case of a linear sorption isotherm assuming instantaneous equilibrium, $\mathrm{C}_{\mathrm{s}}=\mathrm{K}_{\mathrm{d}} \mathrm{C}_{\mathrm{w}}$. Under these conditions, Eq. (2-28) simplifies further such that

$$
\frac{\partial}{\partial t}\left[C_{w}\left(1+\frac{(1-\phi)}{\theta} \rho K_{d}\right)\right]=\left(D \frac{\partial^{2} C_{w}}{\partial x^{2}}-v \frac{\partial C_{w}}{\partial x}\right)
$$

where

$$
\mathrm{K}_{\mathrm{d}}=\text { linear sorption coefficient }(1 / \mathrm{kg}) \text {. }
$$

Assuming that a linear sorption isotherm is valid, a retardation factor $R_{f}$ can be defined to reflect the attenuation of solute transport relative to water velocity (or to the transport of a non-reactive solute), such that simplifying Eq. (2-29) gives

$$
\begin{aligned}
\mathrm{R}_{\mathrm{f}} & =\left[1+\frac{(1-\phi)}{\theta} \rho \mathrm{K}_{\mathrm{d}}\right] \\
\frac{\partial}{\partial t} \mathrm{R}_{\mathrm{f}} \mathrm{C}_{\mathrm{w}} & =\left(\mathrm{D} \frac{\partial^{2} \mathrm{C}_{\mathrm{w}}}{\partial \mathrm{x}^{2}}-\mathrm{v} \frac{\partial \mathrm{C}_{\mathrm{w}}}{\partial \mathrm{x}}\right)
\end{aligned}
$$

It is stressed that the treatment of retardation $\left(R_{f}\right)$ developed in Eqs. (2-28) through (2-31) is subject to the validity of the linear isotherm and all of the assumptions and limitations inherent in the $K_{d}$ approach (Davis and Kent, 1990; Turner, 1991b). In addition to instantaneous sorption, however, several mechanisms can contribute to the retardation of solute transport, including precipitation/dissolution, matrix 
diffusion, fluid mixing, and radioactive decay. Under these conditions, the $\mathrm{K}_{\mathrm{d}}$ approach is not valid, $\Phi \neq 0$, and retardation behaves in a much more complex manner than can be described using the simple $R_{f}$ approach outlined above (McKinley and Alexander, 1992). A complex coupling of geochemical relations with transport equations is then required to account for the various mechanisms controlling contaminant transport (e.g., Krishnaswami et al., 1982; Miller and Benson, 1983; Cederberg et al., 1985; Walsh et al., 1984; Yeh and Tripathi, 1991; Lichtner, 1988, 1992; Leslie, 1991).

Returning to the case where a linear isotherm is assumed to be valid, and $\Phi=0$, Eq. (2-27) can be adapted to $2 \mathrm{D}$ and $3 \mathrm{D}$ transport in the general form:

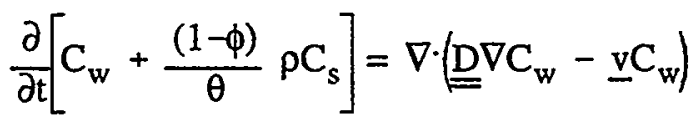

where

$$
\begin{array}{ll}
\underline{\underline{\mathrm{D}}} & =\text { hydrodynamic dispersion tensor, and } \\
\underline{\mathrm{v}} & =\text { mean pore velocity vector. }
\end{array}
$$

which can be modified in a fashion similar to the development outlined above in Eqs. (2-27) through (231). From Eq. (2-30), if $\theta$ does not vary with time, $R_{f}$ can be considered constant. For a homogeneous, incompressible medium under hydrologically-saturated conditions, $\theta=\phi$ and this may be a valid assumption. In a heterogeneous medium, however, volumetric moisture content varies as a complex function of both time and space, even under isothermal conditions, and $\theta$ cannot generally be considered constant. As a result, $R_{f}$ must also be evaluated as a function of time and space. Equation (2-32) also assumes that all available surfaces will be wetted, regardless of hydrologic saturation, and that there are no preferential zones of flow for mass transport through the volume (Clothier, 1984). Tritium experiments by Bond et al. (1982) suggested that a uniform migration front may be obtainable in partially-saturated column experiments. In practice, however, most geological media are heterogeneous, and preferential flow and transport through permeable layers and fractures all serve to complicate the application of a simplified model (Ababou, 1991).

In general, the convection-dispersion equation requires numerical solution, and simple analytical solutions are not readily derived. Van Genuchten and Alves (1982) provides an extensive compilation of ID analytical solutions to convection-dispersion. Two-dimensional analytical solutions are compiled in Cleary and Ungs (1978), while radial transport is considered by Moench and Ogata (1981). Applications of these solutions are discussed by Javandal et al., (1984). Bond and Phillips (1990) developed a 1D analytical solution for cation transport. The solution assumes a general non-linear adsorption isotherm, although the exact approach depends on the shape of the isotherm. The model has been applied to column experiments in the Ca-Na-K system using isotherms determined by batch experiments with fairly good agreement, except at short times.

As discussed above, advective transport is commonly assumed to be the principal mode of solute transport within permeable layers. Diffusion along chemical gradients is generally incorporated in the hydrodynamic dispersion coefficient. However, Travis and Nuttall (1987) suggested that, at the slow flow rates anticipated in the partially-saturated zone at Yucca Mountain $(<1 \mathrm{~cm} / \mathrm{yr})$, diffusion may become relatively more important as the magnitude of advective transport diminishes. Modeling the spreading of a nonreactive solute plume by mechanical dispersion during advective transport through a heterogeneous, 
layered medium requires complex velocity distributions. Gillham et al. (1984) proposed an advection/diffusion model that minimizes this complexity by invoking molecular diffusion along chemical gradients between layers of contrasting hydraulic conductivities. In this fashion, less permeable layers tend to act as temporary storage cells; solutes are concentrated at the expense of the more permeable layers, and contaminant transport is attenuated. This molecular interpretation requires knowledge of the contrast in unit hydraulic conductivities of the layers, layer thickness, and the molecular diffusion coefficient for the solute. Unfortunately, these parameters are not always known with any degree of certainty, and Gillham et al. (1984) were required to use empirical estimation techniques.

Fracturing of geologic media leads to heterogeneity at a variety of scales, greatly complicating the modeling of fluid flow and transport (Ababou, 1991). Deterministic modeling approaches have been modified for fracture flow in two basic ways. In the dual-porosity approach, the fracture network is treated as one effective porous medium, and the matrix as another. Effective values for hydraulic, transport, and sorptive properties are assigned to both fracture flow and matrix flow based on this assumption, and solute transport is described by a series of parallel equations similar to Eqs. (2-27) through (2-31). An additional term is required to consider cross-flow and transport between the fractures and the unfractured rock matrix (Dykhuizen, 1990). If coupling time scales are short relative to transport time scales, then fracture and matrix concentrations are comparable. Wilson and Dudley (1986) and Dykhuizen (1987) used this approach to describe 1D transport in the partially-saturated zone at Yucca Mountain. Neretnieks and Rasmuson (1984) also modified the approach to include radioactive decay during radionuclide transport in a fractured medium with varying velocity and block size.

The second deterministic approach to modeling fracture flow is to use pipe or parallel plane flow to model transport in individual fractures separately from the rock matrix. This strategy is only practical if fracture flow is controlled by a relatively small number of dominant fractures. For 2D transport, the dual porosity model requires a 2D grid for the entire system, and simultaneous solution of the entire set of nonlinear algebraic equations representing solute concentrations is necessary. In contrast, a discrete fracture model allows for 1D representation of fracture transport and requires sequential solution of subsets of the algebraic concentrations. In a test by Huyakorn et al. (1983), this led to a savings of $83 \%$ in CPU time. Huyakorn et al. (1983) modified the governing equations for discrete fracture flow to incorporate radionuclide decay and diffusion into the matrix. The authors also note, however, that extension into three dimensions may prove problematic.

\subsubsection{Nonequilibrium Solute Transport}

Reactive solute transport in the partially-saturated zone is usually described using the convectiondispersion equation appropriate to the dimensionality of the problem [Eq. (2-32)]. For example, Nair et al. (1990) modified the convection-dispersion equation to develop a 1D transport model for the three-phase (gas-liquid-solid) system in the partially-saturated zone. However, given the nonlinear nature of hysteretic effects, moisture content, hydraulic conductivity, and other hydraulic properties in the partially-saturated zone, there is considerable uncertainty in the applicability of this equation to transport under variablysaturated conditions (Valocchi, 1985). If the assumption of equilibrium is valid, and if the convectiondispersion equation is adequate to describe solute transport in a partially-saturated column experiment, it is expected that the eluted pulse of the solute will be roughly symmetrical in shape, increasing in concentration from zero to some maximum and decreasing back to zero. In practice, however, soils exhibit some structure, and elution curves from column experiments of both nonreactive and reactive solute transport are frequently asymmetrical (showing early breakthrough), and exhibit extensive tailing, 
asymptotically approaching some minimum, nonzero concentration. Van Genuchten and Wierenga (1976) also noted that tailing at larger pore volumes generally increases as porewater velocity decreases. Assuming that the convection-dispersion equation is appropriate, modeling kinetic solute transport in the partially-saturated zone has followed two basic approaches. The first approach involves dividing the flow regime into two physically-distinct domains. The second approach relies on chemical kinetics to reproduce observed profiles. Two reaction sites are proposed, one of which obeys chemical equilibrium, and the second of which follows nonequilibrium (usually first-order kinetic) processes.

\subsubsection{Two-Region Transport}

Two-region transport models are based on the existence of two physically distinct regions in a porous medium (Coats and Smith, 1964; van Genuchten and Wierenga, 1976). In one region, water is mobile and free to transport ions in solution. In the second region, water is considered immobile (or stagnant in some terminologies) in dead-end pore spaces. Mass transfer between the two regions is by diffusion alone. In one dimension, the two-region convection-dispersion transport equation becomes

$$
\begin{gathered}
\theta_{\mathrm{mo}} \frac{\partial \mathrm{C}_{\mathrm{w}, \mathrm{mo}}}{\partial \mathrm{t}}+f_{\mathrm{sm}} \rho_{\mathrm{b}} \frac{\partial \mathrm{C}_{\mathrm{s}, \mathrm{mo}}}{\partial \mathrm{t}}+\theta_{\mathrm{im}} \frac{\partial \mathrm{C}_{\mathrm{w}, \mathrm{im}}}{\partial \mathrm{t}}+\left(1-\mathrm{f}_{\mathrm{sm}}\right) \rho_{\mathrm{b}} \frac{\partial \mathrm{C}_{\mathrm{s}, \mathrm{im}}}{\partial \mathrm{t}}= \\
\theta_{\mathrm{mo}} \mathrm{D}_{\mathrm{mo}} \frac{\partial^{2} \mathrm{C}_{\mathrm{w}, \mathrm{mo}}}{\partial \mathrm{x}^{2}}-\theta_{\mathrm{mo}} \mathrm{v} \frac{\partial \mathrm{C}_{\mathrm{w}, \mathrm{mo}}}{\partial \mathrm{x}}
\end{gathered}
$$

where

$$
\begin{aligned}
& \text { mo, im = mobile and the immobile regions as subscripted, } \\
& \begin{array}{ll}
\mathrm{f}_{\mathrm{sm}} & \text { mass fraction of the solid phase that is in contact with the mobile fluid } \\
\theta, \rho_{\mathrm{b}} & \text { (dimensionless), and }
\end{array}
\end{aligned}
$$

Diffusion between the mobile and immobile regions is controlled by the relationship

$$
\theta_{\mathrm{im}} \frac{\partial \mathrm{C}_{\mathrm{w}, \mathrm{im}}}{\partial \mathrm{t}}+(1-\mathrm{f}) \rho_{\mathrm{b}} \frac{\partial \mathrm{C}_{\mathrm{s}, \mathrm{im}}}{\partial \mathrm{t}}=\alpha_{\mathrm{EMT}}\left(\mathrm{C}_{\mathrm{w}, \mathrm{mo}}-\mathrm{C}_{\mathrm{w}, \mathrm{im}}\right)
$$

where $\alpha_{E M T}$ is an empirical mass transfer coefficient $\left(\mathrm{s}^{-1}\right)$ between the stagnant and mobile waters. The degree of nonequilibrium represented in the system decreases as the rate of exchange between the two regions $\left(\alpha_{E M T}\right)$ increases relative to the rate of solute advection. It is important to note that local equilibrium is assumed to govern relationships in the mobile and immobile regions of the system. The two-region model, as defined by Eqs. (2-33) and (2-34), is therefore not a model limited by reaction kinetics, but rather uses concentration differences at points along the direction of flow to simulate disequilibrium.

The two-region approach has been used in a number of studies (van Eijkeren and Loch, 1984; de Smedt and Wierenga, 1984; Jinzhong, 1988; Mansell et al., 1988; Selim et al., 1976, 1990). Bidner and Vampa (1989) modified the approach to include the effects of equilibrium sorption and nonequilibrium adsorption/desorption. Immobile water has been inferred from partially-saturated column experiments that exhibit early breakthrough and asymmetric breakthrough curves (Bond and Wierenga, 1990). Skopp et al. (1981) noted that the fraction of stagnant water and the magnitude of the mass transfer coefficient are only defined by the results of experiments conducted under certain conditions; knowledge of the geometry and 
hydrologic properties of the medium is insufficient to predict the necessary parameters. De Smedt and Wierenga (1979) suggested that immobile water is indicated if fitting partially-saturated transport data requires the use of a dispersion coefficient much larger than that predicted for saturated fluid flow. De Smedt and Wierenga (1984) noted a roughly linear increase in $\alpha$ with increasing pore water velocity $\left(v_{m}\right)$, based on nonreactive transport column experiments. De Smedt and Wierenga (1984) also noted a positive linear correlation between mobile water $\left(\theta_{\mathrm{mo}}\right)$ and total water content $\left(\theta=\theta_{\mathrm{mo}}+\theta_{\mathrm{im}}\right)$ of the medium. Bond and Wierenga (1990) conducted nonreactive solute transport experiments under both steady and unsteady partially-saturated conditions to evaluate the two-region model. Under steady flow, early breakthrough and asymmetric breakthrough curves indicated the presence of small amounts of immobile water. Under unsteady flow conditions, however, the data were adequately described without invoking two regions. Bond and Wierenga (1990) attributed this to the differences in wetting patterns between the two cases. In the case of steady flow, water flow is already established and biased toward preferential flow paths, resulting in dead-end pore space. In contrast, wetting of the soil during the unsteady experiments carried the tracer into the wetted pore space by advection alone, with little or no contribution from diffusive mass transfer between mobile and immobile water. Mansell et al. (1990) incorporated a mobile/immobile two-region approach to nonequilibrium transport into the direct-coupled model of Valocchi et al. (1981a,b). Selectivity coefficients, a measure of the preference of the adsorbent for one aqueous species over another, are allowed to vary in space and time as a function of changes in solution normality and composition. The authors reported that the model was successful in predicting $\mathrm{Na}^{+}$and $\mathrm{Mg}^{2+}$ transport in laboratory experiments.

Skopp et al. (1981) further modified the two-region model to consider two homogeneous flow regions. One region consists of relatively rapid flow through macropores; the second region is governed by much slower matrix flow. Exchange between the two regions is modeled using a linear interaction coefficient. If the interaction coefficient is sufficiently large, the model reduces to the classical one-region convection dispersion equation [Eq. (2-32)]. In general, structured soils require small coefficient values, possibly due to flow channeling or clay dispersion. Reinterpreting the data of van Genuchten and Wierenga (1976) using this approach, Skopp et al. (1981) suggested that the mean diffusion path exceeds the average aggregate size, suggesting that the mobile/immobile assumption is inadequate.

\subsubsection{Two-Site Transport}

Many studies (Selim et al., 1976; Cameron and Klute, 1977; Jennings and Kirkner, 1984; Parker and Jardine, 1986; Kool et al., 1987) have proposed a two-site approach to modeling early breakthrough curves. One site is assumed to be governed by equilibrium sorption, and the second site assumes kinetic sorption reactions. As discussed by Nkedi-Kizza et al. (1984), adsorption on the equilibrium site (site 1) is governed by a linear sorption isotherm, while sorption at the nonequilibrium site (site 2 ) is governed by first-order, linear kinetics. The overall governing transport equation is

$$
\left[1+\frac{f_{s} \rho_{b} K_{d}}{\theta}\right] \frac{\partial C_{w}}{\partial t}+\frac{\rho_{b} \alpha_{2}}{\theta}\left[\left(1-f_{s}\right) \dot{K}_{d} C_{w}-C_{s, 2}\right]=D \frac{\partial^{2} C_{w}}{\partial x^{2}}-v \frac{\partial C_{w}}{\partial x}
$$

where

$$
\begin{array}{ll}
\mathrm{C}_{\mathrm{s}, 2} & =\text { concentration sorbed at site } 2 \text { ( } \mathrm{kg} \text { solute } / \mathrm{kg} \text { solid), } \\
\mathrm{f}_{\mathrm{s}} & =\text { fraction of sites that are governed by equilibrium sorption (dimensionless), } \\
\mathrm{K}_{\mathrm{d}} & =\text { linear sorption coefficient that is assumed valid for both sites }(1 / \mathrm{kg} \text { ), and }
\end{array}
$$


$\alpha_{2}=\mathrm{a}$ first-order kinetic rate coefficient for the nonequilibrium sorption sites $\left(\mathrm{s}^{-1}\right)$.

Other variables $\left(C_{W}, \rho_{b} D, v\right)$ are as defined previously.

Nkedi-Kizza et al. (1984) used a dimensionless parameterization of both the two-region and twosite models to demonstrate a mathematical equivalence of their dimensionless forms in terms of addressing nonequilibrium solute transport. Breakthrough curves (BTC) for ${ }^{45} \mathrm{Ca},{ }^{3} \mathrm{H}$, and ${ }^{36} \mathrm{Cl}$ transport in watersaturated column experiments were fit reasonably well by the models, including the tailing observed for the different BTCs. The authors noted, however, that because only the forms of the dimensionless equations are directly comparable, this equivalence is only valid for simulations of the net effect of retardation at a macroscale. Since dependent dimensionless variables (e.g., one dimensionless variable, $\mathrm{C}_{\mathrm{s}, 2}$ measures either solution concentrations in the immobile water for the two-region model, or the sorbed concentration at the kinetic site in the two-site model) differ in the two models, microscopic measurements (unspecified by the authors) are necessary for discrimination between the conceptual models. It is also important to note that the authors use a linear sorption isotherm to describe the equilibrium sorption/concentration relationships in the transport models.

\subsubsection{Stochastic Models}

Recent studies suggest inadequacies in the deterministic, convection-dispersion approach to simulating solute transport in the partially-saturated zone (e.g., Jury et al., 1983, 1986; Sposito et al., 1986; Roth et al., 1990a,b). Alemi et al. (1991) also indicated that retardation factors based on $\mathrm{K}_{\mathrm{d}}$ values from batch equilibrium experiments were inadequate to fit the results of selenium transport through partiallysaturated soil columns. As a result, recent efforts to model fracture and permeability-induced heterogeneities and partially-saturated transport have centered about the development of stochastic models. These models rely on performing large numbers of calculations to generate population statistics for randomly distributed hydraulic and transport properties. Statistical treatments of these probability distribution functions are then used to simulate heterogeneities at any scale. The specifics of the stochastic model techniques are beyond the intended scope of this text, and the reader is referred to other reviews (Dagan, 1986; van Genuchten and Jury, 1987; Ababou, 1991; Sagar, 1991) for a more comprehensive and detailed discussion of stochastic models.

Early studies of convection-dispersion using stochastic approaches have been largely limited to nonreactive solute transport (e.g., Bresler and Dagan, 1981; Dagan, 1988; Thompson and Gelhar, 1990). Most of the more recent studies that include reactive solute transport have generally modeled retardation using a linear $\mathrm{K}_{\mathrm{d}}$ approach (e.g., Jury et al., 1983; van der Zee and van Riemsdijk, 1987; Chrysikopoulos et al., 1990, 1992; Tompson and Knapp, 1989) for simple 1D, monocomponent systems with local equilibrium assumed. However, some studies have attempted to use more sophisticated sorption models to simulate chemical heterogeneity. Russo $(1989 \mathrm{a}, \mathrm{b})$ developed a ID stochastic model using a mixed-ion diffuse double layer to simulate solid/solution interaction for a simplified $\mathrm{Na}-\mathrm{Ca}-\mathrm{Cl}$ system. Comparison of predicted results to field data from an arid soil site at Bet Dagan, Israel, suggested that interactions between soil and solutions may affect transport under transient conditions, and increase the hydrologic heterogeneity of the medium. Cvetkovic and Shapiro (1990) used a first-order kinetic approach to account for retardation in their stochastic models, although at equilibrium, the model reduces to a linear $\mathrm{K}_{\mathrm{d}}$. Van der Zee (1990a) and van der Zee and Bolt (1991) attempted to address a maximum sorption limit using the Langmuir isotherm. Van der Zee and Boesten (1991) and Bosma and van der Zee (1993) used a random, normally distributed Fruendlich adsorption coefficient to model contaminant transport in a chemically heterogeneous ID system. The results calculated in this fashion differed significantly from 
those calculated using an analytical traveling wave solution (van der Zee, 1990b) and an average sorption coefficient. Tompson and Knapp (1991) and Tompson and Dougherty (1992a) have outlined methods for incorporating reaction progress models into stochastic approaches for a more realistic treatment of chemically reactive transport that uses mass balance/mass action considerations.

\subsubsection{Computer Codes}

Numerical codes that couple geochemical and transport models (hydrogeochemical models) have seen increasing use as tools for gaining insight into retardation processes and predicting the nature and extent of contaminant transport. Development of such comprehensive codes is being actively pursued by a number of research groups [LANL, LBL, Pacific Northwest Laboratory (PNL), SNL, ORNL, and EPRI] using a variety of approaches. An evaluation of existing codes should consider accuracy, efficiency, flexibility, and computational requirements, among other characteristics, in an attempt to find the best balance between model completeness and applicability.

Geochemical equilibrium can be represented by a set of nonlinear algebraic equations (Westall et al., 1976), while the efficiency of a given hydrogeochemical model is not only important for investigating the problem at hand, but it will also determine the ability of the program to be adapted to more complete (and complex) conceptual models. This efficiency, in turn, depends on both the approach taken toward coupling and the primary variables that are chosen to represent the problem of interest (Kirkner and Reeves, 1988; Reeves and Kirkner, 1988; Yeh and Tripathi, 1989). A number of approaches are available to couple geochemistry and transport models (e.g., Yeh and Tripathi, 1989; Lichtner, 1988; Tompson and Dougherty, 1992a). Direct coupling involves insertion of the nonlinear algebraic equations describing equilibrium geochemistry into the partial differential equations that describe transport. This results in a set of nonlinear partial differential equations that are solved simultaneously for geochemistry and transport, either explicitly or implicitly (Carnahan, 1987a). Another method, commonly called "twostep" coupling (Mangold and Tsang, 1991) poses the geochemistry and mass transport equations independently of one another, similar to the "operator splitting" method outlined in several recent studies (Bagtzoglou et al., 1992a; Tompson and Dougherty, 1992b; Valocchi and Malmstead, 1992). At each time step, the chemical components are transported by advection and dispersion into a representative elemental volume (REV). The solution is then equilibrated with the minerals present in the REV and the concentration of each primary variable is determined one by one using mass action and mass balance constraints. In this manner, the transport equations and the chemical relations are solved sequentially at each time step. Both direct substitution (Rubin and James, 1973; Valocchi et al., 1981a,b; Miller and Benson, 1983; Friedly and Rubin, 1992) and two-step coupling (Cederberg et al., 1985; Hostetler and Erikson, 1989; Yeh and Tripathi, 1991) have been used in hydrogeochemical models. In most cases, however, applications of these models have been limited to transport in the geologically short time frame of months to hundreds of years. One exception is the work of Garven and Freeze $(1984 \mathrm{a}, \mathrm{b})$ which used a two-step approach to couple output from the EQ3/6 Code (see Section 2.5.3.4) to a moving-particle, random-walk algorithm to simulate solute transport for tens of thousands of years.

As fluids out of chemical equilibrium with the host rock flow through porous media, dissolution and precipitation of minerals occurs, governed by the physical and chemical conditions of the system and the kinetics of the reactions involved. The reaction fronts formed in this manner can be quite sharp and propagate through the medium with time. This type of moving boundary problem is difficult to model using conventional numerical flow and transport models, requiring the use of small time steps to ensure convergence and accuracy. This limits the use of these traditional techniques to short time problems, as 
the number of time steps required to model geologic time scales on the order of thousands to millions of years is excessive. The sharpness of many of these reaction fronts (e.g., redox fronts) also requires fine spatial discretization in the vicinity of the front, further limiting the effectiveness of conventional methods.

As an alternative approach to the conventional finite difference and finite element approaches described above, a fourth approach has been adopted for the multicomponent reactive transport (Lichtner, 1985, 1988, 1992; Lichtner and Waber, 1992; Lichtner and Biino, 1992; Lichtner and Balashov, 1993). This approach involves the assumption of a quasi-stationary state approximation or multiple reaction path (MPATH) model. The stationary state consists of the chemistry of the fluid, and the reaction rates and mineral abundances in the host rock at a particular state of alteration as a function of distance from some inlet point. The fluid is considered as a single packet, reacting with minerals in the host rock as it moves through the medium. This results in a weakening of the coupling between minerals and fluid compositions in the model. Therefore, the fluid composition is calculated independently of changes in the volume fraction of minerals, significantly reducing the computational effort required. This allows longer time steps, which permits problems to be studied on a geological time scale. The key assumption that underlies the validity of this approach is that the time required to form a stationary state is rapid relative to the alteration of the host rock and the changes of temperature and composition of the inlet fluid. This assumption is supported because fluids are generally dilute in elements relative to the minerals in the host rock (Lichtner, 1988). In the case of pure advective transport, this effectively buffers the composition of the fluid at a stationary state; the fluid packet is open to minerals encountered by the packet, but is a closed system with respect to transfer of solutes within the fluid phase. A given stationary state exists until the reactions change the host rock sufficiently to require a new state to be formed. Considering MPATH in this manner allows modeling of complex geochemical systems. The mathematics describing the theoretical basis of the MPATH approach are reported in detail in Lichtner $(1985,1988)$.

Direct substitution and two-step coupling are used in most traditional finite difference/finite element approaches. For direct substitution, iteration is performed on the entire set of nonlinear partial differential equations at each time step; the coefficient matrix must be reformulated for each iteration, leading to extensive computer calculation time and large memory requirements (Cederberg, 1985). Alternatively, two-step coupling requires the solution of transport and chemical equations in sequence. This eliminates the need to reformulate the coefficient matrices within each time step, using computer resources more effectively (Cederberg, 1985). Cederberg (1985) and Yeh and Tripathi (1989) indicated that a two-step coupling approach is more than 30 percent faster than direct coupling for a given problem. Yeh and Tripathi (1989) also reported that a two-step approach is the most efficient choice among the traditional techniques for larger 2D and 3D problems, given likely limits on computational speed and memory storage. Numerical techniques employed in matrix formulation and solution, and in iteration, will also have an effect on run time and therefore, run costs (Barry, 1990). Several studies (Yeh, 1985; Reeves and Kirkner, 1988; Yeh and Tripathi, 1989; Siegel et al., 1989) have evaluated a variety of iterative strategies (e.g., Picard, Newton-Raphson, successive overrelaxation, Gauss-Seidel) and identified convergence problems. Because of this, time steps are generally short relative to geologic time scales, on the order of hours to days. Even on a supercomputer, CPU times are large and run-time costs can amount to many thousands of dollars for a single 10,000 year simulation on a scale similar to Yucca Mountain (Siegel et al., 1989). The quasi-stationary approximation is not limited to small time steps for convergence [e.g., Lichtner (1988) uses a time step of up to 200 years], and is better suited to problems on a geologic time scale (Lichtner, 1988; 1992).

Because a reactive transport code is envisioned as a part of an interactive approach to performance assessment, codes requiring extensive supercomputer time are at a distinct disadvantage as 
geochemical codes, the newly developed geochemical module, EQMOD, enables HYDROGEOCHEM to consider equilibrium aqueous complexation, ion exchange, surface complexation, precipitation/dissolution, redox, and acid-base reactions. No database is provided with the code and all relevant thermodynamic data must be entered in the input file. The code has been applied to several laboratory and field systems during development and testing, including radionuclide transport and leaching of uranium mill tailings (Yeh and Tripathi, 1991).

\subsubsection{DYNAMIX}

The DYNAMIX code is based on the transport code TRUMP and the geochemical equilibrium code PHREEQE (Liu and Narasimhan, 1989a,b). Developed at the University of California, Berkeley, DYNAMIX is a 3D code that employs a two-step coupling approach to model reactive solute transport due to advection, dispersion and diffusion. The code is designed specifically to handle aqueous complexation, redox processes, ion exchange, and precipitation/dissolution. The code is also being modified to use partial equilibrium to consider surface reaction-limited kinetic mineral dissolution (Liu and Narasimhan, 1989a,b). The code has been tested against four other reactive transport codes, PHASEQL/FLOW (Waish et al., 1982), THCC (Carnahan, 1987b,c), PHREEQM (Glynn et al., 1991) and FASTCHEM (Narasimhan et al., 1992) and found to be in reasonable agreement except for transport of redox sensitive elements such as $\mathrm{Cu}, \mathrm{Mn}, \mathrm{As}$, and $\mathrm{Cr}$. DYNAMIX has been applied to several hypothetical transport problems including uranium transport, supergene copper enrichment, and reactive transport of As and Se (Narasimhan et al., 1992; Liu and Narasimhan, 1989b). Numerical convergence is ensured by fine scale discretization to keep cell Peclet numbers small and by choosing small time steps. This rapidly leads to increased computation time, limiting application with regard to geologic scales in time and space. For example, a $25 \mathrm{~m} \times 1000 \mathrm{~m} 2 \mathrm{D}$ simulation of leaching and transport of As and Se from fly ash over 30 yrs, DYNAMIX required approximately 3.4 CPU hrs of CRAY supercomputer time (Liu and Narasimhan, 1989b).

\subsubsection{CHEQMATE}

Developed at Harwell Laboratory in the United Kingdom, the finite difference CHEQMATE (CHemical EQuilibrium with MigrAtion and Transport Equations) code originally considered only molecular diffusion along concentration gradients and electromigration (Haworth et al., 1988a). The code has since been extended to consider advective transport of species through a series of heterogeneous media under constant-flow conditions (Haworth et al., 1988b) and spherical geometries. The code is able to model 1D transport using iterative two-step coupling and an assumption of local equilibrium. The chemical equilibrium is based on the PHREEQE geochemical code and relies on the PHREEQE thermodynamic database. Since the transport equations are only derived in terms of concentrations, and thermodynamic activities are not considered, the code is limited to dilute aqueous solutions. Aqueous complexation, precipitation/dissolution, redox, and acid-base reactions are modeled, but adsorption/desorption, ion exchange, and reaction kinetics are not. The code is written in FORTRAN 77 and has been adapted to IBM mainframe and CRAY2 computer systems. By scaling down the thermodynamic database to 10 elements, 50 species, and 20 minerals, a model has been adapted to IBM PC use (Haworth et al., 1988a).

\subsubsection{TRANQL}

The TRANQL code, developed at Stanford University, has been used to model multicomponent transport of $\mathrm{Cd}, \mathrm{Co}, \mathrm{Br}$, and $\mathrm{Cl}$ (Cederberg, 1985; Cederberg et al., 1985). The code is based on the transport code ISOQUAD (Pinder and Gray, 1977) and MICROQL (Westall, 1979), a scaled-down version 
an elaborate, computationally slow code will become the limiting step in performance assessment calculations. Current investigations into massively parallel computing hold promise for overcoming computational limitations (e.g., Barry, 1990; Sagar et al., 1992; Bagtzoglou et al., 1992b).

Thermodynamic data are of critical importance in modeling any geochemical system. A given hydrogeochemical code should use an established, current database, that has been tested for accuracy and internal consistency, and is broad enough to include a relatively complete set of species, minerals, and complexes that are likely to be encountered in the geologic environment of interest (Kincaid et al., 1984a,b; Krupka et al., 1988). In addition, the necessary data for modeling important processes (sorption, precipitation/dissolution, ion exchange, activity, etc.) should be available. The database should be able to incorporate both updated information and additional data, as needed for a given system. A readilyavailable, public-domain database will have additional advantages of being tested through application to a wide variety of problems applications, and will also benefit from a general interest on the part of the research community in keeping the database consistent, accurate, and current. Finally, a relatively-complete database should be readily accessible to the code in order to adapt the model to a variety of systems.

Although the degree of "user-friendliness" must not be developed at the expense of the accuracy or efficiency of a program, it should be considered in code selection (Siegel et al., 1989). The program should be accessible to a variety of users with varying levels of computer skills. Complicated or awkward formatting of input can make application of the model difficult, and limit its usefulness as a tool to study a wide variety of systems or perform sensitivity analyses. Rigid input can also inhibit modification or correction of the input, perhaps leading to false starts and inaccurate data entry. Because these codes deal with potentially large numbers of chemical species and long time intervals and distances, post-processing of the data is also important as an aid in interpreting the output from the hydrogeochemical model.

This section is not intended to be an exhaustive analysis of existing reactive transport codes, but is intended to illustrate the kinds of hydrogeochemical codes available. Information about the code characteristics and the techniques was largely collected from users' manuals and key references; additional information is available in the users' manuals and in Morrey et al. (1986), Siegel et al. (1989) and Mangold and Tsang (1991). In many instances, unnamed hydrogeochemical codes have been developed for a particular problem (Rubin and James, 1973; Valocchi et al., 1981a,b; Walsh et al., 1982), and are not generally considered in this discussion. Three codes considered (NEFTRAN II, TRACR3D, and PORFLOW) are examples of solute transport codes that do not explicitly account for the geochemistry of the water/rock system. The remainder of the codes are hydrogeochemical models. All codes are written in FORTRAN. With the exception of DYNAMIX, CHMTRNS, and MPATH, all of the codes are isothermal, equilibrium models, and do not currently incorporate reaction kinetics. It is also important to note that hydrogeochemical modeling is a dynamic area of research, and future developments may result in new, more powerful codes or in extensive modification to the existing models.

\subsubsection{HYDROGEOCHEM}

Developed for supercomputing facilities at Oak Ridge National Laboratory (ORNL), the 2D finite-element code HYDROGEOCHEM is able to model steady-state or transient flow conditions while considering a variety of chemical processes during reactive solute transport (Yeh and Tripathi, 1990, 1991). A two-step sequential approach is generally used to couple equilibrium chemistry and transport, although a one-step coupling is available for comparison of run-time requirements. The transport module of HYDROGEOCHEM is based on the earlier fluid flow code FEMWATER (Yeh, 1987) and the transport code FEMWASTE (Yeh and Ward, 1981). Based on the approach used in the EQ3/6 and PHREEQE 
of the MINEQL code. TRANQL has been evaluated by Siegel et al. (1989). The model is currently designed for 1D simulation employing a mainframe computer system. The current system is able to model ion exchange and surface complexation sorption processes, but cannot handle precipitation/dissolution reactions. Pre- and post-processing routines have been developed to facilitate data entry and interpretation. The code is currently limited to a maximum of two mobile components and one sorbing substrate. Siegel et al. (1989) concluded that code flexibility is limited by its database, and for a simple system (14 complexes), reactive solute transport for a distance of $10 \mathrm{~km}$ over 10,000 yrs would require about $40 \mathrm{hrs}$ of CPU time on the SNL CRAY-1.

\subsubsection{FASTCHEM}

The FASTCHEM system (Hostetler and Erikson, 1989; Hostetler et al., 1989) was developed by PNL for EPRI, and is based on the SATURN transport code and the MINTEQ geochemical code (Morrey et al., 1986). The program is modular in design, and incorporates a networked PC workstation for constructing input files to an online mainframe computer for transport and geochemical calculations. Post-processing of the output is performed on the mainframe for subsequent downloading and graphic display at the workstation. The code uses a modified version of the MINTEQ database and is valid for the temperature range 25 to $100^{\circ} \mathrm{C}$ (Krupka et al., 1988; Criscenti et al., 1989). A Markov hydrological model is used to simulate solute movement through advection, diffusion, and hydrodynamic dispersion (Kincaid, 1988). This method involves discretizing a streamtube into a number of arbitrarily shaped bins. The total concentration of each solute in each bin is then expressed as an entry in a state vector, and the Markov transition matrix is used to predict the evolution of the state vector through time. Two-step coupling of geochemistry and transport is used for efficient computation. Only longitudinal dispersion is modeled and molecular diffusion is neglected; a series of parallel, 1D, noninteracting streamtubes is used to model 2D (and in theory 3D) systems. Because of the streamtube construction, FASTCHEM is somewhat limited in its ability to model transient conditions. The 2D flow code EFLOW is run until steady-state is achieved. The steady-state flow field generated in this fashion is then processed into the necessary streamtubes by the code ETUBE. In order to simulate transient changes in hydraulic properties of the medium, it is necessary to redefine the boundary conditions at the appropriate timestep, and then run the EFLOW code again to achieve a steady-state flow field. ETUBE is executed again, and a new set of streamtubes is defined. The module ECHEM calculates equilibrium geochemical processes modeled in FASTCHEM including speciation, complexation, oxidation/reduction, and several different adsorption models $\left(\mathrm{K}_{\mathrm{d}}\right.$, empirical isotherms, ion exchange, surface complexation). Activity relationships are modeled using the Davies or the extended Debye-Hückel equations. The module EICM couples the chemical input from ECHEM to the flow system. Because EICM does not explicitly simulate oxidation/reduction, redox reactions are not evaluated in the coupled transport problem (Narasimhan et al., 1992). Instead an arbitrary $\mathrm{pE}$ (electron potential, see Appendix D) is calculated based on a user selected $\mathrm{Fe}^{+2} / \mathrm{Fe}^{+3}$ couple. This is likely to be a severe limitation for modeling transport of redox sensitive elements such as actinides (Liu and Narasimhan, 1989b; Lichtner, 1992, Lichtner and Waber, 1992; Narasimhan et al., 1992). FASTCHEM has been compared to a 1D analytical solution for reactive transport (Ungs et al., 1986) with reasonable success (Hostettler and Erikson, 1989). The program has also been compared to the DYNAMIX code (Lin and Narasimhan, 1989a,b) with reasonable agreement except for transport involving redox sensitive species (Narasimhan et al., 1992). FASTCHEM has also been applied to hypothetical transport problems involving $\mathrm{Cu}, \mathrm{Cd}, \mathrm{Mn}, \mathrm{Zn}$, and to heavy metal transport from two fly-ash disposal sites in Illinois (Narasimhan et al., 1992). 


\subsubsection{CTM}

The CTM (Chemical Transport Model) code (Morrey and Hostetler, 1985; Erikson et al., 1990) is currently in development at PNL for the Low-Level Waste Management Division of the NRC, and incorporates many of the same methods and approaches as the proprietary FASTCHEM code. The database is the same as the FASTCHEM code, with further modifications to include the compilations of Wagman et al. (1982) and the uranium data of Tripathi (1984). The current version of the code has only been set up for $1 D$, isothermal simulations assuming equilibrium. While the CTM code is currently designed to run on an IBM PS/2 Model 70, future developments for modeling 2D and 3D systems will require mainframe capabilities. Pre- and post-processing are available for data input and output through an interactive system. Output is designed to take advantage of several commercial graphics software packages currently available for the PS/2 system (SURFER/GRAPHER).

\subsubsection{CHEMTRN}

The CHEMTRN code was developed at LBL, and has been applied to nuclear waste isolation and contaminant transport (Miller, 1983; Miller and Benson, 1983). The code uses the one-step directcoupling method, and is therefore more cumbersome than the two-step codes discussed above. CHEMTRN is an equilibrium code, and is similar to FASTCHEM/CTM in that it employs a 1D streamtube to model fluid flow. Due to the one-step coupling, however, storage limitations will become a problem in extending the model to multiple dimensions. The code is able to model ion exchange, surface complexation (triplelayer model only), and precipitation/dissolution reactions as retardation mechanisms. No $\mathrm{K}_{\mathrm{d}}$ or sorption isotherms are available for the code as it is currently configured. The model is limited to saturated flow through a homogeneous porous medium at constant temperature. In applications to date, the model has not employed an extensive, established thermodynamic database. The user is required to provide all of the reaction stoichiometries, equilibrium coefficients, CEC, sorption parameters, and other variables for each run using a formatted batch input. It does appear that data can be included from a variety of sources, but a database would have to be developed and modified for extensive use of this code.

\subsubsection{CHMTRNS}

The CHMTRNS code was also developed at LBL (Noorishad et al., 1987), and represents a version of CHEMTRN that has been modificd to include reaction kinetics, carbon isotope fractionation, and nonisothermal behavior. Multiple sorption models can be used in a given run. Formatted batch input is used for defining the problem and inputting the initial and boundary conditions. Each input deck consists of a minimum of twenty "cards," each card consisting of from one to seven parameters. Although the elaborate input makes the code flexible enough for application to a variety of systems and situations, many of the parameters must be defined by the user, leading to complex data input. In addition, much of the thermodynamic, kinetic, and sorption data must be entered each time the model is run, limiting the ability of the code to perform multiple runs for sensitivity analysis. No pre- or post-processing of the data is currently available to help in data entry or interpretation. Data entry is similar to that for CHEMTRN.

\subsubsection{MPATH}

MPATH uses the quasi-stationary state approximation and is described in detail in Lichtner (1992). As currently implemented in MPATH, a Lagrangian reference frame is used to consider 1D pure advective flow and transport at a constant velocity in a saturated homogeneous porous medium. Either flow through an open system or batch reaction in a closed system can be modeled. In the open system, minerals are not permitted to back-react with the fluid. Constant porosity and permeability are currently 
assumed, although the approach is not limited to this assumption (Lichtner, 1992). An adaptive grid algorithm is used to adjust the time step to more finely discretize the flow path in time and space at fronts involving sharp changes in concentration, such as reduction-oxidation or hydrolytic' reaction fronts. A stationary state is generally assumed to persist until a mineral dissolves completely or until surface area, permeability, or porosity change significantly. In moving to each new stationary state, MPATH verifies global mass balance for the system (Lichtner, 1992). Geochemical data for minerals and aqueous species are provided by either the EQ3/6 database or SUPCRT92 generated equilibrium constants (Johnson et al., 1991). Two types of reactions are considered; homogeneous reactions between species in the aqueous phase, and irreversible heterogeneous reactions between minerals and the fluid. Local equilibrium is considered as the limiting case of a kinetic description where the reaction rate constant approaches infinity. MPATH can therefore be used to simulate local equilibrium by choosing a rate constant that is sufficiently large (Lichtner, 1992). The user can consider either isothermal systems or specify temperature profiles as a function of time and space, with the stipulation that the time scale of changes to the profile are slow with respect to the time necessary for the fluid composition to achieve a stationary state. Ionic strength effects on activity coefficients are calculated based on the extended Debye-Hückel formulation [Eq. (218)]. To simulate kinetic barriers to precipitation, minerals can be allowed to remain supersaturated until some threshold value is reached. Rather than using an operational electron, such as HYDROGEOCHEM (Yeh and Tripathi, 1991) does, MPATH considers reduction-oxidation in terms of actual species in solution. This ensures balance of electrons in the solution (Lichtner, 1992).

MPATH has been used to model several natural systems including: (i) Uranium transport during weathering at the Osamu Utsumi uranium mine, Poços de Caldas, Brazil for time spans up to two million years (Lichtner and Waber, 1992), and (ii) supergene enrichment of copper protore in the Cu-Fe-S system (Lichtner and Biino, 1992). The results are in good qualitative agreement with field observations. Test applications of MPATH have also included migration of uranium and selenium in response to redox conditions, and weathering and hydrothermal alteration of alaskite (Lichtner, 1992). One important result of these applications is that given enough time, the kinetic model results (i.e., solute concentration, reaction front velocities, and mineral abundance) approach those of the local equilibrium model, exhibiting a relative insensitivity to the rate law or rate constants chosen (Lichtner and Waber, 1992; Lichtner and Biino, 1992; Lichtner, 1988, 1992).

\subsubsection{NEFTRAN, TRACR3D, and PORFLOW}

NEFTRAN II (Olague et al., 1991), TRACR3D (Travis, 1984), and PORFLOW (Runchal and Sagar, 1992) are solute transport codes that use simple representations of sorption such as linear isotherms $\left(\mathrm{K}_{\mathrm{d}}\right)$, and do not explicitly consider the geochemical equilibria involved in contaminant migration. As such, they are not suitable for examining specific geochemical processes. However, because the bulk of the calculation time involved in modeling reactive solute transport is spent calculating the geochemistry, solute transport codes are much quicker. They can therefore be adapted to model 3D transport, radionuclide decay, and fractured media transport without overextending computer resources. In addition, they can be more finely discretized, and the physical aspect of solute transport can be examined in more detail.

\subsubsection{Summary and Conclusions}

Coupling of geochemistry and transport have been accomplished in several ways (Cederberg et al., 1985; Carnahan, 1987a; Yeh and Tripathi, 1989; Lichtner, 1988). Direct coupling results in a single set of nonlinear partial differential equations which are solved simultaneously for species distribution; two- 
step coupling poses the nonlinear algebraic expressions for geochemistry separately from the partial differential equations for mass transport and solves them sequentially at each time step. These conventional approaches can be solved numerically using either finite element or finite difference techniques. A two-step coupling approach tends to be more computationally efficient than direct coupling (Cederberg, 1985; Siegel et al., 1989). However, these methods generally require a small time step to ensure convergence, and they have not been applied to time scales on the order of thousands to millions of years. As an alternative to these approaches, a quasi-stationary state approximation has been used to model kinetically controlled mineral precipitation/dissolution. Larger time steps can be used in this approach, and field scale problems have been simulated over geologic time scales. An additional consideration is the use of stochastic models in probabilistic assessment of the role of partially-saturated flow and geologic heterogeneities in solute transport. These models frequently rely on performing large numbers of calculations (e.g., Monte Carlo approach) to develop the necessary population statistics. Computer intensive geochemistry calculations may be inappropriate for this type of approach and severely limit the application of currently available hydrogeochemical codes in stochastic modeling. Research into the application of parallel computing technologies to reactive solute transport may offer a way to overcome some of these computational limitations.

The $\mathrm{H} \Rightarrow \mathrm{C}$ coupling is considered of critical importance to predicting release and transport of radionuclides. The importance of hydrologic conditions on corrosion processes is widely discussed in the literature, where it is noted that the corrosion rates of metallic waste containers is considerably lower in dry environments than in aqueous (wet) environments. Similarly, the rates of waste form dissolution and mobilization are considerably increased in aqueous environments (Shreir, 1970). The chief method of radionuclide transport is anticipated to be as dissolved species in the liquid phase or, in the case of ${ }^{14} \mathrm{C}$ and possibly ${ }^{129} \mathrm{I}$, in the gas phase (DOE, 1988). For transport in either the liquid or gas phase, the importance of the $\mathrm{H} \Rightarrow \mathrm{C}$ coupling is well documented in the literature and considered to be important.

\subsection{CHEMICAL $\Rightarrow$ HYDROLOGIC}

Chemical processes are expected to directly affect hydrologic processes through either (i) changes in the mineral deposition on fracture surfaces which may affect liquid imbibition into the rock matrix, (ii) fracture and pore healing/plugging, (iii) chemical osmosis (which is also discussed in Section 3.2.2), or (iv) changes in fluid properties (e.g., vapor pressure, viscosity). Each is discussed further.

\subsubsection{Mineral Deposition on Fracture Surfaces}

Gallegos et al. (1992) have studied the influence of fracture coatings on the transfer of water between the fracture and the matrix in a partially-saturated medium. It was determined that the mineralogy of the fracture surfaces may be important in restricting the imbibition of water into the matrix. This is considered theoretically important, especially for the validity of the equivalent continuum model (ECM) which is discussed in Appendix B of this report. The ECM assumes hydrologic equilibrium between the fractures and the matrix. If a fracture coating exists, then it may sufficiently reduce the rate of imbibition to cause the fracture and matrix to be out of hydrologic equilibrium. It was found (Gallegos et al., 1992) that the effect of the mineralized face ranged from negligible to strongly restricting water uptake into the matrix. The results are considered preliminary and future laboratory experiments are planned (Gallegos et al., 1992). The chemical coating on fracture surfaces is closely related to the dissolution, movement, and precipitation of minerals associated with fracture healing, which is discussed next. 


\subsubsection{Fracture Healing}

Lin and Daily (1989) and Daily et al. (1987) conducted laboratory experiments to determine the hydrologic transport properties of Topopah Spring tuff. One conclusion of that work is that the hydrologic permeability of intact rock is essentially independent of drying/wetting cycles at elevated temperatures for prolonged periods of time. Fractured rock, however, exhibited permeability decreases of more than 3 orders of magnitude during a 6-month experiment (Daily et al., 1987). The decrease of permeability was attributed to "fracture healing" which consists of dissolution and precipitation of minerals such as silica. Hence, the permeability of fractured tuff was found to be strongly dependent on temperature and time.

The implication of fracture healing on the near field hydrology has been studied by Lin and Daily (1989). There, it was hypothesized that a heat-generating waste package will sufficiently elevate the near-field temperatures and induce fracture healing so that an envelope of low-permeability rock will surround the waste packages (Lin and Daily, 1989). The envelope of low-permeability rock would diminish possible infiltration of water into the vicinity of the waste packages, hence help keep the packages dry for extended periods of time. Numerical simulations of Travis and Nuttall (1987) predicted similar results modeling 2D transport in Yucca Mountain.

\subsubsection{Chemical Osmosis}

Chemical osmosis describes a condition where a gradient of solute concentration causes a flux of solvent (i.e., liquid fluid). The process of osmosis is frequently discussed in textbooks in the context of "reverse osmosis" which is applied to desalinization and ultrafiltration of water. In textbook discussions, osmosis is described as occurring through a semipermeable membrane which preferentially passes water molecules but does not permit the passage of solutes. In the broader sense, osmosis has been applied to diffusional processes through a porous medium which provide a driving potential for the flux of water.

Carnahan (1984a) describes thermodynamically-coupled mass transport processes in a saturated clay, in which chemical osmosis is described. Here chemical osmosis describes the flux of solvent driven by a gradient in solute concentration

$$
\vec{v}_{L}-\nabla C_{I}
$$

where

$$
\begin{array}{ll}
\overrightarrow{\mathrm{v}}_{\mathrm{L}} & =\text { solvent (liquid) velocity }(\mathrm{m} / \mathrm{s}), \\
\nabla & =\text { gradient operator }\left(\mathrm{m}^{-1}\right), \text { and } \\
\mathrm{C}_{\mathrm{I}} & =\text { concentration of species } \mathrm{I} \text { (solute) in the liquid (moles } / \mathrm{kg} \mathrm{H}_{2} \mathrm{O} \text { ). }
\end{array}
$$

Carnahan (1984b) notes that there is a lack of experimental data on chemical osmosis and other thermodynamically-coupled constitutive properties (e.g., thermal osmosis, the Soret effect). By estimating coefficients from the literature, Carnahan (1984b) conducted numerical simulations. The simulations indicated that chemical osmosis can cause a significant flux of water, up to 3 or 4 orders of magnitude greater than Darcy's flux in a saturated clay. 
Jamet et al. (1990) expanded on the concept of constitutively-coupled processes (which is also discussed in Section 3.2.2) based on the ideas from nonequilibrium thermodynamics. Again, chemical osmosis was considered with a host of other couplings. One conclusion is that, under very idealized situations, the secondary couplings (e.g., chemical osmosis) may be more important than the primary couplings (Darcy's law, Fick's law, Fourier's law). The relative importance of the couplings depends on the values of the phenomenological coefficients, which unfortunately are typically not available in the literature.

\subsubsection{Fluid Properties}

Chemical processes are expected to influence fluid properties such as density, viscosity and vapor pressure. It is established that the density of water is affected by the introduction of dissolved species into solution (e.g., Sourirajan and Kennedy, 1962; Potter and Brown, 1977; Bischoff, 1991). In general, the density of saline water (liquid) is greater at a given temperature and pressure than that of pure water (Figure 2-25). In hydrothermal environments, hydrohaline convection has been proposed where salinity dependent density gradients add to thermally driven convection and enhance and focus fluid flow and heat transfer (Forster and Smith, 1990).

The presence of dissolved gases such as $\mathrm{CO}_{2}$ can also change fluid density (Takenouchi and Kennedy, 1965; Bowers and Helgeson, 1983; Sterner and Bodnar, 1991). At low pressures and temperatures, however, the solubility of $\mathrm{CO}_{2}$ in pure water is small. As a result of this low solubility, the fluid will separate into a low-density, high $\mathrm{CO}_{2}$ phase (vapor) and a second high-density, $\mathrm{H}_{2} \mathrm{O}$ rich liquid phase. At temperatures and pressures on the order of those anticipated at Yucca Mountain, $\mathrm{CO}_{2}$ is almost completely insoluble in pure $\mathrm{H}_{2} \mathrm{O}$, and the effect of dissolved $\mathrm{CO}_{2}$ on water density is negligible. The addition of $\mathrm{NaCl}$ to the system further decreases the $\mathrm{CO}_{2}$ solubility (Takenouchi and Kennedy, 1965; Bowers and Helgeson, 1983).

As shown in Figure 2-26, fluid viscosity also increases with increasing salinity at constant temperature and pressure (Forster and Smith, 1990). Increasing viscosity tends to reduce advective hydrologic transport.

Under certain conditions, the direct effects of salinity on water density and viscosity may be overshadowed by the indirect effects of shifting the liquid-vapor curve and the critical point to higher pressures and temperatures with increased salinity (Figure 2-27) (Bischoff, 1991). The shift in the critical point with salinity can be quite pronounced, from $374{ }^{\circ} \mathrm{C}$ and 220 bars for pure water to $500{ }^{\circ} \mathrm{C}$ and 582 bars for $13.6 \mathrm{wt}$ percent $\mathrm{NaCl}$. This shifts the position of the near-critical region to a different pressuretemperature region. Dissolved $\mathrm{CO}_{2}$ also shifts the critical point of water to higher pressures and lower temperatures (Figure 2-28). At low temperatures and pressures, however, the effect of $\mathrm{CO}_{2}$ is negligible, except at very high mole-percent $\mathrm{CO}_{2}$, and the shift in the P-T trajectory of the liquid-vapor curve is not appreciable. Assuming behavior analogous to the pure water system, fluid properties in the near-critical region such as density, heat capacity, and enthalpy can vary widely over narrow ranges in temperature and pressure (e.g., Johnson and Norton, 1991).

Like liquids, the physiochemical properties of gases are controlled at a basic level through varying gas composition as a function of pressure and temperature. Largely controlled by contact with the atmosphere, water vapor, carbon dioxide, and oxygen are the most critical gases in most hydrologically partially-saturated natural systems. The presence of buffers in groundwaters, such as a $\mathrm{Fe}^{2+} / \mathrm{Fe}^{3+}$ redox 


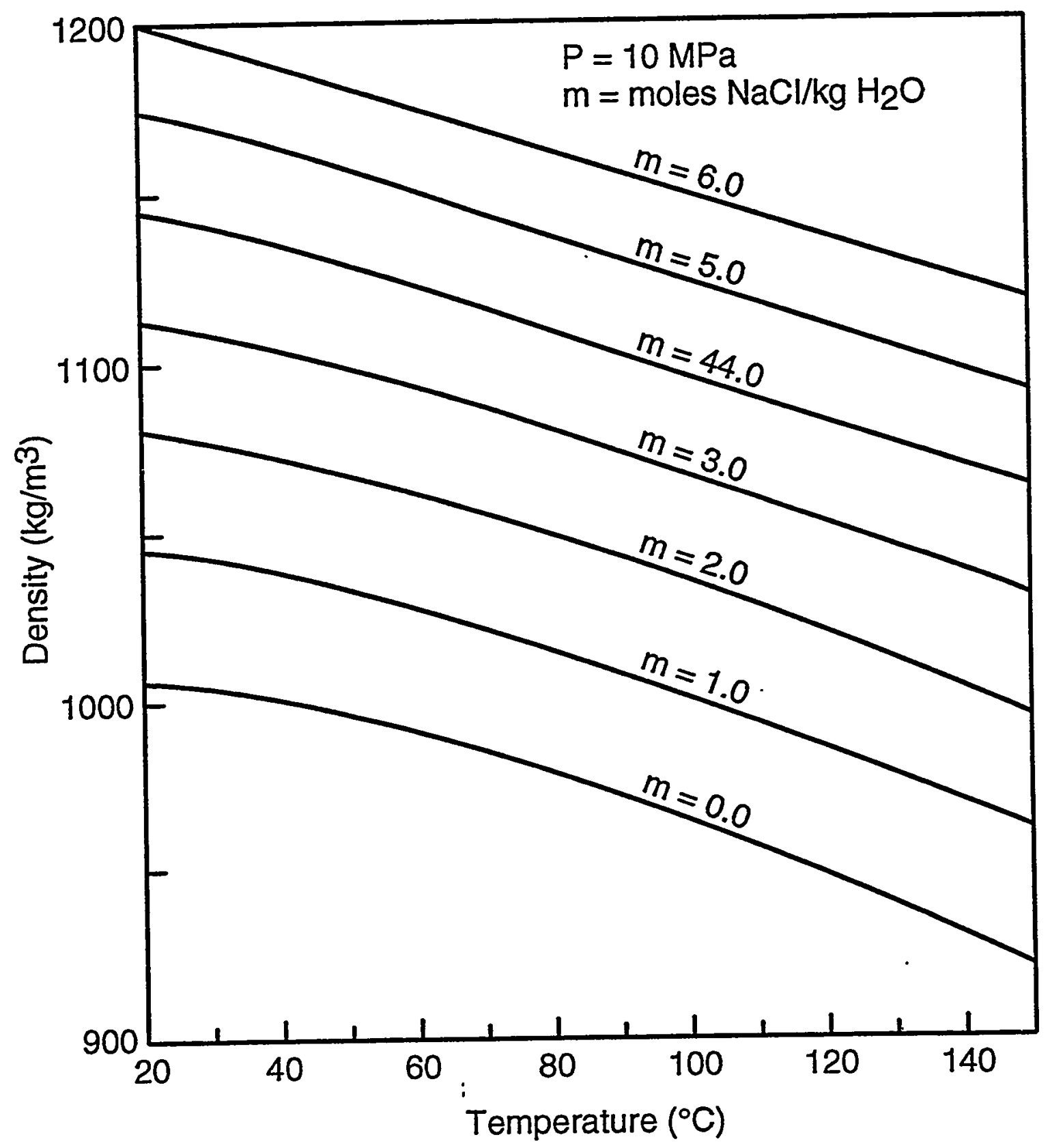

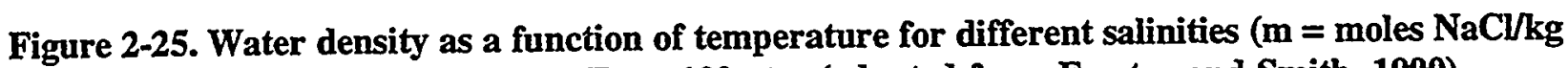
$\mathrm{H}_{2} \mathrm{O}$ ) at a constant pressure of $10 \mathrm{MPa} \approx 100 \mathrm{~atm}$ (adapted from Forster and Smith, 1990). 


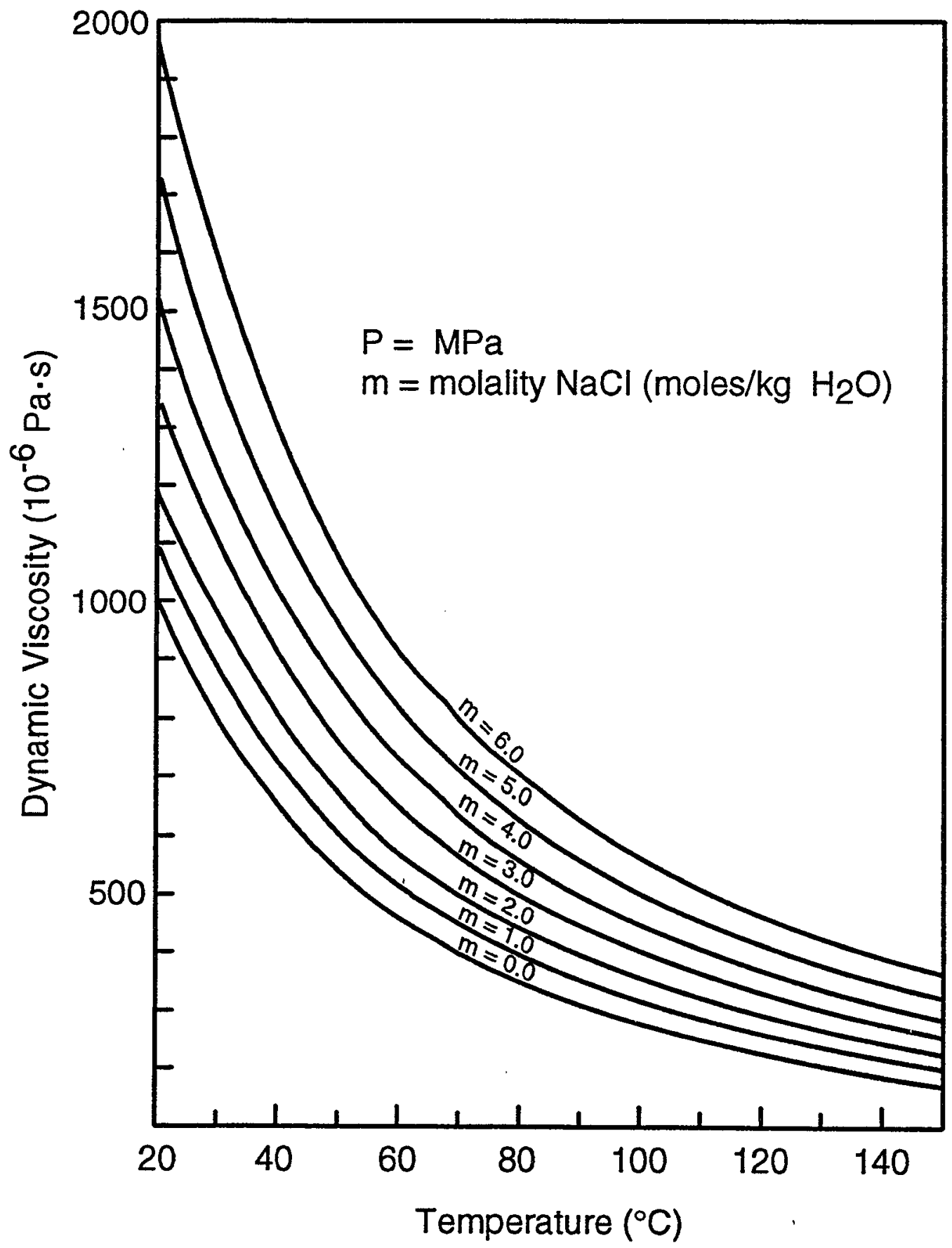

Figure 2-26. Dynamic viscosity of water as a function of salinity for constant $P=10 \mathrm{MPa}=100 \mathrm{~atm}$ (adapted from Forster and Smith, 1990). 


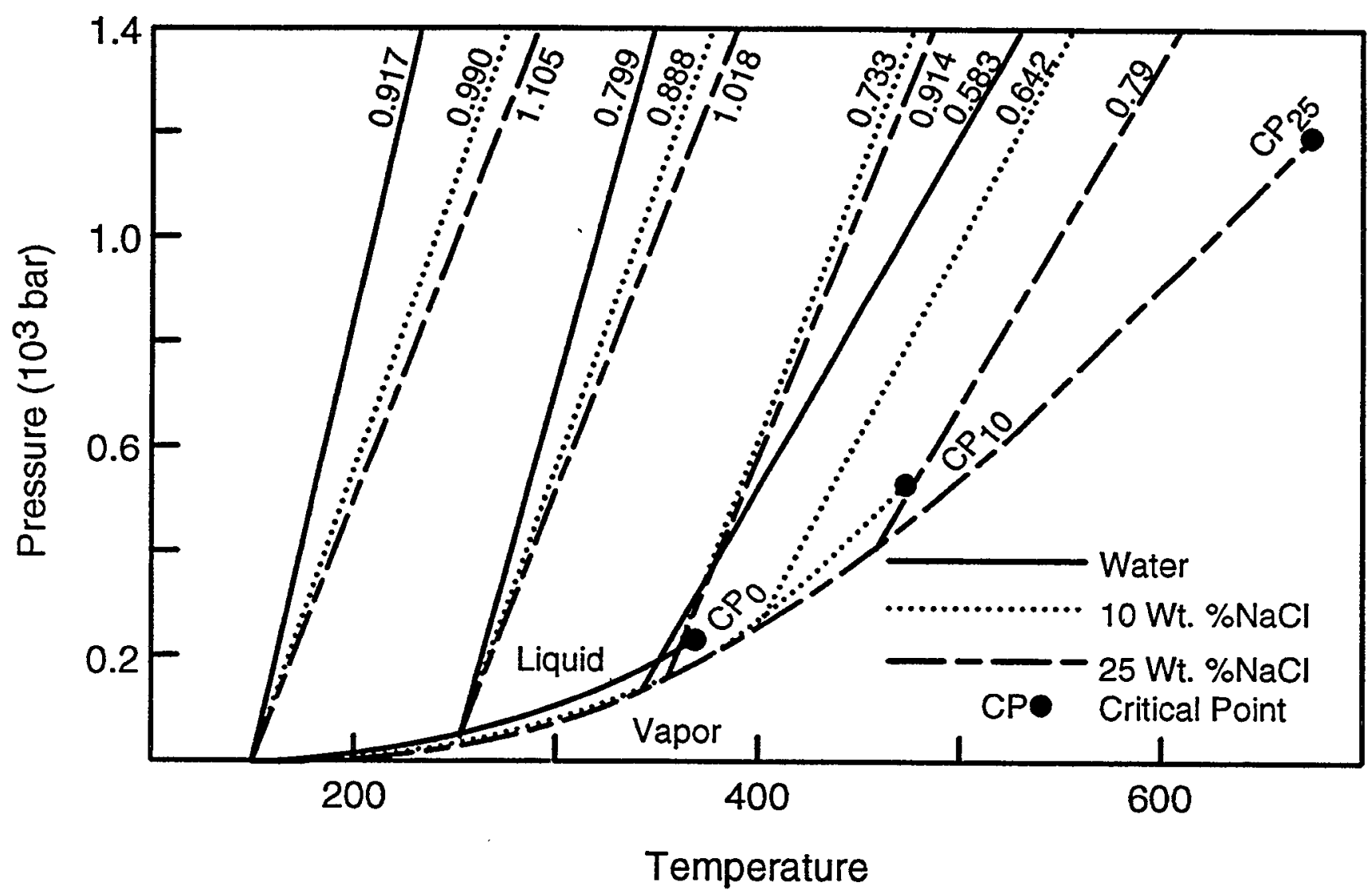

Figure 2-27. Shifting of the vapor-liquid line for pure water (solid line) with the addition of $10 \mathrm{wt} \%$ $\mathrm{NaCl}$ (dotted line) and $25 \mathrm{wt} \% \mathrm{NaCl}$ (dashed line). The critical points are also shown. The straight lines are isochores representing constant density fluids with P-T. The numbers represent the density in $\mathrm{g} / \mathrm{cm}^{3}$ (adapted from Roedder, 1984).

couple and solution alkalinity, also serves to control gas partial pressures. The $\mathrm{CO}_{2}$ content of gases in the partially-saturated zone at Yucca Mountain exhibits little variation with respect to either time or space (Thorstenson et al., 1989), ranging up to about 1.6 percent $\mathrm{CO}_{2}$ by volume. Gas in equilibrium with J-13 water from hydrologically-saturated Topopah Spring tuff is approximately 1.5 percent $\mathrm{CO}_{2}$ by volume, and the $\mathrm{CO}_{2}$ content of air is about 0.03 percent. Several studies (Takenouchi and Kennedy, 1964, 1965; Bowers and Helgeson, 1983) indicate the importance of $\mathrm{CO}_{2}$ in the $\mathrm{H}_{2} \mathrm{O}-\mathrm{CO}_{2}-\mathrm{NaCl}$ system (Figure 2-28). At low pressures, however, the shift in the liquid-vapor curve is negligible. Because of the relatively large amount of water present in the Yucca Mountain system (40-70 percent saturated), gas composition is likely to be chemically saturated with water. As a result of the low $\mathrm{CO}_{2}$ gas concentrations and low gas pressures anticipated at Yucca Mountain, the effect of variations in $\mathrm{CO}_{2}$ on gas thermal properties is likely to be negligible.

The effects of mineral redistribution on fractured tuff permeability has been discussed in Section 2.10.2 with respect to fracture healing. An additional potential effect is on medium porosity and permeability due to electrically-charged swelling clays (also see Section 2.7.2). In the presence of poorly hydrolyzed cations such as $\mathrm{Na}^{+}$, the electrical double layer (EDL) around charged particles can cause the 


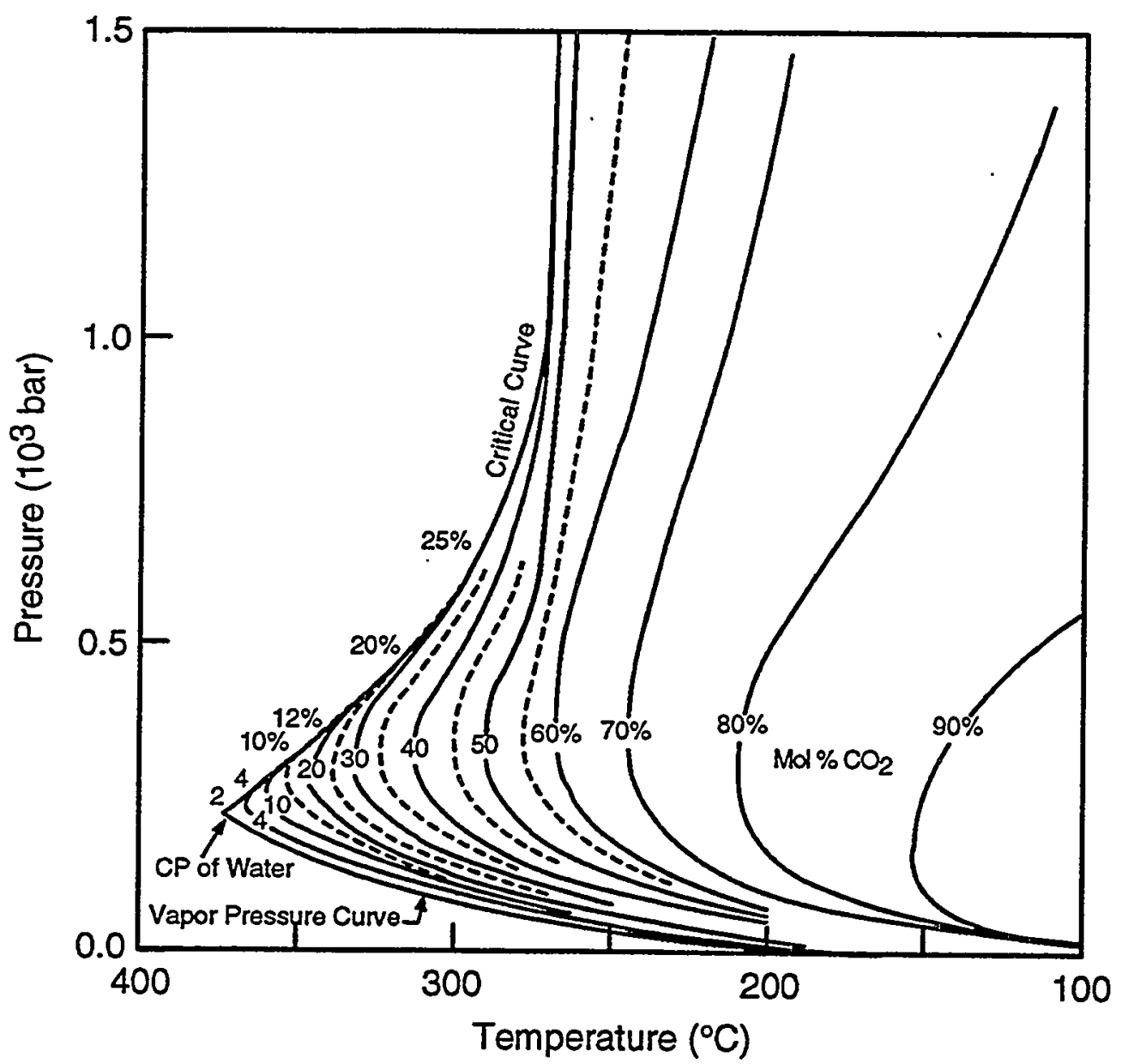

Figure 2-28. P-T plot of isocompositional curves for the gas phase in equilibrium with the liquid phase for the system $\mathrm{H}_{2} \mathrm{O}-\mathrm{CO}_{2}$. The critical curve for the system extends from the critical point for water $\left(374^{\circ} \mathrm{C}\right.$ and 220 bars) to lower temperatures and higher pressures. The minimum critical temperature occurs at approximately $266^{\circ} \mathrm{C}$ and about 2150 bars (adapted from Takenouchi and Kennedy, 1964). 
expansion of swelling clays such as smectite, and also tends to promote clay dispersion. This in turn clogs pores with fine particles, shifting the pore-size distribution to smaller-diameter pores, and reduces the permeability, hydraulic conductivity, and water diffusivity (Siyag et al., 1983; Nielsen et al., 1986) of the medium (Figure 2-29). Called the salinity effect by Nielsen et al. (1986), this phenomenon has been documented in numerous studies of partially-saturated soils under arid and semi-arid conditions (Suarez et al., 1984; Russo, 1989b; Goldberg et al., 1991): Even under saturated conditions, the hydraulic conductivity can be reduced by an order of magnitude or more (Laryea et al., 1982; Nielsen et al., 1986). Brandl (1981) observed that, with the addition of portlandite $\left[\mathrm{Ca}(\mathrm{OH})_{2}\right]$ to soil aggregates, permeabilities initially increased, before decreasing with curing age. It seems that poorly-hydrolyzed $\mathrm{Ca}^{++}$initially reduces swelling of clays, "unclogging" pores. As time passes, however, the cement begins to harden, filling voids and reducing permeability once again.

Variations in overall solution chemistry and moisture content also influence the magnitude of the salinity effect. Suarez et al. (1984) noted decreasing hydraulic conductivities with increasing $\mathrm{pH}$. This is likely due to the fact that at low $\mathrm{pH}$, the surface charge is high, and edge-surface bonding of clays, as well as bonding of positively charged oxides to negatively charged clay surfaces, occurs. Under these conditions, clay dispersion is low, and the adverse effect on hydraulic conductivity is minimal. As the $\mathrm{pH}$ increases, the surface charge decreases, clay dispersion is enhanced, and the salinity effect is amplified (Suarez et al., 1984). In a similar manner, at low ionic strengths or with predominantly monovalent cations, the EDL expands outward from particle surfaces into the pores, leading to a more pronounced salinity effect with decreasing electrolyte concentration (Nielsen et al., 1986). Finally, as the moisture content decreases, the water film becomes thinner, and the thickness of the EDL becomes relatively more important. Under drying conditions, reductions in water diffusivity and hydraulic conductivity due to the salinity effect become more pronounced, with decreases of as much as 3 orders of magnitude, as the moisture content declines from $0.5 \mathrm{~cm}^{3} / \mathrm{cm}^{3}$ to $0.3 \mathrm{~cm}^{3} / \mathrm{cm}^{3}$ (Siyag et al., 1983; Laryea et al., 1982; Conca, 1990).

Most of these studies have been conducted in soils, and the extent to which the treatment is applicable to intact rock, such as the volcanic tuffs at Yucca Mountain, is very uncertain. However, expandable smectitic clays are present as alteration products of the various tuff units (Broxton et al., 1987), and possibly as colloidal particles as well (Means et al., 1983). Water chemistries in the vicinity of Yucca Mountain indicate a sodium bicarbonate solution at low ionic strengths (Kerrisk, 1987). Given the arid climate of southern Nevada, it is possible that a correlation between solution chemistry and hydraulic properties of the geologic media, similar to that outlined above for soils, may exist at Yucca Mountain.

\subsubsection{Computer Codes}

Computer codes typically do not model only $\mathrm{C} \Rightarrow \mathrm{H}$ coupled processes. Many of the codes that model $\mathrm{H} \Rightarrow \mathrm{C}$ can model $\mathrm{C} \Rightarrow \mathrm{H}$. These computer codes are discussed in Section 2.9.3, and the discussion is not repeated here.

\subsubsection{Summary and Conclusions}

The coupling mechanisms for chemical to hydrologic $(\mathrm{C} \Rightarrow \mathrm{H})$ processes have been reviewed in this section. In total, four mechanisms were discussed: mineral deposition on fracture surfaces, fracture healing, chemical osmosis, and fluid properties. The mineralogy of the fracture surfaces can be important 


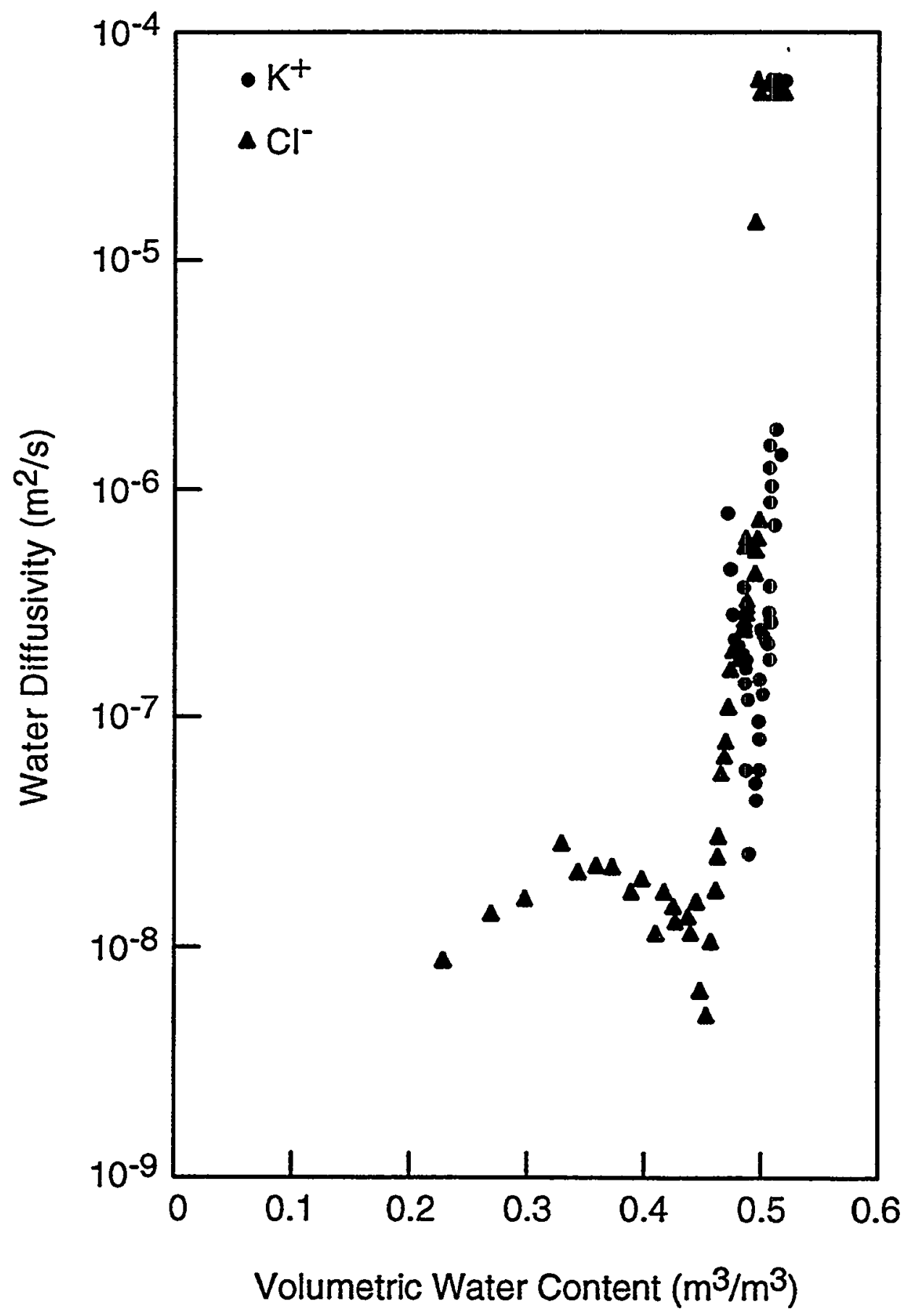

Figure 2-29. Water diffusivity of $\mathrm{K}^{+}$and $\mathrm{Cl}^{-}$as a function of volumetric water content for a silty clay loam (adapted from Laryea et al., 1982). 
in the imbibition of liquid into the matrix, hence influences the applicability of the effective continuum model. Fracture healing has been experimentally observed where minerals precipitate in fractures, thereby influencing fracture apertures and hydraulic conductivity. Chemical osmosis is a mechanism where liquid flows due to a dissolved species concentration gradient. Chemical composition can also influence fluid properties such as density, viscosity, and vapor pressure.

Each of these coupling mechanisms appears relevant to Yucca Mountain, however, none appears significant. Probably the most important coupling mechanism is the mineralogy of fracture surfaces, because this influences the distance liquid drains in a fracture before being imbibed into the matrix. Fracture healing may also be important in the near field as "healing" fractures may shield waste containers from fracture flow; however, this influence may be negligible in comparison with uncertainties associated with the surrounding rock (hydraulic conductivity, heterogeneities, fracture locations, etc). In summary, the effect of chemistry on fracture surfaces and apertures is considered potentially important, and warrants further consideration for the proposed repository.

\subsection{MECHANICAL $\Rightarrow$ CHEMICAL}

Mechanical stresses in the repository environment will affect the chemistry of the system mostly through pressure changes in the system. The effects of pressure on chemical equilibrium are well established and can be calculated through thermodynamics. The effects of pressure on nonequilibrium processes are less well understood, but an assumption of partial equilibrium can allow for modeling reaction progress as a function of pressure. Because the repository will be constructed in the partiallysaturated zone at Yucca Mountain, gas pressure at the repository is expected to be close to $1 \mathrm{~atm}$, and pressure effects are likely to be overshadowed by temperature effects, as discussed in Sections 2.5 and 2.6.

It is also possible that the chemistry will be perturbed by the construction of the repository itself. Fracturing of the rock can expose fresh, reactive surfaces to the fluids, enhancing reaction rates. Opening new pathways can alter gas flow, potentially leading to large changes in the partial pressures of critical gases such as ' $\mathrm{CO}_{2}$ and $\mathrm{O}_{2}$ (Murphy, 1990).

\subsubsection{Pressure Effects on Chemical Equilibrium}

is

At constant temperature, the key relationship between the free energy of reaction and pressure

$$
\left(\frac{\partial \Delta \mathrm{G}_{\mathrm{R}}}{\partial \mathrm{P}}\right)_{\mathrm{T}}=\Delta \mathrm{V}_{\mathrm{R}}=\Sigma \mathrm{V}_{\text {products }}-\Sigma \mathrm{V}_{\text {reactants }}
$$

where

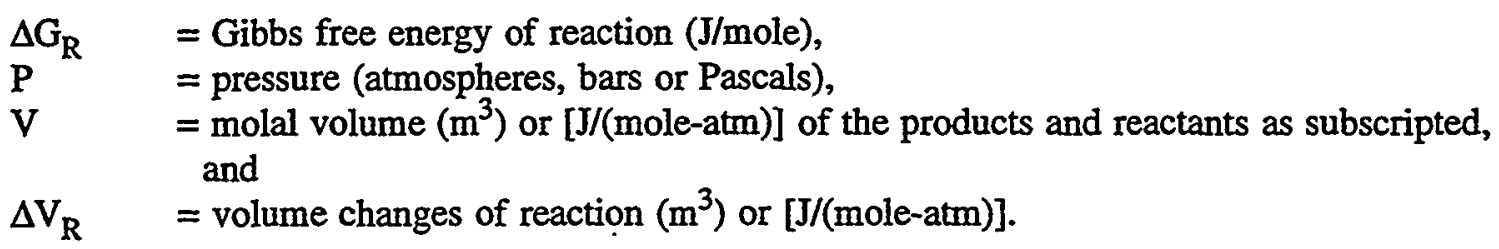


For a given temperature, $T$, and assuming a reference pressure of $1 \mathrm{~atm}$, integration of Eq. (2-37) results in

$$
\Delta \mathrm{G}_{\mathrm{R}, \mathrm{T}, \mathrm{P}}=\Delta \mathrm{G}_{\mathrm{R}, \mathrm{T}, 1}^{\mathrm{o}}+\int_{1}^{\mathrm{P}} \Delta \mathrm{V}_{\mathrm{R}} \mathrm{dP}
$$

As written in Eq. (2-38), $\Delta \mathrm{V}_{\mathrm{R}}$ is dependent on pressure. For solids and liquids, molal volume as a function of pressure is represented by the coefficient of isothermal compressibility, $\beta_{I}\left(a \mathrm{~atm}^{-1}\right.$ or $\mathrm{m}^{2} \mathrm{~N}$ ), such that

$$
\beta_{I}=-\frac{1}{V}\left(\frac{\partial V}{\partial P}\right)_{T}
$$

Molal volumes can also be expressed as a polynomial function of pressure (Richardson and McSween, 1989), which can be readily integrated.

If $\Delta V_{R}$ is independent of pressure, it is treated as a constant, and the integration of Eqs. (2-37) and (2-38) is greatly simplified. Solids are relatively incompressible (i.e., $\beta_{I}$ is very small), and volume changes with pressure are slight. At relatively-low pressures (tens of atmospheres) in the absence of a gas phase, liquids are also generally assumed to be incompressible. For this reason, $\Delta V_{R}$ is commonly assumed to only be constant for chemical reactions involving solids and liquids. For a constant $\Delta V_{R}$, combining Eq. (2-38) with the equilibrium relationship [Eq. (D-6)] $\Delta G_{R}{ }^{0}=-R_{I} T[\ln (K)]$ yields the relationship

$$
\ln \frac{\mathrm{K}_{\mathrm{P}_{2}}}{\mathrm{~K}_{1}}=-\frac{\Delta \mathrm{V}_{\mathrm{R}}\left(\mathrm{P}_{2}-1\right)}{\mathrm{R}_{\mathrm{I}} \mathrm{T}}
$$

At constant temperature and electrolyte concentration (molality, $\mathrm{m}_{\mathrm{I}}$ ) activity coefficients, $\gamma_{\mathrm{I}}$, vary as a function of pressure according to the relationship

$$
\left(\frac{\partial \ln \gamma_{\mathrm{I}}}{\partial \mathrm{P}}\right)_{\mathrm{T}, \mathrm{m}}=\frac{\mathrm{V}_{\mathrm{I}}-\mathrm{V}_{\mathrm{I}}^{\mathrm{o}}}{\mathrm{R}_{\mathrm{I}} \mathrm{T}}
$$

where

$$
\begin{array}{ll}
\mathrm{V}_{\mathrm{I}} & =\text { partial molar volume of species } \mathrm{i} \text { under actual conditions }\left(\mathrm{m}^{3}\right) \text { or }[\mathrm{J} /(\mathrm{mole}-\mathrm{atm})], \\
& \text { and } \\
\mathrm{V}_{\mathrm{I}}^{\circ} & =\text { partial molal volume under defined standard-state conditions }\left(\mathrm{m}^{3}\right) \text { or }[\mathrm{J} /(\mathrm{mole}-\mathrm{atm})] .
\end{array}
$$

Because partial molar volumes of dissolved species are a function of ionic strength, Stumm and Morgan (1981) point out that pressure effects on equilibrium constants will differ for dilute aqueous solutions as compared to high-ionic-strength solutions.

In contrast to solids and liquids, changes in total pressure can result in significant changes in gas molal volumes and partial pressures, and therefore in chemical reactions involving gas phases. According to the Ideal Gas Law, pressure $(P)$ and volume $(V)$ are related such that 


$$
P V=n R_{I} T
$$

where

$$
\begin{aligned}
& \mathrm{R}_{\mathrm{I}}=\text { ideal gas constant }[\mathrm{J} /(\mathrm{mol} \cdot \mathrm{k})] \\
& \mathrm{T}=\text { absolute temperature }(\mathrm{K}), \text { and } \\
& \mathrm{n}=\text { number of moles. }
\end{aligned}
$$

For one mole of gas, $\mathrm{PV} / \mathrm{R}_{\mathrm{I}} \mathrm{T}=1$ indicates that the gas behaves ideally. At temperatures of interest and at pressures up to $1 \mathrm{~atm}$, most gases behave ideally and no additional corrections are necessary. For pressures between one and several hundred atmospheres, $P V / R_{\mathrm{I}} \mathrm{T}<1$ for many gases, indicating the gas is more compressible than would be expected. This is likely to be due to attractive forces between the molecules which reduce the effective volume of the gas mixture. An empirical relationship called the van der Waals equation (e.g., Richardson and McSween, 1989) has been developed to account for this deviation from ideality such that

$$
\left[P+\left(\frac{a_{V D W}}{V^{2}}\right)\right]\left(V-b_{V D W}\right)=R_{I} T
$$

where

$$
\begin{array}{ll}
a_{\mathrm{VDW}} & =\text { an empirical factor determined for each gas }\left[\mathrm{J}^{2} /\left(\mathrm{mole}^{2}-\mathrm{atm}\right)\right], \text { and } \\
\mathrm{b}_{\mathrm{VDW}} & =\text { an empirical factor determined for each gas }[\mathrm{J} /(\text { mole-atm })]
\end{array}
$$

In an ideal gas mixture, the partial pressure of an ideal gas is defined as the pressure that the gas would exert if it occupied the same volume alone (e.g., Freeze and Cherry, 1979). The total pressure of the ideal gas mixture is therefore equal to the sum of the component partial pressures. For gases B, C, and $\mathrm{D}$ interacting according to the reaction $\mathrm{bB}+\mathrm{cC}=\mathrm{dD}$, the equilibrium constant $\mathrm{K}$ is defined such that

$$
K=\frac{\left(f_{D}\right)^{d}}{\left(f_{B}\right)^{b}\left(f_{C}\right)^{c}}
$$

where $f_{I}^{i}=$ fugacity of the gas, $I$, raised to the reaction coefficient, $i$.

Fugacity is related to the partial pressure of the gas by assuming a standard state fugacity, and through the use of a fugacity coefficient, $\gamma_{f}$, such that $f_{I}=\gamma_{f J} p(I)$. A standard-state pressure of 1 atm is commonly chosen. As the gas mixture approaches ideal behavior, $\gamma_{f, I} \rightarrow 1$. Over a wide range in temperature $\gamma_{\mathrm{f}, \mathrm{I}} \approx 1$ for most real gases at pressures near 1 atm. In a similar fashion, most real gas mixtures exhibit ideal behavior for pressures between 0 and 1 atm and the fugacity of each gas in the mixture is approximately equal to its partial pressure. In the partially-saturated zone at Yucca Mountain, total gas pressures of $1 \mathrm{~atm}$ are anticipated, and an assumption of ideal behavior is reasonable. 


\subsubsection{Pressure Effects on Nonequilibrium Chemistry}

As mentioned in the introduction, the pressure effects on reaction rates are less clearly understood. If a reaction progress approach assuming local equilibrium (see Appendix D) is used, the development outlined above is appropriate. Transition-state theory (Stumm and Morgan, 1981; Lasaga, 1981b; Aagaard and Helgeson, 1982) uses a modified form of Eq. (2-40) to express the effect of pressure on the rate constant $(k)$ for the formation of an activated complex (e.g., $X_{A C T}$ ) such that

$$
\left[\frac{\partial \ln \left(\mathrm{k}_{\mathrm{r}}\right)}{\partial \mathrm{P}}\right]_{\mathrm{T}, \mathrm{m}}=-\frac{\Delta \mathrm{V}_{\mathrm{ACT}}}{\mathrm{R}_{\mathrm{I}} \mathrm{T}}
$$

where the volume of activation $\Delta \mathrm{V}_{\mathrm{ACT}}=\mathrm{V}_{\mathrm{ACT}}-\left(\mathrm{V}_{\mathrm{A}}+\mathrm{V}_{\mathrm{B}}\right)$, for $\mathrm{A}+\mathrm{B} \rightarrow \mathrm{X}_{\mathrm{ACT}}$. Assuming a standard state of 1 atm and that $\Delta \mathrm{V}_{\mathrm{ACT}}$ is constant with pressure, integrating Eq. (2-45) yields a form similar to Eq. (2-40) such that

$$
\ln \frac{\mathrm{k}_{\mathrm{r}, \mathrm{P}_{2}}}{\mathrm{k}_{\mathrm{r}, 1}}=-\frac{\Delta \mathrm{V}_{\mathrm{ACT}}\left(\mathrm{P}_{2}-1\right)}{\mathrm{R}_{\mathrm{I}} \mathrm{T}}
$$

From Eq. (2-46), a plot of $\ln \mathrm{k}_{\mathrm{T}, \mathrm{P}_{2}}$ versus $\mathrm{P}$ yields $\Delta \mathrm{V}_{\mathrm{ACT}}$. If $\Delta \mathrm{V}_{\mathrm{ACT}}<0$, then the rate constant increases with pressure.

\subsubsection{Pressure Solution}

Mineral dissolution in response to stresses at grain-grain contacts, and the mobilization and reprecipitation of chemical species in response to the resultant chemical gradients, are observed in many types of diagenetic and metamorphic environments. Dewers and Ortoleva (1990a) noted that this type of "stress-driven water-rock interaction" is divided into two distinct classes: (i) pervasive intergranular pressure solution (IPS), and (ii) the development of localized textures called stylolites. Two types of mechanisms are discussed by Dewers and Ortoleva (1990a). Water-film diffusion (WFD) involves dissolution in response to variations in normal stress across contacts; chemicals diffuse down the chemical potential gradients along the contact in a thin water film adsorbed to the grain surface. Free-face pressure solution (FFPS) includes dissolution stimulated by elastic strain energy at contact asperities and possible increases in surface area resulting from crushing at the microscale.

Pervasive IPS tends to remove textural heterogeneities through time. As the dissolution reaction proceeds under stress, the grain-grain contact increases in area. Small area point contacts tend to redistribute faster, and larger areas tend to react slower, resulting in a relatively narrow spatial average. WFD tends to favor this process. Conversely, FFPS tends to favor the preservation of small area asperities, maintaining and enhancing existing textural heterogeneities in the medium, and promoting the development of stylolites. Rates of FFPS are limited by diffusion at grain contacts. Faster rates of reaction tend to minimize stylolite development.

In a series of recent papers (Ortoleva et al., 1987; Dewers and Ortoleva, 1990a,b), a coupled $\mathrm{M} \Rightarrow \mathrm{C}$ model was developed to simulate the growth of stylolites during the burial and compaction of sandstones. A representative element volume was assumed to be made up of a large number of identical 
grains (truncated spheres) arranged in a regular array. The model used three types of contact surfaces and a free face area, and considered WDS and FFPS to be the only mechanisms contributing to pressure solution. Pressure normal to the contact is different for the four different surface types. Solute transport is only by diffusion. The pressures and times considered are generally greater $(0-3 \mathrm{~km}$ depth of burial, and $0-550$ million yrs) than those of interest in a HLW repository.

\subsubsection{Computer Codes}

As is the case with temperature effects, geochemical codes are not generally coupled to mechanical processes in the sense of directly simulating the effects of stress on chemistry. Pressure effects can be simulated, however, though the number of geochemical codes that can extrapolate beyond $1 \mathrm{~atm}$ is much more limited than those that can consider thermochemical processes. Below is a brief listing of those codes that can take into account pressure variations. The codes are discussed in more detail in Section 2.6. More complete listings are available in other studies (Nordstrom and Ball, 1984; Nordstrom and Munoz, 1985; Morrey et al., 1986; Serne et al., 1990; Mangold and Tsang, 1991).

\subsubsection{SUPCRT}

As discussed in Section 2.5.3.1, SUPCRT is able to calculate equilibrium constants as a function of pressure variations. The thermodynamic data are internally consistent (Helgeson et al., 1978) and extrapolation to 5000 bars and $1000^{\circ} \mathrm{C}$ is possible in the most recent version (Johnson et al., 1991).

\subsubsection{EQ3/6}

The EQ3/6 code is able to model pressure variations in a somewhat limited fashion. Below $100^{\circ} \mathrm{C}$, the code is limited to $1 \mathrm{~atm}$. Above $100^{\circ} \mathrm{C}$, the pressure variation with temperature is constrained to follow the liquid-vapor saturation curve for the pure- $\mathrm{H}_{2} \mathrm{O}$ system. For this reason, the code is limited to a single, fixed pressure for a given temperature, and a maximum pressure of 220 bars at the critical point of $374^{\circ} \mathrm{C}$ (Roedder, 1984).

\subsubsection{Summary and Conclusions}

The equations governing the effect of pressure on chemical processes are presented and discussed. Liquids and solids are generally considered incompressible (no volume change) except at very high pressures. Gases are strongly affected by pressure, and the ideal gas law is used to describe gas behavior. An assumption of ideality is typically adequate at low pressures on the order of $1 \mathrm{~atm}$ or less. Deviations from ideality are described using the empirical van der Waals equation. Pressure also influences reaction rate constants. At the gas pressures anticipated in the proposed repository, however, these effects are minimal, and an assumption of ideality is probably adequate. Pressure solution is discussed, but times and pressures of interest in the repository environment are significantly less than those for which pressure solution has been modeled.

\subsection{CHEMICAL $\Rightarrow$ MECHANICAL}

The chemical effects of water and other aqueous environments can play an important role in the deformation and strength of rocks. Certain chemical environments have long been known to enhance the propagation of fractures in rock. For example, Dunning et al. (1980), and Westwood (1974) have shown 
that the presence of aqueous chemical environments results in a measurable, and sometimes substantial, weakening in single crystals of quartz and other geological materials. Atkinson (1979), Boozer et al. (1963), and Vutukuri (1974) have demonstrated that aqueous chemical environments also have a weakening effect on polycrystalline geologic materials. This weakening effect has been observed to be a result of the reduction in the surface free energy of the materials due to absorption of species from the chemical environments (Dunning et. al., 1980). It has also been thought that the zeta potential (surface electrostatic potential) also plays a role in chemomechanical weakening.

\subsubsection{Chemical Effects on Rock Fracture Propagation}

The potential role of surface free energy in chemomechanical weakening can be observed from the Griffith energy balance theory of crack propagation, as shown below (Griffith, 1920).

$$
\mathrm{U}=\left(\mathrm{W}_{\mathrm{L}}+\mathrm{U}_{\mathrm{e}}\right)+\mathrm{U}_{\mathrm{s}}
$$

where

$$
\begin{array}{ll}
W_{\mathrm{L}} & \text { = work done to displace the outer boundary of the material }(\mathrm{J}), \\
\mathrm{U}_{\mathrm{e}} & \text { = stored elastic strain energy }(\mathrm{J}), \\
\mathrm{U}_{\mathrm{S}} & \text { = surface free energy }(\mathrm{J}), \text { and } \\
\mathrm{U} & =\text { total energy of the system }(\mathrm{J}) .
\end{array}
$$

The crack system is said to be in equilibrium (i.e., neither being extended nor closed) when $d U / d c=0$, where $c$ is the crack length. Lawn and Wilshaw (1975) showed that the critical stress, $\sigma_{c}$, required to propagate the crack or flow is

$$
\sigma_{c}=\left[\frac{2 \mathrm{E} U_{s}}{\left(1-v_{\mathrm{P}}^{2}\right) \pi \mathrm{L}_{\mathrm{c}}}\right]^{1 / 2}
$$

where

$$
\begin{array}{ll}
\sigma_{\mathrm{c}} & =\text { critical stress }\left(\mathrm{N} / \mathrm{m}^{2}\right), \\
\mathrm{v}_{\mathrm{P}} & =\text { Poisson's ratio (dimensionless), } \\
\mathrm{E} & =\text { Young's modulus }\left(\mathrm{N} / \mathrm{m}^{2}\right), \\
\mathrm{U}_{\mathrm{S}} & =\text { surface free energy }(\mathrm{J}, \text { and } \\
\mathrm{L}_{\mathrm{c}} & =\text { crack length }(\mathrm{m}) .
\end{array}
$$

Equation (2-48) shows the dependency of the critical stress to the surface free energy. In other words, a reduction in the surface free energy would result in a reduction in the critical stress required to propagate the crack. The role of the zeta potential has been found to be more complex in its impact on crack propagation.

Dunning et al. (1984) conducted laboratory experiments on the effects of various aqueous chemical environments on crack propagation in quartz. Figure 2-30 shows the results of their experiments. The figure shows that the crack propagation stresses for the natural quartz decrease sharply from an ambient atmospheric environment to one containing water $\left(\mathrm{H}_{2} \mathrm{O}\right)$. As the chemical environment becomes progressively more cationic, the crack propagation stresses are seen to further decrease as a result of 


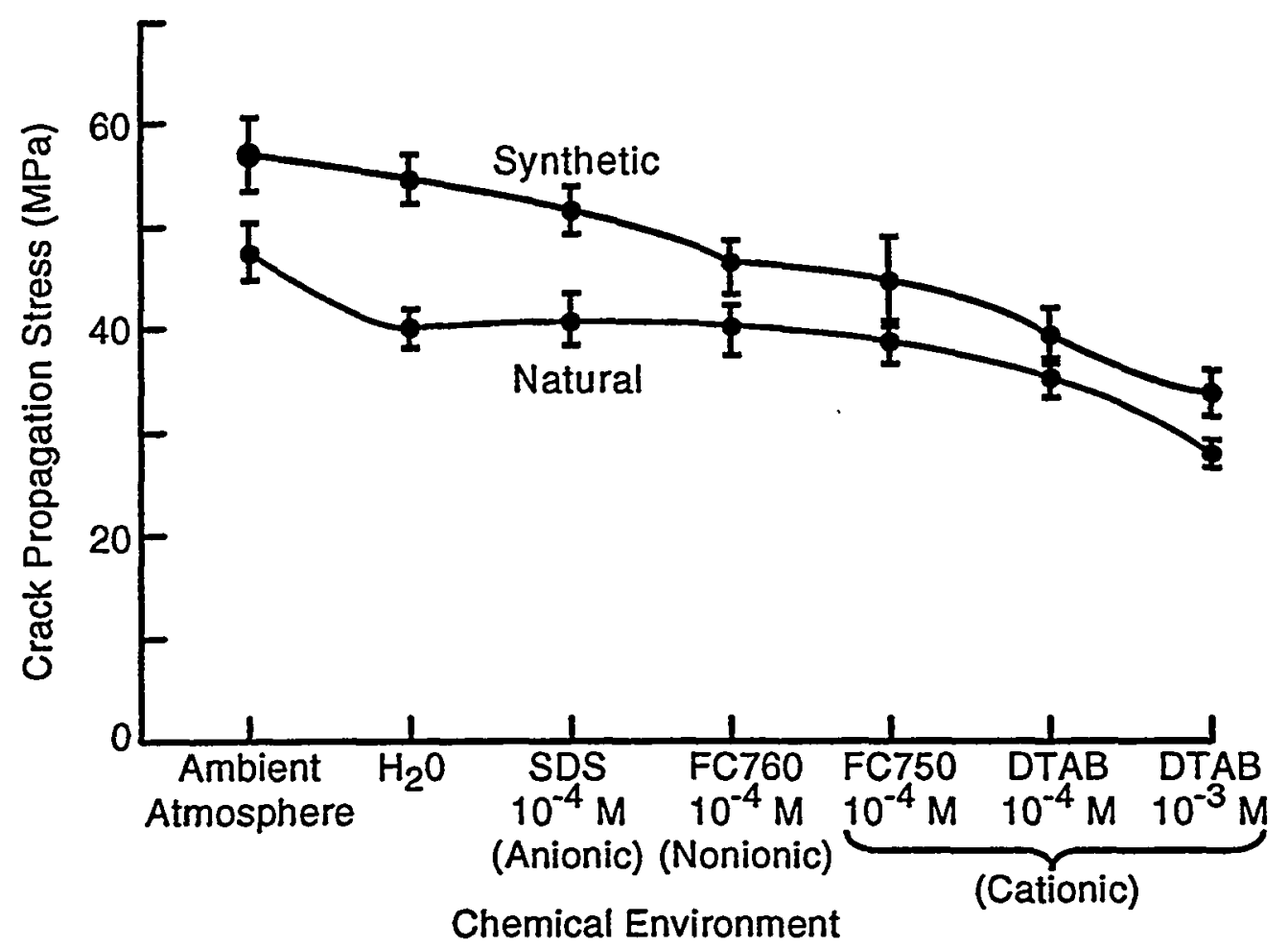

Figure 2-30. Crack propagation stress of synthetic and natural quartz samples plotted versus chemical environment (Dunning et al., 1984).

adsorption of the chemical species into the rock. They also found that the surface free energy more closely correlated with crack propagation stress than the square of the crack propagation stress, as indicated by Eq. (2-48). This apparent disparity with the Griffith energy balance concept could be due to the possibility that surface energy reduction is only one of several operating mechanisms of weakening.

\subsubsection{Degradation of Mechanical Properties Due to Chemical Action}

Mechanical properties of rock may be altered by chemical processes at the most basic level through dissolution/precipitation of minerals from solution or the replacement of one mineral by another during alteration (Helgeson, 1979; Stumm and Morgan, 1981; Henley et al., 1985; Murphy and Helgeson, 1987). An example of such a transformation is the hydrolysis of feldspars (minerals which make up 50 percent or more of the Topopah Spring tuff) to micas and clays such as muscovite and kaolinite. Although the dissolution of feldspar is incongruent, the overall alteration of feldspar to kaolinite in the presence of $\mathrm{CO}_{2}$ can be written in the general form:

$$
\underset{\text { (feldspar) }}{2 \mathrm{KAlSi}_{3} \mathrm{O}_{8}}+2 \mathrm{CO}_{2}(\mathrm{~g})+11 \mathrm{H}_{2} \mathrm{O}=\underset{\text { (kaolinite) }}{2 \mathrm{~K}^{+}}+2 \mathrm{HCO}_{3}^{-}+4 \mathrm{H}_{4} \mathrm{SiO}_{4}+\mathrm{Al}_{2} \mathrm{Si}_{2} \mathrm{O}_{5}(\mathrm{OH})_{4}
$$


Mineral reactions of this type are commonly observed in geothermal systems and in hydrothermally altered rock associated with ore deposits, and as secondary products of surface weathering (Rose and Burt, 1979; Henley et al., 1985). Broxton et al. (1987) identified clay alteration assemblages of illite, smectite, and/or chlorite below Yucca Mountain in the Crater Flat tuff. Bish (1986) used the illite/smectite transition to identify a greater than 10 million year paleohydrothermal system associated with the Timber Mountain Caldera. Based on illite/smectite chemistry, alteration temperatures were as low as $100^{\circ} \mathrm{C}$ at the southern end of Yucca Mountain.

Alteration minerals such as clays and micas have different physical properties from the minerals in fresh rock. Alteration minerals like clays, especially swelling clays such as smectite, are weaker than feldspars and can have a pronounced effect in decreasing the strength of the rock. For tuffs in the Yucca Mountain vicinity, a general decrease in Young's Modulus is observed for rocks containing a significant clay (smectite) component (DOE, 1988). Friction coefficients for clay-lined fractures are also generally less than those for fresh rock (DOE, 1988). Conversely, precipitation of silica (Rimstidt et al., 1989) can indurate rocks and increase rock strength.

Temperatures anticipated in association with the emplacement of HLW are high enough for significant alteration (up to $200{ }^{\circ} \mathrm{C}$, see Figure A-2). In the absence of water, however, hydrolysis reactions will not occur. The drying out of the near-field due to waste emplacement can serve to reduce the extent of alteration. As discussed in Section 2.1, flow through fractures is possible, and clay alteration may occur along fracture surfaces.

\subsubsection{Stress Corrosion Cracking}

Stress corrosion cracking (SCC) has been discussed for minerals and rocks (Atkinson and Meredith, 1987) as well as for glass, ceramics, and metals (Jones, 1992; Jones and Ricker, 1992). The significance of SCC in geologic materials appears uncertain because of the wide range of variables encountered. For the engineered barrier system of a repository, however, SCC has been identified as a potentially-important failure mechanism for waste packages (DOE, 1988; Cragnolino and Sridhar, 1991, 1992).

Metals exposed to certain aqueous solutions while under a tensile stress, may be subject to crack initiation and propagation, leading to premature failure through SCC. Once initiated, SCC can occur fairly quickly, with crack velocities ranging from $10^{-12}$ to $10^{-3} \mathrm{~m} / \mathrm{s}$ (Macdonald and Cragnolino, 1989). Two different modes of cracking occur; transgranular cracks run across the grain, and intergranular cracks propagate along grain boundaries. As discussed by Jones and Ricker (1992), SCC mechanisms include, among others: (i) pre-existing active-path mechanisms, (ii) absorption-related mechanisms, and (iii) strainassisted active-path mechanisms. Intergranular SCC along reactive grain boundaries is an example of the first type. Reduction of $\mathrm{H}^{+}$ions to adsorbed hydrogen atoms, followed by incorporation of atomic hydrogen into the metal lattice and the resultant embrittlement of the metal, is an example of type (ii). As an example of strain-assisted, active-path SCC, transgranular SCC proceeds as a series of steps that involve the rupture of a protective passive film by straining, anodic localized dissolution of the metal at the crack tip, and repassivation of the crack walls.

The rate and extent of SCC depends on the alloy being considered, the amount of stress involved, and the chemical environment in which the metal is placed. Stresses can be provided either through external means such as bending, or be due to residual internal stresses resulting from manufacture, 
forming, and machining of the metal. As in many alloy/environment systems, the time-to-failure for several stainless steels in boiling concentrated $\mathrm{MgCl}_{2}$ brines ( 42 percent $\mathrm{MgCl}_{2}$ at $154{ }^{\circ} \mathrm{C}$ ) increases with decreasing applied stress (Figure 2-31). For each steel, there exists a threshold value, ranging from 10 to 100 percent of the 0.2 percent yield strength, below which SCC does not occur. Crack growth rates can be expressed in terms of a stress intensity factor, $\mathrm{K}_{\mathrm{T}}$, which is proportional to the applied stress and to the square root of the crack length (Figure 2-32). For low values of the crack growth rate, the crack growth rate is dependent on $\mathrm{K}_{\mathrm{I}}$. At high values, the crack propagation rate is independent of $\mathrm{K}_{\mathrm{I}}$. The upper limit is the critical value, $\mathrm{K}_{\mathrm{IC}}$, where crack growth due to mechanical overloading is observed in inert environments (Macdonald and Cragnolino, 1989). Below $\mathrm{K}_{\mathrm{IC}}$ is considered to be the region of SCC or subcritical crack growth. For stainless steels exposed at $250^{\circ} \mathrm{C}$ to a simulated boiling water reactor (BWR) environment (Figure 2-32), as well as for many other alloy/environment systems, a threshold stress intensity factor, $\mathrm{K}_{\mathrm{ISCC}}$, exists, below which crack growth is exceedingly slow $\left(<10^{-11} \mathrm{~m} / \mathrm{s}\right)$.

As can be seen from Figure 2-31, the resistance to $\mathrm{SCC}$ in a given environment is dependent on the specific alloy being considered. Figure 2-33 shows the effect of nickel content on the susceptibility to SCC of Fe-Cr-Ni alloys exposed to a boiling $\mathrm{MgCl}_{2}$ solution. For a constant applied stress, there is a significant decrease in failure time due to SCC for Ni-contents between about 5 and 30 percent. Above about 40 percent nickel, failure did not occur in 30 days. Farmer et al. (1988a,b) provided extensive surveys of SCC in stainless steels and copper-based alloys in support of the selection of HLW container materials. As pointed out by Macdonald and Cragnolino (1989), even small amounts of impurities or variations in alloy composition may result in significant differences in SCC susceptibility. Segregation of impurities, such as tin, phosphorous, and arsenic, during heat treatment may serve to enhance intergranular SCC of low-alloy steels. The surface condition also affects a metal's resistance to SCC. Scoring and grooves provide focus points for local enhanced stress, facilitating the initiation of SCC.

The chemical environment also controls susceptibility to SCC. Anodic dissolution at the crack tip is enhanced by the anion content of the solution. Cracking rates for stainless steel tend to increase with increasing chloride and oxygen contents of water (Farmer et al., 1988a). For SCC mechanisms (i) and (iii), crack growth rates are proportional to the anodic current density on a strained metal surface. In some cases, the metallic cation in solution is an indirect factor influencing cracking. Farmer et al. (1988a) noted that $\mathrm{Mg}^{2+}$ is most aggressive in attacking Type 304 stainless steel in water containing $100 \mathrm{ppm} \mathrm{Cl}^{-}$at $100{ }^{\circ} \mathrm{C}$, while $\mathrm{Na}^{+}$is the least aggressive. This is probably due to the fact that readily-hydrolyzable cations such as $\mathrm{Mg}^{2+}$ produce greater amounts of $\mathrm{H}^{+}$(and lower $\mathrm{pH}$ ) through hydrolysis than poorly-hydrolyzed cations such as $\mathrm{Na}^{+}$. The presence of species such as $\mathrm{H}_{2} \mathrm{~S}$ in the solution enhances cracking due to hydrogen embrittlement [mechanism (ii)]. Macdonald and Cragnolino (1989) noted that the electric potential difference established at the metal/water interface is the critical electrochemical factor controlling SCC. Below and above a certain potential range, which varies with metal/solution system type, repassivation of the metal occurs so rapidly that crack growth through dissolution is inhibited in the case of mechanisms (i) and (iii).

SCC is dependent on a tensile stress being applied to the metal. In the repository environment, it seems likely that most of the macroscopic stress applied to waste containers and metal components in the repository will be compressive. However, residual stresses from metal forming and tempering may provide the necessary tensile stress. Bending of metal components in response to compression produces tension on the outside of the bend. 


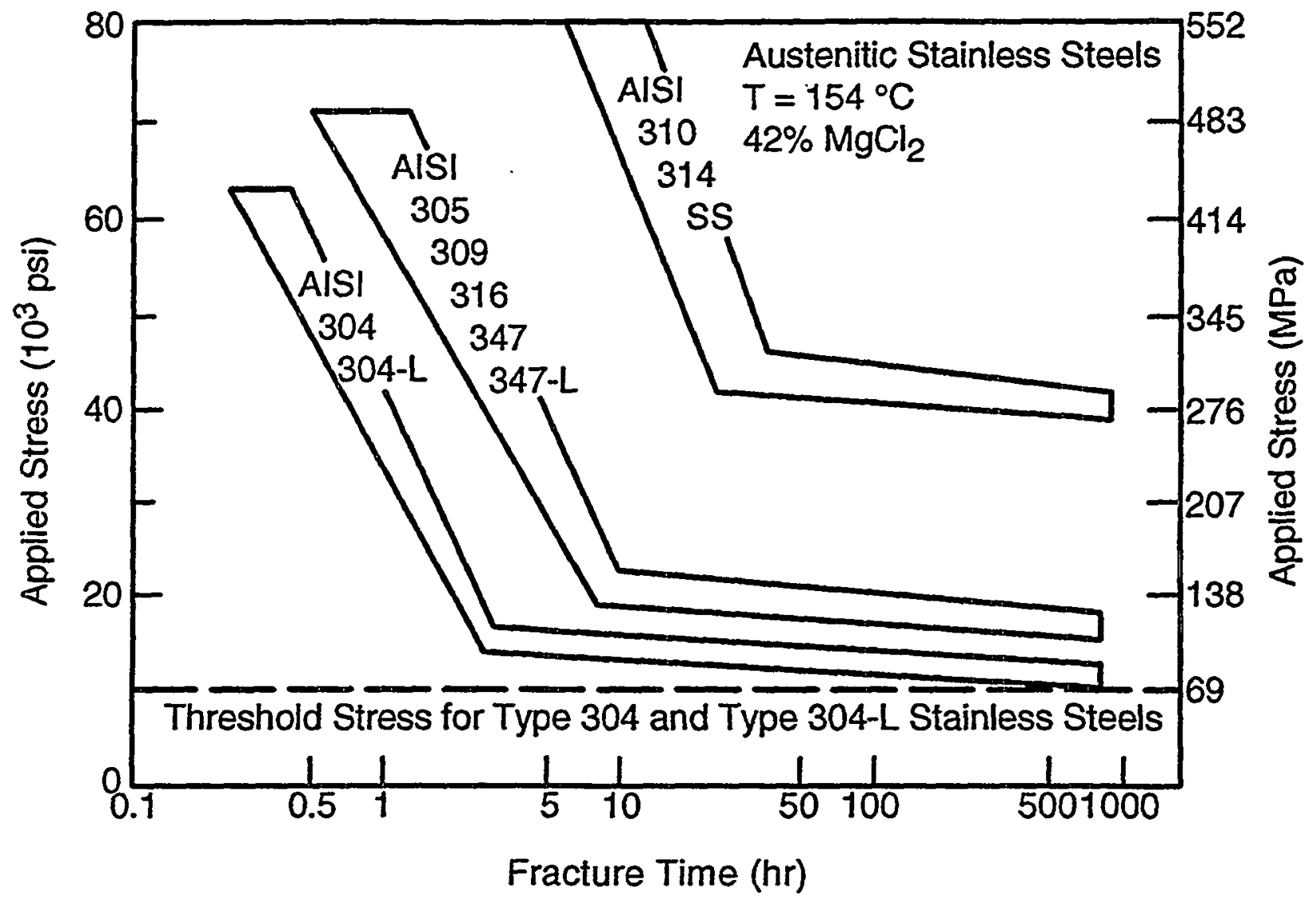

Figure 2-31. Resistance to SCC as a function of applied stress. Metals are commercial stainless steels in boiling $42 \% \mathrm{MgCl}_{2}$ at $154^{\circ} \mathrm{C}$ (adapted from Fontana and Greene, 1978).

\subsubsection{Computer Codes}

Computer codes typically do not model only $\mathrm{C} \Rightarrow \mathrm{M}$ coupled processes, but may include this capability in a $\mathrm{M} \Rightarrow \mathrm{C}$ computer code. These computer codes are discussed in Section 2.11 .4 , and the discussion is not repeated here.

\subsubsection{Summary and Conclusions}

The effect of chemical processes on mechanical processes has been discussed in this section. It has been noted that the crack propagation stress for a fracture under either shear or .tensile stresses can be substantially reduced depending on the chemical environment under which it exists. The section also discussed chemical processes in which certain minerals within the rock will be altered (e.g., alteration of feldspar to kaolinite). Such alteration of minerals can significantly lower the strength of the rock. However, due to the partially-saturated nature of the Yucca Mountain site and the drying out of the rock in the near-field due to waste emplacement, chemical processes may not become important on the mechanical state of the rock mass until well into the containment and isolation periods. SCC of metallic materials is also discussed. Because SCC is difficult to predict, and because the exact conditions anticipated at Yucca Mountain are imperfectly understood, much effort is currently under way to study 


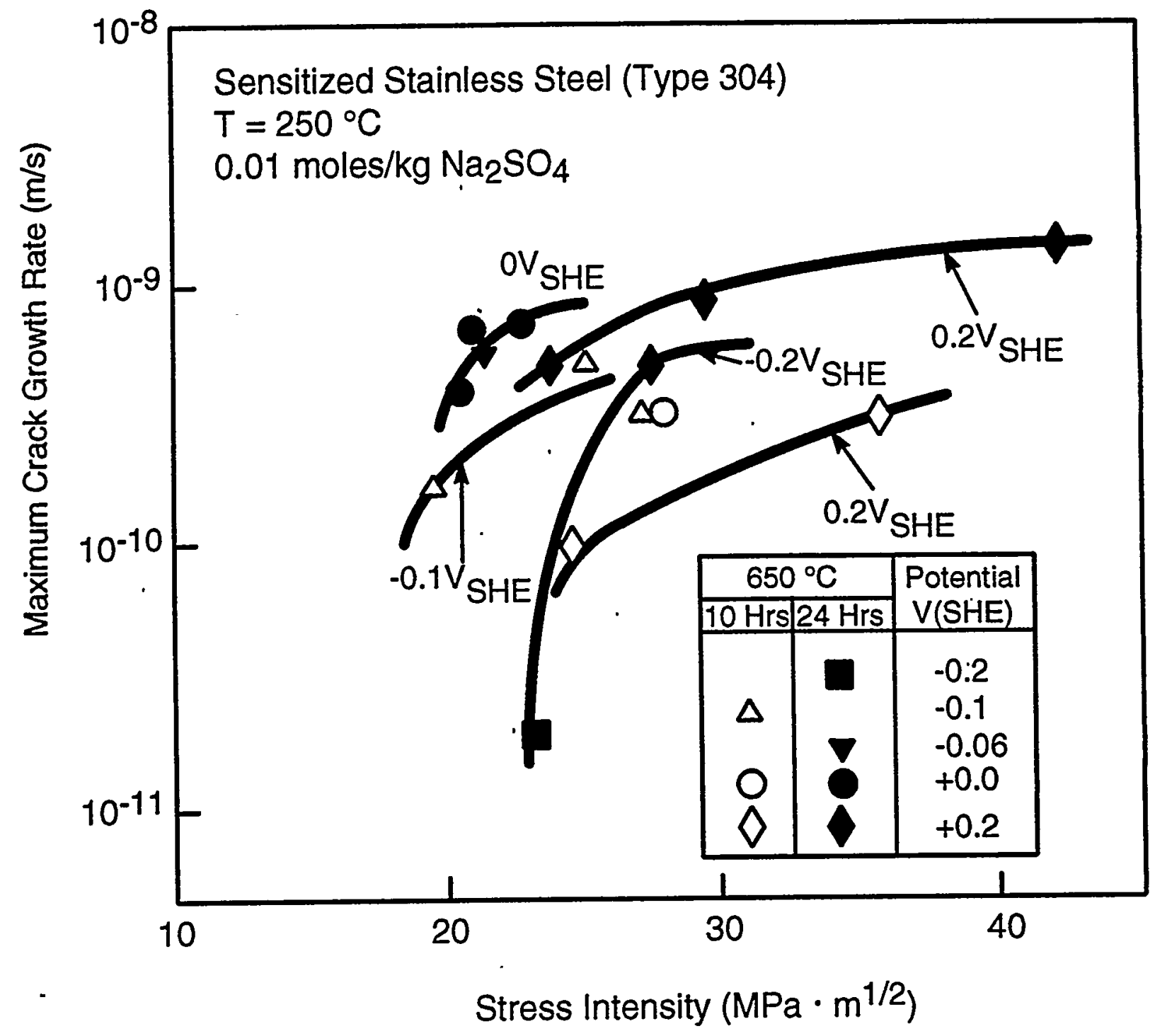

Figure 2-32. Crack growth rate $(\mathrm{m} / \mathrm{s})$ as a function of stress intensity $\left(\mathrm{MPa} \mathrm{m}^{1 / 2}\right.$ ) for Type 304 stainless steel (adapted from Chung et al., 1985). 


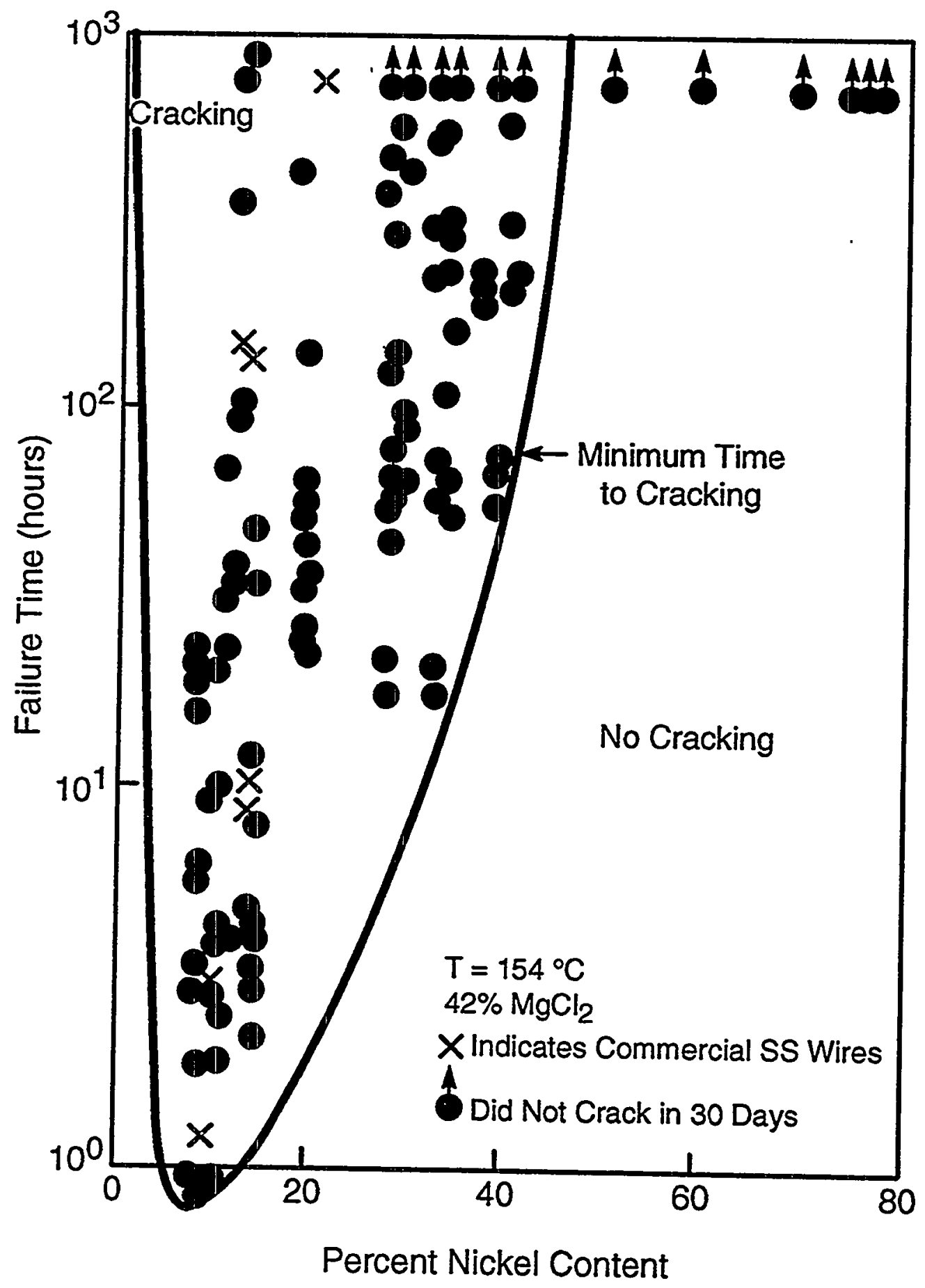

Figure 2-33. Susceptibility of Fe-18Cr-xNi alloys to $\mathrm{SCC}$ in boiling $42 \% \mathrm{MgCl}_{2}$ as a function of nickel content (adapted from Copson, 1959). 
SCC under likely repository conditions '(Cragnolino and Sridhar, 1992). Since container integrity is important to the design and performance of the repository, the $C \Rightarrow M$ coupling is considered potentially important for the proposed repository. 


\section{THREE-WAY COUPLED PROCESSES}

In this section, three-way couplings are considered. In total, four three-way couplings can be considered: TMH, THC, TMC, MHC. In Section 2, one-way coupling mechanisms are discussed, hence this chapter primarily synthesizes the results of Section 2. As a matter of presentation, the discussion in Section 2 has concentrated on possible coupling mechanisms, while this chapter concentrates on probable (or important) couplings. In addition, computer programs are emphasized in this chapter primarily out of necessity because the problems are complicated.

Because the material in this section is a synthesis of material in Section 2, and because certain couplings are more important than others, only two three-way couplings are explicitly discussed in this section: THM and THC. The TMC and HMC couplings have not received as much discussion in the literature. An assessment of the importance of different couplings is included in Section 4 of this report.

\subsection{THERMAL-HYDROLOGIC-MECHANICAL COUPLING}

The one-way couplings (e.g., $\mathrm{T} \Rightarrow \mathrm{H}, \mathrm{T} \Rightarrow \mathrm{M}, \mathrm{H} \Rightarrow \mathrm{M}$ ) discussed in Section 2.0 become further complicated when one considers the coupling between any three processes. Modeling these three-way couplings is made difficult by the fact that there is inexact knowledge of the couplings and governing equations. During radioactive decay of the HLW, the resulting temperature field will generate thermal stresses which produce normal and shear displacements of the fractures and compression or tension within the matrix. Heating of the groundwater in the vicinity of the repository produces vaporization, vapor flow, condensation, and condensate flow, hence the coupled system becomes one involving thermal, hydrological, and mechanical interactions (THM). An example of THM coupling is the effect of changing temperature and pore fluid pressure on mechanical changes of the rock fractures, such as those observed in hot dry rock experiments (Tsang, 1991).

The fundamental laws governing static equilibrium, fluid flow, and heat flow are coupled through the dependent variables of the solid displacement vector, fluid pressure, and medium temperature (Biot, 1941). These laws, in conjunction with constitutive equations, provide the approximate mathematical model of the coupled quasilinear THM phenomena in saturated porous elastic media. Additional extensions to the theory are necessary to simulate phenomena in porous fractured rocks and partially-saturated rock.

The complexity of the THM equations is such that an analytic (mathematical) solution for even simple initial- and boundary-value problems is not likely to be found. However, numerical solutions can be sought, if the mathematical model is good (Tsang, 1987b). Various numerical schemes using wellknown numerical techniques such as finite-element and finite-difference have been developed for THM modeling, and are discussed later in this section. Most of the computer codes for modeling coupled THM processes are only partially verified against existing analytical solutions for two-way couplings such as hydromechanical, thermomechanical, and hydrothermal problems. Also, in some computer codes the coupled equations are directly solved, whereas in other programs the THM coupling occurs in the solution process. For example, the solution process may involve running the thermal analysis out to some period of time, and then feeding the resulting temperatures into the mechanical routine to update the thermallyinduced stresses. Finally, the changes in the mechanical state will be used to update the hydrologic state.

Numerical simulation of the THM environment around a waste container or heater was conducted by Noorishad et al. (1984). The objective was to study the changes of fluid inflow to a heater borehole 
induced by the temperature rise. Figure 3-1(a) shows the 2D axisymmetric finite-element mesh which includes a horizontal fracture intersecting the bottom of the emplacement borehole. The fracture is assumed fully saturated, and extends out to a hydrostatic boundary at the edge of the mesh. Figure 3-1(b) shows the variation of fluid inflow to the heater borehole as a function of time. Before the heater raises the temperature of a large volume of the rock, the flow from the hydrostatic outer boundary to the borehole at atmospheric pressure is high. Later in time, with the heated rock above the fracture expanding and the fracture aperture near the heater borehole closing, the flow decreases sharply. Figure 3-2 shows the evolution of the fracture aperture profile, together with the variations of the pressure and temperature distributions. The pressure at 0 days represents the full hydrostatic pressure in the fracture before excavating the emplacement hole. The pressure diminishes rapidly after excavation and before major development of the thermal front. As thermal stresses are generated, causing closure of the fracture, the pressure again rises. After approximately 14 days, the pressure in the fracture again reaches its full hydrostatic value.

\subsubsection{DECOVALEX International Project}

An international project titled DECOVALEX (DEvelopment of COupled models and their VALidation against EXperiments in nuclear waste isolation) was begun in 1990, to increase the understanding of various THM processes of importance for radionuclide release and transport from a repository to the biosphere, and how they can be described by mathematical models. The main objectives of DECOVALEX are to:

- support the development of codes for THM modeling for jointed rocks;

- investigate and to apply suitable algorithms for THM modeling;

- investigate the capabilities of different codes to describe laboratory experiments and to perform verification of codes;

- compare theory and model calculations with experiments; and

- design validation experiments by means of THM model studies.

During the first phase of DECOVALEX, a series of benchmark and test-case problems have been designed to validate computer codes against each other as well as experimental data. One problem that has been used as a benchmark problem for DECOVALEX is shown in Figure 3-3(a). This benchmark test consisted of an assemblage of nine blocks, separated by two sets of planar fractures, one set being horizontal and the other vertical. The dimensions and locations of monitoring points are shown in Figure 3-3(a). The rock was subjected to in situ compressive stresses of $4.0 \mathrm{MPa}$ in both the horizontal and vertical directions. The mechanical boundary conditions consisted of zero normal displacements around the four edges of the model (i.e., boundaries were rollered). The rock matrix was assumed to be linear elastic, while the joint constitutive relations followed the Coulomb-friction model.

The hydraulic boundary conditions consisted of a fixed pressure of $10.0 \mathrm{kPa}$ on the left model boundary and a fixed pressure of $11.0 \mathrm{kPa}$ along the right model boundary. Both the top and bottom boundaries were assumed to be impermeable. The initial fluid pressure through the model was specified to be $10.0 \mathrm{kPa}$, and initial aperture for the joints under the in situ conditions was specified as 300 microns. 


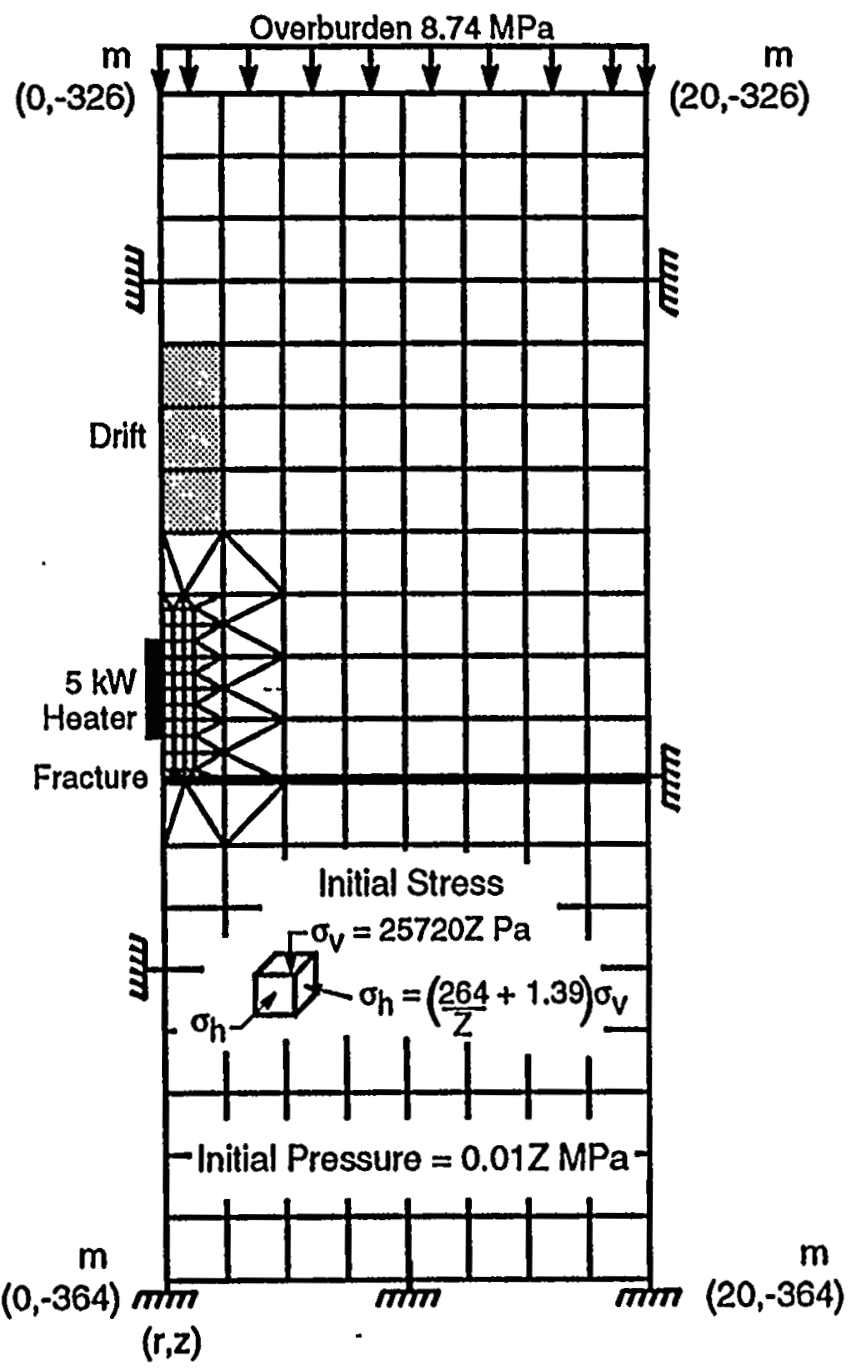

(a)

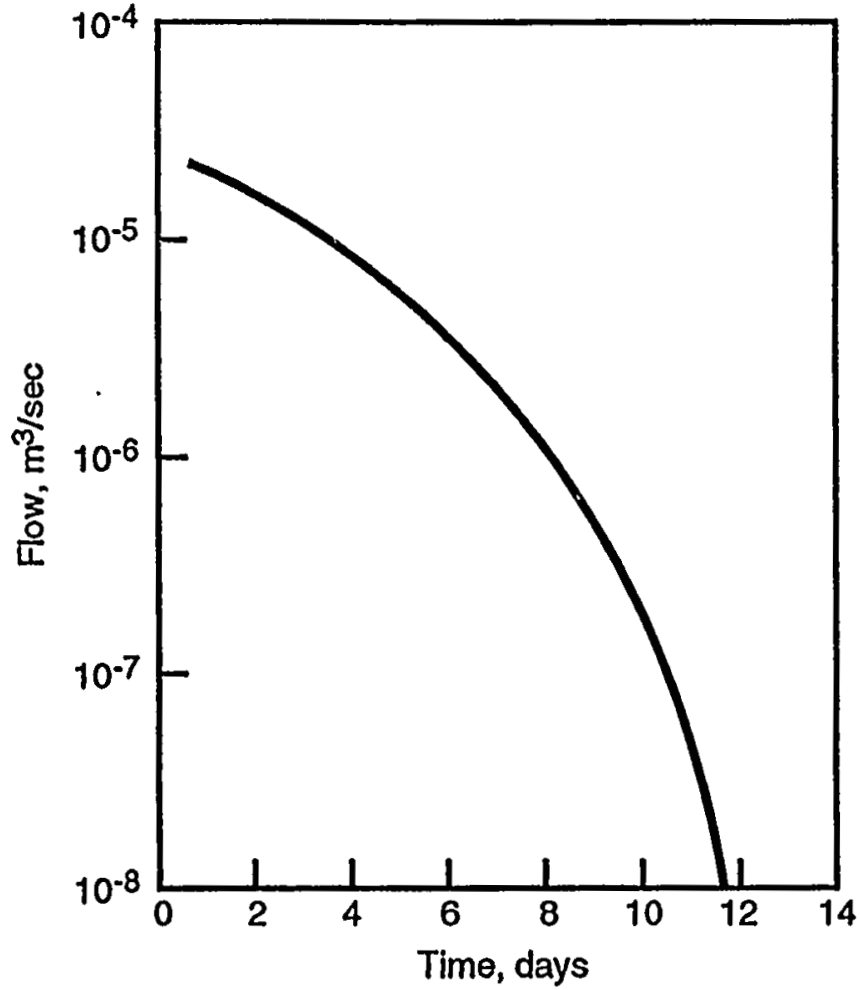

(b)

Figure 3-1. Coupled THM analyses using ROCMAS showing (a) finite element model of the source environment and (b) variation of fluid inflow to the heater borehole as a function of time after heater is turned on (Noorishad et al., 1984). 


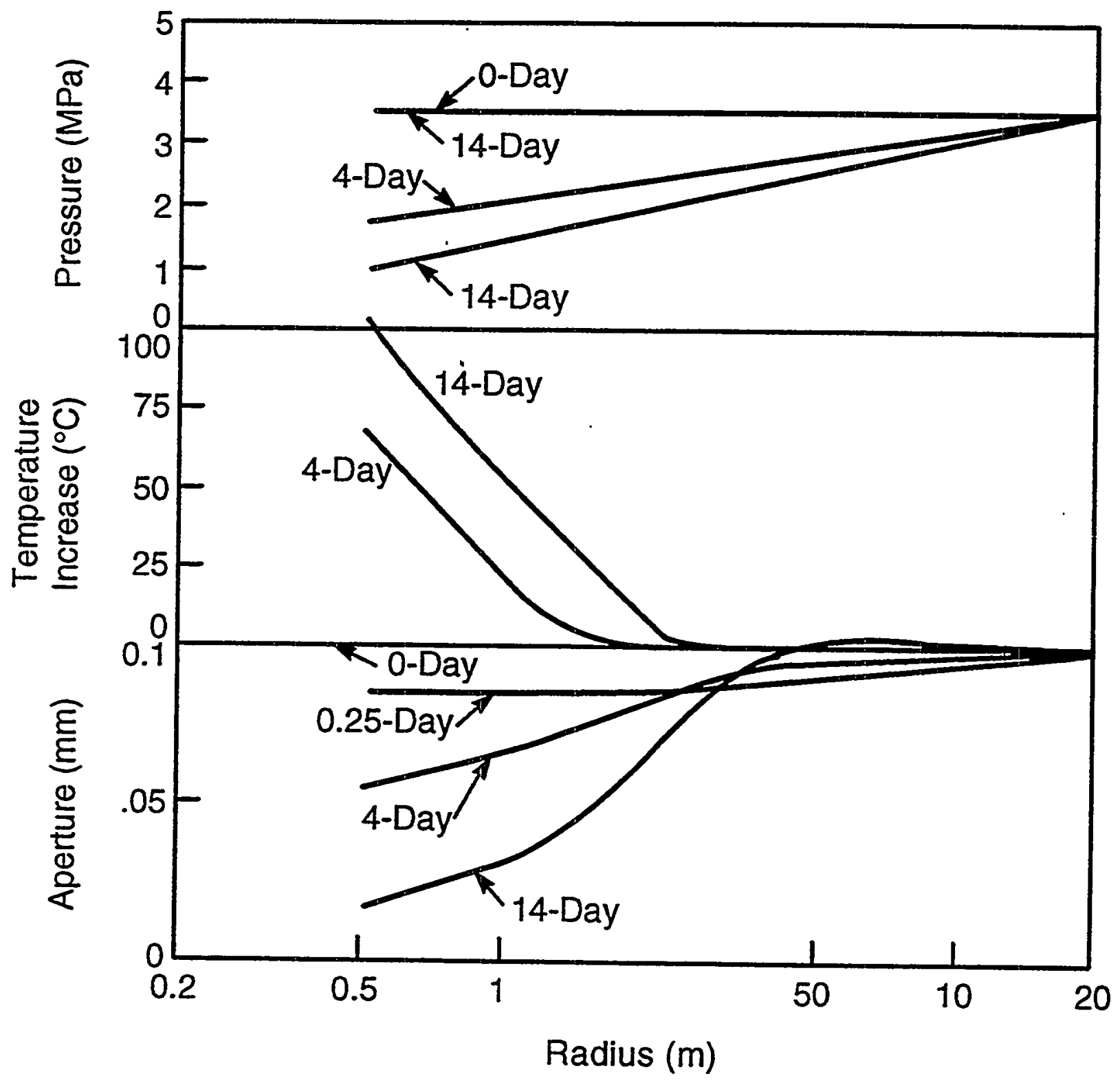

Figure 3-2. Pressure and aperture profiles in the fracture generated by ROCMAS for various durations and temperature profiles along heater midplane (Noorishad et al., 1984). 


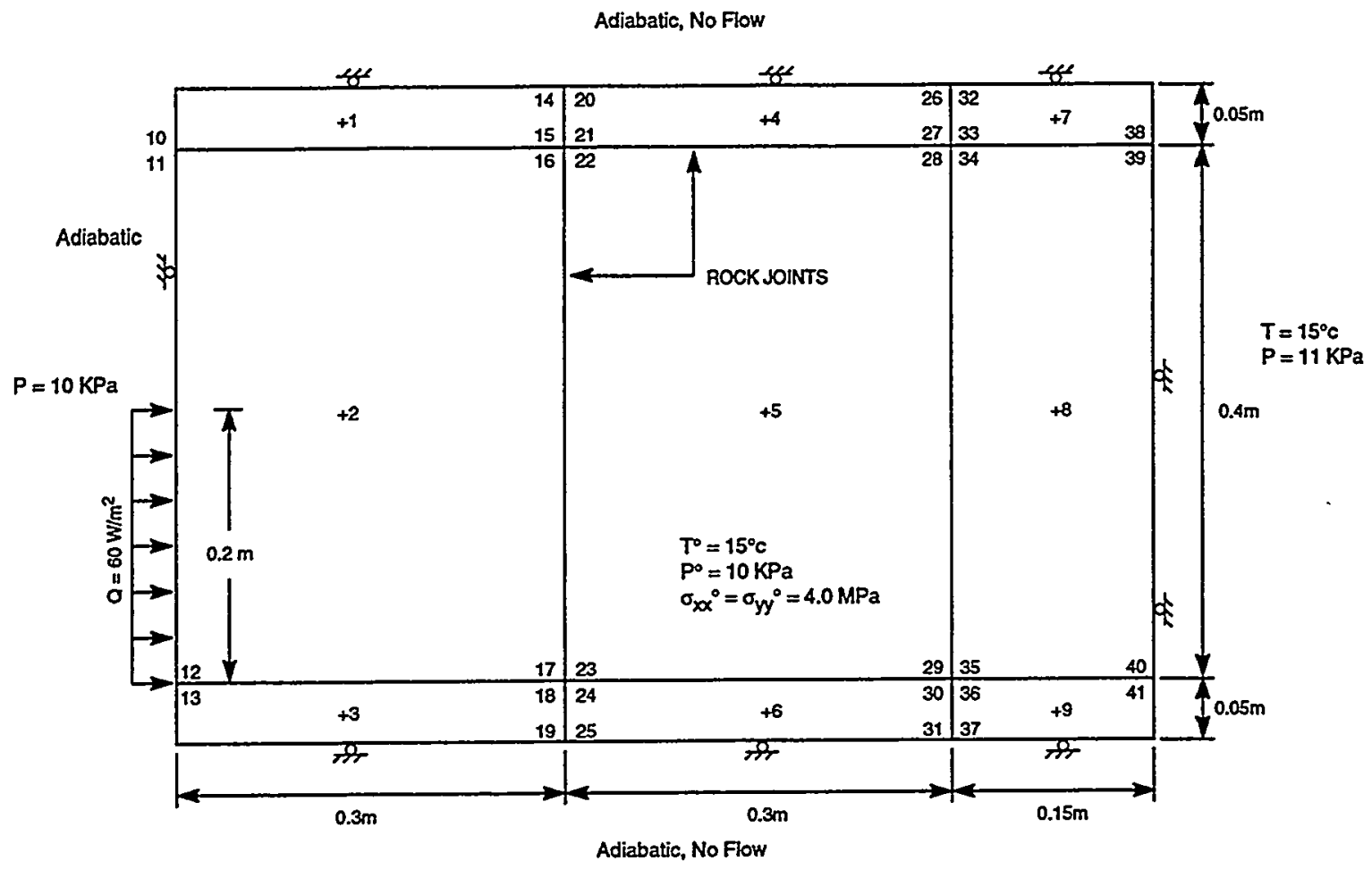

(a)

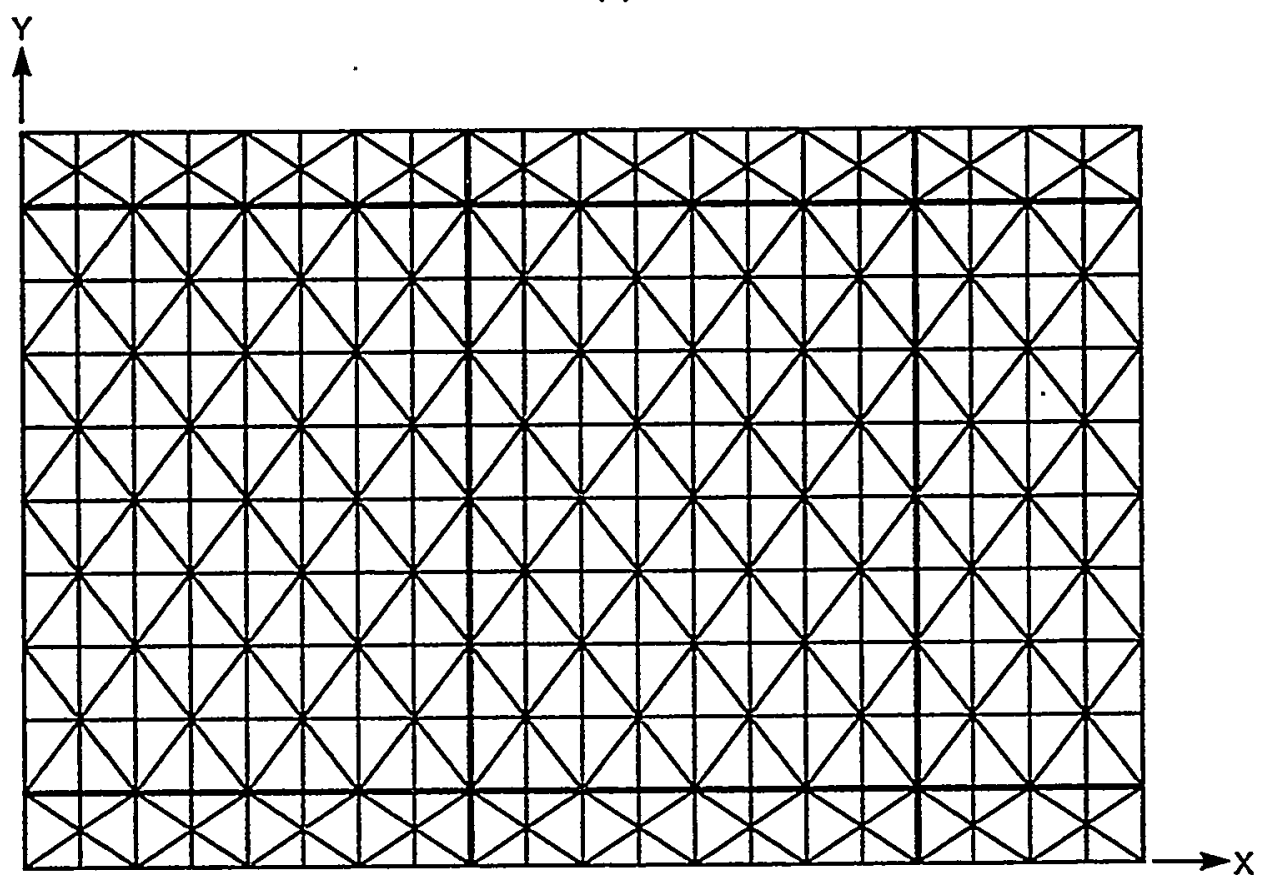

(b)

Figure 3-3. Coupled thermal-hydrologic-mechanical (THM) benchmark problem for DECOVALEX. 
For the thermal analyses, an initial temperature distribution of $15^{\circ} \mathrm{C}$ was assumed. A constant flux of 60 $\mathrm{W} / \mathrm{m}^{2}$ was applied to the lower left model boundary over a length of $0.2 \mathrm{~m}$ as shown in Figure 3-3(a). All other portions of the model boundary were assumed to be adiabatic.

Figure 3-3(b) shows the blocks discretized into finite difference triangles for use by the computer code UDEC for this benchmark problem. Since UDEC is based on the discrete element method, the fractures as shown in Figure 3-3(a) are explicitly incorporated in the analysis. Thus, the blocks are allowed to slide relative to one another, rotate, as well as deform elastically. Figure 3-4(a) shows the temperature contours obtained from UDEC after steady-state conditions have been achieved. For this particular problem, the specified heat flux resulted in a maximum temperature rise of approximately $6.2{ }^{\circ} \mathrm{C}$, as seen along the left edge of the model as shown in Figure 3-4(a) (Ahola et al., 1992). The thermal expansion accompanying this temperature rise resulted in a slight increase in the mechanical stress state, as well as a decrease in apertures up to 18 microns along portions of the vertical and horizontal joints. Even so, fluid flow still remained primarily in the horizontal fractures as a result of the large initial hydraulic aperture and negligible buoyancy effects. The UDEC code does not have the capability to model flow within the matrix, nor thermal-hydrologic coupling. Thus, the impact of forced convection within the fractures was neglected in Figure 3-4(a). Based on engineering estimates of the effect of forced convection within the fractures, a case was run in which the gridpoints along horizontal fractures were fixed at $15{ }^{\circ} \mathrm{C}$. Figure 3-4(b) shows the resulting temperature contours which represent more closely the true solution with only a maximum temperature rise of approximately $2{ }^{\circ} \mathrm{C}$. For this particular problem, it was found that the effect of the $\mathrm{H} \Rightarrow \mathrm{T}$ coupling was of importance mainly due to the large aperture and flow rates. Had the aperture been smaller or the rock matrix and fractures partially saturated as at Yucca Mountain, the hydrologic impact on the temperature field would have been negligible. A maximum temperature rise of approximately $2{ }^{\circ} \mathrm{C}$ was also obtained with the finite-element computer code MOTIF (Jing et al., 1993). The MOTIF code simulated flow within the fractures and matrix as well as conductive and convective heat transfer.

\subsubsection{Computer Codes for THM Modeling}

\subsubsection{ROCMAS}

ROCMAS (ROC Mass Analysis Scheme) is a finite element code which is used to investigate coupled THM behavior of liquid-saturated, fractured porous rocks (Noorishad et al., 1984; Noorishad and Tsang, 1989). The variational method is used to formulate the hydromechanical part of the THM phenomena. A Galerkin method is used to obtain a numerical formulation for the energy equation. Two different schemes of time integration are used to integrate the final matrix equations. A predictor-corrector scheme is used for the integration of the implicitly-coupled hydromechanical equations. For the energy equation, the Crank-Nicolson step-by-step procedure is used. The solution technique for both symmetric and nonsymmetric matrices, finalized in the above procedures, is the LU decomposition Gaussian Method. A tri-linear law defines the closure behavior of the fractures and an ideal elastic-plastic law models its shear behavior. Peak shear strength is obtained from a Coulomb criterion.

\subsubsection{GENASYS}

GENASYS (Geotechnical ENgineering Analysis SYStem) is a 2D and 3D hybrid boundaryelement finite-element code for computing the coupled fracture flow, heat flow, and deformation response of fractured rock masses (Wijesinghe, 1989). In GENASYS, the fractures in the rock mass are represented 


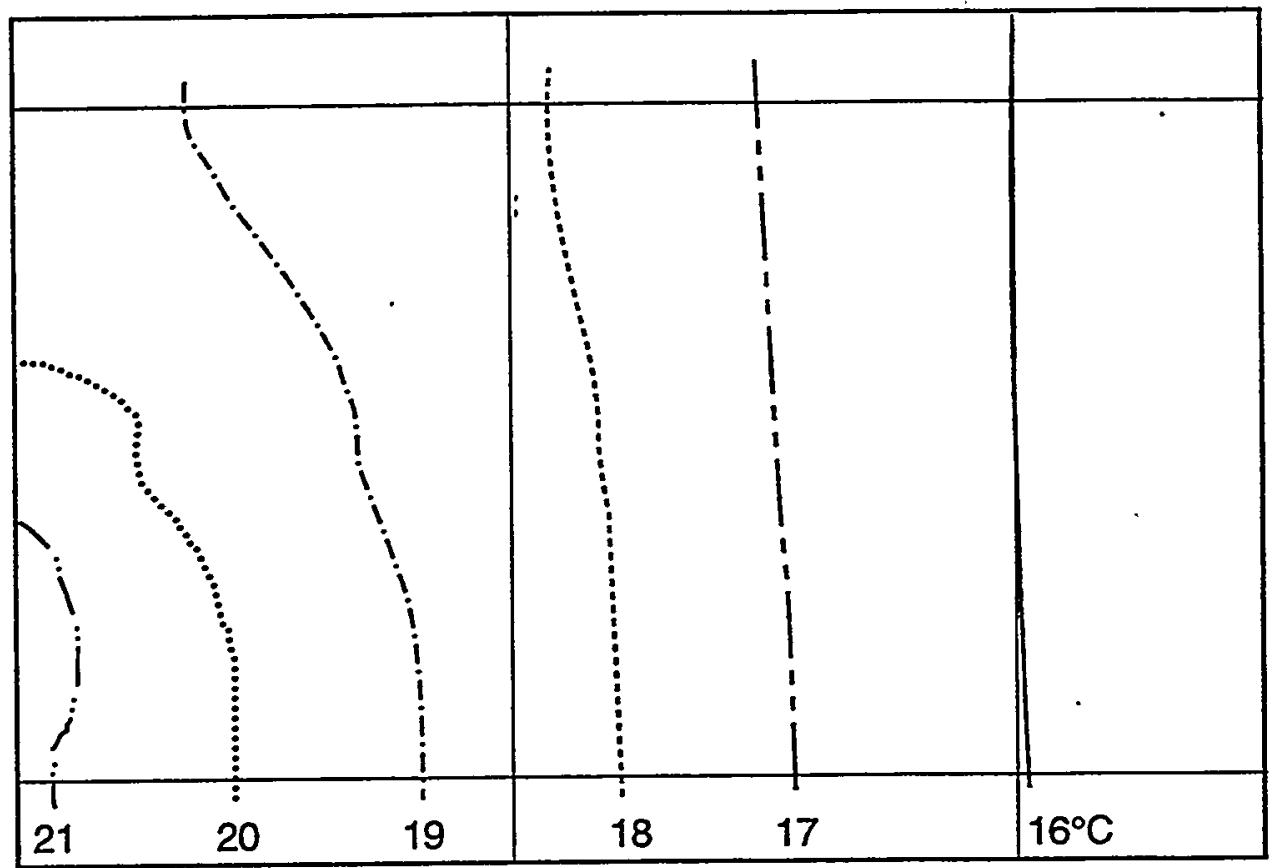

(a)

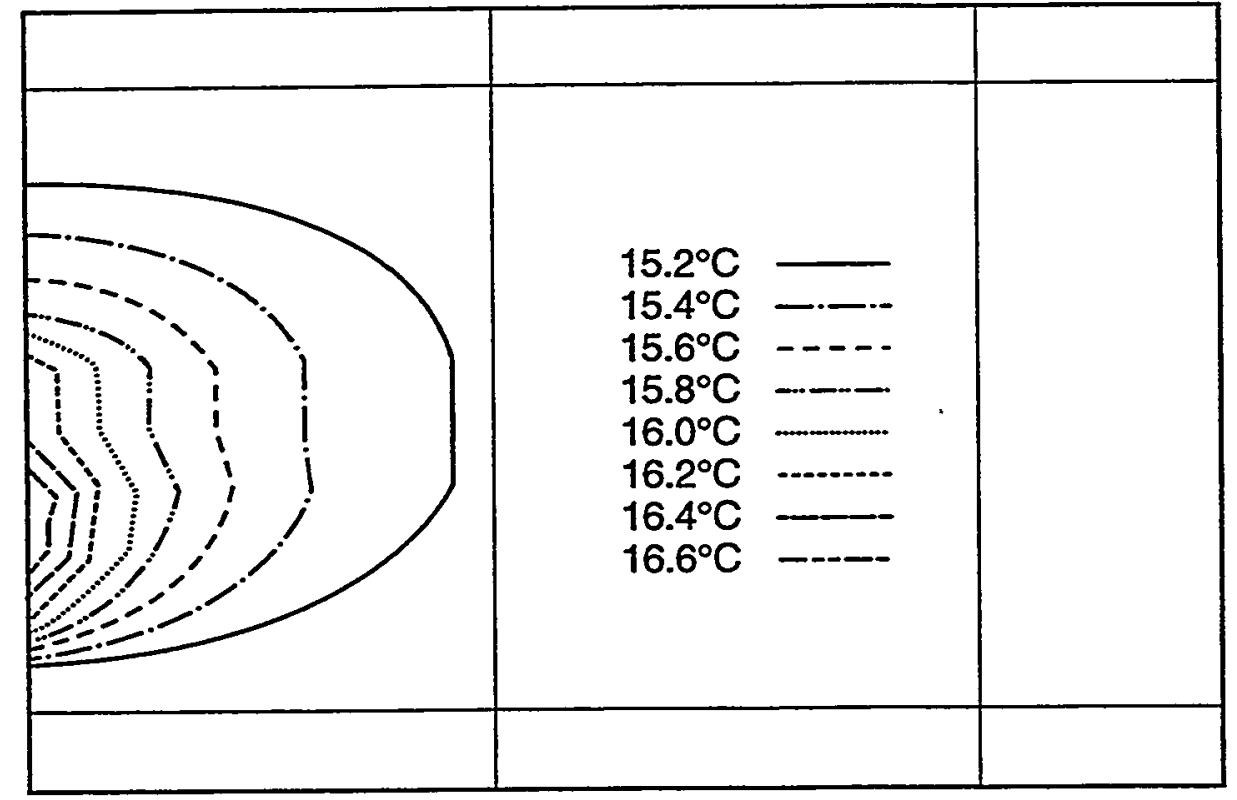

(b)

Figure 3-4. Coupled THM benchmark problem for DECOVALEX. (a) Temperature contours assuming only conductive heat transfer through the rock medium. (b) Temperature contours taking into account conduction, as well as the effect of forced convection through the horizontal fractures. 
as one or more discrete curved surfaces. The fracture surfaces can have any arbitrary shape and orientation, and can intersect to form a fracture network. The far-field response is treated primarily linearly elastic and nonlinearities are concentrated along fracture surfaces. The hybrid boundary-element finiteelement approach keeps the computational problem at the manageable level.

\subsubsection{THAMES}

THAMES 2D and 3D (Thermal, Hydraulic And MEchanical System analysis), (Ohnishi et al., 1990 ) is a 3D finite-element code developed to perform analysis of coupled thermal, hydraulic, and mechanical behavior of saturated and partially-saturated geologic media. The mathematical formulation of the model utilizes the Biot's theory of consolidation, with the Duhamel-Neuman form of Hooke's law and the energy balance equation. Coupled thermal, hydraulic, and mechanical processes are analyzed, and the coupled equations are solved simultaneously. Nonlinear heat conductivity, specific heat, and thermal expansion may be used in the computation. Stress-dependent fracture permeability of rocks, and permeability as a function of void ratio of soils, may also be incorporated into the analysis. THAMES 3D models an elastic medium and is unable to simulate phase change of water. A ID consolidation test has been used to verify the hydromechanical behavior of the code. Thermal stresses induced in a thick-walled cylinder with a constant temperature gradient have been used to test the mechanical-thermal behavior. Heat transfer in moving groundwater has been the third problem for THAMES 3D verification. This is the only method for verification since coupled THM processes are not solved analytically at present. Calculations of a synthetic, deep, nuclear-waste repository have been performed using the $2 \mathrm{D}$ and $3 \mathrm{D}$ versions of THAMES (Ohnishi et al., 1990).

\subsubsection{FEHMS}

FEHMS (Finite Element Heat Mass Stress) was developed for solving coupled, and nonlinear, fluid flow-heat transfer-solid deformation problems in porous, fractured, geological media (Kelkar and Zyvoloski, 1991). The code uses an implicit time-differencing scheme, which allows for larger time steps several orders of magnitude larger than those expected for explicit schemes. The code is capable of 3D simulations.

\subsubsection{ABAQUS}

ABAQUS (ABAQUS, 1988) is a finite-element computer program for numerical modeling of structural response. It can be used for both $2 D$ and $3 D$ structural modeling. A large class of stress analysis problems can be solved with ABAQUS, for both static and dynamic response. For static stress analysis, both linear static and nonlinear static analysis can be conducted. Geometrically nonlinear static problems involving buckling or collapse behavior can also be solved. Time-dependent material response in static analysis may involve creep or swelling (generally occurring over long periods of time), or rate-dependent yield (which is often important in fairly rapid processes, such as metal working problems). Conductive heat-transfer (diffusion) problems can be solved with ABAQUS. The problems can be transient or steadystate, linear or nonlinear. ABAQUS also contains a procedure for performing two-way coupled heattransfer/stress analysis. ABAQUS provides the capability for removing elements from the model or adding elements to the model. Such capabilities could be used for simulating excavation and support sequences for analyzing problems in geomechanics. In addition, ABAQUS provides a capability for modeling coupled pore fluid diffusion/stress analysis problems. 


\subsubsection{SANGRE}

SANGRE (Anderson, 1986) is a 2D finite-element program with coupled fluid flow and structural deformation capabilities including large deformation and faulting, as well as heat transport by conduction and convection at each time step. Temperature changes occur through heat changes in the medium, as well as through conduction in the solid and convection of heat by the interstitial fluid. Fault planes in the geologic medium are modeled as slide lines. Large-scale slip across the fault planes can be modeled using these slide lines. The rezone capability of SANGRE can be used to solve problems where severe mesh deformation can occur. This program was developed by LANL in cooperation with the Geological Research Division of ARCO.

\subsubsection{UDEC}

UDEC (Board, 1989b) is a 2D discrete-element code with mechanical, thermal, and hydrological capabilities to model the behavior of the jointed rock mass. It belongs to the subclass distinct element method as it uses an explicit time-marching scheme to solve the equations of motion directly. The bodies can be either rigid or deformable, while contacts are deformable. The heat transfer is based on conductive transfer within the medium with the provision for temperature, flux, convective, or radiative boundaries. Only one-way coupling from thermal to mechanical is considered. It also has the capability to adopt an implicit finite-difference scheme. Only fluid flow through the joints is considered, and the analysis is coupled with the mechanical-thermal analysis. Fluid flow through the rock matrix is not modeled. The approach used in performing a coupled analysis is to first run the problem out to some specified number of thermal time steps. At this point, the thermal or real time is held constant while mechanical cycling is performed to reach a new equilibrium state. This process is then continued. The requirement in performing the simulation in this manner is that temperature increases between successive thermal time steps cause only "small" out-of-balance forces in blocks, especially for nonlinear problems.

\subsubsection{Summary and Conclusions}

In this section, the three-way coupling between thermal, hydrologic, and mechanical processes has been discussed. Much of the literature related to these three processes has been primarily numerical studies, with the computer codes being verified against available analytic solutions for the various possible two-way couplings. Due to the lack of analytic solutions for three-way couplings, laboratory and field studies are recommended for validating computer codes. Cooperative international projects, such as DECOVALEX, are important in providing the necessary database of experimental studies.

\subsection{THERMAL-HYDROLOGIC-CHEMICAL COUPLING}

As discussed in Section 2, each of the four processes being considered (thermal, hydrologic, mechanical, and chemical), are involved in a series of two-way couplings. The relative importance of each of these couplings varies, as does the degree to which the effects involved are understood.

Many of the equations necessary for the modeling of the various two-way couplings have been presented in Section 2. However, three-way coupling between these processes is much less wellunderstood. As a result, there is relatively little available in the way of computer codes for modeling the interaction between more than two processes. With respect to nonlinear chemical processes, an iterative approach to coupling offers the best chance of success for numerical predictions in the proposed repository 
system (Yeh and Tripathi, 1989). The discussion presented in this section is limited to presenting phenomena either postulated or observed. The occurrence of many of these in a natural environment awaits confirmation in many cases.

In a discussion of the effect of coupled processes on solute transport in the near-field, Jamet et al. (1990) characterized coupling as being of two types. The first type involves those phenomena that result from the effects of coupling on material properties for the solid, fluid, and gas phases (e.g., density, viscosity, vapor pressure). This can also include those couplings that can affect properties of the medium of interest (e.g., permeability, porosity), such as mineral dissolution/precipitation in fractures and pores which is also discussed in Sections 2.10.1 and 2.10.2. The second type includes fluxes in fluid, heat, and solute that appear in response to gradients in temperature, fluid pressure, and solute concentration. These have been referred to as constitutively-coupled processes (Carnahan, 1984a,b; 1987a). While constitutivelycoupled processes include many possible effects such as thermal filtration and thermal osmosis, Jamet et al. (1990) indicated that only a few have a significant effect on solute transport. Those that involve coupling between thermal, hydrologic, and chemical processes are discussed in this section.

\subsubsection{Effects of Coupling on Material Properties and Media Characteristics}

The porosity and permeability of a given medium may be enhanced by the dissolution of the minerals that make up the matrix of the medium. An extreme example of this type of porosity enhancement is the dissolution of limestones in karst terrain to produce sinkholes and caves. Conversely, precipitation may serve to plug available porosity, reducing permeability (Section 2.10) (Steefel and Lasaga, 1990). As discussed in Section 2.5, mineral solubility is strongly dependent on the pressure and temperature of the system of interest. Many common minerals such as quartz, fluorite, anhydrite, and metal sulfides exhibit a prograde solubility, such that precipitation (i.e., decreasing solubility) is favored with decreasing temperature (Holland and Malinin, 1979; Cathles, 1983). Silica scale in geothermal wells is due in large part to the cooling and/or boiling of silica supersaturated fluids as they rise to the surface (Cline et al., 1992). In contrast, at a given constant $\mathrm{pH}$ and $\mathrm{p}\left(\mathrm{CO}_{2}\right)$, carbonates such as calcite and dolomite tend to precipitate from solution with increasing temperature. This is known as retrograde solubility, and is in evidence as the carbonate scaling in hot-water heaters. Increased temperature may alter primary minerals to clays and other secondary minerals, plugging porosity and reducing formation permeability (Nagy et al., 1990). Precipitation and dissolution may also be controlled by kinetic processes. For example, while calcite precipitates readily, silica precipitation is kinetically controlled in geothermal systems and may not occur for some time after saturation has been reached (Cathles, 1983; Thomas and Gudmundsson, 1989).

The effect of temperature is only one control on mineral solubility; other critical parameters include solution $\mathrm{pH}, \mathrm{p}\left(\mathrm{CO}_{2}\right), \mathrm{p}\left(\mathrm{O}_{2}\right)$, and salinity (Thomas and Gudmundsson, 1989; Murphy, 1990). For example, boiling of a solution through pressure release will preferentially fractionate dissolved $\mathrm{CO}_{2}$ from the liquid $\left[\mathrm{CO}_{2(\mathrm{aq})}\right]$ to the vapor phase $\left[\mathrm{CO}_{2(\mathrm{~g})}\right]$, and calcite will precipitate (Thomas and Gudmundsson, 1989). The amount of calcite deposited will vary depending on the composition and temperature of the solution. More calcite will be deposited in response to $\mathrm{CO}_{2(\mathrm{~g})}$ release at lower temperature. In addition, more calcite will precipitate with increasing ionic strength (Holland and Malinin, 1979). In geothermal systems, boiling can also oxidize sulfide minerals and $\mathrm{H}_{2} \mathrm{~S}$ in solution, leading to the formation of acidsulfate waters. Waters under these conditions exhibit low pH $(<1$ to 3$)$, which leads to the hydrolysis and dissolution of common rock-forming minerals such as feldspars, and the formation of clay minerals such as kaolinite (Rose and Burt, 1979; Henley et al., 1985). Boiling of the fluid associated with the 
emplacement of $\mathrm{HLW}$ can result in fractionation of $\mathrm{CO}_{2}$ to the gas phase, leading to significant variations in $\mathrm{pH}$ for the coexisting groundwater, affecting aqueous speciation and mineral solubility (Murphy, 1990).

Given the temperature dependent solubility of different minerals, it is possible that solutions (both liquid and gas phase) moving by thermally-driven convection will redistribute chemical components such as silica and calcite. Increases in temperature also lead to increases in molecular diffusion coefficients. Oelkers and Helgeson (1988) reported increases of as much as two orders of magnitude from $25^{\circ} \mathrm{C}$ to $300^{\circ} \mathrm{C}$. This can lead to enhanced transport even in the absence of significant convection. This type of redistribution has been observed in laboratory heater experiments with Yucca Mountain tuff under partially-saturated conditions (Rimstidt et al., 1989). Silica and iron dissolved near the heater, were transported in solution and precipitated as amorphous silica, iron hydroxides, clay, and zeolite at the cooled end of the system. In the nonisothermal transient experiments of Lin and Daily (1990) on samples of the Topopah Spring tuff, permeability was progressively reduced by three orders of magnitude from $1.3 \times 10^{-14} \mathrm{~m}^{2}$ to about $10^{-17} \mathrm{~m}^{2}$ due to narrowing of fracture aperture by silica deposition. Experiments of Vaughan (1987) using granite cores indicated that although porosity was reduced by a relatively small amount, permeability was reduced by over 95 percent.

Numerical simulations have also been used to predict the redistribution of silica and calcite. Using a modified version of the TRACR3D code (Section 2.9), Travis and Nuttall (1987) suggested that reduced permeability due to quartz reprecipitation may enhance waste isolation. In contrast, Verma and Pruess (1988) used the code MULKOM (Pruess, 1983) to model equilibrium silica precipitation and dissolution, and determined that the amount of silica redistribution in a hydrologically saturated fractured medium did not have a significant effect on near-field temperatures, pore pressures or fluid flow. Simulations of ${ }^{14} \mathrm{C}$ transport in partially-saturated rock (Codell and Murphy, 1992) indicate that after an early initial release of ${ }^{14} \mathrm{C}$ to the gas phase, calcite will precipitate and will serve to sequester ${ }^{14} \mathrm{C}$ at longer times.

Mineral precipitation and dissolution can also affect the retardation of radionuclide migration, due to introduction and/or removal of sorptive minerals (Turner, 1991a). Minerals such as zeolites, clays, and oxides can be dissolved and reprecipitated, depending on temperature and fluid chemistry. In addition, removal of radionuclides from solution, either due to the precipitation of stoichiometric radioelement compounds or coprecipitation as an impurity in other minerals, is also affected by the temperature (and chemistry) of the solution. By machining circular flow channels into granite blocks, Walton et al. (1985) constructed thermal-convection loops to study the effects of heat and mass transport on radionuclide migration. A $40{ }^{\circ} \mathrm{C}$ temperature difference was applied across the system. Several radionuclides $\left({ }^{125} \mathrm{Sb}\right.$, ${ }^{60} \mathrm{Co}$, and ${ }^{54} \mathrm{Mn}$ ) were concentrated at the hot side of the experiment, probably by sorption on iron oxyhydroxides. ${ }^{144} \mathrm{Ce}$ and ${ }^{99} \mathrm{Tc}$ were present in elevated concentrations on the cold side of the apparatus.

Because many common minerals exhibit prograde solubility, higher temperatures, particularly in the near-field environment, allow kinetic barriers to be overcome and result in a greater ionic strength for the groundwater. This tends to collapse the electrostatic double layer formed around electricallycharged particles such as colloids (McDowell-Boyer et al., 1986). This reduces electrostatic repulsion, and promotes particle attachment and aggregation. This, in turn, will reduce colloid transport of radionuclides. 


\subsubsection{Constitutive Couplings}

Camahan (1984b) and Jamet et al. (1990) discuss thermodynamically-coupled constitutive equations (e.g., thermal osmosis, chemical osmosis, the Soret effect). For fluid, heat, and species fluxes, the possible constitutive couplings are summarized in Table 3-1. With regard to coupling thermal, hydrologic, and chemical processes, these can be divided into two major categories: transport associated with volume flux (advection) and transport relative to fluid motion (diffusion). Transport associated with volume flux is divided into three fluxes: thermal osmosis (temperature gradient), advection (pressure gradient), and chemical osmosis (chemical gradient). Three diffusional fluxes include thermal (or Soret) diffusion in response to the temperature gradient, ultrafiltration (or reverse osmosis) in response to pressure gradients, and mass diffusion according to Fick's Law in response to concentration gradients. The primary couplings are Fourier's, Darcy's, and Fick's laws; however, secondary couplings may need to be considered in special cases.

Table 3-1. Summary of driving potentials, fluid pressure, temperature, and species concentration, and how they are expected to generate a flux of fluid, heat, and/or species.

\begin{tabular}{|c|c|c|c||}
\cline { 2 - 4 } \multicolumn{1}{c|}{} & \multicolumn{3}{|c|}{ Driving Potential } \\
\hline \hline Flux & $\begin{array}{c}\text { Fluid Pressure } \\
\text { Gradient }\end{array}$ & $\begin{array}{c}\text { Temperature } \\
\text { Gradient }\end{array}$ & $\begin{array}{c}\text { Species } \\
\text { Concentration } \\
\text { Gradient }\end{array}$ \\
\hline \hline Fluid & Darcy's Law & Thermal Osmosis & Chemical Osmosis \\
\hline Heat & Thermal Filtration & Fourier's Law & DuFour Effect \\
\hline Species & $\begin{array}{c}\text { Reverse Osmosis or } \\
\text { Ultrafiltration }\end{array}$ & Soret Effect & Fick's Law \\
\hline
\end{tabular}

In 1D simulations of transport, a single ideal solute across a $1-\mathrm{m}$ column of saturated clay, Carnahan (1984a) has reported that, when processes are coupled, the water flux due to thermal osmosis can exceed the Darcy flux by as much as three orders of magnitude, when subjected to a temperature gradient of $2{ }^{\circ} \mathrm{C} / \mathrm{m}$. For compacted clays and a temperature gradient of about $20^{\circ} \mathrm{C} / \mathrm{m}$, water flux due to thermal osmosis can reach $10^{-8} \mathrm{~m} / \mathrm{s}$ (Jamet, et al., 1990).

The equations describing advection, the movement of fluid in response to pressure gradients, have been presented in Appendix B. Chemical osmosis, the movement of water in response to concentration gradients, has also been suggested by Carnahan (1984a) to be a major factor in fluid flux. Numerical simulations indicate that water flux in clays from less to more concentrated zones can be as much as 3 to 4 orders of magnitude greater than Darcy's flux. This is counterbalanced by the flux of solute back across the membrane by pressure gradients (ultrafiltration/reverse osmosis). In coupled systems, Carnahan indicates that reverse osmosis is of minor significance compared to chemical osmosis.

Once the fluid has moved in response to the system gradients, solute can also move in and out of the fluid parcel. Thermal or Soret diffusion involves the movement of solute in response to the temperature gradient. In an isothermal, isobaric system (solution, solid, or gas) initially at chemical equilibrium, the imposition of a thermal gradient leads to a redistribution of chemical species as the 
solution seeks to reattain chemical equilibrium. These processes may become more important for increasing temperature gradients (Jamet et al., 1990; Guy and Schott, 1992). Jamet et al. (1990) note that Soret diffusion can act as an ore deposition mechanism in geothermal environments. The effect tends to be more pronounced for lighter elements; less volatile species tend to concentrate near the cold parts of the system. The sensitivity of a given element to Soret diffusion is measured by the Soret coefficient, $S_{\mathrm{T}}$ :

$$
S_{T}=\frac{D^{\prime}}{D_{m}}=\frac{\nabla f_{I}}{f_{I}\left(1-f_{I}\right) \nabla T}
$$

where

$$
\begin{array}{ll}
\mathrm{S}_{\mathrm{T}} & =\text { Soret coefficient }\left(\mathrm{K}^{-1}\right) \\
\mathrm{D}^{\prime} & =\text { thermal diffusion coefficient }\left[\mathrm{m}^{2} /(\mathrm{s}-\mathrm{K})\right], \\
\mathrm{D}_{\mathrm{m}} & =\text { molecular diffusion coefficient }\left(\mathrm{m}^{2} / \mathrm{s}\right), \\
\mathrm{f}_{\mathrm{I}} & =\text { mass fraction of solute } \mathrm{I} \text { (dimensionless), and } \\
\mathrm{T} & =\text { temperature }(\mathrm{K}) .
\end{array}
$$

$S_{T}$ values are generally positive, as opposed to the temperature gradient. Values range from about $10^{-2}$ to $10^{-3} \mathrm{~K}^{-1}$ for glasses and aqueous solutions (Jamet et al., 1990; Guy and Schott, 1992). Studying Soret element migration in radioactive glassforms, Guy and Schott (1992) report that relatively-light element compounds such as $\mathrm{Na}_{2} \mathrm{O}, \mathrm{B}_{2} \mathrm{O}_{3}, \mathrm{Li}_{2} \mathrm{O}$, and $\mathrm{H}_{2} \mathrm{O}$ are susceptible to migration by Soret diffusion, while predicting weak diffusion for heavier radioelements such as $\mathrm{Sr}, \mathrm{Cs}, \mathrm{Pu}, \mathrm{Am}$, and $\mathrm{Np}$. Thermal diffusion is relatively minor in the simulations of Camahan (1984a). Molecular diffusion according to Fick's Law in response to chemical gradients tends to balance the effects of Soret diffusion.

In fluids (liquid or gas), an additional coupling is found between the Soret effect and thermallydriven water convection and is called the thermogravitational effect (TGE) (Jamet et al., 1990; Guy and Schott, 1992). If the velocity due to convection is large, the fluid will tend to homogenize, and concentration gradients are diminished. If velocity is small, the coupling will be weak and the concentration gradient will be dominated by Soret diffusion.

In the literature reviewed, the importance of the secondary couplings appears to be negligible or uncertain in most cases of practical interest. Both Carnahan (1984a) and Jamet et al. (1990) present cases where the secondary couplings are non-negligible in clays. However, there is very little clay in the Topopah Spring tuff and there is currently little interest in clay backfills; hence, these examples may not be applicable to the present problem.

\subsubsection{Computer Codes}

Because of the inherent complexities, there are few codes at present that attempt to simulate the effects of the coupling between these three processes. As mentioned previously, most of the thermalchemical codes do not consider fluid flow. Coupled hydrogeochemical codes are limited to isothermal conditions, and most of the thermal-fluid flow codes do not explicitly consider chemistry. The codes discussed below (and also in Section 2.9) can handle variable temperature. Generally, however, the temperature gradient is specified for the problem; heat transfer is decoupled from fluid flow and chemistry, and its effects are not considered explicitly. 


\subsubsection{CHMTRNS}

As discussed in Section 2.9, CHMTRNS (Noorishad et al., 1987) is a one-step coupled program that has been used to model ${ }^{14} \mathrm{C}$ age-dating and silica reaction kinetics. The code is able to consider nonisothermal conditions. There is no accompanying thermodynamic database, and the data must be input specifically for each problem. The code has been limited to $1 \mathrm{D}$ systems. Because of the less efficient, direct coupling used by CHMTRNS, future application of the code to more complex problems over long time will probably be limited due to excessive computer requirements (Yeh and Tripathi, 1989).

\subsubsection{THCC}

THCC (Thermo-Hydro- ( 'zemical Coupling), begun as an extension of the CHEMTRN code (see Section 2.9), was developed at LBL (Carnahan, 1987b,c, 1988, 1990) to model isothermal and nonisothermal hydrochemical transport. The code can model both homogeneous and heterogeneous chemical reactions of aqueous speciation, precipitation/dissolution, redox, and ionic strength/activity effects. Ion exchange can also be modeled, to a limited extent. Temperature-dependent chemistry is modeled by using equilibrium constant data fit to a $\log (\mathrm{K})$-temperature relationship similar (but not identical to) the form presented in Eq. 2-17. For a constant flow velocity under hydrologically saturated conditions, chemistry and hydrology equations are directly coupled (one-step). Heat transfer, however, is decoupled and solved iteratively. It is assumed that heat transport has no effect on fluid flow, and that the heats of reaction do not affect the temperature distribution. The necessary equilibrium constant data and the constants for the log $(\mathrm{K})$-temperature relationship are provided as input for each run. It is currently limited to ID simulations, or radial flow, and has been used to model uranium oxidation/reduction and migration in a simplified $\mathrm{Si}-\mathrm{Ca}-\mathrm{U}-\mathrm{CO}_{2}-\mathrm{H}_{2} \mathrm{O}$ environment.

\subsubsection{Codell and Murphy (1992)}

For a limited representation of the $\mathrm{Ca}-\mathrm{CO}_{2}-\mathrm{H}_{2} \mathrm{O}$ system, Codell and Murphy (1992) have iteratively coupled nonisothermal equilibrium chemistry with the gas flow code described by Amter and Ross (1990) to model ${ }^{14} \mathrm{C}$ transport and retardation through partially-saturated rock. Applied specifically to Yucca Mountain, a vertical 1D column was constructed with dimensions and boundary conditions appropriate to the proposed repository. Liquid saturation was approximated with an empirical model, based on numerical simulations of Nitao (1988), with a maximum saturation of 80 percent. Gas was assumed to migrate upwards only and ${ }^{14} \mathrm{C}$ transport was assumed only to occur by advection in the gas phase. ${ }^{14} \mathrm{C}$ is removed from solution by calcite precipitation and by radioactive decay. Gas flow into and out of each cell is calculated for a given time-step by the transport model. Mass distributions between the gas, liquid, and solid phase are then calculated by a simplified geochemical model, and updated for the subsequent time-step. Within the constraints of the assumptions made, the model results indicate that the aqueous solution remains the largest reservoir of ${ }^{14} \mathrm{C}$. Immobilization in the aqueous phase results in a retardation by a factor of 30 to 40 relative to gas transport. Calcite precipitation also acts as a significant long-term sink for ${ }^{14} \mathrm{C}$.

\subsubsection{HYDROGEOCHEM}

As discussed in Section 2.9, HYDROGEOCHEM (Yeh and Tripathi, 1990, 1991) was developed at ORNL. The code is very elaborate and able to accommodate a wide variety of conditions and processes. The code is able to account for changes in chemical equilibrium due to the effects of temperature, pressure, and ionic strength. It does not appear at present, however, that the effects of heat on fluid flow are explicitiy incorporated in the model. The code has been used to simulate several different types of problems in an effort to evaluate the effects of different processes on contaminant transport. 


\subsubsection{Cline et al. (1992)}

Cline et al. (1992) have modified the earlier 1D model of Donaldson (1968) to simulate twophase flow and quartz precipitation in geothermal systems. The conceptual model is one of a single-phase fluid entering the base of the system at a constant temperature and mass flux. Temperature is fixed at $100{ }^{\circ} \mathrm{C}$ and pressure is fixed at $1 \mathrm{bar}$ at the surface. As the fluid rises due to buoyancy effects, temperature decreases as a function of pressure decreases along the pure $\mathrm{H}_{2} \mathrm{O}$ liquid/vapor curve. If the mass flux of the fluid exceeds a threshold value of about $10^{-6} \mathrm{~kg} / \mathrm{m}^{2}-\mathrm{s}$, the saturation pressure fixed by the liquid/vapor curve will exceed the confining pressure of the system and the fluid will boil. Permeability is allowed to vary vertically, and relative permeability of liquid and vapor phases are modeled as a function of temperature for the one-component $\mathrm{H}_{2} \mathrm{O}$ system. Silica chemistry is controlled by fluid/quartz equilibria, and it is assumed that the fluid is saturated with quartz when it is input at the base of the system. Vein walls are assumed to be quartz, and the fluid maintains equilibrium with quartz as it rises to the surface. Quartz precipitates as the fluid cools and as a result of decreased solubility with the formation of a vapor phase. The effect of quartz precipitation on permeability is not specifically considered. Cline et al. (1992) examined variations in the height of the boiling column, amount of vapor generated, and the intensity of quartz precipitation for ranges in temperature, mass flux, and permeability reasonable for geothermal systems.

\subsubsection{MPATH}

As discussed in Section 2.9, MPATH (Lichtner, 1988, 1992) is able to model the migration of reaction fronts as a function of time and temperature using the quasi-stationary state approximation. Nonisothermal systems can be simulated by stipulating a temperature profile as a function of time and space. Lichtner (1992) imposed a time-invariant Gaussian temperature profile from $25^{\circ} \mathrm{C}$ to $300^{\circ} \mathrm{C}$ to simulate the hydrothermal alteration of a hypothetical igneous host rock at a $1 D$ kilometer scale for times up to 200,000 years. Potassium feldspar and quartz tended to dissolve upstream from the heat source and precipitate downstream as the fluid cooled. Albite (Na-plagioclase feldspar) exhibited the opposite behavior, precipitating upstream and dissolving downstream from the heat source. Eventually, quartz precipitating downstream from the heat source filled the porosity of the medium. The model is run for a constant velocity, and does not take into account the effects of heat on fluid properties (e.g., $\rho$ ) or thermally induced convection.

\subsubsection{Summary and Conclusions}

In this section, coupled THC processes were discussed. Two types of couplings were discussed: material property couplings and phenomenological couplings (constitutive couplings). Material properties and media characteristics can be influenced, especially by temperature-dependent chemical processes. Dissolution, alteration, and/or precipitation may be important in the near-field where temperature variations are largest. Constitutive couplings represent fluxes of either fluid, heat, or species due to gradients in either pressure, temperature, or species concentration. The primary couplings are known as Fourier's, Darcy's, and Fick's laws; however, secondary couplings (thermal osmosis, chemical osmosis, the DuFour effect, etc.) may need to be considered in special cases. The literature was reviewed and the importance of the secondary constitutive couplings appears to be either negligible or uncertain in cases of practical interest. Computer codes were also summarized where it was found that THC codes typically considered the temperature and fluid-flow fields as iterative input into geochemical codes. 


\section{FOUR-WAY COUPLED PROCESSES}

In this section, the four processes (thermal, hydrologic, mechanical, and chemical) are considered for the four-way coupled behavior. The individual one-way couplings were discussed in Section 2, and three-way couplings were discussed in Section 3. Hence, this section synthesizes and analyzes the previous discussions without introducing new coupling mechanisms. The purpose for studying a process is reviewed, and the importance of the couplings are compared so that the most important couplings can be identified.

\subsection{PERFORMANCE OBJECTIVES}

The performance objectives of a geologic repository dictate the type of studies that need to be performed. The processes of interest in this work are THMC coupled processes. The regulations of interest are summarized in 10 CFR Part 60, and especially in Sections 60.111,60.112, and 60.113 and the specific references to the "generally applicable environmental standard for radioactivity as may have been established by the EPA" [60.111(a) and 60.112]. The EPA standards stem from 40 CFR Part 191, which places limits on the cumulative release of radionuclides and sets limits on the annual dose to the public. Currently, the EPA standard is in remand and is expected to be revised and reissued. Regardless of precise definitions of repository performance, the general framework and general objectives of the regulations are considered clear. The repository is expected to be located, designed, constructed, operated, and eventually closed so that the workers and public are protected.

The performance objectives and related regulatory language suggest that the life of a repository can be discussed in three distinct time periods: operation, containment, and isolation [see 10 CFR 60.102(d) and (e)]. The operation period is the time until permanent closure, the containment period is from closure until 300 to $1,000 \mathrm{yrs}$, and the isolation period begins from the end of the containment period - the quantitative requirements for isolation being for 10,000 yrs. The performance objectives are distinct for each of the three time periods, and implicitly suggest which processes must be studied and sufficiently understood. For example, during the operation time period, mechanical processes and loads affect the stability of the underground excavations and may influence the waste retrievability option. Similarly, during the containment and isolation time periods, chemical processes control container corrosion, waste form dissolution, and radionuclide migration through the geologic setting.

The performance objectives for each time period can be discussed as requiring specific studies pertinent to specific processes. Hence, the performance objectives suggest which processes must be studied and sufficiently understood. The processes most closely related to the performance objectives depend on the time period of the repository. For example, during the operation time period, performance objectives are most closely related to mechanical processes, because mechanical processes and loads dictate the stability of underground excavations. Likewise, during the containment and isolation time periods, the performance objectives are most closely related to hydrological and chemical processes. A study of hydrological and chemical processes leads to an assessment of the corrosion of the waste containers, and the radionuclide migration through the geologic setting. Hence, an understanding of the repository performance objectives is necessary to assess the importance of processes and couplings. 


\subsection{IMPORTANCE OF COUPLINGS}

An assessment of the importance of coupled THMC processes is summarized in Tables 4-1, 4-2, and 4-3. Each table is specific for:

- a time period (operation, containment, or isolation),

- anticipated processes and events (see 10 CFR 60.2),

- performance objectives (see 10 CFR 60.111, 60.112, and 60.113; and 40 CFR Part 191), and

- the proposed geologic repository at Yucca Mountain, Nevada.

Each of these specifications narrows the scope and increases the usefulness of this assessment. It is easiest to state that all processes and couplings need to be considered, and there would be no errors in such a statement. However, such a broad statement is not considered useful in identifying the important couplings, as well as the negligible couplings. It is expected that many calculations will be performed, hence it is prudent that resources be directed to the most important processes and couplings. Through a review of process couplings (as summarized in Section 2), and comparison of processes, the most important processes can be identified.

The description of time period, anticipated processes and events, performance objectives, and proposed repository are used in the tables and are considered important in narrowing the scope of the tables. The time periods and performance objectives have been discussed in Section 4.1, and are not discussed further. The other descriptions are discussed here.

In the tables, the term "anticipated processes and events" is used to narrow the scope and is defined in the regulations (10 CFR 60.2): "Anticipated processes and events means those natural processes and events that are reasonably likely to occur during the period the intended performance objective must be achieved." The term "reasonably likely" implies a probability of occurrence which is unspecified in the regulations. Although unspecified, the probability is on the order of at least one occurrence during the time period of interest. Hence, the couplings assessed in the tables are only applied to those processes and events which are reasonably likely to occur.

In the tables, the term "proposed repository" is used to narrow the scope of the tables to concentrate on the couplings pertinent at Yucca Mountain. Hence the tables are not for a generic repository. Previously, other geologic locations were considered as possible repository locations. These included salt, granite, basalt, and shale (Wang et al., 1983). These locations have different mechanical, thermal, hydrologic, and chemical properties, hence different processes and couplings would be expected.

A spatial designation of container, near-field, and far-field was also considered to help clarify where coupled processes may need to be considered. The spatial description would have been in parallel with the temporal description (operation, containment, and isolation). However, the spatial description is closely related to the performance objective, which is related to the principal process. After consideration, the spatial description was not adopted for this work and is considered an implicit part of the performance objectives for each time period. 
Table 4-1. Assessment of the importance of coupled THMC processes for anticipated processes and events, for performance objectives associated with the operations time period of the proposed HLW repository (10 CFR 60.111).

\begin{tabular}{||l|c|c|c|c||}
\cline { 2 - 5 } \multicolumn{1}{c|}{} & Thermal & Hydrologic & MECHANICAL & Chemical \\
\hline \hline Thermal $\Rightarrow$ & N/A & 1 & 1 & 1 \\
\hline Hydrologic $\Rightarrow$ & 2 & N/A & 3 & 3 \\
\hline Mechanical $\Rightarrow$ & 3 & 3 & N/A & 3 \\
\hline Chemical $\Rightarrow$ & 3 & 3 & 2 & N/A \\
\hline
\end{tabular}

Table 4-2. Assessment of the importance of coupled THMC processes for anticipated processes and events, for performance objectives associated with the containment time period at the proposed HLW repository (10 CFR 60.113).

\begin{tabular}{||c|c|c|c|c|}
\cline { 2 - 5 } \multicolumn{1}{c|}{} & Thermal & Hydrologic & Mechanical & CHEMICAL \\
\hline \hline Thermal $\Rightarrow$ & N/A & 1 & 1 & 1 \\
\hline Hydrologic $\Rightarrow$ & 2 & N/A & 2 & 1 \\
\hline Mechanical $\Rightarrow$ & 3 & 2 & N/A & 2 \\
\hline Chemical $\Rightarrow$ & 3 & 3 & 2 & N/A \\
\hline
\end{tabular}

Table 4-3. Assessment of the importance of coupled THMC processes for anticipated processes and events, for performance objectives associated with the isolation time period at the proposed BLW repository (10 CFR 60.112 and 40 CFR Part 191).

\begin{tabular}{||c|c|c|c|c|}
\cline { 2 - 5 } \multicolumn{1}{c|}{} & Thermal & Hydrologic & Mechanical & CHEMICAL \\
\hline \hline Thermal $\Rightarrow$ & N/A & 1 & 2 & 1 \\
\hline Hydrologic $\Rightarrow$ & 2 & N/A & 3 & 1 \\
\hline Mechanical $\Rightarrow$ & 3 & 3 & N/A & 3 \\
\hline Chemical $\Rightarrow$ & 3 & 2 & 2 & N/A \\
\hline
\end{tabular}

Key: Numerical ratings are:

1 = primary - confidently expected to be important;

2 = secondary - potentially important;

$3=$ tertiary - confidently expected to be negligible. .

Note: Two-way and higher-order couplings are implicitly considered as combinations of the one-way couplings explicitly shown in the tables.

Note: The affected process most closely related to regulatory interest is highlighted in all capital letters and underlined in the tables.

Note: The assessments provided in Tables 4-1, 4-2, and 4-3 are subjective and are based on the current understandings of the processes and data uncertainties, and may bave to be modified as new data and information emerge. 
The tables summarize an "assessment of the importance of coupled THMC processes." The term assessment is used to emphasize that this is a summary of the state-of-the-art. Although a large amount of effort was expended in reviewing the literature, it is probable that portions of the literature have not been reviewed. Also, because the scientific knowledge of the processes is continually improving, it is probable that future assessments will be more definitive. Hence, the reader is cautioned that these assessments are likely to be updated. Nonetheless, it is considered important to summarize this review of the literature as best as currently possible in order to guide future work. follow.

Having discussed the scope and caveats of the tables, the information content of the tables will

The tables summarize 12 one-way couplings in parallel with Section 2 of this report (e.g., $\mathrm{T} \Rightarrow \mathrm{H}, \mathrm{T} \Rightarrow \mathrm{M})$. The motivating processes are in the left column, and the influenced processes are in the upper row. The self couplings (i.e., $\mathrm{T} \Rightarrow \mathrm{T}, \mathrm{H} \Rightarrow \mathrm{H}, \mathrm{M} \Rightarrow \mathrm{M}, \mathrm{C} \Rightarrow \mathrm{C}$ ) are not discussed in this context, and are noted to be nonapplicable (N/A).

Within each box in the tables are subjective numerical ratings for the assessed importance of each one-way coupling. The ratings are $1=$ primary, $2=$ secondary, and $3=$ tertiary. The definition of ratings are as follows:

- primary = confidently expected to be important;

- secondary = potentially important, however the available evidence suggests that the coupling may be neglected without significant errors;

- tertiary $=$ confidently expected to be negligible.

Hence in Table 4-1, the $\mathrm{T} \Rightarrow \mathrm{H}$ coupling is considered a "primary" coupling, so that if hydrologic processes are to be studied then thermal processes should also be considered. Similarly, in Table 4-1, the $\mathrm{M} \Rightarrow \mathrm{C}$ coupling is considered "tertiary" and is expected to have negligible influence on chemical processes.

A coupling is designated as being primary if there is a clear consensus of evidence (either from field studies, laboratory studies, or theoretical analyses) that the coupling mechanisms between the processes should not be neglected in a model used to design and/or assess repository performance. If the coupling is neglected, the model can be expected to yield predictions that are substantially different from the true observations. For example, if a hydrologic model neglected thermal processes, then the anticipated dryout zone and anticipated condensate dripping in fracture networks would be missed. A purely isothermal hydrologic model may be adequate for the very far-field hydrologic model (e.g., Ahola and Sagar, 1992), but it is inadequate to predict hydrologic conditions near the waste packages. It is considered especially important to the repository performance that hydrologic processes be accurately predicted during the containment and isolation time periods because hydrologic processes strongly affect the container corrosion processes, container lifetimes, and waste form dissolution processes (i.e., the source term).

A coupling is designated as being secondary if the available evidence suggests that the coupling may be neglected without significant errors. A model that neglects a secondary coupling is expected to predict repository performance with reasonable accuracy. Conversely, if the coupling were to be included, 
the model would not be expected to yield more accurate predictions. Frequently, the secondary coupling mechanisms have not received as much investigation as primary couplings, hence the couplings are considered potentially important. Future studies may improve the understanding of the coupling to indicate whether it should be considered either primary or tertiary.

A particular coupling may be poorly understood, hence a lack of technical information exists in the literature. In this case, it is implicitly assumed that there exists enough information to at least characterize the coupling as either important, potentially important, or negligible. If enough evidence exists to consider the poorly understood coupling to be either primary or secondary, then future studies to improve the state of knowledge are suggested.

A secondary rating of a coupling does not imply that a model will yield excellent agreement between predictions and observations. Frequently there are inherent uncertainties in the physical properties and/or conceptual models, which lead to a distribution of possible predictions and/or observations. For example, it has been widely noted that hydraulic conductivities for the media at Yucca Mountain can vary from two to four orders of magnitude (e.g., Wang, 1992). Similarly, unless data samples are acquired from every location at Yucca Mountain, the physical extent of the different hydrogeologic units (and the related hydrologic properties) must be interpreted from a finite set of sample locations. This introduces the subject of geostatistics and stochastic models. It is expected that models will be developed to explicitly account for the uncertainties in properties, hence the predictions will consist of distributions of expected solutions. Consequently, one is faced with the reality of variabilities and uncertainties in material properties which means that distributions of predictions should be compared with distributions of observations to gauge the level of agreement.

A coupling is designated as being tertiary if there is little to no experimental or theoretical evidence that suggests that the coupling needs to be considered to accurately predict 'observations. It is expected that this coupling can be neglected in a model, and the resulting predictions would be without significant error.

It appears impossible to demonstrate that all truly negligible couplings are negligible (i.e., tertiary) for two reasons: (i) there is an infinite number of negligible couplings, and (ii) a finite amount of resources (time and money) are required to demonstrate that each apparently negligible coupling is truly negligible. It appears unreasonable to presume that all possible couplings must be rigorously shown to be negligible. In contrast, it appears more reasonable to presume that a coupling is negligible unless evidence exists that it is otherwise.

One difficult issue is distinguishing between phenomena that may not be studied because they are either (i) complex and difficult to study, or (ii) truly of negligible importance. Important processes and couplings need to be investigated regardless of their complexity and/or difficulty to study. On the other hand, it appears prudent to require a minimum threshold of evidence before a process or coupling is investigated (i.e., considered non-negligible), versus the contrary where everything is considered important a priori.

The criteria used in this assessment are considered subjective. The development of definitive objective criteria from 10 CFR Part 60 and 40 CFR Part 191 is considered worthwhile, but is beyond the intended scope of this literature review. Justification for each rating is summarized in the earlier sections of this report. For example, the $\mathrm{T} \Rightarrow \mathrm{H}$ coupling is considered a primary coupling during all time periods 
because the current understanding and experimental evidence suggest that the changing temperature field will significantly influence hydrologic conditions (both liquid and vapor phases).

Figure 4-1 graphically summarizes the assessment presented in Tables 4-1 through 4-3. Again, three time periods are considered (operation, containment, and isolation), and the four processes are shown ( $\mathrm{T}, \mathrm{H}, \mathrm{M}$, and $\mathrm{C}$ ), with the processes most closely related to the performance objectives inside a highlighted circle. Primary couplings are indicated with solid lines, secondary couplings with dashed lines, and tertiary couplings are not drawn. In the left column, all four processes are shown with primary and secondary couplings. As a subset, only the primary couplings which influence the process most closely related to regulatory performance interest are shown in the right column. From Figure 4-1, the importance of mechanical processes during operation is highlighted, along with the necessity for considering thermal processes. After the operation time period, chemical processes are most closely related to the performance objectives along with the necessity for considering thermal and hydrologic processes. In comparison, mechanical processes are important during operations, and hydrologic and chemical processes are important during containment and isolation. Thermal processes appear to be important during all the time periods, because they influence mechanical, hydrologic, and chemical processes.

In light of the available experimental and theoretical data, the primary couplings shown in the right column of Figure 4-1 should be considered as the minimum set of couplings which need to be considered for design and in assessing the performance of the repository. Secondary couplings appear to be negligible at this time; however, the possibility exists that future studies and improved understanding may show them to be non-negligible.

A review was conducted to determine if other assessments have been presented in the literature. Although many publications have alluded to the importance of different couplings, very few have attempted to gauge the importance of the couplings for performance assessment, especially in light of the unavoidable uncertainties in data. One of the earliest assessments was reported by Wang et al., (1983) where the focus is primarily on radionuclide transport (see Figure 4-2). In comparison, radionuclide transport is considered as a chemical process in Tables 4-1 through 4-3. Wang et al. concluded that fluid flow is likely to have a small effect on the temperature field $(\mathrm{H} \Rightarrow \mathrm{T})$ which is shown as a smaller arrow in Figure 4-2. Similarly, Wang et al. indicated that the $C \Rightarrow M, M \Rightarrow T$, and $C \Rightarrow T$ couplings are negligible (indicated by the lack of arrows in Figure 4-2). In comparison, Wang et al. do not explicitly assess the importance of coupled processes during each of the time periods, but only once for a generic repository not necessarily at Yucca Mountain. The assessment presented in Tables 4-2 and 4-3 was compared with the assessment of Wang et al. (1983) and found to be in agreement for many of the couplings $(T \Rightarrow H, T \Rightarrow M, T \Rightarrow C, H \Rightarrow T, H \Rightarrow C)$. Differences exist for the $M \Rightarrow H$ and $C \Rightarrow H$ couplings which Wang considers more important. The present assessment is considered more relevant to Yucca Mountain, and benefits from the growth in understanding over the past 10 yrs.

\subsection{SUMMARY AND CONCLUSIONS}

In this section, coupled THMC processes are considered. The importance of studying these processes is related to the regulatory design and/or performance objectives (10 CFR Part 60 , especially Sections 60.111, 60.112, and 60.113; and 40 CFR Part 191). The current understanding of processes and couplings which is presented in Section 2, is synthesized into an overall assessment of the importance of the different couplings. Each coupling is considered for three time periods (operation, containment, isolation), for anticipated processes and events, for the regulatory performance objectives, and for the 


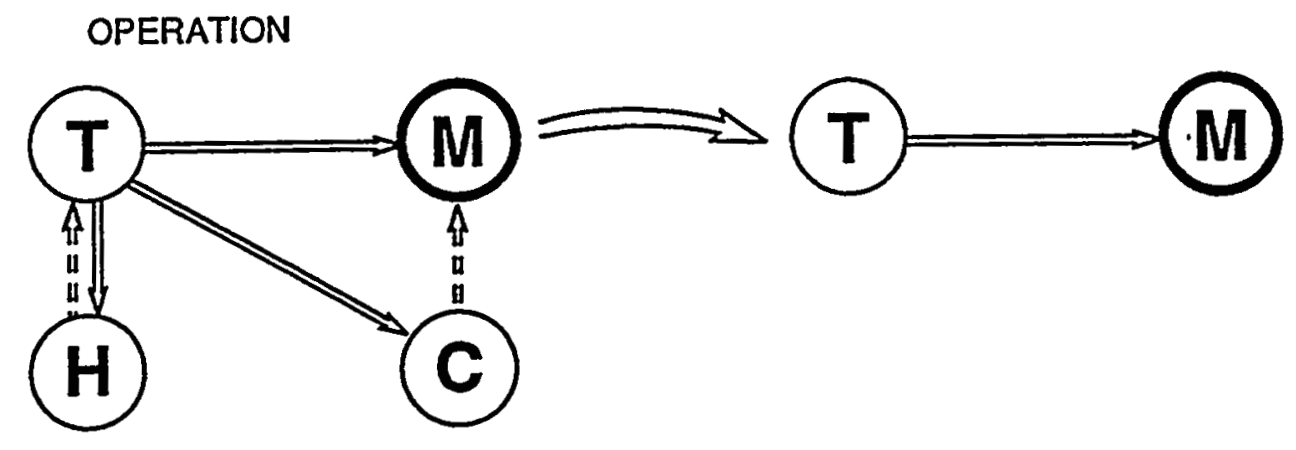

CONTAINMENT

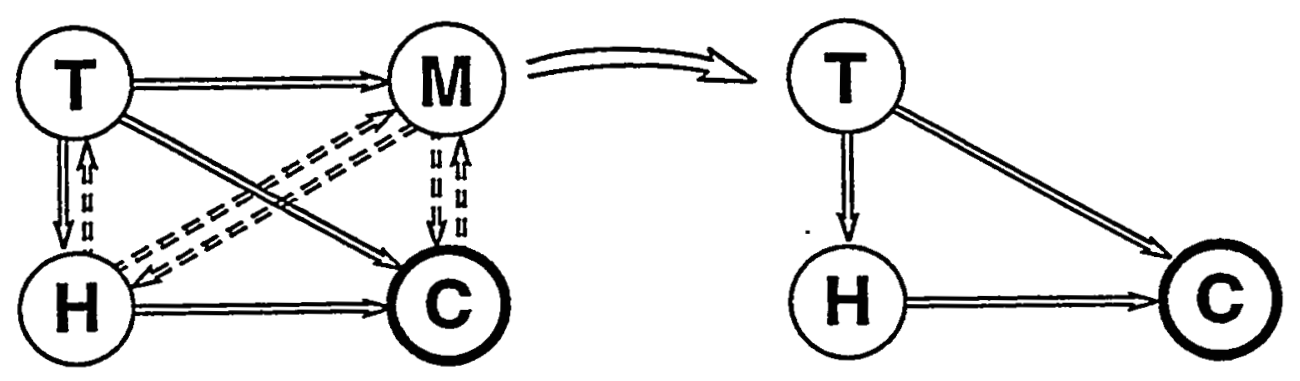

ISOLATION

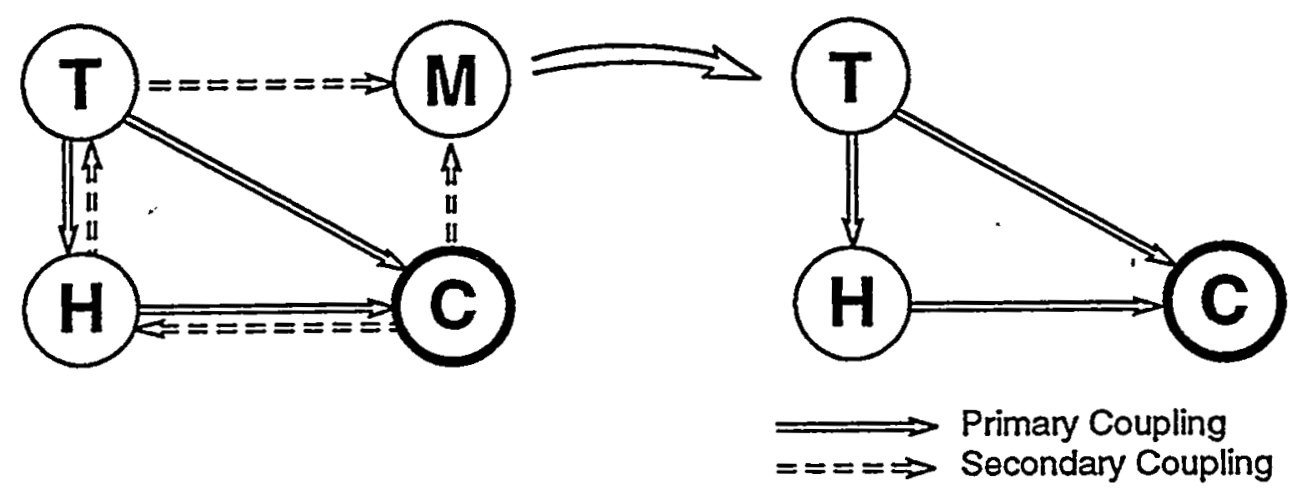

Figure 4-1. Ilustration of the important processes and couplings during the operation, containment, and isolation periods for anticipated processes and events at the proposed repository. The right column is a subset of the left, where only primary couplings which feed into the processes of primary regulatory interest are shown. 
proposed repository at Yucca Mountain. A categorization scheme is presented whereby the coupling mechanisms are considered to be either primary, secondary, or tertiary. Based on the performance objectives outlined in the regulations, mechanical processes are especially important during the operation time period because mechanical processes are most closely related to stability of mined excavations and the retrievability option. Similarly, chemical processes are especially important during the containment and isolation time periods because chemical processes are closely related to corrosion of waste containers, the release of radionuclides, and migration of radionuclides through the geologic setting.

Although the assessment presented here on THMC-coupled processes is necessarily subjective, it provides useful information on focusing future research activities on those specific coupling mechanisms that are considered important. It should be emphasized that this assessment has been based on the current state-of-the-art. As site-characterization studies proceed at Yucca Mountain and scientific knowledge increases, the assessment presented in this report will necessarily be updated.

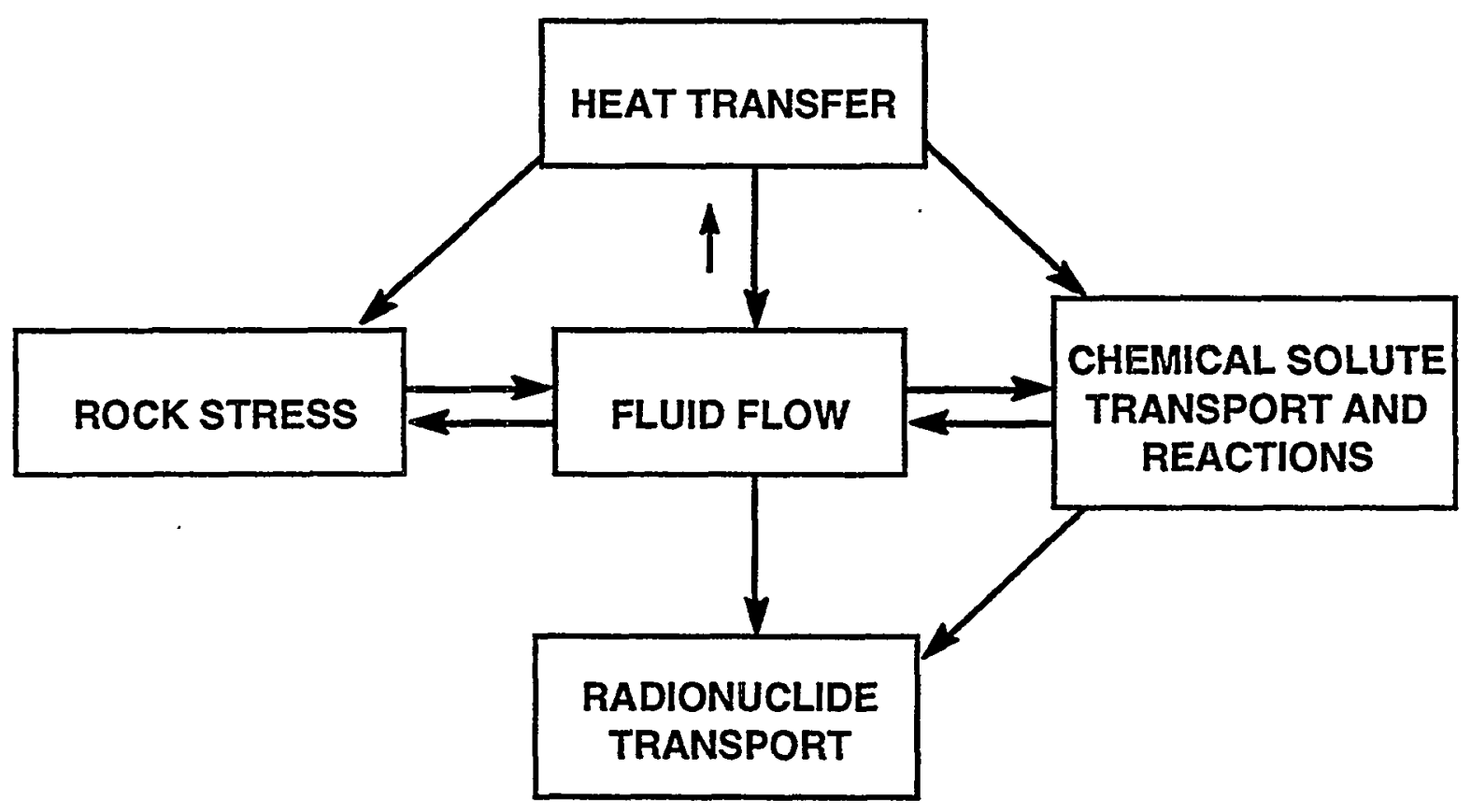

Figure 4-2. Schematic diagram of major factors affecting radionuclide transport from geologic repositories (Wang et al., 1983). 


\section{SUMMARY AND RECOMMENDATIONS}

A literature review has been conducted to establish the state of knowledge relevant to modeling coupled THMC processes as it is pertinent to the proposed HLW repository at Yucca Mountain, Nevada. This assessment attempts to identify potential technical deficiencies in the subject with regard to coupling mechanisms between processes.

This section describes those topics which are of major concern and should be considered in planning future work:

- adequacy of current conceptual models;

- adequacy of current mathematical models and computer codes;

- lack of well-documented coupled-effects experiments;

- need for validation of mathematical models, and verification of computer codes.

The following material is a concise summary of the key findings of this literature search, with particular attention to the issues of concern noted above.

\subsection{ONE-WAY COUPLED PROCESSES}

One-way coupled processes provide a fundamental basis for multiple couplings involving two, three, or more processes. Considering the four kinds of processes $(T, H, M, C)$ the number of possible one-way couplings is twelve. Each one-way coupling was discussed in Section 2. The importance of each one-way coupling within three different time periods of the proposed HLW repository was discussed in Section 4. This assessment is subjective and based on our current knowledge and may have to be modified as new data and information emerge.

The potential influence of the temperature field on hydrologic processes was assessed to be strong. The elevated temperatures in a repository will lead to vaporization of the groundwater. Temperature gradients will provide a driving force for the water vapor to flow into cooler regions. The water vapor has the potential to condense in cooler regions and flow due to both gravity and capillary-pressure gradients. Thus, vaporization, gas flow, condensation, and liquid flow are anticipated to be important processes in affecting the near-field hydrology in the proposed repository at Yucca Mountain. Many of the hydrogeologic units at Yucca Mountain are fractured with a low permeability matrix. Episodic rainfall and infiltration at the ground surface, and water vapor condensation, are two mechanisms whereby the fractures may transmit a substantial amount of liquid. More work, however, needs to be done on the study of such effects in fractured porous systems, which should include characterization of matrix-fracture interactions. Hydrologic processes may also affect thermal processes through a number of coupling mechanisms, the most important one being the gravity-driven condensate drainage in fractures toward the heat source. Mechanical processes can affect hydrologic processes through changes in fracture apertures, which, in turn, affect hydrologic conductivity and fluid storativity.

The potential influence of the temperature field on mechanical response was also assessed to be strong and represents the well-known thermomechanical effects. More work should be done in this area 
to evaluate the impact of existing fractures and joints, and the impact of temperature on mechanical properties and long-term mechanical degradation.

The thermal effects on chemical behavior include temperature effects on the equilibrium constants and kinetics of chemical reactions. At present, only a few codes such as EQ3/6 and PHREEQE are able to fully model reaction processes, and even fewer codes (e.g., EQ3/6) can model reaction kinetics as a function of time. Much of the thermodynamic data, especially for many of the key radionuclides, are only available for low temperatures. The coupled effects of temperature on system chemistry can be significant, and further study should be done.

The hydrologic-chemical coupling includes chemical equilibria, kinetics and mass transport in fractured porous rocks. Interaction between fluids, the rock matrix, and fracture-lining materials should be taken into account. Currently-available computational methods have many limitations and need additional work.

\subsection{THREE-WAY COUPLED PROCESSES}

Among the four processes, there can only be four possible three-way couplings. The THM coupling describes the thermally-induced hydromechanical behavior of fractured rocks, where both heat and fluid pressure may change the fracture apertures, with the result that buoyant flow may be strongly affected. Most of the computer codes available for modeling coupled THM processes have many limitations and are only partially verified against analytical solutions for two-way couplings such as hydromechanical, thermomechanical, and hydrothermal problems. Studies related to partially-saturated media are almost nonexistent and are recommended.

The THC coupling includes chemical reactions and heat and mass transport in rock masses. A chemical reaction could be mineral precipitation (or dissolution), which changes the rock permeability to fluid flow. Such a process has been studied in hydrothermal systems. Other chemical reactions of interest are those involved in degradation of waste packages, including corrosion of containers and dissolution of spent fuel. For problems related to nuclear waste repositories, further quantitative studies are recommended.

\subsection{FOUR-WAY COUPLED PROCESSES}

In a situation where all four processes are interrelated, the coupling is the four-way coupling, THMC. As discussed in Section 4, this coupling is most important during the containment period (i.e., closure until 300 to $1,000 \mathrm{yrs}$ ). This coupling includes chemical reactions, mass transport, and fluid flow subject to a potentially strong thermal loading. The thermal effects may cause mechanical changes in fracture apertures, hence, fracture permeability. Chemical precipitation and dissolution may also change the permeability of the rock mass. These two effects join together to influence the fluid flow and chemical transport.

It is generally assumed that numerical methods will be used extensively for coupled analyses for design and performance assessment of the proposed HLW geological repository. The numerical methods should take into account the coupled behavior considering both time and geometric scales. Essential features of a selected computer code are that it can model the processes and the non-negligible coupling mechanisms, and that the partially-saturated, discontinuous nature of the rock mass be simulated. 
Perhaps the most critical aspect of the application of a computational method is validation of the mathematical models. Development of well-controlled laboratory or field experiments appears to be the most feasible approach for validation of mathematical models, hence, they are recommended. 


\section{REFERENCES}

Acronyms used in references:

$\begin{array}{ll}\text { ACS } & \text { - American Chemical Society } \\ \text { ANS } & \text { - American Nuclear Society } \\ \text { ASME } & \text { - American Society of Mechanical Engineers } \\ \text { CNWRA } & \text { - Center for Nuclear Waste Regulatory Analyses } \\ \text { CSUES } & \text { - Colorado State University Experiment Station } \\ \text { DOE } & \text { - Department of Energy } \\ \text { EPA } & \text { - Environmental Protection Agency } \\ \text { EPRI } & \text { - Electric Power Research Institute } \\ \text { IAEA } & \text { - International Atomic Energy Agency } \\ \text { IMM } & \text { - Institute of Mining and Metallurgy } \\ \text { LANL } & \text { - Los Alamos National Laboratory } \\ \text { LBL } & \text { - Lawrence Berkeley Laboratory } \\ \text { LLNL } & \text { - Lawrence Livermore National Laboratory } \\ \text { MIT } & \text { - Massachusetts Institute of Technology } \\ \text { MRS } & \text { - Materials Research Society } \\ \text { NRC } & \text { - Nuclear Regulatory Commission } \\ \text { NTIS } & \text { - National Technical Information Service } \\ \text { OCRWM } & \text { - Office of Civilian Radioactive Waste Management } \\ \text { ONWI } & \text { - Office of Nuclear Waste Isolation } \\ \text { ORNL } & \text { - Oak Ridge National Laboratory } \\ \text { PNL } & \text { - Pacific Northwest Laboratory } \\ \text { SAIC } & \text { - Science Applications International Corporation } \\ \text { SKB } & \text { - Swedish Nuclear Fuel and Waste Management Company } \\ \text { SKI } & \text { - Swedish Nuclear Power Inspectorate } \\ \text { SME/AIME } & \text { - Society of Mining Engineers of the American Institute of Mining, Metallurgical, and } \\ \text { SNL } & \text { - Petroleum Engineers } \\ \text { USDA } & \text { - U.S. Department of Agriculture } \\ \text { USGS } & \text { - U.S. Geological Survey }\end{array}$

10 CFR Part 60 (Code of Federal Regulations). 1992. Title 10, Energy, Part 60, Disposal of High-Level Radioactive Wastes in Geologic Repositories. Washington, DC: Office of the Federal Register.

40 CFR Part 191 (Code of Federal Regulations). 1989. Title 40, Protection of Environment, Part 191, Environmental Radiation Protection Standards for Management and Disposal of Spent Nuclear Fuel, High-Level and Transuranic Radioactive Wastes. Washington, DC: Office of the Federal Register.

Aagaard, P., and H.C. Helgeson. 1982. Thermodynamic and kinetic constraints on reaction rates among minerals and aqueous solutions. I. Theoretical considerations. American Journal of Science 282: 237-285. 
Ababou, R. 1991. Approaches to Large Scale Unsaturated Flow in Heterogeneous, Stratified, and Fractured Geologic Media. NUREG/CR-5743. Washington, DC: NRC.

ABAQUS. 1988. ABAQUS User's Manual. Pawtucket, RI: Hibbitt, Karlsson \& Sorensen, Inc.

Ahola, M.P., S. Hsiung, L. Lorig, and A.H. Chowdhury. 1992. Thermo-Hydro-Mechanical Coupled Modeling: Multiple Fracture Model, BMT2; Coupled Stress-Flow Model, TC1. DECOVALEXPhase I. CNWRA 92-005. San Antonio, TX: CNWRA.

Ahola, M.P., and B. Sagar. 1992. Regional Groundwater Modeling of the Saturated Zone in the Vicinity of Yucca Mountain, Nevada. CNWRA 92-001. San Antonio, TX: CNWRA.

Alberty, R.A., and F. Daniels. 1979. Physical Chemistry. New York, NY: Wiley.

Alemi, M.H., D.A. Goldhamer, and D.R. Nielsen. 1991. Modeling selenium transport in steady-state, unsaturated soil columns. Journal of Environmental Quality 20: 89-95.

Allison, J.D., D.S. Brown, and K.J. Novo-Gradac. 1990. MINTEQA2/PRODEFA2, A Geochemical Assessment Model for Environmental Systems: Version 3.0 User's Manual. Athens, GA: EPA.

Amadei, B., and R.E. Goodman. 1983. A 3-D constitutive relation for fractured rock masses. B. Amadei (ed.), Rock Anisotropy and the Theory of Stress Measurements. Boulder; CO: University of Colorado, Department of Civil Engineering Lecture Notes in Engineering 2: 267-286.

Amter, S., and B. Ross. 1990. Simulation of gas flow beneath Yucca Mountain, Nevada with a model based on freshwater head. Proceedings of the Symposium on Waste Management. R.G. Post, ed. Tucson, AZ: University of Arizona: 915-925.

Anderson, C.A. 1986. SANGRE: A Finite Element Code for Fluid Migration, Heat Transport, and Faulting in Highly Deformable, Porous Geological Media. LA-10666-MS. Los Alamos, NM: LANL.

ANSYS. 1991. ANSYS Procedures Manual - Volume I. Houston, PA: Swanson Analysis Systems, Inc.

ADINA. 1992. ADINA Theory and Modeling Guide. Watertown, MA: ADINA \&D, Inc.

Archambeau, C.B., and N.J. Price. 1991. An Assessment of J.S. Szymanski's Conceptual Hydrotectonic Model. Minority Report of the Special DOE Review Panel. Washington, DC: DOE.

Arulmoli, K., and C.M. St. John. 1987. Analysis of Horizontal Waste Emplacement Boreholes of a Nuclear Waste Repository in Tuff. SAND86-7133. Albuquerque, NM: SNL.

Atkinson, B.K. 1979. Fracture toughness of Tennessee sandstone and Carrera marble using the double beam torsion testing method. International Journal of Rock Mechanics and Mineral Science and Geomechanical Abstracts 16: 49-53. 
Atkinson, B.K., and P.G. Meredith. 1987. The theory of subcritical crack growth with applications to minerals and rocks. Fracture Mechanics of Rock. B.K. Atkinson, ed. New York, NY: Academic Press.

Baes, C.F., and R.E. Mesmer. 1976. The Hydrolysis of Cations. New York, NY: Wiley.

Bagtzoglou, A.C., A.F.B. Tompson, and D.E. Dougherty. 1992a. Projection functions for particle-grid methods. Numerical Methods for Partial Differential Equations 8: 325-340.

Bagtzoglou, A.C., G.W. Wittmeyer, R. Ababou, and B. Sagar. 1992b. Application of a massively parallel computer to flow in a variably saturated heterogeneous porous media. T.F. Russell et al., eds. Computational Methods in Water Resources IX. Vol. 1: Numerical Methods in Water Resources. Boston, MA: Computational Mechanics Publications: 695-703.

Ball, J.W., and D.K. Nordstrom. 1991. User's Manual for WATEQ4F, with Revised Thermodynamic Data Base and Test Cases for Calculating Speciation of Major, Trace, and Redox Elements in Natural Waters. USGS-OFR-91-183, Menlo Park, CA: USGS.

Ball, J.W., E.A. Jenne, and D.K. Nordstrom. 1979. Chemical Modeling in Aqueous Systems. Speciation, Sorption, Solubility, and Kinetics. E.A. Jenne, ed. ACS Symposium Series 93. Washington, DC: ACS: 815-835.

Ball, J.W., D.K. Nordstrom, and D.W. Zachmann. 1987. WATEQ4F-A Personal Computer FORTRAN Translation of the Geochemical Model WATEQ2 with Revised Database. USGS-OFR-87-50. Reston, VA: USGS.

Bandis, S.C., A.C. Lumsden, and N.R. Barton. 1983. Fundamentals of rock joint deformation. International Journal of Rock Mechanics and Mineral Science and Geomechanical Abstracts 20: 249-268.

Banerjee, P.K., and S. Ahmad. 1985. Advanced three-dimensional dynamic analysis by boundary element methods. Advanced Topics in Boundary Element Analysis. T.A. Cruse, et al., eds. New York, NY: ASME 72: 65-81.

Bard, A.J., R. Parsons, and J. Jordan, eds. 1985. Standard Potentials in Aqueous Solutions. International Union of Pure and Applied Chemistry Series. New York, NY: Marcel Dekker.

Barry, D.A. 1990. Supercomputers and their use in modeling subsurface solute transport. Reviews of Geophysics 28(3): 277-295.

Barton, N.R., and S.C. Bandis. 1982. Effects of block size on the shear behavior of jointed rock. 23rd U.S. Symposium on Rock Mechanics. Littleton, CO: SME/AIME.

Barton, N.R., S.C. Bandis, and K. Bakhtar. 1985. Strength, deformation and conductivity coupling of rock joints. International Journal of Rock Mechanics and Mineral Science and Geomechanical Abstracts 22(3): 121-140. 
Bauer, S.J., and L.S. Costin. 1990. Thermal and Mechanical Codes First Benchmark Exercise Part II: Elastic Analysis. SAND89-0757. Albuquerque, NM: SNL.

Bauer,S.J., M.P. Hardy, and M. Lin. 1991. Predicted thermal and stress environments in the vicinity of repository openings. Proceedings of the International High-Level Radioactive Waste Management Conference. La Grange Park, IL: ANS: 564-571.

Bear, J. 1972. Dynamics of Fluids in Porous Media. New York, NY: Dover.

Bear, J., and Y. Bachmat. 1991. Introduction to Modeling of Transport Phenomena in Porous Media. Norwell, MI: Kluwer Academic Publishers Group.

Bear, J., and A. Verruijt. 1987. Modeling Groundwater Flow and Pollution. Dordrecht, Holland: D. Reidel Publishing Company.

BEASY. 1993. BEASY Boundary Element Analysis System, Version 4.1. Billerica, MA.: Computational Mechanics, Inc.

Bejan, A. 1984. Convection Heat Transfer. New York, NY: Wiley.

Benson, L.V., and L.S. Teague. 1980. A Tabulation of Thermodynamic Data for Chemical Reactions Involving 58 Elements Common to Radioactive Waste Package Systems. LBL-11448. Berkeley, CA: LBL.

Bethke, C. 1992. The Geochemist's Workbench. ${ }^{\mathrm{TM}}$ A Users Guide to Rxh, Act 2, Tact, React, Gtplot. Urbana-Champaign, IL: University of Illinois.

Bettess, P., and O.C. Zienkiewicz. 1977. Diffraction and refraction and surface waves using finite and infinite elements. Intemational Journal for Numerical Methods in Engineering 11: 1271-1290.

Bian, S.H., M.J. Budden, and S.C. Yung. 1987. Application of GEOTHER/TT to Assess Groundwater Transients in a High-Level Radioactive Waste Repository. PNL-SA-14502. Richland, WA: PNL.

Bian, S.H., M.J. Budden, C.L. Bartley, and S.C. Yung. 1988a. GEOTHER Evaluation and Improvement: A Progress Report Including Test Cases for Two-Dimensional BWIP Analysis. PNL-6245. Richland, WA: PNL.

Bian, S.H., M.J. Budden, C.L. Bartley, and S.C. Yung. 1988b. GEOTHER/VT4: A Two-Phase Groundwater Fluid Flow and Heat Transport Code for High-Level Radioactive Waste Applications. PNL-6517. Richland, WA: PNL.

Bidner, M.S., and V.C. Vampa. 1989. A general model for convection-dispersion-dynamic adsorption in porous media with stagnant volume. Journal of Petroleum Science and Engineering 3: 267-281.

Biot, M.A. 1941. General theory of three-dimensional consolidation. Journal of Applied Physics 12: 144164. 
Bird, R.B., W.E. Stewart, and E.N. Lightfoot. 1960. Transport Phenomena. New York, NY: Wiley.

Bischoff, J.L. 1991. Densities of liquids and vapors in boiling $\mathrm{NaCl}-\mathrm{H}_{2} \mathrm{O}$ solutions: A PVTX summary from $300^{\circ}$ to $500^{\circ} \mathrm{C}$. American Journal of Science 291: 309-338.

Bish, D.L. 1986. Evaluation of Past and Future Alterations in Tuff at Yucca Mountain, Nevada, Based on the Clay Mineralogy of Drill Cores USW G-1, G-2, \& G-3. LA-10667-MS. Los Alamos, NM: LANL.

Bixler, N.E. 1985. NORIA-A Finite Element Computer Program for Analyzing Water, Vapor, Air, and Energy Transport in Porous Media. SAND84-2057. Albuquerque, NM: SNL.

Board, M. 1989a. FLAC (Fast Lagrangian Analysis of Continua) Version 2.20. NUREG/CR-5430. Washington DC: NRC.

Board, M. 1989b. UDEC (Universal Distinct Element Code) Version ICGI.5. NUREG/CR-5429. Volume 2. Washington DC: NRC.

Bond, W.J., B.N. Gardiner, and D.E. Smiles. 1982. Constant-flux adsorption of a tritiated calcium chloride solution by a clay soil with anion exclusion. Soil Science Society of America Journal 46: 11331137.

Bond, W.J., and I.R. Phillips. 1990. Approximate solutions for cation transport during unsteady, unsaturated soil water flow. Water Resources Research 26(9): 2195-2205.

Bond, W.J., and P.J. Wierenga. 1990. Immobile water during solute transport in unsaturated sand column. Water Resources Research 26(10): 2475-2481.

Boozer, G., K. Killer, and S. Serdengecti. 1963. Effects of pore fluid on the deformational behavior of rocks subject to triaxial compression. 5th Symposium on Rock Mechanics. New York: Pergamon Press: 579-625.

Bosma, W.J.P., and S.E.A.T.M. van der Zee. 1993. Transport of reacting solute in a one-dimensional, chemically heterogeneous porous medium. Water Resources Research 29: 117-131.

Bowers, T.S., and H.C. Helgeson. 1983. Calculation of the thermodynamic and geochemical consequences of nonideal mixing in the system $\mathrm{H}_{2} \mathrm{O}-\mathrm{CO}_{2}-\mathrm{NaCl}$ on phase relations in geologic systems: equations of state for $\mathrm{H}_{2} \mathrm{O}-\mathrm{CO}_{2}-\mathrm{NaCl}$ fluids at high pressures and temperatures. Geochimica et Cosmochimica Acta 47: 1247-1275.

Brady, B.H.G., and E.T. Brown. 1985. Rock Mechanics for Underground Mining..London: George Allen \& Unwin.

Brandl, H. 1981. Alteration of soil parameters by stabilization with lime. Proceedings of the Eleventh International Conference of Soil Mechanics and Foundation Engineering. Stockholm, Sweden: SKI: 587-595. 
Brebbia, C.A. 1978. The Boundary Element Method for Engineers. New York, NY: Wiley.

Bresler, E., and G. Dagan. 1981. Convective and pore scale dispersive solute transport in unsaturated heterogeneous fields. Water Resources Research 17(6): 1683-1693.

Brown, D.S., and J.D. Allison. 1987. MINTEQAI, An Equilibrium Metal Speciation Model: User's Manual. EPA/600/3-87/012. Athens, GA: EPA.

Brown, P.L., A. Haworth, S.M. Sharland, and C.J. Tweed. 1991. HARPHRQ: A Geochemical Speciation Program Based on PHREEQE. NSS-R.188. Oxon, United Kingdom: Harwell Laboratory.

Broxton, D.E., D.L. Bish, and R.G. Warren. 1987. Distribution and chemistry of diagenetic minerals at Yucca Mountain, Nye County, NV. Clays Clay Mineral 35: 89-110.

Buscheck, T.A., and J.J. Nitao. 1988. Preliminary Scoping Calculations of Hydrothermal Flow in Variably Saturated, Fractured, Welded Tuff during the Engineered Barrier Design Test at the Yucca Mountain Exploratory Shaft Test Site. UCID-21571. Livermore, CA: LLNL.

Buscheck, T.A., and J.J. Nitao. 1992. The impact of thermal loading on repository performance at Yucca Mountain. Proceedings of the International High-Level Radioactive Waste Management Conference. La Grange Park, IL: ANS: 1003-1017.

Callahan, G.D., A.F. Fossum, and D.K. Svalstad. 1989. Documentation of Spectrom-32: A Finite Element Thermomechanical Stress Analysis Program. Volume 1. DOE/CH/10378-2. Washington DC: DOE.

Cameron, D.R., and A. Klute. 1977. Convective-dispersive solute transport with a combined equilibrium and kinetic adsorption model. Water Resources Research 13(1): 183-188.

Carlsson, H., L. Carlsson, and R. Pusch. 1989. Rock Quality Designation of the Hydraulic Properties in the Near Field of a Final Repository for Spent Fuel. SKB Technical Report 89-21. Stockholm, Sweden: SKB.

Carnahan, C.L. 1984a. Thermodynamically coupled mass transport processes in a saturated clay. Scientific Basis for Nuclear Waste Management - VIII. C.M. Jantzen, J.A. Stone, and R.C. Ewing, eds. Pittsburgh, PA: MRS: 491-498.

Carnahan, C.L. 1984b. Thermodynamic coupling of heat and matter flows in near-field regions of waste repositories. Scientific Basis for Nuclear Waste Management - VII. G.L. McVay, ed. Pittsburgh, PA: MRS: 1023-1030.

Carnahan, C.L. 1987a. Effects of coupled thermal, hydrological and chemical processes on nuclide transport. Proceedings of the GEOVAL 1987 Symposium in Stockholm, Volume 2. Stockholm, Sweden: SKI: 493-506.

Camahan, C.L. 1987b. Simulation of chemically reactive solute transport under conditions of changing temperature. Coupled Processes Associated with Nuclear Waste Repositories. C.F. Tsang, ed. San Diego, CA: Academic Press: 249-257. 
Carnahan, C.L. 1987c. Simulation of uranium transport with variable temperature and oxidation potential: The computer program THCC. Scientific Basis for Nuclear Waste Management - X. J.K. Bates and W.B. Seefeldt, eds. MRS: Pittsburgh, PA: 713-721.

Carnahan, C.L. 1988. Some effects of data base variations on numerical simulations of uranium migration. Radiochimica Acta 44/45: 349-354.

Carnahan, C.L. 1990. Coupling of precipitation/dissolution reactions to mass diffusion via porosity changes. Chemical Modeling of Aqueous Systems II. D.C. Melchior and R.L. Bassett, eds. ACS Series 416. Washington, DC: ACS: 234-242.

Carrigan, C.R, G.C.P. King, G.E. Barr, and N.E. Bixler. 1991. Potential for water-table excursions induced by seismic events at Yucca Mountain, NV. Geology 19: 1157-1160.

Cathles, L.M. 1983. An analysis of the hydrothermal system responsible for massive sulfide deposition in the Hokuroku Basin of Japan. Economic Geology, Mono. 5: 439-487.

Cederberg, G.A. 1985. TRANQL: A Ground-Water Mass-Transport and Equilibrium Chemistry Model for Multicomponent Systems. Ph.D. Dissertation. Stanford, CA: Stanford University.

Cederberg, G.A., R.L. Street, and J.O. Leckie. 1985. A groundwater mass transport and equilibrium chemistry model for multicomponent systems. Water Resources Research 21(8): 1095-1104.

Charlez, P.A. 1991. Rock Mechanics-Theoretical Fundamentals Volume 1. Paris, France: Editions Technip.

Chase, M.W., Jr., C.A. Davies, J.R. Downey, Jr., D.J. Frurip, R.A. McDonald, and A.N. Syverud. 1985a. JANAF thermochemical tables. 3rd Ed. Part I (Al-Co). Journal of Physical and Chemical Reference Data 14(1): 1-926.

Chase, M.W., Jr., C.A. Davies, J.R. Downey, Jr., D.J. Frurip, R.A. McDonald, and A.N. Syverud. 1985b. JANAF thermochemical tables. 3rd Ed. Part II (Cr-Zr). Journal of Physical and Chemical Reference Data 14(1): 927-1856.

Chen, E.P. 1986. Two-dimensional continuum model for jointed media with orthogonal sets of joints. 27th U.S. Symposium on Rock Mechanics. SME/AIME: 862-867.

Chen, E.P. 1990. A constitutive model for jointed rock mass with two intersecting sets of joints. Proceedings, International Conference on Jointed and Faulted Rock. Vienna, Austria: A.A. Balkema.

Cheng, P. 1978. Heat transfer in geothermal systems. Advances in Heat Transfer 14: 1-105.

Chesnut, D.A. 1992. Characterizing the altered zone at Yucca Mountain: The beginning of a testing strategy. Proceedings of the International High-Level Radioactive Waste Management Conference. La Grange Park, IL: ANS: 1026-1039. 
Christianson, M.C., and B. Brady. 1989. Analysis of Alternative Waste Isolation Concepts. NUREG/CR5389. Washington, DC: NRC.

Chrysikopoulos, C.V., P.K. Kitanidis, and P.V. Roberts. 1990. Analysis of one-dimensional solute transport through porous media with spatially variable retardation factor. Water Resources Research 26: 437-446.

Chrysikopoulos, C.V., P.K. Kitanidis, and P.V. Roberts. 1992. Macrodispersion of sorbing solutes in heterogeneous porous formations with spatially periodic retardation factor and velocity field. Water Resources Research 28(6): 1517-1529.

Chung, P.A. Yoshitake, G. Cragnolino, and D.D. Macdonald. 1985. Environmentally controlled crack growth rate of type 304 stainless steel in high temperature sulfate solutions. Corrosion 41: 159168.

Cleary, R.W., and M.J. Ungs. 1978. Groundwater Pollution and Hydrology, Mathematical Models and Computer Programs. Report 78-WR-15. Princeton, NJ: Water Resources Program, Princeton University.

Clemo, T.M., J.D. Miller, L.C. Hull, and S.O. Magnuson. 1990. FRAUC-UNIX Theory and User's Manual. EGG-EP-9029. Idaho Falls, ID: EG\&G Idaho.

Cline, J.S., R.J. Bodnar, and J.D. Rimstidt. 1992. Numerical simulation of fluid flow and silicate transport and deposition in boiling hydrothermal solutions: applications to epithermal gold deposits. Journal of Geophysical Research 97(B6): 9085-9103.

Clothier, B.E. 1984. Solute travel times .during trickle irrigation. Water Resources Research 20(12): 1848-1852.

Coats, K.H., and B.D. Smith. 1964. Dead-end pore volume and dispersion in porous media. Society of Petroleum Engineering Journal 4: 073-84.

Codell, R.B., and W.M. Murphy. 1992. Geochemical model for ${ }^{14} \mathrm{C}$ transport in unsaturated rock. Proceedings of the Intermational High-Level Radioactive Waste Management Conference. La Grange Park, IL: ANS: 1959-1965.

Conca, J. 1990. Transport in unsaturated flow systems using centrifuge techniques. Proceedings of the DOE/Yucca Mountain Site Characterization Project Radionuclide Adsorption Workshop at Los Alamos National Laboratory. LA-12325-C. Los Alamos, NM: LANL.

Cook, R.D. 1981. Concepts and Applications of Finite Element Analysis. New York, NY: Wiley.

Copson, H.R. 1959. Effect of composition on stress corrosion cracking of some alloys containing nickel. T.N. Rhodin, ed. Physical Metallurgy of Stress Corrosion Fracture. New York, NY: Interscience.

Cordfunke, E.H.P., and P.A.G. O'Hare. 1978. The Chemical Thermodynamics of Actinide Elements and Compounds. Part 3. Miscellaneous Actinide Compounds. Vienna, Austria: IAEA. 
Costin, L.S., and E.P. Chen. 1991. A thermomechanical analysis of the G-tunnel heated block experiment using a compliant-joint rock-mass model. International Journal for Numerical and Analytical Methods in Geomechanics 15: 379-398.

Cox, J.D., D.D. Wagman, and V.A. Medvedev, eds. 1989. CODATA Key Values for Thermodynamics. New York, NY: Hemisphere.

Cragnolino, G., and N. Sridhar. 1991. A Review of Localized Corrosion of High-Level Nuclear Waste Container Materials-I. CNWRA 91-004. San Antonio, TX: CNWRA.

Cragnolino, G., and N. Sridhar. 1992. A Review of Stress Corrosion Cracking of High-Level Nuclear Waste Container Materials-I. CNWRA 92-021. San Antonio, TX: CNWRA.

Criscenti, L.J., M.L. Kemner, R.L. Erikson, C.J. Hostetler, J.R. Morrey, and J.S. Fruchter. 1989. The FASTCHEM ${ }^{T M}$ Workstation for Integrating Pre-and Postprocessing Functions. EPRI-EA-5871. Palo Alto, CA: EPRI.

Cross, J.E., and F.T. Ewart. 1991. HATCHES-A thermodynamic database and management system. Radiochimica Acta 52/53: 421-422.

Cundall, P.A. 1971. A computer model for simulating progressive, large-scale movement in blocky rock systems. Proceedings of the International Symposium on Rock Fracture II. Nancy, France: II-8.

Cundall, P.A. 1988. Formulation of a three-dimensional distinct element model Part I. A scheme to detect and represent contacts in a system composed of many polyhedral blocks. International Journal of Rock Mechanics and Mineral Science and Geomechanical Abstracts 25(3): 106-107.

Cundall, P.A., and R.D. Hart 1989. Numerical modeling of discontinua. 1st U.S. Conference on Discrete Element Methods. G.G.W. Mustoe, et al., eds. Golden, CO: Colorado School of Mines Press.

Cundall, P.A., and J.V. Lemos. 1988. Numerical simulation of fault instabilities with the continuouslyyielding joint model. Proceedings of the Symposium on Rockbursts and Seismicity in Mines. Charles Fairhurst, ed. University of Minnesota. Rotterdam, Netherlands: A.A. Balkema: 147-152.

Cvetkovic, V.D., and A.M. Shapiro. 1990. Mass arrival of sorptive solute in heterogeneous porous media. Water Resources Research 26(9): 2057-2067.

Dagan, G. 1988. Time-dependent macrodispersion for solute transport in anisotropic, heterogeneous aquifers. Water Resources Research 24: 1491-1500.

Dagan, G. 1986. Statistical theory of groundwater flow and transport: pore to laboratory, laboratory to formation, and formation to regional scale. Water Resources Research 22(9): 120S-134S.

Daily, W., W. Lin, and T. Buscheck. 1987. Hydrological properties of Topopah Spring tuff: laboratory measurements. Journal of Geophysical Research 92: 7854-7864. 
Davis, J.A., and D.B. Kent. 1990. Surface complexation modeling in aqueous geochemistry. Reviews in Mineralogy, Volume 23: Mineral-Water Interface Geochemistry. M.F. Hochella, Jr., and A.F. White, eds. Mineralogical Society of America: Washington, DC: 177-260.

de Donder, T., and P. van Rysselberghe. 1936. Thermodynamic Theory of Affinity: A Book of Principles. Stanford, CA: Stanford University Press.

de Marsily, G. 1986. Quantitative Hydrogeology, Groundwater Hydrology for Engineers. New York, NY: Academic.

de Smedt, F., and P.J. Wierenga. 1979. Mass transfer in porous media with immobile water. Journal of Hydrology 41: 59-67.

de Smedt, F., and P.J. Wierenga. 1984. Solute transfer through columns of glass beads. Water Resources Research 20(2): 225-232.

de Vries, D.A. 1963. Thermal properties of soils. Physics of Plant Environment. W.R. Van Wijk, ed. Amsterdam: North-Holland Publishing Company.

de Vries, D.A. 1975. Heat transfer in soils. Heat and Mass Transfer in the Biosphere. D.A. de Vries and N.N. Afgan, eds. Washington, DC: Scripta Book Co.: 5-28.

Dewers, T., and P. Ortoleva. 1990a. A coupled reaction/transport/mechanical model for intergranular pressure solution, stylolites, and differential compaction and cementation in clean sandstones. Geochimica et Cosmochimica Acta 54: 1609-1625.

Dewers, T., and P. Ortoleva. 1990b. Geochemical self-organization III: A mean field, pressure solution model of metamorphic differentiation. American Journal of Science 290: 473-521.

DOE (U.S. Department of Energy). 1988. Site Characterization Plan: Yucca Mountain Site, Nevada Research and Development Area, Nevada. DOE/RW-0199. Washington, D.C: OCRWM.

DOE (U.S. Department of Energy). 1990. Yucca Mountain Project Reference Information Base. YMP/CC0002 (Version 04.002). Las Vegas, NV: DOE.

Domenico, P.A., and F.W. Schwarz. 1990. Physical and Chemical Hydrogeology. New York, NY: Wiley.

Donaldson, I.G. 1968. The flow of steam water mixtures through permeable beds: a simple simulation of a natural undisturbed hydrothermal region. New Zealand Journal of Science 11: 3-23.

Doughty, C., and K. Pruess. 1988. A semianalytical solution for heat-pipe effects near high-level nuclear waste packages buried in partially saturated geological media. International Joumal of Heat and Mass Transfer 31(1): 79-90.

Doughty, C., and K. Pruess. 1990. A similarity solution for two-phase fluid and heat flow near high-level nuclear waste packages emplaced in porous media. International Journal of Heat and Mass Transfer 33(6): 1205-1222. 
Doughty, C., and K. Pruess. 1991. A Mathematical Model for Two-Phase Water, Air, and Heat Flow around a Linear Heat Source Emplaced in a Permeable Medium. LBL-30050. Berkeley, CA: LBL.

Doughty, C., and K. Pruess. 1992. A similarity solution for two-phase water, air, and heat flow near a linear heat source in a porous medium. Journal of Geophysical Research 97(B2): 1821-1838.

Drever, J.I. 1982. The Geochemistry of Natural Waters. Englewood Cliffs, NJ: Prentice-Hall.

Duncan, J.M., and R.E. Goodman. 1968. Finite Element Analysis of Slopes in Jointed Rocks. U.S. Army Corps of Engineers Report TR 1-68.

Dunn, P.D., and D.A. Reay. 1982. Heat Pipes. New York, NY: Pergamon Press.

Dunning, J.D., W.L. Lewis, and D.E. Dunn. 1980. Chemo-mechanical weakening in the presence of surfactants. Journal of Geophysical Research 85(B10): 5344-5354.

Dunning, J.D., D. Petroviski, J. Schuyler, and A. Owens. 1984. The effects of aqueous chemical environments on crack propagation in quartz. Journal of Geophysical Research 89(B6): 41154123.

Dykhuizen, R.C. 1987. Transport of solutes through unsaturated fractured media. Water Research 21(12): 1531-1539.

Dykhuizen, R.C. 1990. A new coupling term for dual-porosity models. Water Resources Research 26(2): 351-356.

Eckert, E.R.G., and R.M. Drake, Jr. 1987. Analysis of Heat and Mass Transfer. New York, NY: Hemisphere.

Edlefsen, N.E., and A.B.C. Anderson. 1943. Thermodynamics of soil moisture. Hilgardia 15(2): 31-298.

Ellis, A.J., and W.A.J. Mahon. 1977. Chemistry and Geothermal System. New York, NY: Academic Press.

Elrashidi, M.A., and W.L. Lindsay. 1984. Fluorine Supplement to Technical Bulletin 134: Selection of Standard Free Energies of Formation for Use in Soil Chemistry. Technical Bulletin 134 (supplement). Fort Collins, CO: CSUES.

Erikson, R.L., C.J. Hostetler, and M.L. Kemner. 1990. Mobilization and Transport of Uranium at Uranium Mill Tailings Disposal Sites. NUREG/CR-5169. Washington, DC: NRC.

Farmer, J.C., R.A. van Konynenburg, R.D. McCright, and D.B. Bullen. 1988a. Survey of Degradation Modes of Candidate Materials for High-Level Radioactive-Waste Disposal Canisters. Volume 3: Localized Corrosion and Stress Corrosion Cracking of Austenitic Alloys. UCID-21362. Livermore, CA: LLNL. 
Farmer, J.C., R.A. van Konynenburg, R.D. McCright, and G.E. Gdowski. 1988b. Survey of Degradation Modes of Candidate Materials for High-Level Radioactive-Waste Disposal Canisters. Volume 4: Stress Corrosion Cracking of Copper-Based Alloys. UCID-21362. Livermore, CA: LLNL.

Felmy, A.R., D. Girvin, and E.A. Jenne. 1983. MINTEQ: A Computer Program for Calculating Aqueous Geochemical Equilibria. Washington, DC: EPA.

Fleming, G.W., and L.N. Plummer. 1983. PHRQINPT-An Interactive Computer Program for Constructing Input Data Sets to the Geochemical Simulation Program PHREEQE. Water Resources Investigations Report 83-4236. Reston, VA: USGS.

Flint, L.E., and A.L. Flint. 1990. Preliminary Permeability and Water-Retention Data for Nonwelded and Bedded Tuff Samples, Yucca Mountain Area, NYE County, Nevada. USGS Report 90-569. Denver, CO: USGS.

Fontana, M.G., and N.D. Greene. 1978. Corrosion Engineering. New York, NY: McGraw-Hill.

Forster, C., and L. Smith. 1990. Fluid flow in tectonic regimes. Short Course on Fluids in Tectonically Active Regimes of the Continental Crust. B.E. Nesbitt, ed. Nepean, Ontario, Canada: Mineralogical Association of Canada: 1-47.

Forsyth, P.A. 1990. Radioactive waste disposal heating effects in unsaturated fractured rock. Numerical Heat Transfer. 17: 29-51.

Forsyth, P.A., and R.B. Simpson. 1991. A two-phase, two-component model for natural convection in a porous medium. International Journal for Numerical Methods in Fluids 12: 655-682.

Forsythe, G.E., and W.R. Warsow. 1960. Finite Difference Methods for Partial Differential Equations. New York, NY: Wiley.

Fossum, A.F. 1985. Effective elastic properties for a randomly jointed rock mass. International Journal of Rock Mechanics and Mineral Science and Geomechanical Abstracts 22(6): 467-470.

Fox, R.W., and A.T. McDonald. 1978. Introduction to Fluid Mechanics. New York, NY: McGraw-Hill.

Freeze, R.A., and J.A. Cherry. 1979. Groundwater. Englewood Cliffs, NJ: Prentice-Hall.

Friedly, J.C., and J. Rubin. 1992. Solute transport with multiple equilibrium-controlled or kinetically controlled chemical reactions. Water Resources Research 28(6): 1935-1953.

Fuger, J., and F.L. Oetting. 1976. The Chemical Thermodynamics of Actinide Elements and Compounds. Part 2. The Actinide Aqueous Ions. Vienna, Austria: IAEA.

Gallegos, D.P., S.G. Thoma, and D.M. Smith. 1992. Impact of fracture coating on the transfer of water across fracture faces in unsaturated media. Proceedings of the International High-Level Radioactive Waste Management Conference. La Grange Park, IL: ANS: 728-745. 
Garrels, R.M., and C.L. Christ. 1965. Solutions, Minerals, and Equilibria. San Francisco, CA: Freeman, Cooper and Company.

Garven, G., and R.A. Freeze. 1984a. Theoretical analysis of the role of groundwater flow in the genesis of stratabound ore deposits. I. Mathematical and numerical model. American Journal of Science 284: $1085-1124$.

Garven, G., and R.A. Freeze. 1984b. Theoretical analysis of the role of groundwater flow in the genesis of stratabound ore deposits. II. Quantitative results. American Journal of Science 284: 1125-1174.

Gerrard, C.M. 1982. Elastic models of rock masses naming one, two and three sets of joints. International Journal of Rock Mechanics and Mineral Science and Geomechanical Abstracts 19: 15-23.

Gillham, R.W., E.A. Sudicky, J.A. Cherry, and E.O. Frind. 1984. An advection-diffusion concept for solute transport in heterogeneous unconsolidated geological deposits. Water Resources Research 20(3): 369-378.

Glynn, P.D., P. Engesgaard, and K. Kipp. 1991. Two geochemical mass transport codes: PHREEQM and MST1D, their use and limitations at the Pinal Creek toxic waste site. Proceedings of the 10th Meeting of the U.S. Geological Survey Toxic Substance Hydrology Program. Water Resources Investigations Report 91-4034. Monterrey, CA: USGS.

Goldberg, S., H.S. Forster, and E.L. Heick. 1991. Flocculation of illite/kaolinite and illite/montmorillonite mixtures as affected by sodium adsorption ratio and pH. Clays Clay Minerals 39: 375-380.

GPBEST. 1992. GPBEST General Purpose Boundary Element Software Technology: A Comprehensive Engineering Analysis System. Buffalo, NY: Boundary Element Software Technology Corporation.

Green, R.T., R.D. Manteufel, F.T. Dodge, and S.J. Svedeman. 1992. Theoretical and Experimental Investigation of Thermohydrologic Processes in a Partially Saturated, Fractured Porous Medium: A Summary of Work Through December 1991. CNWRA 92-006. San Antonio, TX: CNWRA.

Grenthe, I., J. Fuger, R.J. Lemire, A.B. Muller, C. Nguyen-Trung, and H. Wanner. 1992. Chemical Thermodynamics Series, Volume 1: Chemical Thermodynamics of Uranium. New York, NY: Elsevier.

Griffith, A. 1920. The phenomena of rupture and flow in solids. Philosophical Transactions. Royal Society of London. Series A. Mathematical and Physical Sciences 221: 163-197.

Gureghian, A.B., and B. Sagar. 1991. Evaluation of DCM3D-a dual continuum, 3-D groundwater flow code for unsaturated, fractured, porous media. Report on Research Activities for Calendar Year 1990. W.C. Patrick, ed. NUREG/CR-5817. Washington, DC: NRC.

Guy, C., and J. Schott. 1992. Modeling of soret diffusion in radioactive waste glass. Applied Geochemistry Supplement 1: 33-40. 
Hadley, G.R. 1985. PETROS-A Program for Calculating Transport of Heat, Water, Vapor and Air Through a Porous Material. SAND-0878. Albuquerque, NM: SNL.

Hahn, J.K. 1988. Realistic animation of rigid bodies. Computer Graphics 22(4): 299-308.

Hallquist, J.O. 1988. User's Manual for DYNA2D-An Explicit Two-Dimensional Hydrodynamic Finite Element Code with Interactive Rezoning and Graphical Display, UCID-18756, Rev. 3. Livermore, CA: LLNL.

Hardy, M.P., and S.J. Bauer. 1991. Drift Design Methodology and Preliminary Application for the Yucca Mountain Site Characterization Project. SAND89-0837. Albuquerque, NM: SNL.

Hardy, M.P., and S.J. Bauer. 1992. Rock mechanics considerations in designing a nuclear waste repository in hard rock. Proceedings of the 33rd U.S. Symposium on Rock Mechanics. J.R. Tillerson and W.R. Wawersik, eds. Rotterdam, Netherlands: A.A. Balkema: 1041-1050.

Harvie, C.E., N. Møller, and J.H. Weare. 1984. The prediction of mineral solubilities in natural waters: the $\mathrm{Na}-\mathrm{K}-\mathrm{Mg}-\mathrm{Ca}-\mathrm{Cl}-\mathrm{SO}_{4}-\mathrm{OH}-\mathrm{HCO}_{3}-\mathrm{CO}_{3}-\mathrm{CO}_{2}-\mathrm{H}_{2} \mathrm{O}$ system to high ionic strengths at $25 \mathrm{C}$. Geochimica et Cosmochimica Acta 48: 723-751.

Haworth, A., S.M. Sharland, P.W. Tasker, and C.J. Tweed. 1988a. A Guide to the Coupled Chemical Equilibria and Migration Code CHEQMATE. NSS-R.113. Oxon, United Kingdom: Harwell Laboratory.

Haworth, A., S.M. Sharland, P.W. Tasker, and C.J. Tweed. 1988b. Extensions to the Coupled Chemical Equilibria and Migration Code CHEQMATE. NSS-R.115. Oxon, United Kingdom: Harwell Laboratory.

Helgeson, H.C. 1970. A chemical and thermodynamic model of ore deposition in hydrothermal systems. Fiftieth Anniversary Symposia. B.A. Morgan, ed. Washington, DC: Mineralogical Society of America Special 3: 155-186.

Helgeson, H.C. 1979. Mass Transfer Among Minerals and Hydrothermal Solutions. H.L. Barnes, ed. New York, NY: Wiley.

Helgeson, H.C., and W.M. Murphy. 1983. Calculation of mass transfer as a function of time and surface area in geochemical processes. I. Computational approach. Mathematical Geology 15: 109-130.

Helgeson, H.C., J.M. Delany, H.W. Nesbitt, and D.K. Bird. 1978. Summary and critique of the thermodynamic properties of rock-forming minerals. American Journal of Science 278A: 1-229.

Helgeson, H.C., W.M. Murphy, and P. Aagaard. 1984. Thermodynamic and kinetic constraints on reaction rates among minerals and aqueous solutions. II. Rate constants, effective surface area, and the hydrolysis of feldspar. Geochimica et Cosmochimica Acta 48: 2405-2432.

Hem, J.D. 1985. Study and Interpretation of the Chemical Characteristics of Natural Water. Water-Supply Paper 2254. Alexandria, VA: USGS. 
Henley, R.W., A.H. Truescell, P.B. Barton, Jr., and J.A. Whitney. 1985. Fluid-Mineral Equilibria in Hydrothermal Systems: Reviews in Economic Geology, Volume 1. J.M. Robertson, ed. El Paso, TX: Society of Economic Geologists.

Hillel, D. 1980a. Fundamentals of Soil Physics. New York, NY: Academic Press.

Hillel, D. 1980b. Applications of Soil Physics. New York, NY: Academic Press.

Hodgkinson, D.P., and P.J. Bourke. 1980. Initial assessment of the thermal stresses around a radioactive waste depository in hard rock. Annals of Nuclear Energy 7: 541-552.

Hoek E., and Brown E.T. 1980. Underground Excavations in Rock. London, UK: IMM.

Hofmann, R. 1981a. STEALTH-A Lagrange Explicit Finite Difference Code for Solids, Structural and Thermohydraulic Analysis: Volume 1A: User's Manual-Theoretical Background and Numerical Equations. San Leandro, CA: Science Application, Inc.

Hofmann, R. 1981b. STEALTH-A Lagrangian Explicit Finite Difference Code for Solids, Structural, and Thermohydraulic Analysis, Introduction and Guide. EPRI-NP-2080-CCM-SY. Palo Alto, CA: EPRI.

Hohorst, J.K., A. Buccafurni, E.R. Carlson, R. Chambers, S.V. Chmielewski, D.L. Hagrman, N.L. Hampton, J.K. Hohorst, E.T. Laats, R.E. Mason, M.L. McComas, K.A. McNeil, R.L. Miller, M.A. Morgan, C.S. Olsen, and G.A. Reymann. 1990. SCDAP/RELAP5/MOD2 Code Manual, Volume 4: MATPRO-A Library of Materials Properties for Light-Water-Reactor Accident Analysis. NUREG/CR-5273. Washington, DC: NRC.

Holland, H.D., and S.D. Malinin. 1979. The solubility and occurrence of non-ore minerals. Geochemistry and Hydrothermal Ore Deposits. H.L. Barnes, ed. New York, NY: Wiley: 461-508.

Horton, R., P.J. Wierenga, and D.R. Nielsen. 1983. Evaluation of methods for determining the apparent thermal diffusivity of soil near the surface. Soil Science Society of America Journal 47(1): 25-32.

Horton, R., and P.J. Wierenga. 1984. The effect of column wetting on soil thermal conductivity. Soil Science 138(2): 101-108.

Hostetler, C.J., and R.L. Erikson. 1989. FASTCHEM ${ }^{T M}$ Package, Vol. 5: User's Guide to the EICM Coupled Geohydrochemical Transport Code. EPRI-EA-5870-CCM. Palo Alto, CA: EPRI.

Hostetler, C.J., R.L. Erikson, J.S. Fruchter, and C.T. Kincaid. 1989. FASTCHEM ${ }^{\text {TM }}$ Package, Vol. 1: Overview and Application to a Chemical Transport Problem. EPRI-EA-5870. Palo Alto, CA: EPRI.

Huyakorn, P.S., B.H. Lester, and J.W. Mercer. 1983. An efficient finite element technique for modeling transport in fractured porous media 2. Nuclide decay chain transport. Water Resources Research 19(5): 1286-1296. 
Incropera, F.P., and D.P. Dewitt. 1981. Fundamentals of Heat Transfer. New York, NY: Wiley.

Jackson, K.J. 1988. Verification and Validation Studies of the Addition of Pitzer's Equations to the EQ3/6 Brine Model. UCRL-53841. Livermore, CA: LLNL.

Jackson, K.J., and T.J. Wolery. 1985. Extension of the EQ3/6 Computer Codes to Geochemical Modeling of Brines. Scientific Basis for Nuclear Waste Management - VIII. C.M. Janzten, J.A. Stone, and R.C. Ewing, eds. Pittsburgh, PA: Materials Research Society: 507-514.

Jaeger, J.C., and N.G.W. Cook. 1979. Fundamentals of Rock Mechanics. Third Edition. London: Chapman and Hall.

Jamet, P., D. Fargue, and G. de Marsily. 1990. Coupled processes in the near-field. Proceedings From the Technical Workshop on Near-Field Performance Assessment for High-Level Waste Held in Madrid, October 15-17, 1990. P. Sellin, M. Apted, and J. Gago, eds. Stockholm, Sweden: SKB: 91-110.

Javandel, I., C. Doughty, and C. Tsang. 1984. Groundwater Transport: Handbook of Mathematical Models. Water Resources Monograph Series 10. Washington, DC: American Geophysical Union.

Jennings, A.A., and D.J. Kirkner. 1984. Instantaneous equilibrium approximation analysis. Journal of the Hydraulic Division of the American Society of Civil Engineers 110: 1700-1717.

Jing, L., J. Rutquist, O. Stephansson, C.F. Tsang, and F. Kautsky. 1993. DECOVALEX - Mathematical Models of Coupled H-T-M Processes for Nuclear Waste Repositories, Report of Phase I. Stockholm, Sweden: SKI.

Jinzhong, Y. 1988. Experimental and numerical studies of solute transport in two-dimensional saturated-unsaturated soil. Journal of Hydrology 97: 303-322.

Johnson, J.W., and D. Norton. 1991. Critical phenomena in hydrothermal systems: state, thermodynamic, electrostatic, and transport properties of $\mathrm{H}_{2} \mathrm{O}$ in the critical region. American Journal of Science 291: 541-648.

Johnson, J.W., E.H. Oelkers, and H.C. Helgeson. 1991. SUPCRT92: A Software Package for Calculating the Standard Molal Thermodynamic Properties of Minerals, Gases, Aqueous Species, and Reactions from 1 to 5000 bars and $0^{\circ}$ to $1000^{\circ} \mathrm{C}$. Livermore, CA: LLNL.

Jones, R.H. 1992. Stress-Corrosion Cracking. Materials Park, OH: ASM International.

Jones, R.H., and R.E. Ricker. 1992. Mechanics of stress-corrosion cracking. R.H. Jones, ed. Stress Corrosion Cracking. Materials Park, OH: ASM International: 1-40. 
Jury, W.A., H. El Abd, and T. Collins. 1983. Field scale transport of nonadsorbing and adsorbing chemicals applied to the soil surface. Proceedings of the National Water Well Association/U.S. Environmental Protection Agency Conference on Characterization and Monitoring of the Vadose (Unsaturated) Zone. D.M. Nielsen and M. Curl, eds. Dublin, OH: National Water Well Association.

Jury, W.A., G. Sposito, and R.E. White. 1986. A transfer function model of solute transport through soil. 1. Fundamental concepts. Water Resources Research 22(2): 243-247.

Kana, D.D., B.H.G. Brady, B.W. Vanzant, and P.K. Nair. 1991. Critical Assessment of Seismic and Geomechanics Literature Related to a High-Level Nuclear Waste Underground Repository. NUREG/CR-5440. Washington, DC: NRC.

Kelkar, S., and G.A. Zyvoloski. 1991. An efficient, three dimensional, fully-coupled hydro-thermomechanical simulation: FEHMS. 11th SPE Symposium on Reservoir Simulation. Anaheim, CA: SPE.

Kelsall, P.C., J.B. Case, and C.R. Chabannes. 1984. Evaluation of excavation-induced changes in rock permeability. International Journal of Rock Mechanics and Mineral Science and Geomechanical Abstracts 21(3): 123-135.

Kerrisk, J.F. 1987. Groundwater Chemistry at Yucca Mountain, Nevada, and Vicinity. LA-10929-MS. Los Alamos, NM: LANL.

Key, S.W. 1987. SPECTROM-331. A Finite Element Computer Program for the Large Deformation Elastic and Inelastic, Transient Dynamic Response of Three-Dimensional Solids and Structures. RE/SPEC Topical Report RSI-0299. Rapid City, SD: RE/SPEC.

Kincaid, C.T. 1988. FASTCHEM TM Package, Vol. 3: User's Guide to the ETUBE Pathline and Streamtube Database Code. EPRI-EA-5870-CCM. Palo Alto, CA: EPRI.

Kincaid, C.T., J.R. Morrey, and J.E. Rogers. 1984a. Geohydrochemical Models for Solute Migration, Vol I: Process Description and Computer Code Selection. EPRI-EA-3417. Palo Alto, CA: EPRI.

Kincaid, C.T., J.R. Morrey, S.B. Yabusaki, A.R. Felmy, and J.E. Rogers. 1984b. Geohydrochemical Models for Solute Migration, Vol. 2: Preliminary Evaluation of Selected Computer Codes. EPRI-EA-3417. Palo Alto, CA: EPRI.

Kipp, K.L. 1987. Effect of topography on gas flow in unsaturated fractured rock-Numerical simulation. D.D. Evans and T.J. Nicholson, eds. Flow and Transport through Unsaturated Fractured Rock, Geophysical Monograph 42. Washington, DC: AGU: 171-176.

Kirkner, D.J., and H. Reeves. 1988. Multicomponent mass transport with homogeneous and heterogeneous chemical reactions: Effect of the chemistry on the choice of numerical algorithm. 1. Theory. Water Resources Research 24(10): 1719-1729. 
Klavetter, E.A., and R.R. Peters. 1985. Development of Flow Models for Fractured, Porous Media Based on Two Different Conceptual Models. SAND85-0855C. Albuquerque, NM: SNL.

Klavetter, E.A., and R.R. Peters. 1986. Estimation of Hydrologic Properties of an Unsaturated, Fractured Rock Mass. SAND84-2642. Albuquerque, NM: SNL.

Kool, J.B., J.C. Parker, and M.T. Van Genuchten. 1987. Parameter estimation for unsaturated flow and transport models: a review. Journal of Hydrology 91: 255-293.

Krishnaswami, S., W.C. Graustein, K.K. Turekian, and J.F. Dowd. 1982. Radium, thorium and radioactive lead isotopes in groundwaters: application to the in situ determination of adsorption-desorption rate constants and retardation factors. Water Resources Research 18(6): 1633-1675.

Krupka, K.M., R.L. Erikson, S.V. Mattigod, J.A. Schramke, C.E. Cowan, L.E. Eary, J.R. Morrey, R.L. Schmidt, and J.M. Zachara. 1988. Thermochemical Data Used by the FASTCHEM ${ }^{T M}$ Package. EPRI-EA-5872. Palo Alto, CA: EPRI.

Kulacki, F.A., and M. Keyhani. 1987. Heat transfer aspects of nuclear waste disposal. Heat Transfer Problems in Nuclear Waste Management. E.V. McAssey, Jr., and V.E. Schrock, eds. HTD-67. New York, NY: ASME: 1-17.

Laryea, D.B., D.E. Elrick, and M.J.L. Robin. 1982. Hydrodynamic dispersion involving cationic adsorption during unsaturated transient water flow in soil. Soil Science of America Journal 46: 667-671.

Lasaga, A.C. 1981a. Rate laws of chemical reactions. Reviews in Mineralogy, Volume 8: Kinetics of Geochemical Processes. A.C. Lasaga, and R.J. Kirkpatrick, eds. Washington, DC: Mineralogical Society of America: 1-68.

Lasaga, A.C. 1981b. Transition State Theory. Reviews in Mineralogy, Volume 8: Kinetics of Geochemical Processes. A.C. Lasaga, and R.J. Kirkpatrick, eds. Washington, DC: Mineralogical Society of America: 135-170.

Lasaga, A.C. 1984. Chemical kinetics of water-rock interaction. Journal of Geophysical Research 89: 4009-4025.

Lasaga, A.C., and R.J. Kirkpatrick, eds. 1981. Reviews in Mineralogy, Volume 8: Kinetics of Geochemical Processes. Washington, DC: Mineralogical Society of America.

Lawn, B., and T. Wilshaw. 1975. Fracture of Brittle Solids. New York, NY: Cambridge University Press.

Leslie, B.W: 1991. Decay Series Disequilibrium Applied to the Study of Rock-Water Interaction in the Coso and the Salton Sea Geothermal Systems. Ph.D. Dissertation. Los Angeles, CA: University of Southem Califomia.

Lewis, T. 1990. Fluid flow in tectonic regimes. Short Course on Fluids in Tectonically Active Regimes of the Continental Crust. B.E. Nesbitt, ed. Mineralogical Association of Canada: 49-74. 
Lichtner, P.C. 1985. Continuum model for simultaneous chemical reactions and mass transport in hydrothermal systems. Geochimica et Cosmochimica Acta 49: 779-800.

Lichtner, P.C. 1988. The quasi-stationary state approximation to coupled mass transport and fluid-rock interaction in a porous medium. Geochimica et Cosmochimica Acta 52: 143-165.

Lichtner, P.C. 1992. Time-space continuum description of fluid-rock interaction in permeable media. Water Resources Research 28: 3135-3155.

Lichtner, P.C., and G.G. Biino. 1992. A first principles approach to supergene enrichment of a porphyry copper protore. I. Cu-Fe-S-H $\mathrm{H}_{2} \mathrm{O}$ subsystem. Geochimica et Cosmochimica Acta 56: 3987-4013.

Lichtner, P.C., and N. Waber. 1992. Redox front geochemistry and weathering: theory with application to the Osamu Utsumi uranium mine, Poços de Caldas, Brazil. Journal of Exploration Geochemistry 45: 521-564.

Lichtner, P.C., and V.N. Balashov. 1993. Metasomatic zoning: appearance of ghost zones in the limit of pure advective transport. Geochimica et Cosmochimica Acta 57: 369-387.

Lienhard, J.H. 1987. A Heat Transfer Textbook. Englewood Cliffs, NJ: Prentice-Hall.

Lin, W., and W.D. Daily. 1989. Laboratory study of fracture healing in Topopah Spring tuff-implications for near field hydrology. Nuclear Waste Isolation in the Unsaturated Zone Focus '89 Proceedings. La Grange Park, IL: ANS: 443-449.

Lin, W., and W. Daily. 1990. Hydrological properties of Topopah Spring tuff under a thermal gradient: laboratory results. International Journal of Rock Mechanics and Mineral Science and Geomechanical Abstracts 27: 373-385.

Lin, W., A. Ramirez, and D. Watwood. 1991. Temperature-Measurements from a Horizontal Heater Test in G-Tunnel. UCRL-JC-106693. Livermore, CA: LLNL.

Liu, C.W., and T.N. Narasimhan. 1989a. Redox-controlled multiple-species reactive chemical transport 1. model development. Water Resources Research 25(5): 869-882.

Liu, C.W., and T.N. Narasimhan. 1989b. Redox-controlled multiple-species reactive chemical transport 2. Verification and application. Water Resources Research 25(5): 883-910.

Love, A.E.H. 1944. A Treatise on the Mathematical Theory of Elasticity, 4th Edition. New York, NY: Dover.

Lu, N., S. Amter, and B. Ross. 1991. Effect of a low-permeability layer on calculated gas flow at Yucca Mountain. Proceedings of the International High-Level Radioactive Waste Management Conference. La Grange Park, II: ANS: 853-860.

Macdonald, D.D., and G.A. Cragnolino. 1989. Corrosion of steam cycle materials. The ASME Handbook on Water Technology for Thermal Power Systems. P. Cohen, ed. New York, NY: ASME. 
MacNeal, R.H. 1972. NASTRAN Theoretical Manual.Los Angeles, CA: MacNeal-Schwendler Corporation.

Mangold, D.C., and C. Tsang. 1991. A summary of subsurface hydrological and hydrochemical models. Reviews of Geophysics 29(1): 51-79.

Mansell, R.S., S.A. Bloom, H.M. Selim, and R.D. Rhue. 1988. Simulated transport of multiple cations in soil using variable selectivity coefficients. Soil Science Society of America Journal 52: 1533-1540.

Mansell, R.S., S.A. Bloom, and L.A.G. Aylmore. 1990. Simulating cation transport during unsteady, unsaturated water flow in sandy soil. Soil Science 150: 730-744.

Manteufel, R.D. 1991. Heat Transfer in an Enclosed Rod Array. Ph.D. Thesis. Cambridge, MA: MIT, Department of Mechanical Engineering.

Manteufel, R.D., R.T. Green, F.T. Dodge, and S.J. Svedeman. 1992. An experimental investigation of two-phase two-component nonisothermal flow in a porous medium with a simulated fracture. Heat and Mass Transfer in Porous Media. HTD-216. New York: ASME: 9-18.

McDowell-Boyer, L.M., J.R. Hunt, and N. Sitar. 1986. Particle transport through porous media. Water Resources Research 22(13); 1901-1921.

McKinley, I.G., and W.R. Alexander. 1992. Constraints on the applicability of in situ distribution coefficient values. Journal of Environmental Radiochemistry 15(1); 19-34.

Means, J.L., A.S. Maest, and D.A. Crerar. 1983. Organic Geochemistry of Deep Ground Waters and Radionuclide Partitioning Experiments Under Hydrothermal Conditions. ONWI-448. Columbus, $\mathrm{OH}$ : ONWI.

Miller, C.W. 1983. CHEMTRN User's Manual. LBL-16152. Berkeley, CA: Earth Sciences Division, LBL.

Miller, C.W., and L.V. Benson. 1983. Simulation of solute transport in a chemically reactive heterogeneous system: Model development and application. Water Resources Research 19(2); 381-391.

Moench, A.F., and A. Ogata. 1981. A numerical inversion of the Laplace transform solution to radial dispersion in a porous medium. Water Resources Research 17: 250-252.

Montazer, P., and W.E. Wilson. 1984. Conceptual Hydrologic Model of Flow in the Unsaturated Zone, Yucca Mountain, Nevada. Water Resources Investigations Report 84-4345 Denver, CO: USGS.

Moore, W.J. 1972. Physical Chemistry, 4th ed. Englewood Cliffs, NJ: Prentice-Hall.

Morland, L.W. 1974. Elastic response of regulatory jointed media. Geophysical Journal. Royal Astronomical Society 37; 435-446.

Morrey, J.R. 1988. FASTCHEM ${ }^{T M}$ Package. Volume 4: User's Guide to the ECHEM Equilibrium Geochemistry Code. EPRI-EA-5870-CCM. Palo Alto, CA: EPRI. 
Morrey, J.R., and C.J. Hostetler. 1985. Coupled geochemical and solute transport code development. Proceedings of the Conference on the Application of Geochemical Models to High-Level Nuclear Waste Repository Assessment, Oak Ridge, Tennessee, October 2-5, 1984. Jacobs, G.K., and S.K. Whatley, eds. NUREG/CP-0062. Washington, DC: NRC.

Morrey, J.R., C.T. Kincaid, C.J. Hostetler, S.B. Yabusaki, and L.W. Vail. 1986. Geohydrochemical Models for Solute Migration. Volume 3: Evaluation of Selected Computer Codes. EPRI-CCM EA-3417. Palo Aito, CA: EPRI.

Mulay, S.P. 1988. Diffusion of Heat and Mass Transfer in Partially Saturated Porous Media. Ph.D. Thesis. Chicago, IL: Illinois Institute of Technology.

Murphy, W.M. 1990. Performance assessment perspectives with reference to the proposed repository at Yucca Mountain, Nevada. Proceedings From the Technical Workshop on Near-Field Performance Assessment for High-Level Waste Held in Madrid, October 15-17, 1990. P. Sellin, M. Apted, and J. Gago, eds. Stockholm, Sweden: SKB: 11-24.

Murphy, W.M., and H.C. Helgeson. 1987. Thermodynamic \& kinetic constraints on reaction rates among minerals and aqueous solutions. III. Activated complexes \& $\mathrm{pH}$-dependence of the rates of feldspar, pyronene, wollastonite, and alivine hydrolysis. Geochimica et Cosmochimica Acta 51; 3137-3154.

Nagy, K.L., C.I. Steefel, A.E. Blume, and A.C. Lasaga. 1990. Dissolution and precipitation kinetics of kaolinite: Initial results at $80^{\circ} \mathrm{C}$ with application to porosity evolution in a sandstone. Prediction of Reservoir Quality Through Chemical Modeling. AAPG Memoir 49. I.D. Meshri and P.J. Ortoleva, eds. Tulsa, OK: American Association of Petroleum Geologists: 85-101.

Nair, S., D. Longwell, and C. Seigneur. 1990. Simulation of chemical transport in unsaturated soil. Journal of Environmental Engineering 116; 214-235.

Narasimhan, T.N., J.A. Apps, M. Zhu, and T.J. Vagele. 1992. Applications Handbook for FASTCHEM ${ }^{\mathrm{TM}}$ Volume 2: Chemical Transport Modules. EPRI-TR-101218, Volume 2. Palo Alto, CA: EPRI.

Nataraja, M.S., and T. Brandshaug. 1992. Staff Technical Position on Geologic Repository Operations Area Underground Facility Design-Thermal Loads. NUREG/1466. Washington, DC: NRC.

National Research Council. 1992. Ground Water at Yucca Mountain: How High Can it Rise? Washington, DC: National Academy Press.

Naumov, G.B., B.N. Ryzhenko, and I.L. Khodakovsky. 1974. Handbook of Thermodynamic Data. U.S. Geological Survey WRD-74-001. Springfield, VA: NTIS.

Neretnieks, I., and A. Rasmuson. 1984. An approach to modeling radionuclide migration in a medium with strongly varying velocity and block sizes along the flow path. Water Resources Research 20(12); 1823-1836. 
Nielsen, D.R., M.T. Van Genuchten, and J.W. Biggar. 1986. Water flow and solute transport processes in the unsaturated zone. Water Resources Research 22(9); 89s-108s.

Nilson, R.H., E.W. Peterson, K.H. Lie, N.R. Burkhard, and J.R. Hearst. 1991a. Atmospheric pumping: A mechanism causing vertical transport of contaminated gases through fractured permeable media. Journal of Geophysical Research 96(B13); 21,933-21,948.

Nilson, R.H., E.W. Peterson, K.H. Lie, N.R. Burkhard, and J.R. Hearst. 1991b. Barometric pumping of contaminated gases through fractured permeable media. Proceedings of the High-Level Radioactive Waste Management Conference. La Grange Park, IL: ANS: 861-868.

Nimick, F.B. 1990. The Thermal Conductivity of Seven ThermalMechanical Units at Yucca Mountain, Nevada. SAND88-1387. Albuquerque, NM: SNL.

Nitao, J.J. 1988. Numerical Modeling of the Thermal and Hydrological Environment Around a Nuclear Waste Package Using the Equivalent Continuum Approximation: Horizontal Emplacement. UCID21444. Livermore, CA: LLNL.

Nitao, J.J. 1989. V-TOUGH-An Enhanced Version of the TOUGH Code for the Thermal and Hydrologic Simulation of Large-Scale Problems in Nuclear Waste Isolation. UCID-21954. Livermore, CA: LLNL.

Nitao, J.J. 1991. Theory of matrix and fracture flow regimes in unsaturated, fractured porous media. Proceedings of the International High-Level Radioactive Waste Management Conference. La Grange Park, IL: ANS: 845-852.

Nitao, J.J., T.A. Buscheck, and D.A. Chesnut. 1992. The implications of episodic nonequilibrium fracturematrix flow on site suitability and total system performance. Proceedings of the International High-Level Radioactive Waste Management Conference. La Grange Park, IL: ANS: 279-296.

Nkedi-Kizza, P., J.W. Biggar, H.M. Selim, M.T. van Genuchten, P.J. Wierenga, and J.M. Davidson. 1984. On the equivalence of two conceptual models for describing ion exchange during transport through an aggregated oxisol. Water Resources Research 20(8); 1123-1130.

Noorishad, J., C.F. Tsang, and P.A. Witherspoon. 1984. Coupled thermal-hydraulic-mechanical phenomena in saturated fractured porous rocks: numerical approach. Journal Geophysical Research 89(B12); 10365-10374.

Noorishad, J., C.L. Carnahan, and L.V. Benson. 1987. Development of the Non-Equilibrium Reactive Chemical Transport Code CHMTRNS. LBL-22361. Berkeley, CA: LBL.

Noorishad, J., and C.F. Tsang. 1989. Recent Enhancement of the Coupled Hydro-Mechanical Code: ROCMAS II. Technical Report 89: 4. Stockholm, Sweden: SKI.

Nordstrom, D.K., and J.W. Ball. 1984. Chemical models, computer programs and metal complexation in natural waters. Complexation of Trace Metals in Natural Waters. C.J.M. Kramer and J.C. Duinker, ed. Netherlands: Martinus Nijhoff/Dr. J.W. Junk Publishing Co.: 149-169. 
Nordstrom, D.K., and J.L. Munoz. 1985. Geochemical Thermodynamics. Menlo Park, CA: The Benjamin/Cummings Publishing Co.

Nordstrom, D.K., L.N. Plummer, T.M.L. Wigley, T.J. Wolery, J.W. Ball, E.A. Jenne, R.L. Bassett, D.A. Crerar, T.M. Florence, B. Fritz, M. Hoffman, G.R. Holdren, G.M. Lafon, S.V. Mattigod, R.E. McDuff, F.M.M. Morel, M.M. Reddy, G. Sposito, and J. Thrailkill. 1979. Comparison of computerized chemical models for equilibrium calculations in aqueous systems. Chemical Modeling in Aqueous Systems. Speciation, Sorption, Solubility, and Kinetics. E.A. Jenne, ed. Washington, DC: ACS: 857-892.

Nordstrom, D.K., L.N. Plummer, D. Langmuir, E. Busenberg, H.M. May, B.F. Jones, and D.L. Parkhurst. 1990. Revised chemical equilibrium data for major water-mineral reactions and their limitations. Chemical Modeling of Aqueous Systems II. D.C. Melchior and R.L. Bassett, eds. Washington, DC: ACS: $398-413$.

Norton, D., and L.M. Cathles. 1979. Thermal aspects of ore deposition. Geochemistry of Hydrothermal Ore Deposits. H.L. Barnes, ed. New York, NY: Wiley: 611-631.

Nowacki, W. 1962. Thermoelasticity. Oxford, England: Pergamon Press.

O'Brien, G.M., and P. Tucci. 1992. Earthquake-induced water-level and fluid-pressure fluctuations at Yucca Mountain, Nevada. American Geophysical Union 1992 Fall Meeting. Abstract Supplement. Washington, DC: AGU.

Oelkers, E.H., and H.C. Helgeson. 1988. Calculation of the thermodynamic and transport properties of aqueous species at high pressures and temperatures: Aqueous tracer diffusion coefficients of ions to $1000^{\circ} \mathrm{C}$ and $5 \mathrm{~kb}$. Geochemica et Cosmochimica Acta 52: 63-85.

Oetting, F.L., M.H. Rand, and R.J. Ackerman. 1976. The Chemical Thermodynamics of Actinide Elements and Compounds. Part 1. The Actinide Elements. Vienna, Austria: IAEA.

Ohnishi, Y., M. Nishigaki, A. Kobayaski, and S. Akiyama. 1990. Three dimensional coupled thermohydraulic mechanical analysis code with PCG method. Proceedings of the International Symposium GEOVAL-90. Stockholm Sweden: SKI: 14-17.

Olague, N.E., D.E. Longsine, J.E. Campbell, and C.D. Leigh. 1991. User's Manual for the NEFTRAN II Computer Code. NUREG/CR-5618. Washington, DC: NRC.

Olsson, W.A., and A.K. Jones. 1980. Rock Mechanics Properties of Volcanic Tuffs from the Nevada Test Site. SAND80-1453. Albuquerque, NM: SNL.

O'Neal, W.C., D.W. Gregg, J.N. Hockman, E.W. Russell, and W. Stein. 1984. Preclosure Analysis of Conceptual Waste Package Designs for a Nuclear Waste Repository in Tuff. UCRL-53595. Livermore, CA: LLNL.

Ortoleva, P., E. Merino, J. Chadam, and C.H. Moore. 1987. Geochemical self-organization. I: Feedback mechanisms and modeling approach. American Journal of Science 287: 979-1002. 
Pabalan, R.T., and K.S. Pitzer. 1990. Chemical modeling of aqueous systems II. ACS Symposium Series II. Models for Aqueous Electrolyte Mixtures for Systems Extending from Dilute Solutions to Fused Salts. D. Melchior and R.L. Bassett, eds. Washington, DC: ACS: 44-57.

Pan, X.D., and M.B. Reed. 1991. A coupled distinct element-finite element method for large deformation analysis of rock masses. International Journal of Rock Mechanics and Mineral Science and Geomechanical Abstract 28(1): 93-99.

Papelis, C., K.F. Hayes, and J.O. Leckie. 1988. HYDRAQL: A Program for the Computation of Chemical Equilibrium Composition of Aqueous Batch Systems Including Surface-Complexation Modeling of Ion Adsorption at the Oxide/Solution Interface. Technical Report No. 306. Stanford, CA:Department of Civil Engineering, Stanford Univ.

Park, U.S. 1991. Discussion on coupled processes. SKB Technical Report 91-59. P. Sellin, M. Apted, and J. Gago, eds. Proceedings From the Technical Workshop on Near-Field Performance Assessment for High-Level Waste, Held in Madrid, October 15-17, 1990. Stockholm, Sweden: Swedish Nuclear Fuel and Waste Management Company: 108-110.

Parker, D.R., L.W. Zelazny, and T.B. Kinraide. 1987. Improvements to the program GEOCHEM. Soil Science Society of America Journal 51: 488-491.

Parker, J.C., and P.M. Jardine. 1986. Effects of heterogeneous adsorption behavior on ion transport. Water Resources Research 22(8): 1334-1340.

Parkhurst, D.L., D.C. Thorstenson, and L.N. Plummer. 1980. PHREEQE-A Computer Program for Geochemical Calculations. USGS Water Research Investigations 80-96. Reston, VA: USGS.

Parkhurst, D.L., D.C. Thorstenson, and L.N. Plummer. 1990. PHREEQE: A Computer Program for Geochemical Calculations: Revised and Reprinted August, 1990. USGS Water Research Investigations 80-96. Reston, VA: USGS.

Parry, W.T., J.M. Ballantyne, and N.L. Bryant. 1980. Hydrothermal alteration enthalpy and heat flow in the Roosevelt Hot Springs Thermal Area, UT. Journal of Geophysical Research 85: 2559-2566.

Patrick, W.C. et al. 1986. Spent Fuel Test-Climax: An Evaluation of the Technical Feasibility of Geologic Storage of Spent Nuclear Fuel in Granite. UCRL-53702. Livermore, CA: LLNL.

Peters, R.R., and E.A. Klavetter. 1988. A continuum model for water movement in an unsaturated fractured rock mass. Water Resources Research 24(3): 416-430.

Peters, R.R., E.A. Klavetter, I.J. Hall, S.C. Blair, P.R. Heller, and G.W. Gee. 1984. Fracture and Matrix Hydrologic Characteristics of Tuffaceous Materials from Yucca Mountain, Nye County, Nevada. SAND84-1471. Albuquerque, NM: SNL.

Philip, J.R., and D.A. de Vries. 1957. Moisture movement in porous materials under temperature gradients. Transactions, American Geophysical Union 38(2): 222-232. 
Phillips, S.L., F.V. Hale, L.F. Silvester, and M.D. Siegel. 1988. Thermodynamic Tables for Nuclear Waste Isolation: Aqueous Solutions Database. NUREG/CR-4864. Washington, DC: NRC.

Pinder, G.F., and W.G. Gray. 1977. Finite Element Simulation in Surface and Subsurface Hydrology. New York, NY: Academic Press.

Pitzer, K.S. 1973. Thermodynamics of electrolytes. 1: Theoretical basis and general equations. Journal of Physical Chemistry 77: 268-277.

Pitzer, K.S. 1979. Theory: ion interaction approach. Activity Coefficients in Electrolyte Solutions. M. Pytkowicz, ed. Boca Raton, FL: CRC Press: 157-208.

Plummer, L.N., B.F. Jones, and A.H. Truesdell. 1976. WATEQF-A FORTRAN IV Version of WATEQ, a Computer Program for Calculating Chemical Equilibrium of Natural Waters. Water Resources Investigations Report 76-13. Denver, CO: USGS.

Plummer, L.N., D.L. Parkhurst, G.W. Fleming, and S.A. Dunkle. 1988. A Computer Program Incorporating Pitzer's Equations for Calculation of Geochemical Reactions in Brines. Water Resources Investigations Report 88-4153. Washington, DC: USGS.

Pollock, D.W. 1982. Fluid Flow and Energy Transport in a High-Level Radioactive Waste Repository in Unsaturated Alluvium. Ph.D. Thesis. Urbana, IL: University of Illinois at Urbana-Champaign.

Pollock, D.W. 1986. Simulation of fluid flow and energy transport processes associated with high-level radioactive waste disposal in unsaturated alluvium. Water Resources Research 22(5): 765-775.

Potter, M.C., and J.F. Foss. 1982. Fluid Mechanics. Okemos, MI: Great Lakes Press.

Potter, R.W., and D.L. Brown. 1977. The Volumetric Properties of Aqueous Sodium Chloride Solutions From $0^{\circ}$ to $500^{\circ} \mathrm{C}$ and Pressures Up to 2000 bars Based on a Regression of Available Data in the Literature. USGS Bulletin 1421-C: Reston, VA: USGS.

Powers, D.W., J.W. Rudnicki, and L. Smith. 1991. External Peer Review Panel Majority Report. Washington, DC: DOE.

Price, J.G., S.T. Conlon, and C.D. Henry. 1987. Tectonic controls on orientation and size of epithermal veins. North American Conference on Tectonic Control of Ore Deposits. Rolla, MO: University of Missouri: 36-46.

Prigogine, I. 1955. Introduction to Thermodynamics of Irreversible Processes. New York, NY: Wiley.

Pruess, K. 1983. Development of the General Purpose Simulator MULKOM. Annual Report 1982. Berkeley, CA: LBL.

Pruess, K. 1985. A quantitative model of vapor dominated geothermal reservoirs as heat pipes in fractured porous rock. Transactions. Geothermal Resources Council 9(ii): 353-361. 
Pruess, K. 1987. TOUGH User's Guide. NUREG/CR-4645. Washington, DC: NRC.

Pruess, K., and J.S.Y. Wang. 1987. Numerical modeling of isothermal and nonisothermal flow in unsaturated fractured rock: A review. D.D. Evans and T.J. Nicholson, eds. Geophysical Monograph 42: Flow and Transport Through Unsaturated Fractured Rock. Washington, DC: American Geophysical Union: 11-21.

Pruess, K., J.S.Y. Wang, and Y.W. Tsang. 1987. Effective Continuum Approximation for Modeling Fluid and Heat Flow in Fractured Porous Tuff. SAND 86-7000. Albuquerque, NM: SNL.

Pruess, K., J.S.Y. Wang, and Y.W. Tsang. 1990a. On thermohydrologic conditions near high-level nuclear wastes emplaced in partially saturated fractured tuff. 1. Simulation studies with explicit consideration of fracture effects. Water Resources Research 26(6): 1235-1248.

Pruess, K., J.S.Y. Wang, and Y.W. Tsang. 1990b. On thermohydrologic conditions near high-level nuclear wastes emplaced in partially saturated fractured tuff. 2. Effective continuum approximation. Water Resources Research 26(6): 1249-1261.

Pusch, T. 1989. Alteration of the hydraulic conductivity of rock by tunnel excavation. International Journal of Rock Mechanics and Mineral Science and Geomechanical Abstracts 26(1): 79-83.

Radhakrishna, H.S., K.C. Lau, and A.M. Crawford. 1990. Experimental modeling of the near-field thermal regime in a nuclear-fuel waste disposal vault. Engineering Geology 28: 337-351.

Rajen, G., and F.A. Kulacki. 1987. Experimental and numerical study of natural convection in a porous layer locally heated from below - A regional laboratory model for a nuclear waste repository. HTD-67. E.V. McAssey, Jr., and V.E. Schrock, eds. Heat Transfer Problems in Nuclear Waste Management. New York, NY: ASME: 19-26.

Ramirez, A.L. (ed), T. Buscheck, R. Carlson, W. Daily, K. Lee, W. Lin, N. Mao, A. Ramirez, T. Ueng, H. Wang, and D. Watwood. 1991a. Prototype Engineered Barrier Systems Field Test (PEBSFT) Final Report. UCID-106159. Livermore, CA: LLNL.

Ramirez, A.L., R.C. Carlson, and T.A. Buscheck. 1991b. In Situ Changes in the Moisture Content of Heated, Welded Tuff Based on Thermal Neutron Measurements. UCRL-ID-104715. Livermore, CA: LLNL.

Ramspott, L.D. 1991. The constructive use of heat in an unsaturated tuff repository. Proceedings of the International High-Level Radioactive Waste Management Conference. La Grange Park, IL: ANS: $1602-1607$.

Rard, J.A. 1989. Chemical Thermodynamics of Technetium. UCRL-100554. Livermore, CA: LLNL.

Rasmussen, T.C., and D.D. Evans. 1987. Unsaturated Flow and Transport Through Fractured Rock Related to High-Level Waste Repositories. NUREG/CR-4655. Washington, DC: NRC. 
Rasmussen, T.C., and D.D. Evans. 1989. Fluid Flow and Solute Transport Modeling Through ThreeDimensional Networks of Variably Saturated Discrete Fractures. NUREG/CR-5239. Washington, DC: NRC.

Rasmussen, T.C., D.D. Evans, P.J. Sheets, and J.H. Blanford. 1990. Unsaturated Fractured Rock Characterization Methods and Data Sets at the Apache Leap Tuff Site. NUREG/CR-5596. Washington, DC: NRC.

Read, D., and T.W. Broyd. 1991. Recent progress in testing chemical equilibrium models: The CHEMVAL project. Radiochim Acta 52/53: 453-456.

Reeves, H., and D.J. Kirkner. 1988. Multicomponent mass transport with homogeneous and heterogeneous chemical reactions: effect of the chemistry on the choice of numerical algorithm. 2 . Numerical results. Water Resources Research 24(10): 1730-1739.

Richardson, S.M., and H.Y. McSween. 1989. Geochemistry: Pathways and Processes. Englewood Cliffs, NJ: Prentice Hall.

Rimstidt, J.D., W.D. Newcomb, and D.L. Shettel, Jr. 1989. A vertical thermal gradient experiment to simulate conditions in vapor dominated geothermal systems, epithermal gold deposits, and highlevel radioactive repositories in unsatirated media. Proceedings of the 4th International Symposium on Water-Rock Interaction. A.A. Balkema, in Rotterdam, Netherlands: 585-588.

Robie, R.A., B.S. Hemingway, and J.R. Fisher. 1978. Thermodynamic Properties of Minerals and Related Substances at $298.15 \mathrm{~K}$ and $1 \mathrm{Bar}\left(10^{5}\right.$ Pascals) Pressure and at Higher Temperatures. USGS Bull. 1452, reprinted with corrections 1979. Washington, DC: USGS.

Rockhold, M.L., B. Sagar, and M.P. Connelly. 1992. Three-Dimensional Modeling of Unsaturated Flow in the Vicinity of Proposed Exploratory Shaft Facilities at Yucca Mountain, Nevada. PNL-7474. Richland, WA: PNL.

Roedder, E. 1984. Reviews in Mineralogy, Volume 12: Fluid Inclusions. Washington, DC: Mineralogical Society of America.

Roglans-Ribas, J., and B.I. Spinrad. 1989. A simplified thermal analysis of a nuclear waste repository. Annals of Nuclear Energy 16(8): 371-382.

Rose, A.W., and D.M. Burt. 1979. Hydrothermal alteration. Geochemistry of Hydrothermal Ore Deposits. H.L. Barnes, ed. New York, NY: Wiley: 173-235.

Ross, B. 1992. Temperature scenarios for a repository at Yucca Mountain. Proceedings of the International High-Level Radioactive Waste Management Conference. La Grange Park, IL: ANS: 784-789.

Roth, K., W.A. Jury, and H.A. Fluhler. 1990a. Solute transport through unsaturated soil: the field evidence. Agronomy Abstracts, Soil Science Society of America 82nd Annual Meeting. San Antonio, TX: Soil Science Society of America: 218. 
Roth, K., H. Fluhler, W.A. Jury, and J.C. Parker. 1990b. Prediction of cation transport in soils using cation exchange reactions. Field-Scale Solute and Water Transport Through Soil. H.M. Selim, R.S. Mansell, L.A. Gaston, H. Fluhler, and R. Schulin, eds. Basel, Switzerland: Birkhauser Verlag: 223-238.

Rubin, J. 1983. Transport of reacting solutes in porous media: relation between mathematical nature of problem formulation and chemical nature of reactions. Water Resources Research 19(5): 1231-1252.

Rubin, J., and R.V. James. 1973. Dispersion affected transport of reacting solutes in saturated porous media: Galerkin method applied to equilibrium-controlled exchange in unidirectional steady water flow. Water Resources Research 9(5): 1332-1356.

Runchal, A.K., and B. Sagar. 1992. PORFLOW: A Multifluid Multiphase Model for Simulating Flow, Heat Transfer and Mass Transport in Fractured Porous Media, User's Manual - Version 2.40. CNWRA 92-003. San Antonio, TX: CNWRA.

Russo, D. 1989a. Field-scale transport of interacting solutes through the unsaturated zone: 1 . Analysis of the spatial variability of the transport properties. Water Resources Research 25(12): 2475-2485.

Russo, D. 1989b. Field scale transport of interacting solutes through the unsaturated zone: 2 . Analysis of the spatial variability of the field response. Water Resources Research 25(12): 2487-2495.

Rutqvist, J., O. Stephansson, J. Noorishad, and C.F. Tsang. 1991. Modeling of hydro-thermo-mechanical effects in a fracture intersecting a nuclear waste deposition hole. Proceedings of the International High-Level Radioactive Waste Management Conference. La Grange Park, IL: ANS: 547-554.

Sadiq, M., and W.L. Lindsay. 1979. Selection of Standard Free Energies of Formation for Use in Soil Chemistry. Technical Bulletin 134. Fort Collins, CO: CSUES.

Sadiq, M., and W.L. Lindsay. 1981. Arsenic Supplement to Technical Bulletin 134: Selection of Standard Free Energies of Formation for Use in Soil Chemistry. Technical Bulletin 134 (supplement). Fort Collins, CO: CSUES.

Sagar, B. 1991. PORMC: A Model for Monte Carlo Simulation of Fluid Flow, Heat and Mass Transport in Variably Saturated Geologic Media. WHC-EP-0445. Richland, WA: Westinghouse Hanford Co.

Sagar, B., and A.K. Runchal. 1990. PORFLO-3: A Mathematical Model for Fluid Flow, Heat, and Mass Transport in Variably Saturated Geologic Media, Theory and Numerical Methods, Version 1.0. WHC-EP-0042. Richland, WA: Westinghouse Hanford Company.

Sagar, B., and A.B. Gureghian. 1991. Performance Assessment Research. Report on Research Activities for Calendar Year 1990. W.C. Patrick. CNWRA 90-01A. San Antonio, TX: CNWRA.

Sagar, B., G.W. Wittmeyer, and A.C. Bagtzoglou. 1992. Chapter 9 - Performance Assessment Research. Report on Research Activities for Calendar Year 1991. W.C. Patrick, ed. CNWRA 91-01A. San Antonio, TX: CNWRA. 
Schubert, G., and J.M. Straus. 1979. Steam-water counterflow in porous media. Journal of Geophysical Research 84(B4): 1621-1628.

Scott, R.B., R.W. Spengler, S. Diehl, A.R. Lappin, and M.P. Chornack. 1982. Geologic character of tuffs in the unsaturated zone at Yucca Mountain, southern Nevada. Role of the Unsaturated Zone in Radionuclide; and Hazardous Waste Disposal. J. Mercer, P.S. Rao, and I.W. Marine, eds. Ann Arbor, MI: Ann Arbor Science: 289-335.

Selim, H.M., J.M. Davidson, and R.S. Mansell. 1976. Evaluation of a 2-site adsorption-desorption model for describing solute transport in soils. 1976 Summer Computer Simulation Conference. La Jolla, CA: Simulation Councils, Inc.: 444-448.

Selim, H.M., R.S. Mansell, L.A. Gaston, H. Fluhler, and R. Schulin. 1990. Field-scale solute and water transport through soil. Prediction of Cation Transport in Soils Using Cation Exchange Reactions. K. Roth, H. Fluhler, W.A. Jury, and J.C. Parker, eds. Basel, Switzerland: Birkhauser Verlag: 223-238.

Serne, R.J., R.C. Arthur, and K.M. Krupka. 1990. Review of Geochemical Processes and Codes for Assessment of Radionuclide Migration Potential at Commercial LLW Sites. PNL-7285. Richland, WA: PNL.

Shen, B., and O. Stephansson. 1990a. 3DEC: Mechanical and Thermomechanical Analysis of Glaciation and Thermal Loading of a Waste Repository. SKITR90: 3. Stockholm, Sweden: SKI.

Shen, B., and O. Stephansson 1990b. Modeling of Rock Mass Response to Repository Excavations, Thermal Loading from Radioactive Waste and Welling Pressure of Buffer Material. SKITR90: 12. Stockholm, Sweden: SKI.

Shi, G.H., and R.E. Goodman 1988. Discontinuous Deformation Analysis - A New Method for Computing Stress, Strain and Sliding of Block Systems. 29th U.S. Symposium on Rock Mechanics. Rotterdam, Netherlands: A.A. Balkema, 381-393.

Shock, E.L., and H.C. Helgeson. 1988. Calculation of the thermodynamic and transport properties of aqueous species at high pressures and temperatures: correlation algorithms for ionic species and equation of state predictions to $5 \mathrm{~kb}$ and $1000^{\circ} \mathrm{C}$. Geochimica et Cosmochimica Acta 52: 20092036.

Shock, E.L., and H.C. Helgeson. 1990. Calculation of the thermodynamic and transport properties of aqueous species at high pressures and temperatures: standard partial molal properties of organic species. Geochimica ẹt Cosmochimica Acta 54: 915-945.

Shreir, L.L. 1976. Corrosion. Volume 1: Metal-Environment Reactions. London: Newnes-Butterworths. 
Siegel, M.D., R. Rechard, K.L. Erickson, J.O. Leckie, D.B. Kent, D.A. Grover, S.J. Phillips, R.V. Guzowski, and S. Faith. 1989. Progress in Development of a Methodology for Geochemical Sensitivity Analysis for Performance Assessment. Volume 2: Speciation, Sorption, and Transport in Fractured Media. NUREG/CR-5085. Washington, DC: NRC.

Siegel, R., and J.R. Howell. 1992. Thermal Radiation Heat Transfer. Washington, DC: Taylor and Francis.

Siyag, R.S., R. Pal, and S.R. Poonia. 1983. Water transmission of unsaturated soil samples in relation to mixed $\mathrm{Na}-(\mathrm{Ca}+\mathrm{Mg})$ solutions. Geoderma 31 : 107-116.

Skopp, J., W.R. Gardner, and E.J. Tyler. 1981. Solute movement in structured soils: two-region model with small interaction. Soil Science Society of America Journal 45: 837-842.

Smith, R.M., and A.E. Martell. 1976. Critical Stability Constants. Volume 4: Inorganic Complexes. New York, NY: Plenum Press.

Sokolnikoff, I.S. 1956. Mathematical Theory of Elasticity. New York, NY: McGraw-Hill.

Sourirajan, S., and G.C. Kennedy. 1962. The system $\mathrm{NaCl}-\mathrm{H}_{2} \mathrm{O}$ at elevated temperatures and pressures. American Journal of Science 260: 114-141.

Sposito, G. 1986. Corrections to the program GEOCHEM, Soil Science Society of America Journal 50: 270.

Sposito, G., and S.V. Mattigod. 1980. GEOCHEM: A Computer Program for the Calculation of Chemical Equilibria in Soil Solutions and Other Natural Water Systems. Riverside, CA: University of California, Riverside.

Sposito, G., R.E. White, P.R. Darrah, and W.A. Jury. 1986. A transfer function model of solute transport through soil. 3. The convection-dispersion equation. Water Resources Research 22(2): 255-262.

Steefel, C.I., and A.C. Lasaga. 1990. The evolution of dissolution patterns: permeability change due to coupled flow and reaction. Chemical Modeling of Aqueous Systems II. D.C. Melchior and R.L. Bassett, eds., Washington, DC: ACS: 212-225.

Sterner, S.M., and R.J. Bodnar. 1991. Synthetic fluid inclusions. X: experimental determination of $\mathrm{P}-\mathrm{V}-\mathrm{T}-\mathrm{X}$ properties in the $\mathrm{CO}_{2}-\mathrm{H}_{2} \mathrm{O}$ system to $6 \mathrm{~Kb}$ and $700^{\circ} \mathrm{C}$. American Joumal of Science 291: 1-54.

Stumm, W., and J.J. Morgan. 1981. Aquatic Chemistry. An Introduction Emphasizing Chemical Equilibria in Natural Waters. New York, NY: Wiley Interscience.

Suarez, D.L., J.D. Rhoades, R. Lavado, and C.M. Grieve. 1984. Effect of pH on saturated hydraulic conductivity and soil dispersion. Soil Science Society of America Journal. 48: 50-55. 
Takenouchi, S., and G.C. Kennedy. 1964. The binary system $\mathrm{H}_{2} \mathrm{O}-\mathrm{CO}_{2}$ at high temperatures and pressures. American Journal of Science 262: 1055-1074.

Takenouchi, S., and G.C. Kennedy. 1965. The solubility of carbon dioxide in $\mathrm{NaCl}$ solutions at high temperatures and pressures. American Joumal of Science 263: 445-454.

Tanger, J.C. IV, and H.C. Helgeson. 1988. Calculation of the thermodynamic and transport properties of aqueous species at high pressures and temperatures: revised equations of state for the standard partial molal properties of ions and electrolytes. American Joumal of Science 288: 19-98.

Thomas, D.M., and Gudmundsson. 1989. Advances in the study of solids deposition in geothermal systems. Geothermics 18: 5-15.

Thomson, W. 1871. On the equilibrium of vapor at a curved surface of liquid. Philosophical Magazine 42: $448-452$.

Thorstenson, D.C., E.P. Weeks, H. Haas, and J.C. Woodward. 1989. Physical and chemical characteristics of topographically affected airflow in an open borehole at Yucca Mountain, Nevada. Proceedings: Nuclear Waste Isolation in the Unsaturated Zone, Focus '89. La Grange Park, IL: ANS: 256-270.

Timoshenko, S.P., and J.N. Goodier. 1970. Theory of Elasticity. New York, NY: McGraw-Hill.

Todreas, N.E., and M.S. Kazimi. 1990. Nuclear Systems I, Thermal Hydraulic Fundamentals. New York, NY: Hemisphere.

Tompson, A.F.B., and L.W. Gelhar. 1990. Numerical simulation of solute transport in three-dimensional, randomly heterogeneous porous media. Water Resources Research 26: 2541-2562.

Tompson, A.F.B., and R.B. Knapp. 1991. Large-scale simulation of solute transport in heterogeneous and reactive porous media. L.W. Younker, ed., Earth Sciences Report 1989/90. UCRL-LR-106135. Livermore, CA: LLNL: 89-102.

Tompson, A.F.B., and D.E. Dougherty. 1992a. Highly resolved simulations of chemical migration in physically and chemically heterogeneous porous media. T.F. Russell et al., eds. Computational Methods in Water Resources IX. Vol. I: Numerical Methods in Water Resources. Boston, MA: Computational Mechanics Publications: 671-686.

Tompson, A.F.B., and D.E. Dougherty. 1992b. Particle-grid methods for reacting flows in porous media with application to Fisher's equation. Applied Mathematical Modelling 16: 374-383.

Tompson, A.F.B., and R.B. Knapp. 1989. Reactive Chemical Transport Problems in Nuclear Waste Analyses. UCRL-99552. Livermore, CA: LLNL.

Travis, B.J. 1984. TRACR3D: A Model of Flow and Transport in Porous/Fractured Media. LA-9667-MS. Los Alamos, NM: LANL. 
Travis, B.J., and H.E. Nuttall. 1987. Two-Dimensional Numerical Simulation of Geochemical Transport in Yucca Mountain. LA-10532-MS. Los Alamos, NM: LANL.

Tripathi, V.S. 1984. Uranium(VI) Transport Modeling: Geochemical Data and Submodels. Ph.D. Dissertation. Stanford, CA: University of Stanford.

Tritton, D.J. 1977. Physical Fluid Dynamics. Berkshire, England, United Kingdom: Von Nostrand Reinhold.

Truesdell, A.H., and B.F. Jones. 1974. WATEQ—A computer program for calculating chemical equilibria of natural water. Journal of Research, U.S. Geological Survey 2: 233-248.

Tsang, C.F. 1987a. Introduction to coupled processes. Coupled Processes Associated with Nuclear Waste Repositories. C.F. Tsang, ed. San Diego, CA: Academic Press: 1-6.

Tsang, C.F. 1987b. Some considerations in the validation of coupled hydrothermal and hydrothermomechanical models. GEOVAL 1987. Stockholm, Sweden: SKI: 507-526.

Tsang, C.F. 1991. Coupled hydromechanical-thermochemical processes in rock fractures. Reviews of Geophysics 29(4): 537-551.

Tsang, Y.W., and K. Pruess. 1987. A study of thermally induced convection near a high-level waste repository in partially saturated fractured tuff. Water Resources Research 23(10): 1958-1966.

Turcotte, D.L. 1989. A heat pipe mechanism for volcanism and tectonics on Venus. Journal of Geophysical Research 94(B3): 2779-2785.

Tumer, D.R. 1991a Effects of Variable Hydrologic Saturation on Sorption Modeling for High-Level Waste Assessment: A Literature Review. CNWRA 91-016. San Antonio, TX: CNWRA.

Tumer, D.R. 1991b. Sorption Modeling for High-Level Waste Performance Assessment: A Literature Review. CNWRA 91-011. San Antonio, TX: CNWRA.

Tumer, D.R., T. Griffin, and T. Dietrich. 1993. Radionuclide sorption modeling using the MINTEQA2 speciation code. Scientific Basis for Nuclear Waste Management-XVI. Pittsburgh, PA: MRS: 783790.

Tweed, C.J. 1988. A Guide to PICKER-A Data-Selection Tool for the Program PHREEQE. AERE-R.12515. Oxon, United Kingdom: Harwell Laboratory.

Ungs, M.J., K.V. Summers, and S.A. Gherini. 1986. MYGRT: An IBM Personal Computer Code for Simulating Solute Migration in Groundwater User's Manual. EPRI-EA-4543-CCM. Palo Alto, CA: EPRI.

Updegraff, C.D. 1989. Comparison of Strongly Heat-Driven Flow Codes for Unsaturated Media. NUREG/CR-5367. Washington, DC: NRC. 
Valocchi, A.J. 1985. Validity of the local equilibrium assumption for modeling sorbing solute transport through homogeneous soils. Water Resources Research 21(6): 808-820.

Valocchi, A.J., and M. Malmstead. 1992. Accuracy of operator splitting for advection-diffusion-reaction problems. Water Resources Research 28(5): 1471-1476.

Valocchi, A.J., P.V. Roberts, G.A. Parks, and R.L. Street. 1981a Simulation of the transport of ion-exchanging solutes using laboratory-determined chemical parameter values. Ground Water 19(6): 600-607.

Valocchi, A.J., R.L. Street, and P.V. Roberts. 1981b. Transport of ion-exchanging solutes in groundwater: chromatographic theory and field simulation. Water Resources Research 17(5): 1517-1527.

van der Zee, S.E.A.T.M. 1990a. Analysis of solute redistribution in a heterogeneous field. Water Resources Research 26(2): 273-278.

van der Zee, S.E.A.T.M. 1990b. Analytical traveling wave solutions for transport with nonlinear and nonequilibrium adsorption. Water Resources Research 26: 2563-2578.

van der Zee, S.E.A.T.M., and W.H. van Riemsdijk. 1987. Transport of reactive solute in spatially variable soil systems. Water Resources Research 23: 2059-2069.

van der Zee, S.E.A.T.M., and J.J.T.I. Boesten. 1991. Effects of soil heterogeneity on pesticide leaching to groundwater. Water Resources Research 27(12): 3051-3063.

van der Zee, S.E.A.T.M., and G.H. Bolt. 1991. Deterministic and stochastic modeling of reactive solute transport. Journal of Contaminant Hydrology 7: 75-93.

van Eijkeren, J.C.H., and J.P.G. Loch. 1984. Transport of cationic solutes in sorbing porous media. Water Resources Research 20(6): 714-718.

van Genuchten, R. 1978. Calculating the Unsaturated Hydraulic Conductivity with a New, Closed-Form Analytical Model. Research Report 78-WR-08. Princeton, NJ: Department of Civil Engineering, Princeton University.

van Genuchten, M.T. 1980. A closed-form equation for predicting the hydraulic conductivity of unsaturated soils. Soil Science Society of America Joumal 44: 892-898.

van Genuchten, M.T., and PJ. Wierenga. 1976. Mass transfer studies in sorbing porous media, 1. Analytical solutions. Soil Science Society of America Joumal 40: 473-480.

van Genuchten, M.T., and W.J. Alves. 1982. Analytical Solutions of the One-Dimensional ConvectiveDispersive Solute Transport Equation. U.S. Department of Agriculture Bull. 1661. Washington, DC: USDA.

van Genuchten, M.T., and W.A. Jury. 1987. Progress in unsaturated flow and transport modeling. Reviews of Geophysics 25(2): 135-140. 
van Konynenburg, R.A. 1991. Gaseous release of carbon-14: why the high-level waste regulations should be changed. Proceedings of the International High-Level Radioactive Waste Management Conference. La Grange Park, IL: ANS: 313-319.

Vaughan, P.J. 1987. Analysis of permeability reduction during flow of heated, aqueous fluid through Westerly Granite. Coupled Processes Associated with Nuclear Waste Repositories. C. Tsang, ed. Orlando, FL: Academic Press: 529-539.

Verma, K., and K. Pruess. 1988. Thermohydrological conditions and silica redistribution near high-level nuclear waste emplaced in saturated geological formations. Journal of Geophysical Research 93(B2): 1159-1173.

Viani, B. 1988. Interim Report on Modeling Sorption with EQ3/6. UCID-21308. Livermore, CA: LLNL.

Voegele, M., E. Gardin, D. Lingle, M. Board, and N. Barton. 1981. Site characterization of joint permeability using the heated block test. 22nd U.S. Symposium on Rock Mechanics. Cambridge, MA: MIT: $120-127$.

Vutukuri, V. 1974. The effect of liquids on the tensile strength of limestone. International Joumal of Rock Mechanics 11: 27-29.

Wada, C., and H. Takahashi. 1990. Prediction of changes in elastic moduli due to water-rock interaction during operation of granitic HDR geothermal system. Geothermics 19(N1): 61-75.

Wagman, D.D., W.H. Evans, V.B. Parker, R.H. Shumm, I. Halow, S.M. Bailey, K.L. Chumey, and R.L. Nuttall. 1982. The NBS Tables of Chemical Thermodynamic Properties. Selected Values for Inorganic and C1 and C2 Organic Substances in SI Units. New York, NY: ACS.

Walsh, M.P., L.W. Lake, and R.S. Schechter. 1982. A description of chemical precipitation mechanisms and their role in formation damage during stimulation by hydrofluoric acid. Journal of Petroleum Technology 34: 2097-2112.

Walsh, M.P., S.L. Bryant, R.S. Schechter, and L.W. Lake. 1984. Precipitation and dissolution of solids attending flow through porous media. American Institute of Chemical Engineering Journal. 30: 317-327.

Walton, F.B., J.P.M. Ross, and D.G. Juhnke. 1985. The effects of simultaneous heat and mass transport on radionuclide migration. Scientific Basis for Nuclear Waste Management - VIII. C.M. Jantzen, J.A. Stone, and R.C. Ewing, eds. Pittsburgh, PA: MRS: 663-672.

Wang, J.S.Y. 1992. Variations of hydrological parameters of tuff and soil. Proceedings of the International High-Level Radioactive Waste Management Conference. La Grange Park, IL: ANS: 727-736.

Wang, J.S.Y., and C.F. Tsang. 1980. Buoyancy flow in fractures intersecting a nuclear waste repository. Heat Transfer in Nuclear Waste Disposal. F.A. Kulacki, and R.W. Lyczkowski, eds. HTD-16. Chicago, II: ASME: 105-112. 
Wang, J.S.Y., D.C. Mangold, R.K. Spencer, and C.F. Tsang. 1983. Thermal Impact of Waste Emplacement and Surface Cooling Associated with Geologic Disposal of Nuclear Waste. NUREG/CR-2910. Washington, DC: NRC.

Wang, J.S.Y., and T.N. Narasimhan. 1985. Hydrologic mechanisms governing fluid flow in a partially saturated, fractured, porous medium. Water Resources Research 21(12): 1861-1874.

Wang, J.S.Y., and T.N. Narasimhan. 1986. Hydrologic Mechanisms Governing Partially Saturated Fluid Flow in Fractured Welded Units and Porous Nonwelded Units at Yucca Mountain. LBL-21022. Berkeley, CA: LBL.

Wang, H.F., B.P. Bonner, S.R. Carlson, B.J. Kowallis, and H.C. Heard. 1989. Thermal stress cracking in granite. Journal of Geophysical Research 94(B2): 1745-1758.

Wark, K. 1983. Thermodynamics. New York, NY: McGraw-Hill.

Weeks, E.P. 1987. Effect of topography on gas flow in unsaturated fractured rock-Concepts and observations. D.D. Evans and T.J. Nicholson, eds. Flow and Transport through Unsaturated Fractured Rock, Geophysical Monograph 42. Washington, DC: AGU: 165-170.

Westall, J. 1979. MICROQL: I. A Chemical Equilibrium Program in BASIC. Zurich, Switzerland: Swiss Federal Institute of Technology.

Westall, J.C., J.L. Zachary, and F.M.M. Morel. 1976. MINEQL, A Computer Program for the Calculation of Chemical Equilibrium Composition of Aqueous Systems. Tech. Note 18. Cambridge, MA: MTT.

Westwood, A. 1974. Control and application of environment sensitive fracture processes. Journal of Material Science 9: 1871-1895.

Whirley, R.G. 1991: DYNA3D_A Nonlinear, Explicit, Three-Dimensional Finite Element Code for Solid and Structural Mechanics-User Manual, UCRL-MA107254. Livermore, CA: LLNL.

Whitaker, S. 1977. Simultaneous heat, mass, and momentum transfer in porous media: theory of drying. Advances in Heat Transfer 13: 119-203.

White, M.D., and M.K. Altenhofen. 1989. A sensitivity study of near-field thermal and hydrological conditions in tuff. Proceedings of the Topical Meeting on Nuclear Waste Isolation in the Unsaturated Zone, FOCUS '89. La Grange Park, IL: ANS: 20-29.

Wijesinghe, A.M. 1989. Hydrothermomechanical simulator development task Repository Technology Program Activities: FY 1988. J. Yow, Jr. et al., eds. UCID-21600. Berkeley, CA: LLNL: 3-14.

Williams, J.R., G. Hocking, and G.G.W. Mustoe 1985. The theoretical basis of the discrete element method. Proceedings of the NUMETA '85 Conference. Swansea, UK: A. A. Balkema: 897-906.

Wilson, M.L., and A.L. Dudley. 1986. Radionuclide Transport in an Unsaturated, Fractured Medium. SAND-86-7017C. Albuquerque, NM: SNL. 
Witherspoon, P.A., J.S.Y. Wang, K. Iwai, and J.E. Gale. 1979. Validity of cubic law for fluid flow in a deformable rock fracture. Water Resources Research 16(6): 1016-1024.

Wolery, T.J. 1979. Calculation of Chemical Equilibrium Between Aqueous Solution and Minerals. The EQ3/6 Software Package. UCRL-52658. Berkeley, CA: LLNL.

Wolery, T.J. 1983. EQ3NR. A Computer Program for Geochemical Aqueous Speciation-Solubility Calculations: User's Guide and Documentation. UCRL-53414. Livermore, CA: LLNL.

Wolery, T.J., K.J. Jackson, W.L. Bourcier, B.E. Bruton, K.G. Knauss, and J.M. Delany. 1990. Current status of the EQ3/6 software package for geochemical modeling. Chemical Modeling of Aqueous Systems II. D. Melchior and R. L. Bassett, eds. ACS Symposium Series 416. Washington, DC: SCS: $104-116$.

Wollenberg, H.A., J.S.Y. Yang, and G. Korbin. 1983. An Appraisal of Nuclear Waste Isolation in the Vadose Zone in Arid and Semi-Arid Regions. NUREG/CR 3158. Washington, DC: NRC.

Woods, T.L., and R.M. Garrels. 1987. Thermadynamic Values at Low Temperature for Natural Inorganic Materials: An Uncritical Summary. New York, NY: Oxford University Press.

Yeh, G.T. 1985. Comparisons of successive iteration and direct methods to solve finite element equations of aquifer contaminant transport. Water Resources Research 21(3): 272-280.

Yeh, G.T. 1987. FEMWATER: A Finite Element Model of Water Flow Through Saturated-Unsaturated Porous Media, 1st Revision. ORNL-5567/R1. Oak Ridge, TN: ORNL.

Yeh, G.T., and V.S. Tripathi. 1989. A critical evaluation of recent developments in hydrogeochemical transport models of reactive multichemical components. Water Resources Research 25(1): 93-108.

Yeh, G.T., and V.S. Tripathi. 1990. HYDROGEOCHEM: A Coupled Model of Hydrological and Geochemical Equilibrium of Multi-Component Systems. ORNL-6371. Oak Ridge, TN: ORNL.

Yeh, G.T., and V.S. Tripathi. 1991. A model for simulating transport of reactive multispecies components: Model development and demonstration. Water Resources Research 27(12): 3075-3094.

Yeh, G.T., and D.S. Ward. 1981. FEMWASTE: A Finite Element Model of WASTE Transport Through Saturated-Unsaturated Porous Media. ORNL-5522. Oak. Ridge, TN: ORNL.

Younker, J.L. et al. 1992. Report of Early Site Suitability Evaluation of the Potential Repository Site at Yucca Mountain, Nevada. SAIC-91/8000, Washington, DC: SAIC.

Zienkiewicz, O.C. 1979. The Finite Element Method. New York, NY: McGraw-Hill.

Zienkiewicz, O.C., and K. Morgan 1983. Finite Element and Approximations. New York, NY: Wiley. 
Zimmerman, R.W., and M.K. Blanford. 1986. Expected thermal and hydrothermal environments for waste emplacement holes based on G-Tunnel heater experiments. 27th U.S. Symposium on Rock Mechanics. H. Hartman, ed. Littleton, CO: SME/AIME: 874-882.

Zimmerman, R.M., M.L. Blanford, J.F. Holland, R.L. Schuch, and W. H. Barrett. 1986a. Final Report: G-Tunnel Sinall-Diameter Experiments. SAND84-2621. Albuquerque, NM: SNL.

Zimmerman, R.M., R.L. Schuch, D.S. Mason, M.L. Wilson, M.E. Hall, M.P. Board, R.P. Bellman, M.P. Blanford. 1986b. Final Report: G-Tunnel Heated Block Experiment. SAND84-2620. Albuquerque, NM: SNL. :

Zyvoloski, G., Z Dash, and S. Kelkar. 1991. FEHMN 1.0: Finite Element Heat and Mass Transfer Code. LA-12062-MS. Los Alamos, NM: LANL. 
APPENDIX A

\section{THERMAL PROCESSES}




\section{THERMAL PROCESSES}

Thermal processes are controlled by the temperature field, which is needed to (i) calculate the thermalmechanical environment to predict preclosure underground opening stability and postclosure stability of emplacement boreholes, (ii) model the thermal-hydrologic environment during operation and after closure of the repository to predict performance of the engineered barrier systems, and (iii) predict the THC environment for radionuclide transport to the accessible environment through the geologic setting.

The thermal loading and the resulting temperature field are expected to have a significant influence on hydrologic, mechanical, and chemical processes, which then influence the underground opening stability and the performance objectives of the repository. It is expected that the placement of heat-generating HLW will elevate the temperature field, cause a redistribution of the in situ water, create thermal-mechanical stresses in the rock, affect container corrosion processes, and alter the geochemical transport of radionuclides. Likewise, the hydrologic, mechanical, and chemical processes have the possibility of influencing the temperature distribution.

For the effects of thermal processes to be predicted, mathematical models need to be established and accepted as accurately representing the thermal physics. In this section, the development of a general mathematical model for heat transfer at the proposed HLW repository is presented. The common assumptions and approximations are discussed, because they lead to special cases of the mathematical models. This section is completed by briefly describing how thermal processes may be influenced by hydrologic, mechanical, or chemical processes.

\section{A.1 MATHEMATICAL MODELS}

A traditional approach has been adopted for presenting the mathematical model in a conservation equation for energy followed by constitutive equations.

\section{A.1.1 Conservation Equation}

The conservation of energy equation is the basis for the mathematical model that is used to predict the temperature distribution. The conservation of energy equation can be written in either the differential (Pollock, 1982, 1986; Whitaker, 1977; Forsyth 1990; Forsyth and Simpson, 1991; Todreas and Kazimi, 1990) or integral (Pruess, 1987; Runchal and Sagar, 1992) form using a variety of defined variables from the literature. The choice of either differential or integral equations is considered to be based on convenience, and the equations can be shown to be equivalent. Similarly, the choice of variable names and definitions is frequently based on convenience. In this work, the conservation of energy equation is presented in differential form

$$
\frac{\partial e_{S L G}}{\partial t}=-\nabla \cdot\left(\vec{q}_{E}+\vec{J}_{E}\right)+Q_{E}
$$

where

$$
\begin{array}{ll}
\mathrm{e}_{\mathrm{SLG}} & =\text { stored energy in solid, liquid, and gas phases }\left(\mathrm{J} / \mathrm{m}^{3}\right), \\
\overrightarrow{\mathrm{q}}_{\mathrm{E}} & =\text { advective flux of energy }\left(\mathrm{W} / \mathrm{m}^{2}\right),
\end{array}
$$


$\begin{array}{ll}\vec{\jmath}_{E} & =\text { diffusive flux of energy }\left(\mathrm{W} / \mathrm{m}^{2}\right), \text { and } \\ \mathrm{Q}_{\mathrm{E}} & =\text { volumetric source/sink of energy }\left(\mathrm{W} / \mathrm{m}^{3}\right) .\end{array}$

In Eq. (A-1), the term on the left-hand side is the local rate of change of stored (or accumulated) energy. The first term on the right-hand side is the divergence of the advective and diffusive fluxes. The last term on the right-hand side is an energy source/sink that may be due to radioactive decay heat, exothermic/endothermic chemical reactions, viscous dissipation of mechanical energy, or a combination of these events.

In the next section, the constitutive equations for the stored energy and energy fluxes are introduced. By introducing mathematical models for these terms, assumptions and approximations are introduced. It is convenient to discuss one assumption that is routinely accepted in thermal analyses. In many discussions (and in this work), the "thermodynamic" internal energy is of primary interest because it is directly related to temperature. Therefore, the energy equation [Eq. (A-1)] is assumed to be valid for thermodynamic energy, which results from the subtraction of the mechanical energy equation from the total energy equation (Todreas and Kazimi, 1990). The changes in kinetic and gravitational-potential energy are assumed to be negligible in comparison to changes in internal energy. In addition, the viscous dissipation is considered negligible.

\section{A.1.2 Constitutive Equations}

In this section, the constitutive equations for the energy equation are presented. The first constitutive equation is for the stored energy, which is expressed as the summation of solid, liquid, and gas phase contributions.

$$
e_{S L G}=(1-\phi) \rho_{S} h_{S}+\phi S_{L} \rho_{L} h_{L}+\phi S_{G} \rho_{G} h_{G}
$$

where

$$
\begin{aligned}
& \mathrm{e}_{\mathrm{SLG}}=\text { stored energy }\left(\mathrm{J} / \mathrm{m}^{3}\right), \\
& \rho_{\mathrm{G}}=\text { gas density }\left(\mathrm{kg} / \mathrm{m}^{3}\right), \\
& \rho_{\mathrm{L}} \quad=\text { liquid density }\left(\mathrm{kg} / \mathrm{m}^{3}\right), \\
& \mathrm{h}_{\mathrm{L}} \quad=\text { liquid enthalpy }(\mathrm{J} / \mathrm{kg}), \\
& \mathrm{h}_{\mathrm{G}} \quad=\text { gas enthalpy }(\mathrm{J} / \mathrm{kg}) \\
& \mathrm{h}_{\mathrm{S}} \quad=\text { solid enthalpy }(\mathrm{J} / \mathrm{kg}) \\
& \phi \quad=\text { porosity }\left(\mathrm{m}^{3} \text { void } / \mathrm{m}^{3}\right. \text { medium) (dimensionless), } \\
& \mathrm{S}_{\mathrm{L}} \quad=\text { liquid saturation }\left(\mathrm{m}^{3} \mathrm{liquid} / \mathrm{m}^{3}\right. \text { void) (dimensionless), and } \\
& \mathrm{S}_{\mathrm{G}} \quad=\text { gas saturation }\left(\mathrm{m}^{3} \text { gas } / \mathrm{m}^{3}\right. \text { void) (dimensionless). }
\end{aligned}
$$

In Eq. (A-2), the enthalpy is used instead of the internal energy which is consistent with other works (Pollock, 1982, 1986). The solid and liquid phase enthalpies and internal energies are approximately equal, because the solid and liquid are approximately incompressible. As such, they are used interchangeably in the literature. For nearly constant gas pressures, the energy associated with volumetric changes is negligible in comparison with the energy associated with temperature changes. The gas phase enthalpy is then approximately equal to the gas phase internal energy, and the distinction between enthalpy and internal energy is frequently neglected. 
The liquid and gas saturations are equal to the fractions of the pore volume occupied by the liquid and gas, respectively. By definition, the saturations sum to unity

$$
S_{L}+S_{G}=1
$$

The advective flux of energy, $\vec{q}_{E}$, is distinguished from the diffusive flux where advection is attributed to the bulk movement of fluid (either liquid or gas phase) and diffusion is attributed to molecular mixing. The advective energy flux consists of liquid and the gas phases contributions

$$
\overrightarrow{\mathrm{q}}_{E}=h_{L} \rho_{L} \vec{v}_{L}+h_{G} \rho_{G} \vec{v}_{G}
$$

where

$$
\overrightarrow{\mathrm{v}}=\text { area-averaged velocity }(\mathrm{m} / \mathrm{s}) \text {. }
$$

The area-averaged fluid velocities are considered parts of the hydrologic process and are discussed in Appendix B; hence, a mathematical model for these fluxes is not presented here.

The diffusive energy flux, $\overrightarrow{\mathrm{J}}_{\mathrm{E}}$, consists of a conductive component and a gas phase mass diffusion component that is neglected here because it has a minor contribution relative to conductive heat transfer (Pollock, 1986). The conductive heat flux is given by Fourier's Law

$$
\overrightarrow{\mathrm{j}}_{\mathrm{E}}=-\mathrm{k}_{\mathrm{T}} \nabla \mathrm{T}
$$

where

$$
\begin{aligned}
& \mathrm{k}_{\mathrm{T}}=\text { thermal conductivity of the medium }[\mathrm{W} /(\mathrm{m}-\mathrm{K})] \text {, and } \\
& \mathrm{T}=\text { temperature }(\mathrm{K}) .
\end{aligned}
$$

The energy source/sink term, $Q_{E}$, in Eq. (A-1) is intended to be very general and may include radioactive decay heat and exothermic/endothermic chemical reactions. The radioactive decay heat associated with high-level radioactive waste can be distinguished between actinide decay reactions and fission product decay reactions (see Figure A-1).

At short times after discharge from a nuclear reactor $(t<100$ yrs), the heat generation is due primarily to fission product decay. At longer times, the heat generation is due primarily to actinide decay reactions. In Figure A-1, the logarithm of relative thermal power is noted to decrease approximately linearly with the logarithm of time, especially for the larger times. In earlier studies of the geologic disposal of HLW, a nominal case for freshly emplaced fuel was assumed to be $10 \mathrm{yrs}$ discharged from the reactor (O'Neal ett al., 1984). In more recent studies (Buscheck and Nitao, 1992), fuels have been assumed with longer decay times (30 and $60 \mathrm{yrs).} \mathrm{With} \mathrm{"cooler"} \mathrm{fuels,} \mathrm{a} \mathrm{larger} \mathrm{number} \mathrm{of} \mathrm{spent} \mathrm{fuel}$ assemblies may be placed in a single disposal container, or the disposal containers may be placed closer together to change the repository areal power density (APD). The potential benefits of high APDs is currently being studied by Buscheck and Nitao (1992), among others. 


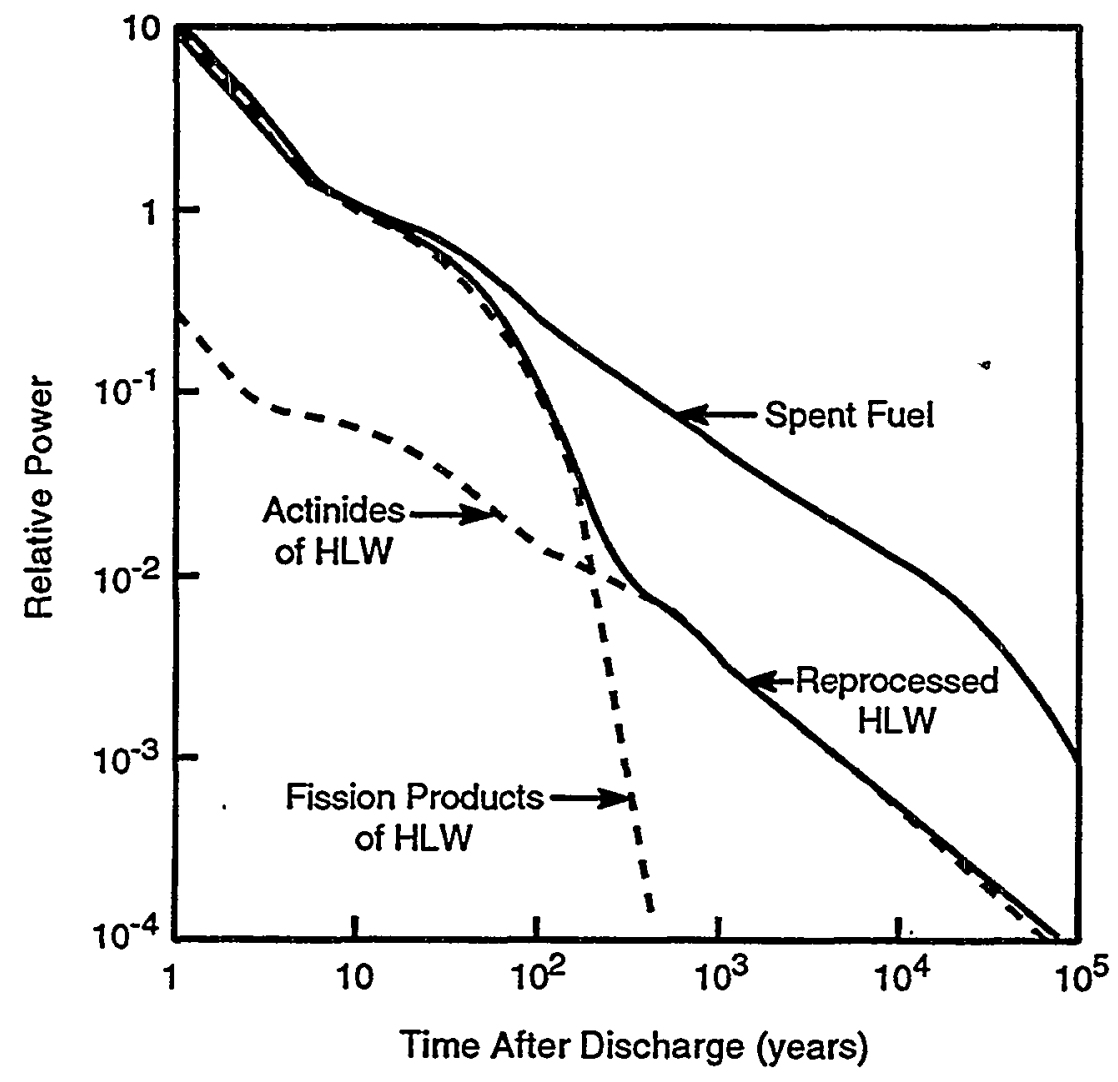

Figure A-1. Relative thermal power of HLW showing the importance of fission products at short times and actinides at long times (Verma and Pruess, 1988; among others).

This summarizes a mathematical model that describes the conservation of energy and related constitutive equations. The mathematical models for the material properties $\left(\rho_{L}, \rho_{G}, h_{L}\right.$, and $\left.h_{G}\right)$ are not presented here, because they are considered straightforward (Pollock, 1986; Green et al., 1992; among others). At this point, it is assumed that the material properties can be calculated. Two simplifications and special cases are presiented.

\section{A.2 SPECIAL CASES FOR THE MATHEMATICAL MODEL}

In the literature, it is common to find studies that are based on subsets of the mathematical model presented in Section A.1. Descriptions, such as "conduction dominated" and "heat pipe," have been used in the literature and represent subsets of the complete model.

\section{A.2.1 Conduction Dominated}

The description, "conduction dominated," is used to indicate that the advective heat transfer is negligible in comparison with the conductive heat transfer

$$
\left|\vec{\jmath}_{E}\right| \gg\left|\vec{q}_{E}\right|
$$


Similarly, changes in the stored energy are assumed to be dominated by the energy stored in the solid phase

$$
\left|\Delta\left[(1-\phi) \rho_{S} h_{S}\right]\right|>\left|\Delta\left(\phi S_{L} \rho_{L} h_{L}\right)\right|+\left|\Delta\left(\phi S_{G} \rho_{G} h_{G}\right)\right|
$$

It is commonly assumed that the internal energy of the solid is linearly related to the temperature, $h_{S}=c_{S}$ $\mathrm{T}$, through the heat capacity, $\mathrm{c}_{\mathrm{S}}$. The thermal conductivity is assumed to be constant, so that the energy equation can be simplified to the heat conduction equation (where, for additional simplicity, the energy source/sink term is neglected, $\mathrm{Q}_{\mathrm{E}}=0$ )

$$
\frac{\partial \mathrm{T}}{\partial \mathrm{t}}=\alpha \nabla^{2} \mathrm{~T}
$$

where

$$
\alpha=\text { thermal diffusivity }\left[\alpha=k_{\mathrm{T}} /\left(\rho_{\mathrm{S}} c_{\mathrm{S}}\right)\right]
$$

The heat conduction equation is considered to be a simpler mathematical model of the energy equation and is frequently used to predict the temperature distribution at the proposed HLW repository (RoglansRibas and Spinrad, 1989; among others). The application of the heat conduction equation is justified when heat conduction is the dominant heat transfer mechanism and the energy storage in the solid phase is the dominant energy accumulation mechanism. For many far-field (tens to hundreds of meters from waste packages) temperature predictions, these assumptions are expected to be valid, especially when liquid flow is negligible (Buscheck and Nitao, 1992).

O'Neal et al. (1984), have calculated the temperature field in waste packages and in the surrounding host rock (see Figure A-2). It was assumed that conduction was the dominant heat transfer mechanism in the host rock, while conduction, natural convection, and thermal radiation were modeled in the spent fuel assemblies, fuel to container gaps, and container to host rock gaps. Although the work of O'Neal et al. is dated, it remains useful in ascertaining the expected temperature profiles near waste packages. Currently, the DOE is exploring repository design options; hence, more exact information is necessary for more exact analyses. The work of O'Neal et al., highlights that a significant volume of the surrounding host rock may reach high temperatures $\left(>100^{\circ} \mathrm{C}\right)$, which will enhance rock dry out.

\section{A.2.2 Heat Pipe}

The term, "heat pipe," has been used in the literature to describe a condition where the dominant heat transfer mechanism is due to vapor and liquid counterflow (Doughty and Pruess, 1992, among others). The liquid water is vaporized at one location and moves by advection to a second location where it condenses. Liquid water is drawn from the region where condensation occurs to where vaporization occurs; hence, there is liquid flow and vapor flow in opposite directions (counterflow). The heat of vaporization required to change phase from liquid to gas creates a net heat flow from the location of vaporization to condensation. It is important to note that the heat pipe effect does not require a temperature gradient, and, therefore, can be isothermal. Also, the traditional heat pipe contains a single species of fluid; hence, in this discussion only, water vapor is considered, and air is assumed not to be present.

In the mathematical model for the conservation of energy [Eq. (A-1)], the heat pipe is typically described as being a steady-state phenomenon 


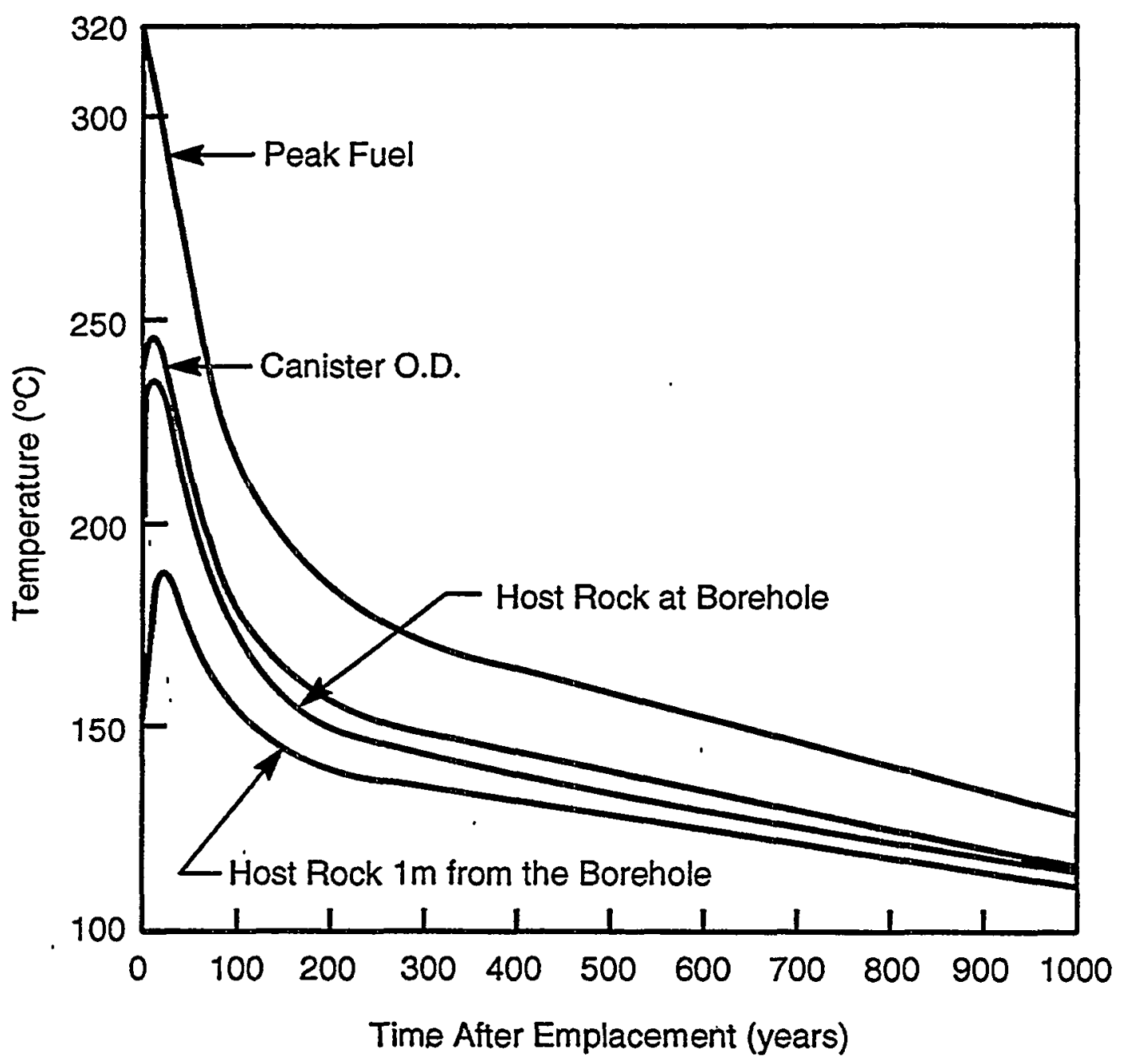

Figure A-2. Typical temperature histories of waste package components and host rock near emplaced spent fuel canister (O'Neal et al., 1984).

$$
\frac{\partial \mathrm{e}_{\text {SLG }}}{\partial \mathrm{t}}=0
$$

in a region which has no heat sources

$$
\mathrm{Q}_{\mathrm{E}}=0
$$

and occurs when advective heat transfer is dominant

$$
\left|\vec{q}_{E}\right| \gg\left|\vec{\jmath}_{E}\right|
$$

The advective fluxes of mass in the liquid and gas phases are equal in magnitude and opposite in direction 


$$
\vec{q}_{W, G}=-\vec{q}_{W, L}
$$

However, due to differences in enthalpy, the advective energy flux is nonzero and can be quite large, based on the magnitude of the mass fluxes

$$
\overrightarrow{\mathrm{q}}_{E}=\mathrm{h}_{\mathrm{G}} \overrightarrow{\mathrm{q}}_{\mathrm{G}}+\mathrm{h}_{\mathrm{L}} \overrightarrow{\mathrm{q}}_{\mathrm{L}}=\mathrm{h}_{\mathrm{LG}} \overrightarrow{\mathrm{q}}_{\mathrm{G}}
$$

where

$$
h_{L G}=\text { enthalpy of vaporization for water }\left(h_{L G}=h_{G}-h_{L}\right)(J / k g) \text {. }
$$

The enthalpy of vaporization for water is quite large $\left(h_{L G} \approx 4200 \mathrm{~J} / \mathrm{kg}\right)$ so that only a small counterflow of fluid is required to transport a large amount of heat with a negligible temperature gradient. The importance of a heat pipe does depend on the ability of water and vapor to move freely in a porous medium; this is discussed in Section 2.2.1 of this report.

\section{A.3 THERMAL PROPERTIES DATABASES}

The most important thermal properties for heat transfer calculations are (i) thermal conductivity, (ii) specific heat, and (iii) density. There has been some characterization of thermal properties at Yucca Mountain (DOE, 1988, 1990). The thermal conductivities are relatively uniform between the different rock units at Yucca Mountain and vary from approximately 1.0 to $2.0 \mathrm{~W} /(\mathrm{m}-\mathrm{K})$. The principal factor influencing thermal conductivity has been reported to be whether the rock is dry or saturated (DOE, 1988). The conductivity is lower for dry conditions compared to saturated conditions. In the literature, either the linear or square-root functions of thermal conductivity as a function of saturation have been used (Pruess, 1987, among others)

$$
\mathrm{k}_{\mathrm{T}}=\mathrm{k}_{\mathrm{T}}\left(\mathrm{S}_{\mathrm{L}}=0\right)+\mathrm{S}_{\mathrm{L}}\left[\mathrm{k}_{\mathrm{T}}\left(\mathrm{S}_{\mathrm{L}}=1\right)-\mathrm{k}_{\mathrm{T}}\left(\mathrm{S}_{\mathrm{L}}=0\right)\right]
$$

or

$$
\mathrm{k}_{\mathrm{T}}=\mathrm{k}_{\mathrm{T}}\left(\mathrm{S}_{\mathrm{L}}=0\right)+\sqrt{\mathrm{S}_{\mathrm{L}}}\left[\mathrm{k}_{\mathrm{T}}\left(\mathrm{S}_{\mathrm{L}}=\cdot 1\right)-\mathrm{k}_{\mathrm{T}}\left(\mathrm{S}_{\mathrm{L}}=0\right)\right]
$$

- Similarly, variation of the heat capacity and density of tuffaceous rock are reported by DOE (1988). Buscheck and Nitao (1992), among others, note that much of the heat transfer at the proposed repository is expected to be conduction dominated. Hence, calculations are expected to be sensitive to both thermal properties and loading conditions.

Wollenberg et al. (1983), note that the thermal conductivity of dry rock can be much lower than saturated rock due to air having a much lower thermal conductivity than water, $\left(\mathrm{k}_{\mathrm{T}, \mathrm{WATER}} \approx 25 \mathrm{k}_{\mathrm{T}, \mathrm{ARR}}\right)$. For simple conductive analyses, tuff has been assumed to have the following characteristics: $\mathrm{k}_{\mathrm{T}} \approx 1.4$ $1.8 \mathrm{~W} /(\mathrm{m}-\mathrm{K}) ; \rho \mathrm{c} \approx 2.0 \times 10^{6} \mathrm{~J} /\left(\mathrm{m}^{3}-\mathrm{K}\right)(\mathrm{DOE}, 1990)$.

In the waste packages, the heat transfer may be due to a combination of conduction, natural convection, and thermal radiation. In order to include conduction heat transfer in an analysis, the thermal 
conductivity must, be known: In order to include natural convection or thermal radiation, more information is typically required. Thermal radiative heat transfer from solid surface to solid surface requires knowledge of the surface emissivities. In a spent fuel assembly, thermal radiation can be the dominant heat transfer mechanism (Manteufel, 1991). In this case, the emissivity of interest would be that of heavily oxidized zircaloy cladding. The authors of MATPRO (Hohorst et al., 1990) have compiled material properties of interest in nuclear applications, which include oxidized zircaloy emissivity (see Figure A-3). The emissivity is frequently assumed to be 0.8 , as shown in Figure A-3. The assumed value of emissivity becomes increasingly important as radiative heat transfer becomes dominant. Siegel and Howell (1992) list emissivities of various engineering materials that may be needed to analyze parts of the engineered barrier.

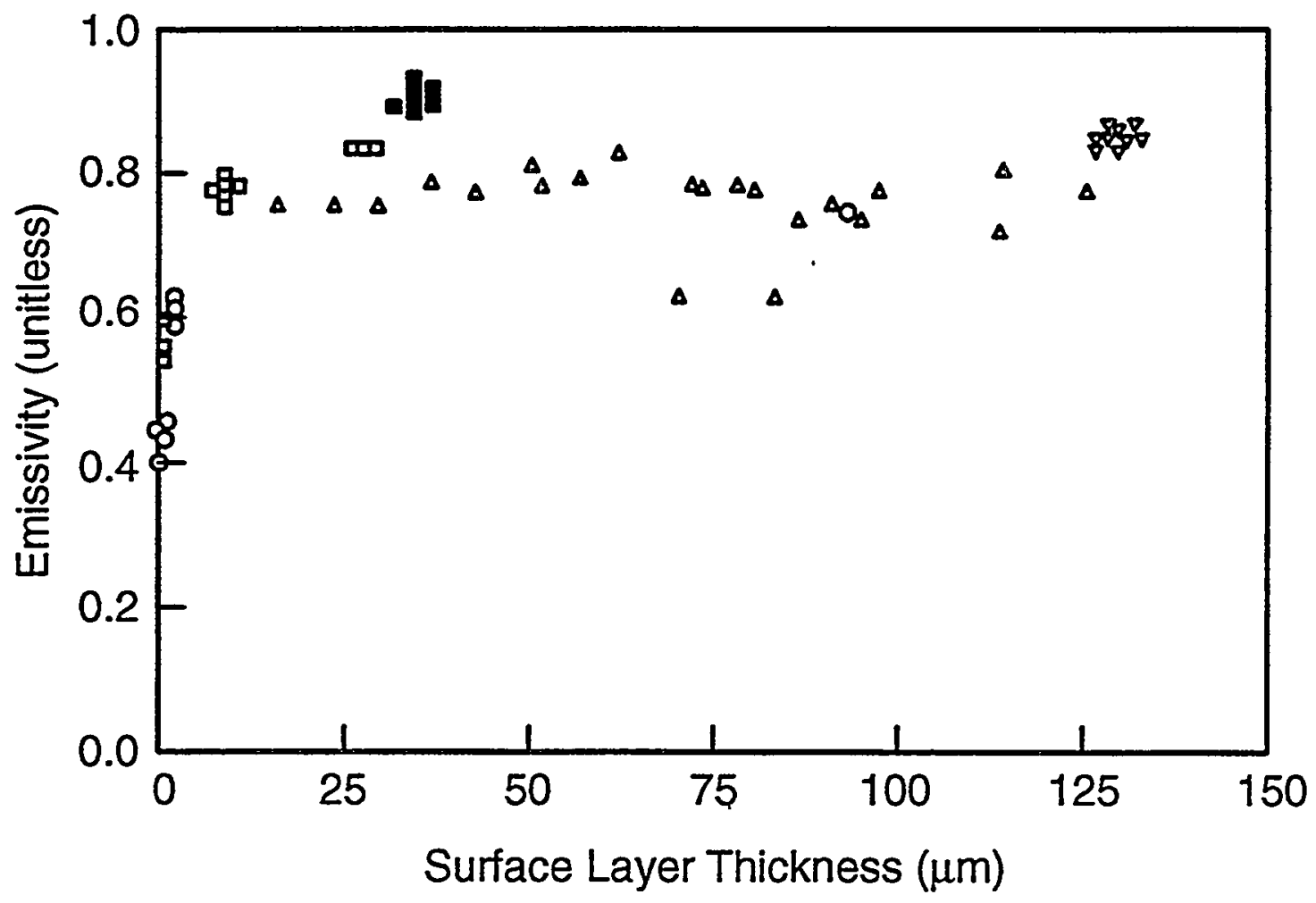

Figure A-3. Comparison of experimental measurements of the emissivity of oxidized zircaloy (spent fuel rods) (Hohorst et al., 1990).

Natural convection is probably the most difficult heat transfer mechanism for which to compile a database of properties. This is because natural convection is primarily governed by the volume/geometry and imposed temperature boundary conditions. Natural convection may be important in large air gaps and analysts should consult texts for pertinent correlations (Incropera and DeWitt, 1981; Lienhard, 1987; Eckert and Drake, 1987; among others). 


\section{A.4 COUPLINGS WITH OTHER PROCESSES}

In this section, the coupling with hydrologic, mechanical, and chemical processes is discussed as this is expected to influence heat transfer processes and the temperature distribution. Each process is discussed in greater detail in Section 2 of this report.

Hydrologic processes can influence the heat transfer through (i) vaporization/condensation of water, (ii) liquid phase advection, and (iii) gas phase advection. The relative importance of fluid movement on heat transfer, however, may not be significant in many cases because conduction heat transfer in the porous medium may be dominant. For comparison, geothermal systems have large fluid flows so that the heat transfer may be convection dominated. For the partially-saturated zone at Yucca Mountain, flow rates are expected to be sufficiently low so that it may be conduction dominated.

Mechanical processes can directly influence the heat transfer through (i) frictional generation of thermal energy or (ii) joint separation, which increases the resistance to heat conduction (possibly expressed as changes in the effective thermal conductivity of the rock mass). A frictional heat source may be present at joints that experience slipping. None of these couplings, however, is expected to have a large impact on thermal processes. Heat transfer is expected to influence mechanical processes through thermally induced stresses (Hodgkinson and Bourke, 1980), which possibly leads to thermally induced microfractures (i.e., cracks) (Wang et al., 1989).

Chemical processes can directly influence heat transfer through the thermal energy source/sink term due to exothermic and endothermic chemical reactions. If the reaction is exothermic, it can be modeled as a thermal energy source. If the chemical reaction is endothermic, it can be modeled as a thermal energy sink. This effect will probably be minor compared to convective heat transfer. The presence of dissolved species may have an effect on fluid vapor pressures, fluid densities, and fluid and rock heat capacities. 


\section{APPENDIX B}

\section{HYDROLOGIC PROCESSES}




\section{HYDROLOGIC PROCESSES}

Hydrologic processes in a fractured, porous medium are presented in this section. The subject of fluid flow in a porous medium is discussed by Bear (1972), Bear and Bachmat (1991), de Marsily (1986), Freeze and Cherry (1979), Hillel (1980a,b), and Domenico and Schwartz (1990), among others. Thermally driven hydrologic processes are of particular interest in this report and are discussed by Forsyth (1990), Forsyth and Simpson (1991), Whitaker (1977), Bixler (1985), Pollock (1982, 1986), Pruess (1987), and Pruess et al., (1990a,b). General references in the area of fluid mechanics include books by Bird et al. (1960), Fox and McDonald (1978), Potter and Foss (1982), and Todreas and Kazimi (1990).

The hydrologic processes important in underground disposal of HLW are those processes associated with fluid transport in both the gaseous and liquid phases, for both water and air in a fractured porous medium. In the literature, this is frequently referred to as two-phase (gas and liquid), two-component (air and water) mass transfer (Forsyth, 1990). Throughout this work, air is referred to as a component for modeling convenience, however, it is noted that air is a mixture of $\mathrm{N}_{2}, \mathrm{O}_{2}, \mathrm{CO}_{2}$, and other gaseous species.

The mathematical model presented here focuses on fluid transport in a homogeneous and isotropic porous medium. Additional mathematical models are required to represent fractures that are expected to be important at the proposed HLW repository.

\section{B.1 A MATHEMATICAL MODEL}

The mathematical model for hydrologic processes in a homogeneous and isotropic porous medium is based on the principles of (i) the balance of momentum and (ii) the conservation of mass (Fox and McDonald, 1978; Bear, 1972; Pruess, 1987; Forsyth, 1990; Whitaker, 1977; Pollock, 1982, 1986). In a porous medium, the balance of momentum is expressed as Darcy's law and is frequently considered a constitutive equation. The conservation of mass equations are more general and provide the foundation for the mathematical model of hydrologic processes. The conservation equations will be discussed, followed by a discussion of the constitutive equations.

\section{B.1.1 Conservation Equations}

For this discussion the amount of air in the liquid phase is assumed negligible. The conservation equation for air can be written as:

$$
\frac{\partial\left(\phi S_{G} \rho_{A, G}\right)}{\partial t}=-\nabla \cdot \rho_{A, G} \vec{v}_{G}-\nabla \cdot \vec{j}_{A, G}+Q_{A, G}
$$

for liquid water

$$
\frac{\partial\left(\phi S_{L} \rho_{W, L}\right)}{\partial t}=-\nabla \cdot \rho_{W, L} \vec{v}_{L}-Q_{W, L G}+Q_{W, L}
$$

and for water vapor

where 


$$
\frac{\partial\left(\phi S_{G} \rho_{W, G}\right)}{\partial t}=-\nabla \cdot \rho_{W, G} \vec{v}_{G}-\nabla \cdot \vec{J}_{W, G}+Q_{W, L G}+Q_{W, G}
$$

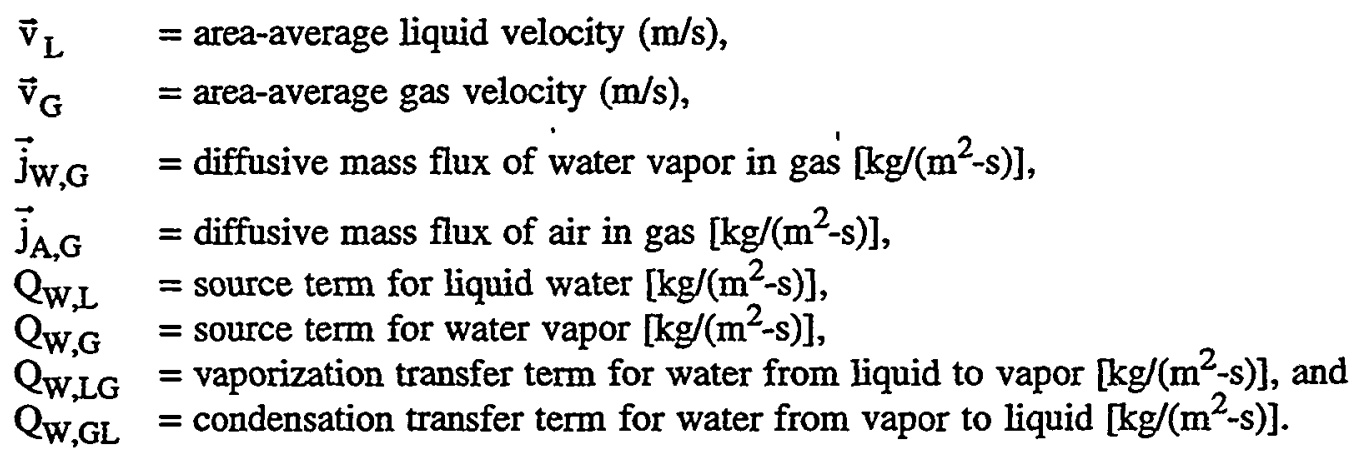

Equations (B-2) and (B-3) require a constitutive equation for the liquid-to-gas phase mass transfer, $\mathrm{Q}_{W, L G}$. The phase densities and velocities can be functions of temperature, chemical composition, and mechanical stresses, leading to various types of couplings with other processes. If the equations are added, then a single mass conservation equation is obtained:

$$
\begin{gathered}
\frac{\partial\left(\phi S_{L} \rho_{W, L}+\phi S_{G} \rho_{W, G}\right)}{\partial t}=-\nabla \cdot\left(\rho_{W, L} \vec{v}_{L}+\rho_{W, G} \vec{v}_{G}\right) \\
-\nabla \cdot \vec{\jmath}_{W, G}+Q_{W, L}+Q_{W, G}
\end{gathered}
$$

Equation (B-4) is considered useful when the density of water in the gas phase, $\rho_{W, G}$, can be determined through a constitutive equation. Typically, the condition of local thermal-hydrologic equilibrium is assumed so that the amount of water in the gas phase is determined by the temperature and saturation (Edlefsen and Anderson, 1943). Equations (B-2) and (B-3) represent the case where the water in both the liquid and gas phase are not required to be in equilibrium, and Eq. (B-4) represents the equilibrium case.

\section{B.1.2 Constitutive Equations}

The constitutive equations need to be presented for the advective mass fluxes, the diffusive mass fluxes, and the interphase mass transfer terms (for the nonequilibrium model). In this discussion, the components in each of the phases are assumed to be locally well-mixed so that each component travels at the same velocity. This appears to be a standard assumption that is made throughout the literature (Bear, 1972). The phase velocities are based on a modified Darcy's law, which is assumed valid for partiallysaturated conditions (Bear, 1972; Hillel, 1980a) 


$$
\begin{gathered}
\vec{v}_{L}=-\frac{k_{S A T} k_{R E L, L}}{\mu_{L}}\left(\nabla P_{L}+\rho_{L} g k\right) \\
\vec{v}_{G}=-\frac{k_{S A T} k_{R E L, G}}{\mu_{G}}\left(\nabla P_{G}+\rho_{G} g \mathbb{K}\right)
\end{gathered}
$$

where

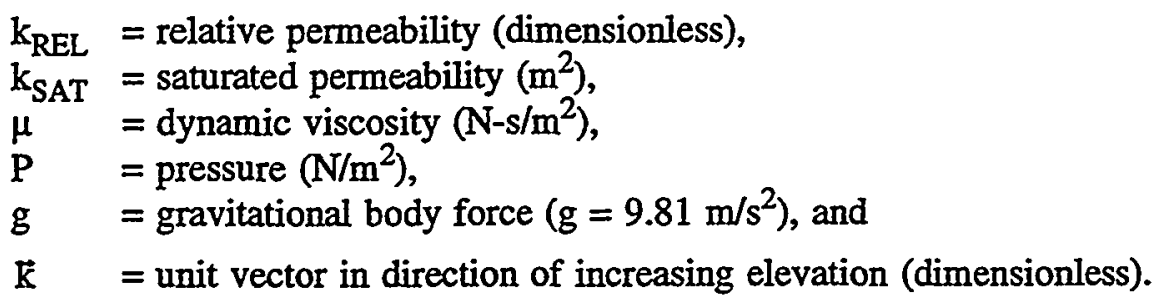

For partially-saturated conditions, $P_{L}$ should be considered as a "potential energy of liquid water per unit volume." The potential energy of water in a partially-saturated medium is based on capillary and adsorptive forces. The gas pressure and liquid pressure are related through the capillary/adsorptive pressure:

$$
\mathrm{P}_{\text {CAPIADS }}=\mathrm{P}_{\mathrm{G}^{-}}-\mathrm{P}_{\mathrm{L}}
$$

where

$$
\mathrm{P}_{\text {CAP/ADS }}=\text { capillary/adsorptive pressure }\left(\mathrm{N} / \mathrm{m}^{2}\right) .
$$

The $P_{\text {CAP/ADS }}$ is frequently characterized as a function of $S_{L}$ using the van Genuchten model (van Genuchten, 1978; 1980). The molecular diffusion mass fluxes are given by Fick's law

$$
\begin{gathered}
\vec{J}_{\mathrm{W}, \mathrm{G}}=-\tau \phi S_{\mathrm{G}} \mathrm{D}_{\mathrm{WA}, \mathrm{G}} \rho_{\mathrm{G}} \nabla \mathrm{m}_{\mathrm{W}, \mathrm{G}} \\
\overrightarrow{\mathrm{J}}_{\mathrm{A}, \mathrm{G}}=-\tau \phi \mathrm{S}_{\mathrm{G}} \mathrm{D}_{\mathrm{WA}, \mathrm{G}} \rho_{\mathrm{G}} \nabla \mathrm{m}_{\mathrm{A}, \mathrm{G}}
\end{gathered}
$$

where

$$
\begin{aligned}
& \tau \quad=\text { tortuosity (dimensionless), } \\
& D_{\mathrm{WA}, \mathrm{G}}=\text { molecular diffusion coefficient of water vapor in the gas }\left(\mathrm{m}^{2} / \mathrm{s}\right), \\
& \mathrm{m}_{\mathrm{W}, \mathrm{G}}=\text { mass fraction of water vapor in the gas (dimensionless), and } \\
& \mathrm{m}_{\mathrm{A}, \mathrm{G}}=\text { mass fraction of air in the gas (dimensionless). }
\end{aligned}
$$

\section{B.1.3 Material Properties}

The air and water densities $\left(\rho_{\mathrm{A}, \mathrm{G}}, \rho_{\mathrm{W}, \mathrm{G}}, \rho_{\mathrm{W}, \mathrm{L}}\right)$ are considered to be material properties that depend on temperature, pressure, and, possibly, chemical composition. 
The total gas pressure, $P_{G}$, is equal to the sum of component partial pressures

$$
P_{G}=P_{A, G}+P_{W, G}
$$

The air and water components of the gas phase are assumed to behave as ideal gases

$$
\begin{aligned}
\rho_{A, G} & =\frac{P_{A, G} M_{A}}{R_{I} T} \\
\rho_{W, G} & =\frac{P_{W, G} M_{W}}{R_{I} T}
\end{aligned}
$$

where

$$
\begin{array}{ll}
\mathrm{P}_{\mathrm{W}, \mathrm{G}} & =\text { partial pressure of water vapor in the gas }\left(\mathrm{N} / \mathrm{m}^{2}\right), \\
\mathrm{P}_{\mathrm{A}, \mathrm{G}} & =\text { partial pressure of air in the gas }\left(\mathrm{N} / \mathrm{m}^{2}\right), \\
\mathrm{M}_{\mathrm{A}} & =\text { molecular weight of air }(28.97 \mathrm{~g} / \mathrm{mole}), \\
\mathrm{M}_{\mathrm{W}} & =\text { molecular weight of water }(18.02 \mathrm{~g} / \mathrm{mole}) \\
\mathrm{R}_{\mathrm{I}} & =\text { ideal gas constant }[8.314 \mathrm{~J} /(\text { mole- } \mathrm{K})], \text { and } \\
\mathrm{T} & =\text { temperature }(\mathrm{K}) .
\end{array}
$$

As a result of Eqs. (B-10), (B-11), and (B-12), the total gas density is equal to the sum of component densities

$$
\rho_{\mathrm{G}}=\rho_{\mathrm{A}, \mathrm{G}}+\rho_{\mathrm{W}, \mathrm{G}}
$$

\section{B.2 DATABASES FOR HYDROLOGIC PROPERTIES OF YUCCA MOUNTAIN}

The hydrologic properties pertinent to the proposed HLW repository are discussed in a number of sources (Montazer and Wilson, 1984; Peters et al., 1984; DOE, 1988; Klavetter and Peters, 1986; Flint and Flint, 1990, and others). The primary quantities of interest are (i) location and dimensions of stratigraphic units, (ii) matrix permeability and porosity, (iii) fracture density, apertures, orientations, and connectivity, and (iv) moisture retention curves for matrix and fracture (i.e., $P_{C A P / A D S}$ versus $S_{L}$ curves).

An east-west, cross-sectional view of the proposed repository is illustrated in Figure B-1. The primary hydrogeologic units are: (i) Tiva Canyon welded (TCw), (ii) Paintbrush nonwelded (PTn), (iii) Topopah Spring welded (TSw), (iv) Calico Hills nonwelded vitric (CHnv), and (v) Calico Hills nonwelded zeolitic (CHnz) (DOE, 1988). The thicknesses vary and are approximately 0-150, 20-100, 290-360, 100400 , and 100-400 meters, respectively (Montazer and Wilson, 1984).

The proposed repository is to be located in the welded Topopah Spring unit at Yucca Mountain. The rock in this unit is reported to have a low porosity, and low permeability, and is densely fractured 


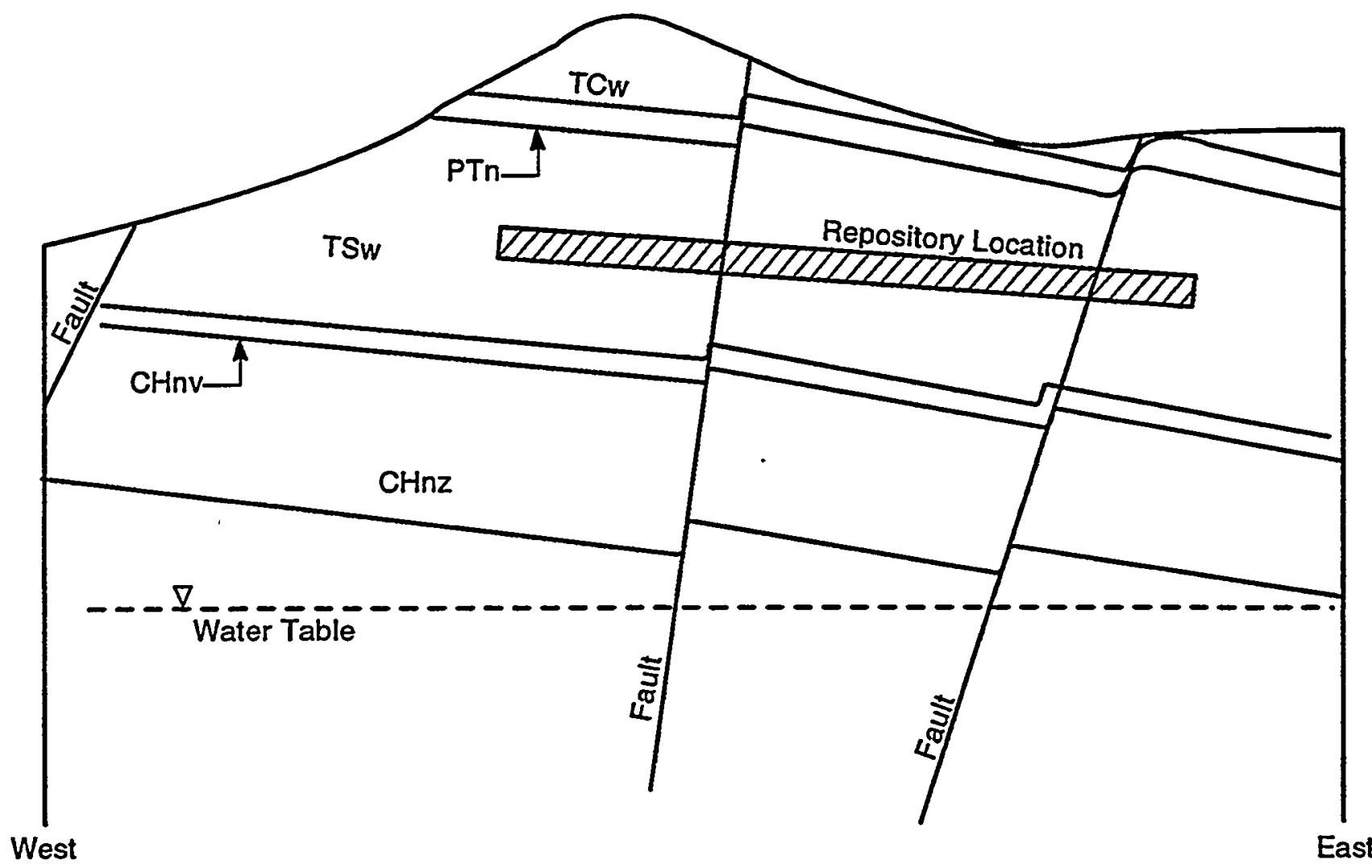

TCw Tiva Canyon Welded

PTn Paintbrush Nonwelded

TSw Topopah Spring Welded

CHnv Calico Hills Nonwelded Vitric

CHnz Calico Hills Nonwelded Zeolitic

Figure B-1. East-west, cross-sectional illustration highlighting the different hydrogeologic units in the unsaturated zone at Yucca Mountain (adapted from DOE, 1988).

(Scott et al, 1982; Klavetter and Peters, 1986). The other units have varying hydrologic properties and are compared in Table B-1. The earliest and most extensive testing of tuffaceous materials was performed by Peters et al. (1984). The hydrogeologic units at Yucca Mountain generally fall into two categories: (i) welded tuffs and (ii) nonwelded tuffs. Typically the welded tuffs have low matrix permeability $\left(-10^{-18} \mathrm{~m}^{2}\right)$, low-to-medium matrix porosity, $(-10 \mathrm{percent})$ and high fracture densities $\left(>10\right.$ fractures $\left./ \mathrm{m}^{3}\right)$. Conversely, nonwelded tuffs have higher matrix permeability $\left(-10^{-14} \mathrm{~m}^{2}\right)$, high matrix porosity (3040 percent) and low fracture densities $\left(<5\right.$ fractures $\left./ \mathrm{m}^{3}\right)$. Most of the fractures are "steeply dipping" and nearly vertical (DOE, 1990).

The stratification of rock units and the difference in permeability, porosity, and fracture characteristics increase the complexity of predicting hydrologic processes. Wang (1992) reports that the variations in hydrologic parameters from tuff samples span 2 to 4 orders of magnitude for saturated permeability $\left(\mathrm{k}_{\mathrm{SAT}}\right)$, which highlights the uncertainties in hydrologic properties. 
Table B-1. Summary of hydrogeologic properties relevant to Yucca Mountain (adapted from DOE, 1988).

\begin{tabular}{|c|c|c|c|c|c|}
\hline $\begin{array}{l}\text { Hydro- } \\
\text { geologic } \\
\text { Unit }^{\mathbf{a}}\end{array}$ & $\begin{array}{c}\text { Source } \\
\text { of } \\
\text { Data }\end{array}$ & $\begin{array}{l}\text { Fracture } \\
\text { Aperture } \\
\text { (microns) }\end{array}$ & $\begin{array}{c}\text { Fracture } \\
\text { Density } \\
\left(\text { no } J \mathbf{m}^{3}\right)^{c}\end{array}$ & $\begin{array}{l}\text { Matrix } \\
\text { Porosity }\end{array}$ & $\begin{array}{c}\text { Saturated } \\
\text { Matrix } \\
\text { Permeability } \\
\left(\mathbf{m}^{\mathbf{2}}\right)\end{array}$ \\
\hline \multirow[b]{2}{*}{$\mathrm{TCw}$} & (d) & $\mathrm{ND}^{\mathrm{b}}$ & $10-20$ & 0.12 & $2 \times 10^{-18}$ \\
\hline & (e) & 6.74 & ND & 0.08 & $1 \times 10^{-18}$ \\
\hline \multirow[b]{2}{*}{ PTn } & (d) & ND & 1 & 0.46 & $1.0 \times 10^{-14}$ \\
\hline & (e) & 27.00 & ND & 0.40 & $4.0 \times 10^{-14}$ \\
\hline \multirow[b]{2}{*}{ TSw } & (d) & ND & $8-40$ & 0.14 & $3.6 \times 10^{-18}$ \\
\hline & (e) & 5.13 & $\mathrm{ND}$ & 0.11 & $1.9 \times 10^{-18}$ \\
\hline \multirow[b]{2}{*}{ CHnv } & (d) & ND & $2-3$ & 0.37 & $5.1 \times 10^{-15}$ \\
\hline & (e) ${ }^{\prime}$ & 15.50 & ND & 0.46 & $2.8 \times 10^{-14}$ \\
\hline \multirow[b]{2}{*}{$\mathrm{CHnz}$} & (d) & ND & $2-3$ & 0.31 & $9.2 \times 10^{-18}$ \\
\hline & (e) & 15.50 & ND & 0.28 & $2.0 \times 10^{-18}$ \\
\hline \multicolumn{6}{|c|}{$\begin{array}{l}\text { a Hydrogeologic units are shown in Figure B-1 } \\
\text { b ND = no data } \\
\text { c Scott et al. (1982) } \\
\text { d Montazer and Wilson (1984) } \\
\text { e Peters et al. (1984) } \\
\text { Note: } 1 \mathrm{~m}^{2} \text { (permeability) } \propto 10^{7} \mathrm{~m} / \mathrm{s} \text { (hydraulic conductivity) }\end{array}$} \\
\hline
\end{tabular}

\section{B.3 FRACTURES}

One major difficulty in predicting hydrologic processes is the characterization of fractures and fracture networks (Sagar and Gureghian, 1991; Ababou, 1991; Rasmussen and Evans, 1987, 1989; Klavetter and Peters, 1985; Wang and Narasimhan, 1986). By itself, the subject of flow in fractures has received much attention because fractures have different geometries and hydrologic properties from the matrix. Fractures are typically described as being $2 \mathrm{D}$ with an aperture width typically of $0.1-1.0 \mathrm{~mm}$. Sagar and Gureghian (1991) describe five candidate modeling approaches for predicting flow in a fractured, porous medium: (i) equivalent continuous porous medium model, (ii) double porosity model, (iii) discrete fracture network model, (iv) discrete fracture equilibrium model, and (v) discrete fracture nonequilibrium model. Only the first approach is discussed here. The reader is referred to Sagar and Gureghian (1991) for a more complete discussion of the different modeling approaches. 
The equivalent continuous porous medium model has been used by Pruess, et al. (1987; 1990a,b), Rockhold et al. (1992), Nitao et al. (1992), Peters and Klavetter (1988), Nitao (1988), Buscheck and Nitao (1992), and others. The effective continuum approach uses an effective porosity

$$
\phi_{\mathrm{EFF}}=\phi_{\mathrm{F}}+\left(1-\phi_{\mathrm{F}}\right) \phi_{\mathrm{M}}
$$

where

$$
\begin{aligned}
& \phi_{\mathrm{EFF}}=\text { effective continuum porosity (dimensionless) } \\
& \phi_{\mathrm{F}}=\text { fracture porosity (based on a parallel plate model) (dimensionless), and } \\
& \phi_{\mathrm{M}}=\text { matrix porosity (dimensionless) }
\end{aligned}
$$

and an effective saturation

$$
S_{\mathrm{EFF}}=\frac{S_{\mathrm{F}} \phi_{\mathrm{F}}+S_{\mathrm{M}}\left(1-\phi_{\mathrm{F}}\right) \phi_{\mathrm{M}}}{\phi_{\mathrm{EFF}}}
$$

where

$$
\begin{aligned}
& \mathrm{S}_{\mathrm{EFF}}=\text { effective continuum saturation (dimensionless), } \\
& \mathrm{S}_{\mathrm{F}}=\text { fracture saturation (dimensionless), and } \\
& \mathrm{S}_{\mathrm{M}}=\text { matrix saturation (dimensionless) }
\end{aligned}
$$

Klavetter and Peters (1986), describe a weighting procedure to calculate the effective matrix-fracture permeability

$$
\mathrm{k}_{\mathrm{EFF}}=\mathrm{k}_{\mathrm{M}}\left(1-\phi_{\mathrm{F}}\right)+\mathrm{k}_{\mathrm{F}} \phi_{\mathrm{F}}
$$

where

$$
\begin{aligned}
& k_{E F F}=\text { effective continuum permeability }\left(\mathrm{m}^{2}\right) \\
& k_{\mathrm{F}}=\text { fracture permeability }\left(\mathrm{m}^{2}\right), \text { and } \\
& \mathrm{k}_{\mathrm{M}}=\text { matrix permeability }\left(\mathrm{m}^{2}\right) .
\end{aligned}
$$

The effective continuum approach yields characteristic curves for hydraulic conductivity (or permeability) as a function of pressure head (or capillary/adsorptive pressure). Figure B-2 shows the effective continuum permeability as a function of saturation, showing influences of matrix and fracture flow, where the upper plateau represents the effect of the fractures and the lower plateau the effect of the matrix. At a pressure head near zero, both the fracture network and matrix are saturated. At higher pressure head, the fractures desaturate (dry out), and the conductivity is reduced by approximately three orders of magnitude (the hump). As the pressure head increases, the matrix dries, and the conductivity is continually reduced. Typically, the effective continuum model predicts that the fractures quickly dry out at an effective continuum saturation below 98 percent for the repository horizon at Yucca Mountain (e.g., Nitao, 1988). This implies that the fractured medium can sustain a considerable increase in liquid content before fracture flow occurs. The consensus in the literature is that the effective continuum approach is accurate as long as the hydrologic processes are sufficiently slow so that the fracture and matrix are in hydrologic equilibrium (Ababou, 1991; Gureghian and Sagar, 1991; Klavetter and Peters, 1986; Pruess et al., 1990a,b; and Nitao et al., 1992, among others). 


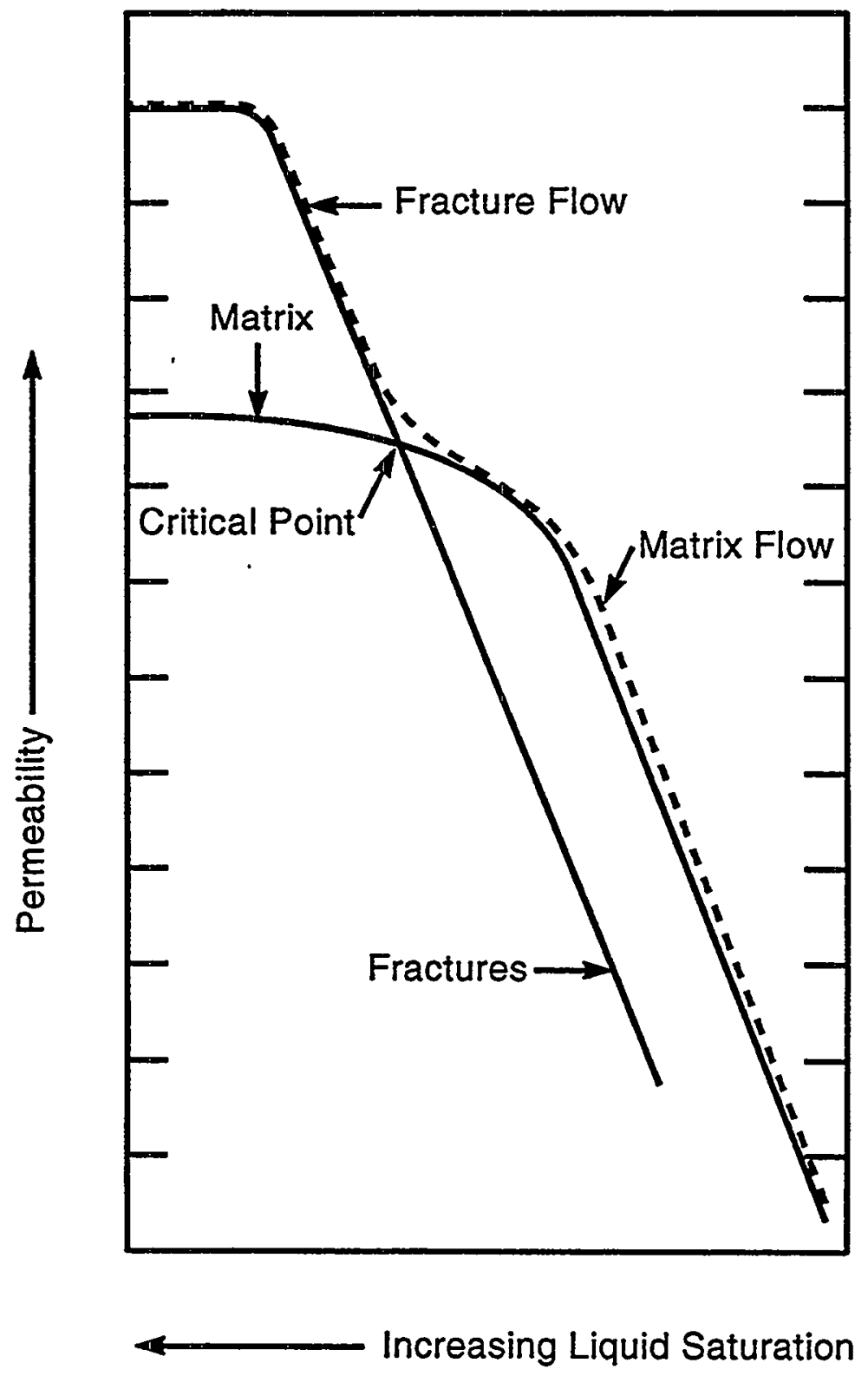

Figure B-2. Effective continuum permeability as a function of saturation showing influences of matrix and fracture flow (Montazer and Wilson, 1984; Domenico and Schwarz, 1990; among others). 
Buscheck and Nitao (1992) compare calculated and measured liquid saturation profiles for various steady-state, 1D recharge fluxes (see Figure B-3). The saturation data are from the Yucca Mountain Project Reference Information Base (DOE, 1990) and are shown in Figure B-3 as data points with error bars. The calculated liquid saturation profiles are based on an equivalent (or effective) continuum model (ECM). Buscheck and Nitao (1992) note that the ECM predictions and measurements are in good agreement in the low matrix permeability units (predominantly welded units). The ECM underpredicts the saturation in the high matrix permeability units (predominantly nonwelded) units. Nonequilibrium fracture flow through the welded units is a likely explanation of the differences (Buscheck and Nitao, 1992). Another explanation may be spatial variability of in situ saturation so that the measured values are biased due to localized sampling in boreholes. Another explanation is that the ground surface recharge flux is highly nonuniform so that episodic flows prevent Yucca Mountain from reaching a meaningful "steady-state" saturation profile. One possible explanation is that the ECM is not appropriate in low permeability units where episodic, fracture-dominated flow may dominate the hydrology. Wang and Narasimahan (1985), Peters and Klavetter (1988), Nitao (1991), and Nitao et al. (1992), among others have used and have noted limitations of the ECM. The consensus is that the ECM is valid under low fluxes and slow transients and cannot predict transient fracture flow that occurs before equilibration. Significant fracture flow occurs in the ECM model only when the matrix is nearly saturated; therefore, there is severe underprediction of the amount of fracture flow during episodic conditions, which are characterized by rapid regionally partially-saturated transients and large infiltration rates.

The importance of fracture flow in a rock has been experimentally observed. Nitao, et al. (1992), reviewed experimental data that suggest that fracture-dominated flow has occurred at Yucca Mountain. The data include the detection of chlorine-36 as well as tritium at depths $450-500$ feet below the ground surface due to episodic rainfalls (Nitao et al., 1992). Polymer-based drilling fluid flowed between adjacent boreholes (USW G-1 and USW UZ-1) indicating fluid movement in fractures occurred over a significant distance in less than 3 yrs.

In general, large fractures lead to larger fracture flow rates; however, more liquid is required to saturate the fracture. In many cases, a fracture may not be fully saturated; however, liquid may flow in the fracture. In this case, the terminology of liquid "dripping" in fractures may be more appropriate.

Another consideration in predicting liquid flow in fractures is the connectivity of fracture networks. In general, a well connected network will lead to greater penetration (or travel) of liquid. Here, the emphasis is on vertical fractures, where horizontal fractures may provide connectivity between vertical fractures.

\section{B.4 COUPLINGS WITH OTHER PROCESSES}

Hydrologic processes are widely expected to be important in the transport of radionuclides; hence, they are expected to be considered in the study of chemical processes. The importance of chemical processes on hydrologic processes has been described mainly as dissolution and precipitation that may alter hydrologic properties such as fracture aperture.

The couplings with thermal processes are also discussed in the literature; however, thermal

processes are expected to influence hydrologic processes stronger than hydrologic processes affecting 


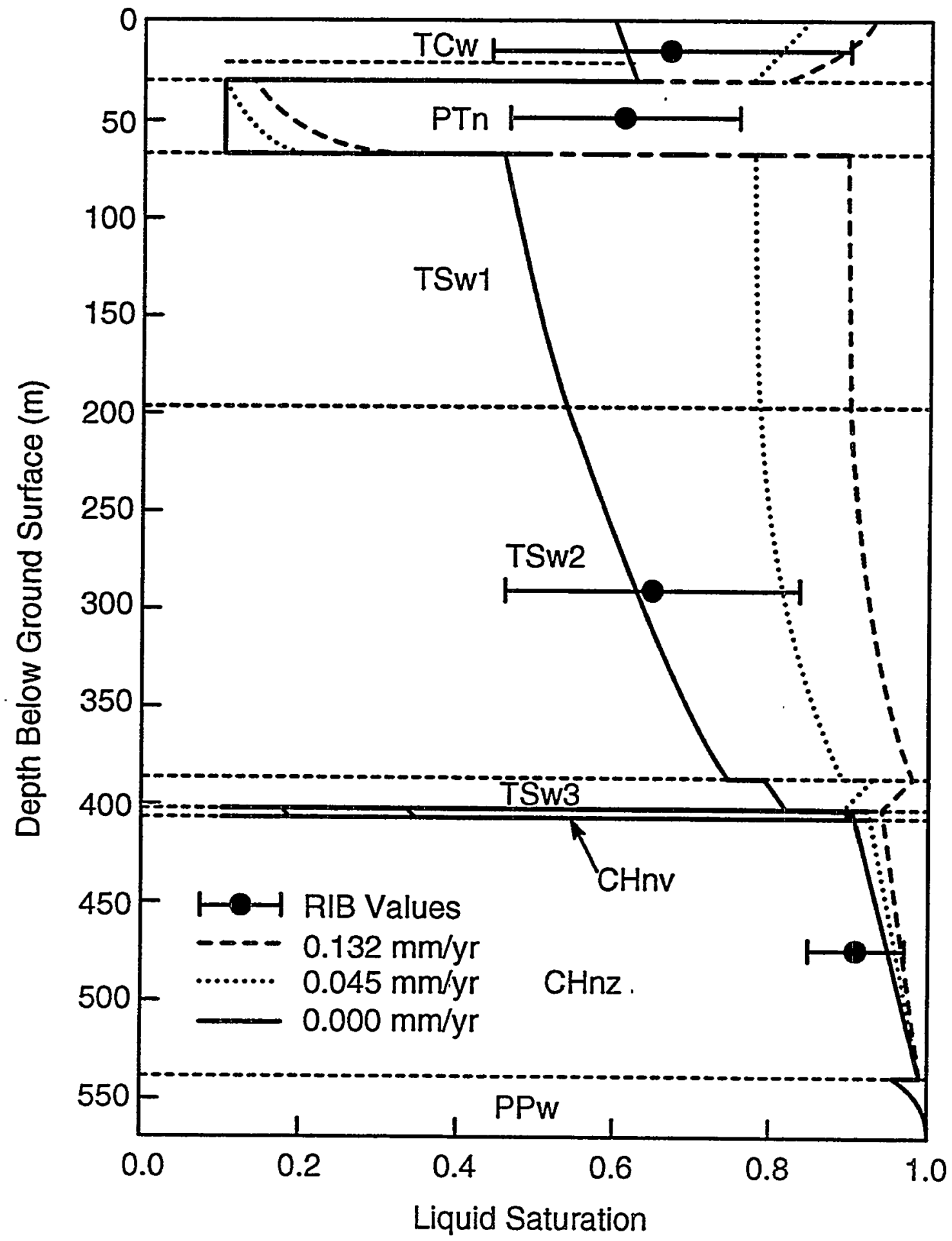

Figure B-3. Comparison of calculated and measured liquid saturation profiles for various steadystate, 1D recharge fluxes (Buscheck \& Nitao, 1992). Calculated values based on an effective continuum model and measured values are from the Reference Information Base (RIB) (DOE, 1990). 
thermal processes. A possible exception is the "heat pipe" (Doughty and Pruess, 1988; 1990; 1991; 1992; Pruess et al., 1990a,b).

The mechanical properties of jointed rock may be described as a function of saturation; however, this effect continues to be investigated. The Early Site Suitability Evaluation (Younker et al., 1992) suggests that compressive strength is $20-30$ percent less for saturated versus dry samples. The changes in the mechanical stresses and strains in the rock are expected to change fracture apertures and influence flow (e.g., seismic pumping). 
APPENDIX C

MECHANICAL PROCESSES 


\section{MECHANICAL PROCESSES}

Maintaining preclosure stability of the underground openings and postclosure stability of waste emplacement boreholes, in addition to allowing the option for waste retrieval, are key issues in the design of the repository. A typical rock mass can be viewed as a complex system of joints, faults, and intact rock. In some cases, the jointing may exist in several well defined orientations. However, in the vicinity of underground openings, additional excavation-induced fracturing can occur depending on the in situ stress conditions and type of excavation method, creating a complicated fracture and joint network. Also, anticipated and unanticipated natural phenomena, such as earthquake activity and underground nuclear testing, are particularly important at the proposed repository at Yucca Mountain and need to be taken into account in the design. Mechanical deformation within the rock mass consists of intact rock deformation, closure and shearing of joints, and movements along faults. Material properties of the intact rock at the proposed Yucca Mountain repository will most likely be heterogeneous due to the nature of rock formation. Also, different sets of joints are likely to have different strengths and may also deform differently under various loading conditions.

Laboratory experimentation of the mechanical properties of the welded tuff at Yucca Mountain (Price et al., 1987) indicates that the intact rock is quite strong, with a uniaxial compressive strength of approximately $160 \mathrm{MPa}$ and a high deformation modulus. Uncracked samples have stress-strain curves that show nearly elastic behavior up until failure. Samples with cracks exhibit nonlinear stress-strain behavior as expected when the applied stress is above 50 percent of the failure stress (Price, et al., 1987).

\section{C.1 CONTINUUM MATERIAL BEHAVIOR}

Numerous textbooks describe the mechanics of continuous media, for example, Sokolnikoff (1956), Charlez (1991), Love (1944), Nowacki (1962), and Timoshenko and Goodier (1970). It can be shown from momentum balance that the general equations of motion can be expressed as

$$
\nabla \cdot \underline{\underline{\sigma}}+\overrightarrow{\mathrm{f}}=\rho \frac{\mathrm{d} \overrightarrow{\mathrm{v}}}{\mathrm{dt}}
$$

where

$$
\begin{aligned}
\underline{\underline{\sigma}} & =\text { stress tensor }\left(\mathrm{N} / \mathrm{m}^{2}\right), \\
\overrightarrow{\mathrm{f}} & =\text { body force vector }\left(\mathrm{N} / \mathrm{m}^{3}\right) \\
\rho & =\text { solid density }\left(\mathrm{kg} / \mathrm{m}^{3}\right), \\
\overrightarrow{\mathrm{v}} & =\text { velocity vector }(\mathrm{m} / \mathrm{s}), \text { and } \\
\mathrm{t} & =\text { time }(\mathrm{s}) .
\end{aligned}
$$

When the inertial effects are negligible, Eq. (C-1) reduces to

$$
\nabla \cdot \underline{\underline{\sigma}}+\overrightarrow{\mathrm{f}}=0 \text {. }
$$

or alternatively using index notation as

where the repeated index ( $j$ ) denotes a summation, and the comma preceding the index $(j)$ denotes partial differentiation with respect to that index. Equation $(C-3)$ represents three equations for $i=1,2$, and 3 , 


$$
\sigma_{\mathrm{ji}, \mathrm{j}}+\mathrm{f}_{\mathrm{i}}=0
$$

known as the "equilibrium equations," which must be satisfied at any point within the medium. These equilibrium equations are used as the basis for solving elastostatic problems in rock mechanics.

The state of strain at a point in the body is specified by the components of the strain tensor. These components give the normal and shear strains for infinitesimal line elements originally parallel to the coordinate axes. The six equations for the strain components can be written in index notation as

$$
e_{i j}=\frac{1}{2}\left(u_{i, j}+u_{j, i}\right)
$$

where

$e_{i j}=$ normal strain components for $i=j$ or shear strain components for $i \neq j$, and $u_{i}=$ three components of displacement $\left(u_{1}, u_{2}, u_{3}\right)$.

Equation (C-4) shows that the six strain components can be written in terms of the three displacements, $u_{1}, u_{2}$, and $u_{3}$. It follows that the strain components cannot be specified independently of one another and that some relationship must exist between them. It is possible using Eq. (C-4) to establish a set of six relations between the strains known as "compatibility equations," (e.g., Charlez, 1991). These compatibility equations ensure that the strain field and the corresponding displacement field are continuous across the medium. Equations $(\mathrm{C}-3)$ and $(\mathrm{C}-4)$ or the corresponding compatibility equations are general and must be satisfied for any type of material. The six components of stress, six components of strain, and three components of displacement are thus written in terms of the three equilibrium [Eq. (C-3)] and six strain displacement equations [Eq. (C-4)], resulting in a set of nine equations for fifteen unknowns. The remaining six equations are provided by the constitutive relations between the stresses and strains and depend on the type of material behavior.

Intact rock behavior, especially that which exhibits high strength properties, such as the welded tuff at the proposed Yucca Mountain repository, can be approximated quite accurately by linear elastic behavior with the possible exception of those welded tuff units that have a very high porosity. This type of behavior is usually written in terms of the so-called generalized Hooke's Law, which states that at each point in a 3D, linearly elastic body, the six components of the stress tensor are linearly related to the six components of the strain tensor. These stress-strain relations for an isotropic, linearly elastic material can be written in index notation as

$$
e_{i j}=\frac{1}{2 G}\left[\sigma_{i j}-\frac{v_{P}}{1+v_{P}} \sigma_{k k} \delta_{i j}\right]
$$

where

$$
\begin{aligned}
& \mathrm{G}=\text { shear modulus }\left(\mathrm{N} / \mathrm{m}^{2}\right) \\
& v_{\mathrm{P}}=\text { Poisson's ratio (dimensionless), } \\
& \delta_{\mathrm{ij}}=\text { Kronecker delta }\left(\delta_{\mathrm{ij}}=1 \text { if } \mathrm{i}=\mathrm{j}, \text { and } \delta_{\mathrm{ij}}=0 \text { for } \mathrm{i} \neq \mathrm{j}\right)(\text { dimensionless }) \text {, and } \\
& \sigma_{\mathrm{kk}}=\text { sum of normal stresses }\left(\sigma_{\mathrm{kk}}=\operatorname{Trace}(\sigma)=\sigma_{11}+\sigma_{22}+\sigma_{33}\right)\left(\mathrm{N} / \mathrm{m}^{2}\right) .
\end{aligned}
$$


In Eq. (C-5), $\mathrm{k}$ is a dummy index, and, because it is repeated, denotes the sum of the three stress components. From moment equilibrium conditions, it is found that the stress tensor is symmetric, $\sigma_{\mathrm{ij}}=\sigma_{\mathrm{ji}}$, and, consequently, from Eq. (C-5) the strain tensor is also symmetric.

\section{C.2 ROCK JOINT BEHAVIOR}

In many situations, deformation along the rock joints in the form of normal and shear displacements plays a larger role in the deformation of the overall rock mass than just that of the intact rock. An example would be rock mass deformation in a competent, highly jointed rock mass under moderate to low in situ stresses (i.e., shallow depth). The proposed setting for the geologic repository at Yucca Mountain is in a well jointed, competent welded tuff at moderate depth, and thus, deformation along the joints and faults as a result of excavation as well as natural phenomena would be an important part of the overall response of the rock mass. Thus, accurate predictions of the mechanical behavior require knowledge of the rock joint behavior.

Several joint models are currently being used to describe the normal and shear deformation along rock joints. These consist mainly of the Coulomb friction, Barton-Bandis (Barton and Bandis, 1982; Barton et al., 1985), and continuous-yielding joint models (Cundall and Lemos, 1988), as well as ubiquitous or compliant joint models, which are based on a continuum description of the rock mass (Morland, 1974; Gerrard, 1982; Chen, 1986, 1990). The simplest joint model is the Coulomb friction, linear deformation model. For normal loading, elastic reversible closure is allowed up to some limiting normal displacement, and joint separation is allowed when the normal stress is less than the joint tensile strength. For shear loading, the shear displacement is linear and reversible up to a limiting shear stress (determined by the normal stress) followed by perfectly plastic behavior. Shear load reversal after plastic yield is accompanied by permanent shear displacement and hysteresis. The relation between limiting shear resistance and normal stress is given as

$$
\tau=c+\sigma_{n} \tan \left(\phi_{\mathrm{JF}}\right)
$$

where

$$
\begin{aligned}
& \tau \quad=\text { joint shear strength }\left(\mathrm{N} / \mathrm{m}^{2}\right), \\
& \sigma_{\mathrm{n}}=\text { joint normal stress }\left(\mathrm{N} / \mathrm{m}^{2}\right), \\
& \phi_{\mathrm{IF}}=\text { joint friction angle (radians), and } \\
& c \quad=\text { cohesion }\left(\mathrm{N} / \mathrm{m}^{2}\right) .
\end{aligned}
$$

The Coulomb friction, linear deformation joint model is most appropriate for smooth discontinuities that are nondilatant in shear, such as faults at residual strength.

Mechanical experimentation of single rock joints shows the deformation to be nonlinear and inelastic (Bandis, et al., 1983). Barton et al. (1985), have proposed a joint model that describes the normal and shear responses based on testing of single-jointed rock specimens. This model is referred to as the Barton-Bandis joint model. The model takes into account the hysteresis that occurs during joint normal loading-unloading, as well as permanent inelastic closure that takes place after repeated loading and unloading of the joint. The model also allows for dilation of the joint to occur during shearing, as observed in laboratory experiments. The joint shear strength envelope proposed by Barton and Bandis can be expressed using Eq. (C-6) with a variable $\phi_{\mathrm{JF}}$. This $\phi_{\mathrm{JF}}$ is intended to take into account joint damages 
and reduction in the dilation angle during joint shear. In the Barton-Bandis model, the $\phi_{\mathrm{JF}}$ is assumed to be constant, provided the normal stress is constant, during the process of shearing until a peak joint shear displacement corresponding to the peak shear resistance is exceeded. The peak angle of friction of a joint can be expressed in terms of joint roughness and wall strength parameters, as follows

$$
\phi_{\text {peak }}=J \mathrm{RC}_{\text {peak }} \cdot \log _{10}\left(\mathrm{JCS} / \sigma_{\mathrm{n}}\right)+\phi_{\mathrm{r}}
$$

where

$$
\begin{array}{ll}
\phi_{\text {peak }} & =\text { peak (or maximum) joint friction angle (radians), } \\
\mathrm{RC}_{\text {peak }} & =\text { empirically determined initial joint roughness coefficient (dimensionless), } \\
\mathrm{JCS} & =\text { empirically determined joint wall compressive strength }\left(\mathrm{N} / \mathrm{m}^{2}\right), \text { and } \\
\phi_{\mathrm{r}} & =\text { residual joint friction angle (radians). }
\end{array}
$$

Substituting Eq. (C-7) back into Eq. (C-6) and assuming no cohesion, gives the following relation for the peak shear strength

$$
\tau_{\text {peak }}=\sigma_{\mathrm{n}} \tan \left[\mathrm{JRC} \mathrm{Ceak}_{\text {pea }} \cdot \log _{10}\left(\mathrm{JCS} / \sigma_{\mathrm{n}}\right)+\phi_{\mathrm{r}}\right]
$$

The gradual reduction of the shear strength in the post-peak phase is caused by a decline in the effective contribution of roughness due to surface mismatch and wear. This behavior can be modeled by using different values for the roughness, $\mathrm{JRC}_{\mathrm{mob}}$, that will be activated once the joint becomes mobilized. Barton et al. (1985), also proposed an empirical expression defining the joint closure under normal stress as

$$
\sigma_{\mathrm{n}}=\frac{\Delta \mathrm{V}_{\mathrm{j}}}{\mathrm{a}-\mathrm{b} \Delta \mathrm{V}_{\mathrm{j}}}
$$

where $\Delta \mathrm{V}_{\mathrm{j}}$ is the joint closure, and a and $\mathrm{b}$ are empirical constants.

The continuous-yielding joint model was formulated by Cundall and Lemos (1988) to take into account nonlinear compression, nonlinearity and dilation in shear, and a nonlinear limiting shear strength criterion. This model suggests that at any state of normal stress, $\sigma_{n}$, the joint shear strength is given by

$$
\tau=\sigma_{\mathrm{n}} \tan \left(\phi_{\mathrm{JF}}\right) \sin \left(\Delta \mathrm{u}_{\mathrm{s}}\right)
$$

where

$$
\Delta \mathrm{u}_{\mathrm{S}}=\text { joint shear displacement increment. }
$$

As damage accumulates, the $\phi_{\mathrm{JF}}$ is continuously reduced by the following equation

$$
\Delta \phi_{\mathrm{JF}}=\frac{1}{\mathrm{R}}\left(\phi_{\mathrm{JF}}-\phi_{\mathrm{r}}\right) \Delta \mathrm{u}_{\mathrm{s}}^{\mathrm{p}}
$$

where

$R=$ roughness parameter for the joint surface $(m)$, and $\Delta \mathrm{u}_{\mathrm{s}}^{\mathrm{P}}=$ plastic joint shear displacement which is a fraction of $\Delta \mathrm{u}_{\mathrm{S}}$.

Joint stiffnesses for the continuous-yielding model are normal stress dependent according to 


$$
\begin{aligned}
& \mathrm{K}_{\mathrm{n}}=\mathrm{a}_{\mathrm{n}} \sigma_{\mathrm{n}}^{e_{\mathrm{n}}} \\
& \mathrm{K}_{\mathrm{s}}=\mathrm{a}_{\mathrm{s}} \sigma_{\mathrm{n}}^{e_{\mathrm{s}}}
\end{aligned}
$$

where $K_{n}$ is the joint normal stiffness, $K_{s}$ is the joint shear stiffness, and $a_{n}, e_{n}, a_{s}$, and $e_{s}$ are empirical constants.

\section{C.3 MATHEMATICAL MODELING OF MECHANICAL PROCESSES}

There are numerous computational methods currently available for modeling the mechanical deformation of rock. These consist of continuum methods such as finite difference (Forsythe and Warsow, 1960), finite element (Zienkiewicz, 1979; Cook, 1981), and boundary element (Brebbia, 1978) methods. Other approaches consist of the discontinuum (or discrete) modeling technique that includes the distinct element method (Cundall, 1971), modal method (Williams, et al., 1985), discontinuous deformation analysis method (Shi and Goodman, 1988), and moment exchange method (Hahn, 1988). It has been common in engineering practice to model the far-field rock mass behavior using continuum modeling techniques. In this region, one is interested in more of the macroscopic mechanical behavior of the rock mass, or perhaps, deformation along a major structural feature, such as a fault. Discontinuum methods have been used in modeling the near-field mechanical behavior of the rock, where one is more interested in the deformation along individual joints or fractures that may intersect, for example, underground openings or waste emplacement boreholes. Hybrid modeling techniques that combine both continuum and discontinuum techniques have also been used for excavation problems involving infinite and semi-infinite regions. A brief discussion of each of these modeling approaches and computer codes is given below.

\section{C.3.1 Continuum Analysis}

Continuum modeling techniques describe the rock mass as a continuous solid, within which displacements and stresses are defined in terms of governing differential equations. In finite difference and finite element methods, a mesh of elements that are interconnected at the nodes is constructed. Displacement compatibility is required along the edges of the elements. The system of equations is written for the entire assemblage of elements, including the constraints and boundary conditions. Each element of the discretized medium is assigned a constitutive material behavior representative of the medium. Both finite element and finite difference methods are well established for continuum modeling and can be used to model complex material behavior.

In boundary element formulations, only the boundary of the medium (i.e., surface of the excavation or ground surface) is discretized. As a result, the dimension of the problem is reduced by one. The numerical solution is first obtained along these surfaces, and then the solution at different points within the medium is obtained from the solution at the boundary. From a computational point of view, the boundary element leads to a much smaller system of algebraic equations than a finite element solution for the same problem. In addition, the boundary element method is especially well suited to problems involving infinite and semi-infinite regions, such as those occurring in underground excavation design, since the boundary conditions at infinity are met analytically. However, currently available "infinite elements" now allow problems involving semi-infinite and infinite regions to be modeled accurately using the finite element method. The main disadvantage of the boundary element method is that it does not have 
the capability to model complex material behavior as can be done with finite element, finite difference, and finite region methods.

A discontinuous medium, such as a fractured and jointed rock medium, can be analyzed by continuum methods, such as finite element, finite difference, and boundary element methods, by approximating the medium as an equivalent continuum, considering the effective properties in shear and in compression. As the spacing of the joints increases or the joints become stiffer both in compression and in shear, the properties of the rock mass approach the intact rock properties. In such a case, the effect of joint discontinuity is neglected, and the rock mass is modeled as a medium of intact rock. However, with decreasing joint spacing and with decreasing joint stiffness both in shear and in compression, the ratio of rock mass properties to intact rock properties decreases. For these fractured rock masses, the concept of "effective"' or macroscopically averaged properties is used (Fossum, 1985; Amadei and Goodman, 1983; Duncan and Goodman, 1968). This means that one seeks to determine the effective properties that govern the macroscopic behavior of the discontinuous rock masses in terms of the properties of the intact rock and joints and the geometric nature of their combination.

Many finite element (Key, 1987; Whirley, 1991; Hallquist, 1988), boundary element (Banerjee and Ahmad, 1985), and finite difference (Hofmann, 1981b) programs have interface elements or "slide lines" that enable them to model discontinuous materials to some extent. However, their formulation is usually restricted in one or more of the following ways (Cundall and Hart, 1989). First, the logic may break down when many intersecting interfaces are used; second, there may not be an automatic scheme for recognizing new contacts; third, the formulation may be limited to small displacements and rotations. Such programs are usually adapted from existing continuum programs.

\section{C.3.2 Discontinuum Analysis}

Discontinuum modeling techniques have been introduced recently for simulating the complete behavior of a fractured and jointed rock' medium where the discontinuities play a critical role in determining the deformation, including rigid body motion. These techniques model the properties of both joints and the intact rock explicitly. The discrete element models have two distinguishing features compared to continuum models: (i) the behavior of the geologic system is described by both a continuum material description of the intact rock and a discontinuum material model for major discontinuities (i.e., joints, faults, etc.) and (ii) the deformation mechanisms include large displacement (i.e., joint slip and separation) and block rotation. The discrete element method is similar to the finite element or finite difference method in that the problem domain is divided into a system of solid elements (blocks). The principal difference is that the discrete element method also permits the geometry of the elements to be defined by the spacing and orientation of the discontinuities in the rock mass, thereby allowing blocks to interact (or disconnect) from neighboring blocks. The discrete element method includes not only continuum theory representation for the blocks, but also force displacement laws that specify forces between blocks and a motion law that specifies motion of each block due to unbalanced forces acting on the block.

In the discrete element method, blocks may be rigid or deformable. The rigid block formulation represents the medium as a set of discrete blocks that do not change their geometry as a result of applied loading and only the joints can deform. Consequently, the formulation is most applicable to problems in which the deformation of the system is primarily a result of deformation or slip along the discontinuities and where elastic properties of the intact material may be ignored. Such conditions arise in low-stress environments or where the intact material possesses high strength and low deformability. At the site of 
the proposed geologic repository at Yucca Mountain, although the welded tuff is considered to possess high strength, the waste emplacement horizon is at sufficient depth to induce some elastic block deformation around the underground openings. For these situations, the deformation of individual blocks cannot be reasonably ignored; that is, blocks cannot be assumed to be rigid. In this case, arbitrary deformation of blocks is permitted through internal discretization of blocks into finite element or finite difference zones, in addition to the rigid-body modes associated with each block of the jointed rigid block formulation.

\section{C.3.3 Hybrid Analysis}

Hybrid modeling techniques can also be used for analysis of the mechanical behavior in underground excavation problems, which usually involve infinite or semi-infinite regions. Development of this computational scheme is based on a recognition of different deformation modes that occur in rock surrounding an excavation. The far-field away from the excavation experiences only small deformations and is represented reasonably well as an elastic continuum using a boundary element scheme or an infinite element (Bettess and Zienkiewicz, 1977; Zienkiewicz and Morgan, 1983) scheme. The near-field rock in the vicinity of the excavation experiences larger displacements along joints or fractures and possibly nonlinear material behavior, and is better modeled by finite element, finite difference, or discrete element methods. In the hybrid modeling approach, the near-field scheme (finite element, finite difference, or discrete element) is coupled with the far-field scheme (boundary' element or infinite element) by satisfying the condition of continuity of displacements and stresses on the interface between the near-field and the far-field domain. Pan and Reed (1991) show an application of such a coupled distinct element-finite element method towards large deformation analysis of rock masses.

\section{C.4 COUPLING WITH OTHER PROCESSES}

Mechanical processes will, in general, be coupled with thermal, hydrologic, and chemical processes. It is expected that thermal loading as a result of waste emplacement will have a significant effect on the mechanical state within the rock mass, inducing additional stresses and displacements as a result of thermal expansion. The thermal effect will most likely peak before one hundred years, after which it will gradually taper off (e.g., Figure A-2). Changes in chemistry of the rock as a result of thermal heating may cause additional mechanical degradation of the rock around the underground openings and emplacement boreholes.

For the proposed Yucca Mountain site, mechanical processes will likely have a significant effect on the hydrologic characteristics in the near-field repository region, mainly due to changes in the hydraulic apertures around the excavations and emplacement boreholes. However, due to the partially-saturated nature of the Yucca Mountain site, which may introduce gas or vapor phases, and the fact that flow in the fractures may only occur periodically, the mechanical hydrologic coupling is not well understood at this time. 


\section{APPENDIX D}

\section{CHEMICAL PROCESSES}




\section{CHEMICAL PROCESSES}

The performance of both the engineered and natural barriers of a geologic high-level radioactive waste repository can be affected significantly by the operation of chemical processes in response to a variety of external and internal changes. The following section is intended as a brief summary of the principles and applications of equilibrium and kinetic chemical processes pertinent to the proposed HLW repository at Yucca Mountain, Nevada. The reader is referred to a variety of textbooks and review articles (e.g., Garrels and Christ, 1965; Lasaga and Kirkpatrick, 1981; Stumm and Morgan, 1981; Drever, 1982; Lasaga, 1984; Hem, 1985) for a more extensive and complete treatment of equilibrium and kinetic chemistry. The first subsection deals with equilibrium chemical processes. Nonequilibrium reaction kinetics will be discussed in more detail later in this appendix.

The laws of thermodynamics, reaction kinetics, and conservation of energy, mass, and charge are the basic tools for modeling chemical processes (Rubin, 1983; Mangold and Tsang, 1991). A primary distinction is drawn between those processes that are both reversible and "fast enough" to justify an assumption of equilibrium and those that are slow and irreversible, requiring consideration of nonequilibrium reaction kinetics (Figure D-1). Within both equilibrium and nonequilibrium classes of reactions, a secondary distinction is made between homogeneous reactions (single-phase) and heterogeneous (multiple-phase) reactions. A final division can be made for heterogeneous reactions where surface (sorption, ion exchange) and non surface (precipitation/dissolution, redox) processes are discriminated, although there is considerable overlap between these final classes. The ensuing discussion is generally concerned only with the high-order distinction between equilibrium and nonequilibrium chemical processes.

\section{D.1 EQULIBRIUM CHEMICAL PROCESSES}

A geochemical system can generally be represented by a set of simultaneous of reactions (homogeneous or heterogeneous, surface or non surface) of the general form

$$
b B_{(s)}+c C_{(a q)} \Rightarrow d D_{(a q)}+e E_{(g)}
$$

where

$\mathrm{B}, \mathrm{C}, \mathrm{D}$, and $\mathrm{E} \quad=$ phases involved in the reaction [solid(s), aqueous(aq), gas(g)], and $\mathrm{b}, \mathrm{c}, \mathrm{d}, \mathrm{e} \quad=$ stoichiometric reaction coefficients.

By convention, these reactions are written so that the forward reaction proceeds from left to right, and the reverse reaction proceeds from right to left.

For the Jth component in reactions of the type presented in Eq. (D-1), the mass balance is constrained such that

$$
\mathrm{m}_{\mathrm{t}, \mathrm{J}}=\Sigma v_{\mathrm{J}} \mathrm{m}_{\mathrm{J}, \mathrm{I}}
$$

where

$\mathrm{m}_{\mathrm{t}, \mathrm{J}}=$ total molality (moles $/ \mathrm{kg} \mathrm{H}_{2} \mathrm{O}$ ) of component $\mathrm{J}$,

$\mathrm{m}_{\mathrm{J}, \mathrm{I}}=$ molality of species I containing component $\mathrm{J}$ (moles $/ \mathrm{kg} \mathrm{H}_{2} \mathrm{O}$ ), and

$v_{J}=$ stoichiometry of component $J$ in species $I$. 


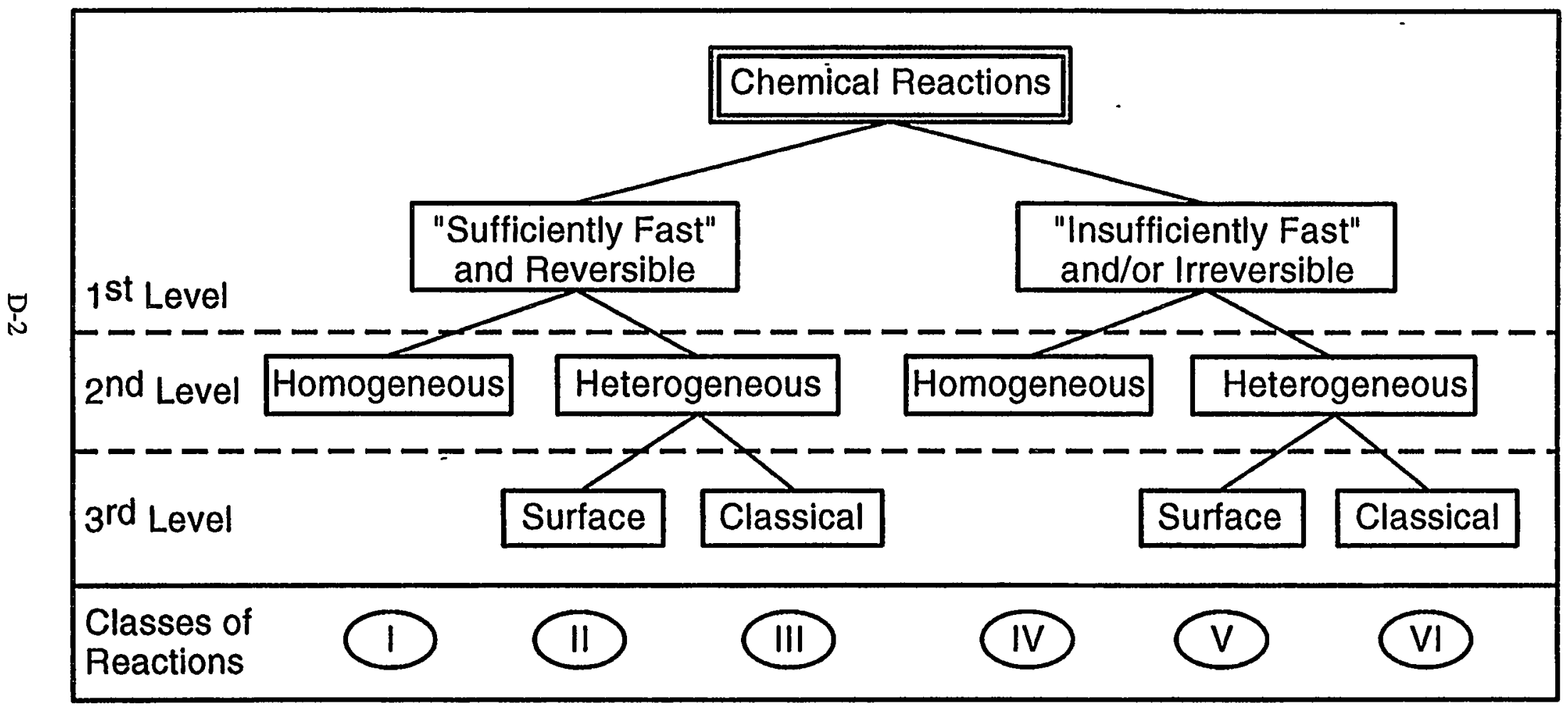

Figure D-1. Distinctions between classes of chemical reactions (adapted from Rubin, 1983). 
In a similar fashion, charge balance in electrolyte solutions is constrained such that

$$
\Sigma z_{\mathbf{I}} m_{I}=0
$$

where

$\mathrm{z} \quad=$ valence charge of the subscripted species, and

$\mathrm{m}_{\mathfrak{I}}=$ molality of species $\mathrm{I}\left(\right.$ moles $/ \mathrm{kg} \mathrm{H} \mathrm{H}_{2} \mathrm{O}$ ).

Based on an assumption of equilibrium, reactions of the form given in Eq. (D-1) can be represented by mass-action equations of the form

$$
K=\frac{\left(a_{D}\right)^{d}\left(f_{E}\right)^{e}}{\left(a_{B}\right)^{b}\left(a_{C}\right)^{c}}
$$

where

$$
\begin{aligned}
\mathrm{K}= & \text { thermodynamic equilibrium constant (dimensionless), which is a function of } \\
& \text { temperature and pressure, } \\
\left(f_{\mathrm{I}}\right)^{\mathrm{i}}= & \text { fugacity }\left(\mathrm{N} / \mathrm{m}^{2}\right) \text { of a gas phase I raised to the power of its reaction coefficient } \mathrm{i} \text {, and } \\
\left(\mathrm{a}_{\mathrm{I}}\right)^{\mathrm{i}}= & \text { the thermodynamic activity (dimensionless) of aqueous species, solid, or liquid I } \\
& \text { raised to the power of its reaction coefficient, } \mathrm{i} \text {. }
\end{aligned}
$$

For pure solids and liquids, $\left(a_{S}\right)=1$, and at low pressures, the fugacity of a gas (in this case, gas $E$ ) can be approximated by its partial pressure, $p$, such that $[p(E)]^{e} \approx\left(f_{E}\right)^{e}$. For aqueous species, however, activity is a function of pressure, temperature, and solution composition, and the activity/molality relationships are generally of the form

$$
\left(a_{I}\right)^{i}=m_{I} \gamma_{I}
$$

where

$$
\gamma_{\mathrm{I}}=\text { the activity coefficient of species } \mathrm{I}\left(\mathrm{kg} \mathrm{H}_{2} \mathrm{O} / \text { mole } \mathrm{I}\right) \text {. }
$$

$\gamma_{\text {I }}$ also varies as a function of temperature, pressure, and solution composition, and is either determined experimentally or defined by one of several approximations, such as the mean-salt method or the DebyeHückel and Davies equations (Stumm and Morgan, 1981). The validity of these approximations is generally limited to relatively dilute solutions. Recent studies (Pitzer, 1973, 1979; Harvie et al., 1984; Plummer et al., 1988; Pabalan and Pitzer, 1990) have developed a set of virial equations (Pitzer equations) to calculate solution properties for concentrations ranging from dilute electrolyte solutions to highly concentrated brines. However, all activity/concentration relations require additional thermodynamic data. In the absence of experimental data, frequently an activity coefficient of 1 is assumed, and simple concentrations are substituted for activities in Eq. (D-4).

The driving force for a chemical reaction, the Gibbs free energy of reaction $\Delta G_{R}(\mathrm{~J} / \mathrm{mole})$, is defined as the difference between the free energy of the products and the reactants. A function of pressure 
and temperature, $\Delta G_{R}$, can also be used (Mangold and Tsang, 1991) to describe chemical equilibrium through the relationship

$$
\Delta G_{R}=\Delta G_{R}^{0}+R_{I} T \ln \left(\frac{\left(a_{D}\right)^{d}[p(E)]^{e}}{\left(a_{B}\right)^{b}\left(a_{C}\right)^{c}}\right)=\Delta G_{R}^{o}+R_{I} T \ln (Q)
$$

where

$$
\begin{array}{ll}
\mathrm{R}_{\mathrm{I}} & =\text { the ideal gas constant }(8.314 \mathrm{~J} / \mathrm{K}-\text { mole }), \\
\mathrm{T} & =\text { the absolute temperature in Kelvin, } \\
\Delta \mathrm{G}_{\mathrm{R}}{ }^{\mathrm{O}} & =\text { the standard free energy change of the reaction (J/mole), and } \\
\mathrm{Q} & =\text { the activity product or the reaction quotient. }
\end{array}
$$

Analogous to $\Delta G_{R}, \Delta G_{R}{ }^{\circ}$ is defined as the difference between the sum of the standard state energies of formation of the products and that of the reactants (e.g., $\Delta G_{R}^{0}=\Sigma \Delta G_{F \text { prods }}^{0}-\Sigma \Delta G_{F}^{0}$ reacts).

Because the reaction will tend towards a minimum energy state, a negative value for $\Delta G_{R}$ will favor the forward reaction; a positive value favors the reverse reaction. As the reaction progresses in the favored direction at a given temperature and pressure, the free energy of reaction $\left(\Delta G_{R}\right)$ will approach zero. At equilibrium, $\Delta G_{R}=0, Q=K$, and $\Delta G_{R}{ }^{\circ}=-R_{I} T[\ln (K)]$. Using thermodynamic relations such as the Maxwell and van't Hoff equations (Garrels and Christ, 1965), and standard state thermodynamic properties [e.g., heat capacity $\left(c_{p}\right)$, enthalpy $(H)$, and entropy $(S)$ ] for the reactant and product phases to calculate the effects of changing conditions on $\Delta \mathrm{G}_{\mathrm{R}}{ }^{\circ}$, it is possible to calculate equilibrium constants as a function of pressure and temperature from Eq. (D-6). Values for $\Delta G_{R}{ }^{\circ}$ are tabulated in a number of thermodynamic databases for a wide variety of liquids, gases, solids, and aqueous species.

Acid-base and redox reactions are written in a form analogous to Eq. (D-1), involving either the transfer of a proton (acid-base) or a hypothetical electron (redox) (Mangold and Tsang, 1991). It is also possible to write redox reactions in terms of the redox species present in solution. This is perhaps a better approach as it assures conservation of electrons for the overall system (Lichtner, 1988). A mathematical construction similar to Eqs. (D-4) and (D-6) can then be used to express these types of reactions as functions of hydrogen ion activity $(\mathrm{pH})$ and oxidation potential (Eh) or electron potential $\left[\mathrm{pE}=(\mathrm{F} / 2.303 \mathrm{RT}) \mathrm{Eh}\right.$, where $\mathrm{F}$ is the Faraday constant $=96.4935 \times 10^{4} \mathrm{~J} /$ volt equiv].

\section{D.2 NONEQUILIBRIUM CHEMICAL PROCESSES}

Equilibrium thermodynamics is useful to indicate the direction in which an overall reaction should proceed. Most chemical reactions, however, occur in a series of steps, one or more of which may require the input of energy into the system. This results in a series of energetic barriers that needs to be overcome for the reaction to run to completion. The reaction rate is faster for systems that are far out of equilibrium, and it tends to decrease as the equilibrium state is approached. The rate at which equilibrium is approached will depend on a variety of properties. For example, high temperature and pressure, and high surface area all contribute to supply the energy necessary to overcome kinetic barriers and tend to favor faster rates of reaction. Processes such as catalysis and organic reactions serve to lower kinetic barriers speeding up reaction rates. If enough time is available in a closed system with constant temperature and 
pressure conditions, an elementary reaction of the form given by Eq. (D-1) will eventually reach equilibrium, and the development given in Eqs. (D-2) through (D-6) is appropriate. In many systems, however, the time available is insufficient to overcome the kinetic barriers, allowing the reaction to reach a steady-state metastable equilibrium condition. Under these conditions, chemical kinetics are used to examine the rate at which chemical reaction occurs.

An elementary one-step chemical reaction of the form given in Eq. (D-1) is composed of a forward (subscript $f$ ) reaction component (left to right by convention) and a backward (subscript b) reaction component. The net rate of reaction, $\mathrm{r}(\mathrm{moles} / \mathrm{s})$, is the difference between the rate of these two components (i.e., $r=r_{f}-r_{b}$ ). Each direction of the reaction is also described by a rate constant. At equilibrium, the forward and backward rate constants are related to the equilibrium constant such that

$$
\mathrm{K}=\frac{\mathrm{k}_{\mathrm{f}}}{\mathrm{k}_{\mathrm{b}}}
$$

where

$$
\begin{aligned}
& k_{\mathrm{f}}=\text { forward reaction rate constant }\left(\mathrm{s}^{-1}\right) \text {, and } \\
& \mathrm{k}_{\mathrm{b}} \quad=\text { backward reaction rate constant }\left(\mathrm{s}^{-1}\right)
\end{aligned}
$$

Through this relationship, net reaction rate constants are expressed as a function of temperature according to the Arrhenius equation

$$
\mathrm{k}_{\mathrm{r}}=A_{\mathrm{P}} \exp \left(-\frac{\mathrm{E}_{\mathrm{a}}}{\mathrm{R}_{\mathrm{I}} \mathrm{T}}\right)
$$

where

$$
\begin{aligned}
& \mathrm{k}_{\mathrm{d}}=\text { net reaction rate constant }\left(\mathrm{s}^{-1}\right) \\
& \mathrm{A}_{\mathrm{p}}=\text { pre-exponential constant }\left(\mathrm{s}^{-1}\right), \text { and } \\
& \mathrm{E}_{\mathrm{a}}=\text { the activation energy }(\mathrm{J} / \mathrm{mole}) \text { for the reaction. }
\end{aligned}
$$

Reactions can be either reversible with comparable values for both $\mathrm{k}_{\mathrm{f}}$ and $\mathrm{k}_{\mathrm{b}}$ or irreversible if $\mathrm{k}_{\mathrm{b}}$ is small relative to $\mathrm{k}_{\mathrm{f}}$. Generally determined experimentally through a set of time-series experiments, these constants are incorporated into mathematical expressions to define the progress of the reaction according to a rate law. The form that the mathematical expression takes is referred to as the order of the rate law. Defined as the change in concentration of a species $I$, of interest, the rate law takes the general form

$$
\frac{\mathrm{dm}_{\mathrm{I}}}{\mathrm{dt}}=\mathrm{k}_{\mathrm{r}} \mathrm{m}_{\mathrm{I}}^{\mathrm{n}}
$$

where $k_{\mathrm{r}}$ is the net rate constant and $\mathrm{m}_{\mathrm{I}}$ is molality. The order of the reaction with respect to a single reactant (or product) is referred to as the partial order of the reaction and is equal to the exponent $n$ in Eq. (D-9) (e.g., if $n_{B}=2$, the reaction is second-order with respect to $B$ ). The overall order of the reaction is the sum of all of the partial orders (Richardson and McSween, 1989).

The rate of reaction is generally controlled by several steps. These include the transport of reactants to an interface, the chemical reaction of these reactants, and the transport of the products away

$$
\text { D-5 }
$$

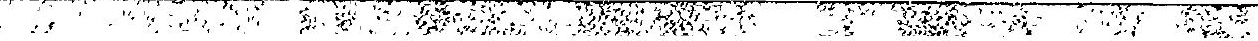


from the interface. The slowest of these steps will become the rate-limiting step for the progress of the chemical reaction. In addition, each one of these steps may be comprised of one or more pathways that lead to either sequential or parallel formation of intermediate compounds, through a series of reactions such as

$$
\mathrm{bB}_{(\mathrm{s})}+\mathrm{cC}_{(\mathrm{aq})} \rightleftharpoons \mathrm{d} \mathrm{D}_{(\mathrm{aq})} \rightleftharpoons \mathrm{e} \mathrm{E}_{(\mathrm{s})}+\mathrm{f} \mathrm{F}_{(\mathrm{aq})}
$$

In this case, $B$ and $C$ react to form products, $E$ and $F$, through the intermediate compound, $D$.

Within the framework of the Arrhenius equation [Eq. (D-8)], reactants must overcome one or more energetic barriers in moving towards equilibrium, passing through a high-energy activated complex. Transition state theory (e.g., Stumm and Morgan, 1981; Lasaga, 1981b) has been developed as a means of understanding the processes involved in the formation of the activated complex at the peak of the energy barrier. This complex is assumed to be in equilibrium with the products such that

$$
a A+b B=X_{A C T}
$$

where $\mathrm{X}_{\mathrm{ACT}}$ represents the activated complex. For the simple case where $\mathrm{a}=\mathrm{b}=1$, the mass action for this reaction simplifies to

$$
\mathrm{K}_{\mathrm{ACT}}=\frac{{ }^{a_{\mathrm{X}}}}{a_{\mathrm{ACT}} a_{\mathrm{B}}}
$$

where $a_{\mathrm{I}}$ represents the activity of the species $\mathrm{I}$. The decomposition of $\mathrm{X}_{\mathrm{ACT}}$ to products follows the general form

$$
\mathrm{X}_{\mathrm{ACT}}=\mathrm{cC}+\mathrm{dD}
$$

As described by transition state theory, the frequency at which $\mathrm{X}_{\mathrm{ACT}}$ decomposes to form products is

$$
v_{A C T}=\frac{k_{B} T}{h_{P}}
$$

where

$$
\begin{aligned}
& \mathrm{v}_{\mathrm{ACT}}=\text { translational frequency }\left(\mathrm{s}^{-1}\right) \\
& \mathrm{k}_{\mathrm{B}}=\text { Boltzmann's constant }\left(1.3805 \times 10^{-23} \mathrm{~J} / \mathrm{K}\right), \\
& \mathrm{h}_{\mathrm{P}}=\text { Planck's constant }\left(6.6256 \times 10^{-34} \mathrm{~J}-\mathrm{s}\right), \text { and } \\
& \mathrm{T} \quad=\text { absolute temperature }(\mathrm{K}) .
\end{aligned}
$$

The rate constant for the net reaction $\left(k_{1}\right)$ can therefore be expressed as

$$
\mathrm{k}_{\mathrm{r}}=\frac{\mathrm{k}_{\mathrm{B}} \mathrm{T}}{\mathrm{h}_{\mathrm{P}}} \mathrm{a}_{\mathrm{x}_{\mathrm{ACT}}}
$$

Combining equations D-6, D-12, and D-15 


$$
\mathrm{k}_{\mathrm{r}}=\frac{\mathrm{k}_{\mathrm{B}} \mathrm{T}}{\mathrm{h}_{\mathrm{P}}} \mathrm{K}_{\mathrm{ACT}}=\left[\exp \left(-\frac{\Delta \mathrm{G}_{\mathrm{ACT}}}{\mathrm{R}_{\mathrm{I}} \mathrm{T}}\right)\right]
$$

where

$$
\left.\Delta \mathrm{G}_{\mathrm{ACT}}=\text { standard Gibbs free energy of activation ( } \mathrm{J} / \mathrm{mole}\right) \text {. }
$$

From Eq. (2-10), the standard free energy of activation is related to the enthalpy $\left(\Delta H_{A C T}\right.$ in $\left.J / m o l e\right)$ and entropy $\left(\Delta S_{A C T}\right.$ in $\mathrm{J} /$ mole- $\left.K\right)$ of activation such that

$$
\Delta \mathrm{G}_{\mathrm{ACT}}=\Delta \mathrm{H}_{\mathrm{ACT}}-\mathrm{T} \Delta \mathrm{S}_{\mathrm{ACT}}
$$

and Eq. (D-16) becomes

$$
\mathrm{k}_{\mathrm{r}}=\frac{\mathrm{k}_{\mathrm{B}} \mathrm{T}}{\mathrm{h}_{\mathrm{P}}}\left[\exp \left(\frac{\Delta \mathrm{S}_{\mathrm{ACT}}}{\mathrm{R}_{\mathrm{I}}}\right)\right]\left[\exp \left(\frac{-\Delta \mathrm{H}_{\mathrm{ACT}}}{\mathrm{R}_{\mathrm{I}} \mathrm{T}}\right)\right]
$$

Since $\Delta \mathrm{H}_{\mathrm{ACT}}=\mathrm{E}_{\mathrm{a}}-\mathrm{R}_{\mathrm{I}} \mathrm{T}$ (Stumm and Morgan, 1981; Lasaga, 1981b), Eq. (D-17) can be rewritten

$$
k_{r}=e\left[\frac{k_{B} T}{h_{P}}\right]\left[\exp \left(\frac{\Delta S_{A C T}}{R_{I}}\right)\right]\left[\exp \left(-\frac{E_{a}}{R_{I} T}\right)\right]
$$

From comparison with Eq. (D-8), it can be seen that the pre-exponential factor from the Arrhenius equation $\left(A_{p}\right)$ is

$$
A_{P}=e\left[\frac{k_{B} T}{h_{P}}\right]\left[\exp \left(\frac{\Delta S_{A C T}}{R_{I}}\right)\right]
$$

which illustrates the dependence of the pre-exponential factor on temperature and the entropy of activation.

\section{D.3 REACTION PROGRESS MODELS}

One critical aspect of a chemical system is the transfer of mass between the solid, gas, and liquid phases during chemical reactions. Using the principles outlined above, an approach has been developed (e.g., Helgeson, 1970) to model reaction progress in a natural system.

Reaction progress models describing systems involving interaction between an aqueous solution and minerals are a way of monitoring the approach to equilibrium through a series of sequential homogeneous equilibrium reactions. Helgeson (1979) notes that the assumption of partial equilibrium accepts that, although the system under consideration is likely to be out of equilibrium with respect to one or more processes, it can also be considered to exist in equilibrium with respect to at least one process. This is considered valid in mineral-water systems where homogeneous reaction between species in the aqueous phase generally occur orders of magnitude more rapidly than heterogeneous reactions between minerals and solution. In a similar fashion, an assumption of local equilibrium within the aqueous phase assumes that, at small scales of time and space, chemical reactions maintain homogeneous and 
heterogeneous equilibrium and the degree of supersation required to induce precipitation of minerals is negligible compared to total mass transfer between phases for the system.

In a given irreversible chemical process involving $\mathrm{k}$ simultaneous reactions, the chemical affinity for the jth elementary reaction $\left(A_{j}{ }^{*}\right.$ in $\mathrm{J} /$ mole) has been defined (De Donder and van Rysselberghe, 1936; Prigogine, 1955; Helgeson, 1979) such that

$$
A_{j}^{*}=R_{I} T \ln \left[K_{j} / Q_{j}\right]=-\left(\frac{\partial G_{j}}{\partial \xi_{j}}\right)_{P, T}
$$

where $R_{\mathrm{I}}, T, \mathrm{G}_{\mathrm{j}}, \mathrm{K}_{\mathrm{j}}$, and $\mathrm{Q}_{\mathrm{j}}$ are as defined above. The reaction progress variable $\xi_{\mathrm{j}}$ (moles) is a measure of the degree to which the jth elementary reaction has proceeded towards equilibrium. $\xi_{\mathrm{j}}$ represents the number of moles of a given reactant converted into products by the jth reaction, divided by the stoichiometric coefficient of the reactant in the $j$ th reaction. At equilibrium, the term $K_{j} / Q_{j}=1$ and $A_{j}^{*}=0$ from Eq. (D-21), and the jth reaction becomes reversible. Overall equilibrium is only achieved when $A_{k}{ }^{*}=0$ for all $k$ reactions involved in the process.

For the set of simultaneous reactions describing an overall irreversible geochemical process involving a given set of minerals, aqueous species, and/or gases, the slowest (rate limiting) reaction can be treated in the same manner discussed for Eq. (D-21). This leads to the development of an overall reaction progress variable $(\xi)$ for this reaction (Helgeson, 1979). Rates for other reactions in the process involving minerals and solution can be expressed in terms of $\xi$, and the reversible mass transfer for the equilibrium subsystem imposed by an assumption of partial and local equilibrium can be expressed as a function of $\xi$. As the rate limiting reaction advances towards equilibrium, the system changes as a function of overall reaction progress. The subsystem of equilibrium reactions (homogeneous and heterogeneous) are assumed to change instantaneously to maintain equilibrium. By proceeding through a series of closely spaced partial equilibrium states, a reaction path is therefore defined as a function of overall reaction progress. At each step as the equilibrium subsystem proceeds along the reaction path, mass action and mass balance are used to calculate mass distribution for the equilibrium subsystem. Mass transfer between the solution and the minerals, governed by the assumption of local equilibrium, is monitored. The saturation of the aqueous phase is evaluated with respect to possible minerals. If the solution is supersaturated (i.e., $Q_{j}>K_{j}$ ) with respect to a mineral not already present, it is added to the equilibrium system. An equilibrium mass action expression for the heterogeneous reaction is added to the governing equations describing the equilibrium subsystem and the mass balance is modified to take into account the presence of a new mineral; the distribution of aqueous species is recalculated using the new set of mass balance/mass action expressions, and some mass of the new mineral in local equilibrium with the solution is added to the solid phase. If the solution is undersaturated with respect to a mineral that is already present, some mass (calculated by mass action/mass balance) is dissolved into solution until either the solution becomes saturated or until it is eventually removed from the system, at which point it is removed from the mass action and mass balance equations. As long as a mineral is present, it is assumed to remain in local heterogeneous equilibrium with the solution. The reaction progress is incremented by a small, finite amount $(\Delta \xi)$, and the process is repeated for the next step along the reaction path.

Absolute reaction rates are not necessary to model a reaction path as a function of overall reaction progress $(\xi)$. However, if the rate laws and rate constants governing the heterogeneous reactions 
are known, reaction progress can be calculated as a function of time (e.g., Helgeson and Murphy, 1983) such that

$$
r=\frac{\partial \xi}{\partial t}=\frac{k_{f} A^{*}}{R_{I} T}
$$

For a reaction progress step, mass is transferred between the solid and aqueous phase according to its absolute rate; the total time involved in the reaction progress step is calculated by integrating the rate law over the amount of mass transferred during the step.

\section{D.4 THERMODYNAMIC DATABASES}

A great deal of effort has been made to determine experimentally the thermodynamic properties necessary for the modeling of chemical processes (e.g., Helgeson et al., 1978; Parkhurst et al., 1980; Nordstrom et al., 1990; Wolery et al., 1990). There have been many compilations of data that vary in the amount of attention paid to the quality and internal consistency of the data, completeness of the dataset, referencing data sources and the pressure and temperature ranges over which the data are valid. Several recent studies (Baes and Mesmer, 1976; Helgeson et al., 1978; Krupka et al., 1988; Phillips et al., 1988; Serne et al., 1990) have outlined strategies and selection criteria for evaluating chemical data for inclusion in a comprehensive database. There are also approaches for estimating thermodynamic data for some less well characterized compounds (Phillips et al., 1988). With respect to more common elements and phases, abundant experimental data are available. These data have been refined through application in a wide variety of chemical environments, and the agreement between different data sources is often good over a broad range of temperature and pressure. However, for many trace elements, such as the chemically complex transuranic elements important in HLW disposal, available data are sparse, and agreement is frequently poor. To address these uncertainties, the Nuclear Energy Agency (NEA) has undertaken to develop comprehensive, consistent databases for critical radionuclides (e.g., Grenthe et al., 1992).

Table D-1 (Serne et al., 1990) is a listing of some of the more commonly used compilations of thermodynamic data. Special emphasis is placed on those data related to geochemical reaction codes. The reader is referred to the original reference (and the references therein) for specifics on how the data were generated and selected.

\section{D.5 COUPLING WITH OTHER PROCESSES}

Through changes in fluid, gas, and solid properties, thermal, mechanical, and hydrological processes in the repository environment will be affected through coupling with chemical reactions of the forms given in Eqs. (D-1) and (D-10). These effects have been discussed in more detail in Section 2. In a general sense, however, these changes may include the following:

- Fluids - The presence of aqueous species in solution can affect a variety of fluid properties, including density, viscosity, vapor pressure (boiling curve), heat capacity, and the degree of şaturation with respect to different solid phases. In addition, molecular diffusion along chemical gradients may lead to solute transport in the absence of advection. Similarly, diffusion of uncharged water molecules across semipermeable clay layers in response to salinity gradients can result in an osmotic pressure gradient which, in turn, can affect fluid flow. 
- Solids - The presence or absence of solid phases is determined by the solubility of the mineral of interest and the kinetics which control precipitation and dissolution. Hydrologic properties of the medium are affected since reaction progress can alter porosity and permeability. In addition, the production of intermediate species through incongruent mineral dissolution can produce significant changes in properties, such as volume and sorptive capacity, depending on the phase produced. Mechanical properties of a medium can also be affected by chemical processes, such as oxidation or aqueous corrosion of metals, or weakening of natural materials through dissolution and weathering. Through reaction, solid phases can act as a buffer on the composition of fluids (e.g., pH, Eh, and ionic strength). Electrolytes can also be removed (or added) from solution either by the precipitation/dissolution of stoichiometric compounds or by the inclusion of electrolytes as trace impurities in other solid phases. In addition to precipitation/dissolution, solids can also act as a source or a sink through a variety of surface reactions, such as ion exchange, adsorption/desorption, and matrix diffusion.

- Gases - Each gas phase present in a system will move towards chemical equilibrium with the liquid phase through the exchange of molecules across the gas/water interface. Changes in gas partial pressures will set up pressure gradients and lead to a new set of equilibrium conditions if time is sufficient. Gas is produced through biological or chemical reactions and can lead to increased pressures and buffering of solution $\mathrm{pH}$, Eh, and composition. Since several radionuclides are thought to occur as gas phases $\left({ }^{14} \mathrm{C},{ }^{129} \mathrm{I}\right)$, an increased gas flux could lead to increased radionuclide migration to the accessible environment.

Equations (D-6) and (D-22) demonstrate some of the temperature dependence of equilibrium chemistry and reaction rates. In addition, several chemical parameters (log $\mathrm{K}$, rate constants, $\mathrm{pH}$, activity/concentration relationships) vary as a function of temperature. In a natural system, hydrologic conditions can also have a significant effect on system chemistry. Fluid flow will determine, to a large extent, whether or not the system is open or closed to different given solid, aqueous, or gas phases. Flow rates will determine the time available for a reaction to approach equilibrium. Fluid flow paths determine fluid compositions and which minerals are involved in chemical reactions.

Based on analyses of the anticipated thermal loading at Yucca Mountain (Pruess and Wang, 1987), temperature is likely to be one of the primary couples that affects system chemistry. Fluid flow and saturation (e.g., Verma and Pruess, 1988; Tumer, 1991a,b; Lichtner, 1992) are also critical in applying chemical principles to modeling. the transport of radionuclides from the repository to the accessible environment. Clearly, chemistry is closely coupled with the temperature and fluid flow regimes of the repository environment. Understanding the complex feedbacks between these regimes is an important step in evaluating the ability of the repository to isolate successfully high-level radioactive waste. Mechanical effects are likely to be less direct, involving aspects such as pulse inputs due to barrier failure, or providing access for fluids and gases to the waste package. 
Table D-1. Examples of tabulations of thermodynamic data (modified from Serne et al., 1990).

\begin{tabular}{|c|c|}
\hline Title & Reference \\
\hline The Hydrolysis of Cations & Baes and Mesmer (1976) \\
\hline $\begin{array}{l}\text { JANAF Thermochemical Tables. 3rd Edition. Part I (Al-Co); } \\
\text { Part II }(\mathrm{Cr}-\mathrm{Zr})\end{array}$ & Chase et al. (1985a,b) \\
\hline CODATA: Key Values for Thermodynamics & Cox et al. (1989) \\
\hline $\begin{array}{l}\text { Summary and Critique of the Thermodynamic Properties of } \\
\text { Rock-Forming Minerals }\end{array}$ & Helgeson et al. (1978) \\
\hline Standard Potentials in Aqueous Solutions & Bard et al. (1985) \\
\hline Handbook of Thermodynamic Data & Naumov et al. (1974) \\
\hline $\begin{array}{l}\text { The Chemical Thermodynamics of Actinide Elements and } \\
\text { Their Compounds. Part } 1 \text { (The Actinide Elements); Part } 2 \\
\text { (The Actinide Aqueous Ions); Part } 3 \text { (Miscellaneous Actinide } \\
\text { Compounds), and others }\end{array}$ & $\begin{array}{l}\text { Oetting et al. (1976) } \\
\text { Fuger and Oetting (1976) } \\
\text { Cordfunke and O'Hare (1978) }\end{array}$ \\
\hline $\begin{array}{l}\text { Thermodynamic Tables for Nuclear Waste Isolation: } \\
\text { Aqueous Solutions Database }\end{array}$ & Phillips et al. (1988) \\
\hline Chemical Thermodynamics of Technetium & Rard (1989) \\
\hline $\begin{array}{l}\text { Thermodynamic Properties of Minerals and Related } \\
\text { Substances at } 298.15 \mathrm{~K} \text { and } 1 \mathrm{Bar}\left(10^{5} \text { Pascals) Pressure and }\right. \\
\text { at Higher Temperatures }\end{array}$ & Robie et al. (1978) \\
\hline $\begin{array}{l}\text { Selection of Standard Free Energies of Formation for Use in } \\
\text { Soil Chemistry }\end{array}$ & $\begin{array}{l}\text { Sadiq and Lindsay }(1979,1981) \\
\text { Elrashidi and Lindsay }(1984)\end{array}$ \\
\hline Critical Stability Constants. Volume 4: Inorganic Complexes & Smith and Martell (1976) \\
\hline $\begin{array}{l}\text { The NBS Tables of Chemical Thermodynamic Properties. } \\
\text { Selected Values for Inorganic and } C_{1} \text { and } C_{2} \text { Organic } \\
\text { Substances in SI Units }\end{array}$ & Wagman et al. (1982) \\
\hline NEA-TDB: Chemical Thermodynamics of Uranium & Grenthe et al. (1992) \\
\hline $\begin{array}{l}\text { A Tabulation of Thermodynamic. Data for Reactions } \\
\text { Involving } 58 \text { Elements Common to Radioactive Waste } \\
\text { Package Systems }\end{array}$ & Benson and Teague (1980) \\
\hline $\begin{array}{l}\text { Revised Chemical Equilibrium for Major Water-Mineral } \\
\text { Reactions and Their Limitations }\end{array}$ & Nordstrom et al. (1990) \\
\hline The CHEMVAL Database & Read and Broyd (1991) \\
\hline $\begin{array}{l}\text { SUPCRT92: A Software Package for Calculating the } \\
\text { Standard Molal Thermodynamic Properties of Minerals, } \\
\text { Gases, Aqueous Species, and Reactions from } 1 \text { to } 5000 \text { bars } \\
\text { and } 0 \text { to } 1000^{\circ} \mathrm{C} \text {. }\end{array}$ & $\begin{array}{l}\text { Johnson et al. (1991) } \\
\text { Tanger and Helgeson }(1988) \\
\text { Shock and Helgeson }(1988,1990)\end{array}$ \\
\hline $\begin{array}{l}\text { Thermodynamic Values at Low Temperature for Natural } \\
\text { Inorganic Materials: An Uncritical Summary }\end{array}$ & Woods and Garrels (1987) \\
\hline
\end{tabular}


A Literature Review of Coupled Thermal-Hydrologic-Mechanical-

Chemical Processes Pertinent to the Proposed High-Level Waste Repository at Yucca Mountain

3. OATE REPORT PUBLISHED \begin{tabular}{l|l} 
MONTH & YEAR \\
July & 1993
\end{tabular}

4. FIN OR GRANT NUMBER D1035

6. TYPE OF REPORT Technical

Center for Nuclear Waste Regulatory Analyses

Southwest Research Institute

6220 Culebra Road

San Antonio, TX 78228-0510

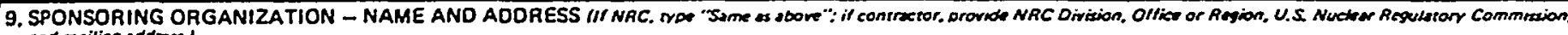
and meiling sodornes!

Division of High-Level Waste Management

Office of Nuclear Material Safety and Safeguards

U.S. Nuclear Regulatory Commission

Washington, DC 20555

10. SUPPLEMENTARY NOTES

\section{ABSTRACT 1200 mordor ora!}

A literature review has been conducted to determine the state of knowledge available in the modeling of coupled thermial $(T)$, hydrologic $(H)$, mechanical $(M)$, and chemical $(C)$ processes relevant to the design and/or performance of the proposed high-level waste (HLW) repository at Yucca Mountain, Nevada. The review focuses on identifying coupling mechanisms between individual processes and assessing their importance (i.e., if the coupling is either important, potentially important, or negligible). The significance of considering THMC-coupled processes lies in whether or not the processes impact the design and/or performance objectives of the repository.

A review, such as reported here, is useful in identifying which coupled effects will be important, hence which coupled effects will need to be investigated by the U.S. Nuclear Regulatory Commission in order to assess the assumptions, data, analyses, and conclusions in the design and performance assessment of a geologic repository. Although this work stems from regulatory interest in the design of the geologic repository, it should be emphasized that the repository design implicitly considers all of the repository performance objectives, including those associated with the time after permanent closure. The scope of this review is considered beyond previous assessments in that it attempts (with the current stateof-knowledge) to determine which couplings are important, and identify which computer codes are currently available to model coupled processes.

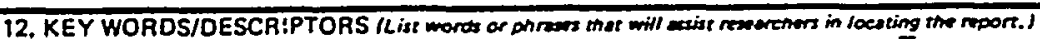

Coupled processes

Thermal-Hydrologic-Mechanical-Chemical Processes

Coupled-effects

THMC processes

Thermal loads

Thermomechanical

Thermohydrologic

Thermochemical

Geochemical

One-way coupling
Two-way coupling

Three-way coupling

Four-way coupling
13. AVAILABILITY STATEMENT

Unt imited

14. SECURITY CLASSIFICATION

(ThAs Pepl

Unclassified

This Reoor?

Unclassified

15. NUMBER OF PAGES 